\title{
THE AGING HIPPOCAMPUS: A MULTILEVEL ANALYSIS IN THE RAT
}

IRA DRISCOLL

M.S., The University of New Mexico, 2002

B.A., The University of New Mexico, 2000

\author{
A Thesis \\ Submitted to the School of Graduate Studies \\ of the University of Lethbridge \\ in Partial Fulfilment of the \\ Requirements for the Degree \\ PH.D., NEUROSCIENCE \\ Canadian Centre for Behavioural Neuroscience, Department of Neuroscience \\ University of Lethbridge \\ LETHBRIDGE, ALBERTA, CANADA
}

(c) Ira Driscoll, 2005 


\begin{abstract}
The purpose of the current thesis was twofold: (1) to examine various factors that might be contributing to age-related learning and memory deficits specifically related to the hippocampus, and (2) to validate our rat model of aging, employing a multilevel analysis. We found age-related deficits on both spatial and non-spatial hippocampus-dependent tasks that were accompanied by structural alterations observed both in vivo (volume, but not neuronal metabolic function) and post mortem (neuronal density and neurogenesis, but not synaptic or mitochondrial density). Furthermore, our results suggest that the observed hippocampal structural changes, namely decreased volume and neurogenesis, predict learning and memory deficits, and both can be accounted for by neurogenic reduction. In addition, the above-mentioned pattern of age-related deficits closely resembles that seen in humans, suggesting the present rat version of aging to be a very useful model for investigating hippocampal aging in humans.
\end{abstract}




\section{Acknowledgements}

First and foremost, I would like to thank my advisor, Dr. Robert Sutherland, for this amazing opportunity. I feel very lucky to have been given a chance and am grateful for your invaluable guidance, mentorship, and support. I would also like to thank my committee members, Dr. William Brooks, Dr. Bryan Kolb, Dr. Ian Whishaw, and Dr. Olga Kovalchuk, for their valuable recommendations and support pertaining to this thesis. Special thanks to Ian Whishaw, for going over and above his call of duty and providing precious advice and learning opportunity. One should be so lucky to always have someone like you around, Ian. Dr. Kolb, for the opportunity to learn and conduct some research in your laboratory. Also, my sincere gratitude to Dr. Brooks for his continuous support and consultation, and for coming to Lethbridge multiple times on my behalf.

I would like to acknowledge the Calgary group from the Experimental Imaging Centre - Dr. Boguslaw Tomanek, Tadeus Foniok, and Dave Kirk. It was a pleasure working with all of you. Special thanks to Dave for providing the entertainment during scanning and to Tad for putting up with it. My deepest gratitude to Helen Petropoulos and Laura Rowland for providing a spectroscopy hotline, and for the friendship and support that you've shown to me throughout the years. 
Many, many thanks to Marie-Helene Monfils. A mere word of thanks is not enough for all that you have done, nor is a page to list all that I'm thankful for. Marie, I will always be happy to look through peepholes for you. Sarah Howard and Jared Stone, I feel privileged to have worked with you. Your hard work and friendship are greatly appreciated. Kristin Johnson, for sharing her Ki67 procedure with me. Doug Bray, for teaching me how to use an Electron Microscope and all that is associated with it, but also for having a seemingly inexhaustible amount of patience.

Very special thanks to Glen Prusky for the opportunity to collaborate and to toboggan in Mountain View, and to the members of his laboratory for accepting me as one of their own, especially Trevor McGill and Byron Silver. Rob McDonald and his laboratory for collaborations, especially Nhung Hong it's been a pleasure working with you.

Special thanks to Janice Sutherland for many years of support and friendship. Naomi Cramer, for being so kind and helpful from the very first moment I met you. Afra Foroud, for being a dear friend and talking me into dancing again. Christine Reinhart, for making sure I wasn't the only quirky one around - that's what friends are for. Penny Vandenberg, for making my life so much easier. Daniel Poda and Dawn Danka, for their help and friendship.

Many thanks to various people at the CCBN whose kindness is greatly appreciated. And to those not so kind - it just goes to show that what 
doesn't kill you, certainly does make you stronger, so I suppose thanks are in order.

And last, but not least, to my family. To my husband and my best friend, Jason, thank you for the many years of support and for always believing in me, but most of all for the immense amount of patience, understanding, and sacrifice on your part. To my parents, who sacrificed everything they had so that I could have opportunities. I hope I made you proud. To my sister, Marijam, who provided immeasurable support and entertainment over the years. And finally to the rest of my family and all of my friends, your encouragement is greatly appreciated. 


\section{Table of Contents}

1. Introduction 1

2. The Hippocampal Formation 3

3. The Function of the Hippocampus 5

3.1 Rationale for Memory Tests of Hippocampal Function 8

3.2 Hippocampus-dependent Learning and Memory in Rodents

3.2.1 Spatial Navigation

3.2.2 Transverse Patterning Discrimination 18

3.3 Hippocampus-dependent Learning and Memory in Humans

3.3.1 Virtual Navigation $\quad 20$

3.3.2 Transverse Patterning Discriminations $\quad 26$

4. The Aging Hippocampus 28

$\begin{array}{ll}4.1 \text { Physiological Changes } & 28 \\ 4.2 \text { Structural Changes } & 31 \\ 4.3 \text { Biochemical Changes } & 34 \\ 4.4 \text { Neurogenesis } & 39\end{array}$

5. Human Model of Rat Aging 46

6. Experiment 1 - Visual Acuity 53

$\begin{array}{ll}6.1 \text { Background } & 53 \\ 6.2 \text { Materials and Methods } & 55 \\ 6.3 \text { Results } & 61\end{array}$

6.4 Discussion 62

7. Experiment 2 - Long -Term Potentiation 64

7.1 Background 64

7.2 Materials and Methods $\quad 67$

$\begin{array}{ll}7.3 \text { Results } & 70\end{array}$

7.4 Discussion $\quad 73$

8. Experiment 3 - Spatial Learning and Memory 78

8.1 Background 78

8.2 Materials and Methods $\quad 80$

8.3 Results $\quad 82$

8.4 Discussion 85

9. Experiment 4 - The Transverse Patterning Problem 88

9.1 Background 88

9.2 Materials and Methods 91 
9.3 Results $\quad 96$

9.4 Discussion 98

10. Experiment 5 - Non-spatial Learning and Memory 104

10.1 Background 104

10.2 Materials and Methods $\quad 105$

10.3 Results 108

10.4 Discussion 110

11. Experiment 6 - Magnetic Resonance Imaging and Spectroscopy 113

11.1 Background 113

11.2 Materials and Methods $\quad 116$

11.3 Results 120

11.4 Discussion 121

12. Experiment 7 - Light and Electron Microscopy 124

12.1 Background 124

12.2 Materials and Methods $\quad 126$

12.3 Results 132

12.4 Conclusion 134

13. Experiment 8 - Neurogenesis 137

13.1 Background 137

13.2 Materials and Methods $\quad 139$

13.3 Results 143

13.4 Discussion 145

14. Correlations 149

$\begin{array}{ll}14.1 \text { Partial Correlations } & 155\end{array}$

15. General Discussion 158

16. Closing Comments 168

17. Conclusion 170

18. References 173 


\section{List of Tables}

1. Discrimination training procedures.

2. Partial correlations. 


\section{List of Figures}

1. The Visual Water Task. 195

2. Visual acuity - Static gratings. 196

3. (A and B) Visual acuity - OptoMotry. 197

4. Electrophysiology - Electrode placements. 198

5. Effects of HFS sessions on the evoked potentials. 199

6. Representative field potentials. 200

7. Morris water task performance - hidden platform trials. 201

8. Morris water task performance - 24-hour retention. 202

9. Morris water task performance - visible platform trials. 203

10. The Visual Discriminanda. 204

11. A representative NMDA damage. 205

12. Elemental discrimination performance - Lesions. 206

13. Learning rate - Elemental discriminations. 207

14. Transverse patterning discriminations - Lesions. 208

15. Elemental discriminations - Aging. 209

16. Transverse patterning discriminations - Aging. $\quad 210$

$\begin{array}{ll}\text { 17. A representative MR image of the rat hippocampus. } & 211 \\ \text { 18. (A and B) Magnetic Resonance Spectroscopy (MRS) acquisition. } & 212\end{array}$

$\begin{array}{ll}\text { 18. (A and B) Magnetic Resonance Spectroscopy (MRS) acquisition. } & 212 \\ \text { 18. (C) A representative raw MRS spectrum. } & 213\end{array}$

19. Age-related differences in intracranial volume. 214

20. Age-related differences in absolute hippocampal volume. $\quad 215$

21. Age-related differences in normalized hippocampal volume. $\quad 216$

22. Semi-thin $(1 \mu \mathrm{m})$ section selection for light microscopy. 217

23. Electron micrograph depicting a larger area containing synapses. $\quad 218$

24. Electron micrograph depicting a larger area containing mitochondria. $\quad 219$

25. Changes in neuronal density with age. 220

26. A confocal image of BrdU/NeuN double-labeled cells. 221

27. Representative Doublecortin labeling. 222

28. Representative Ki67 labeling. 223

29. The number of BrdU labeled cells. 224

30. The number of BrdU/NeuN double-labeled cells. 225

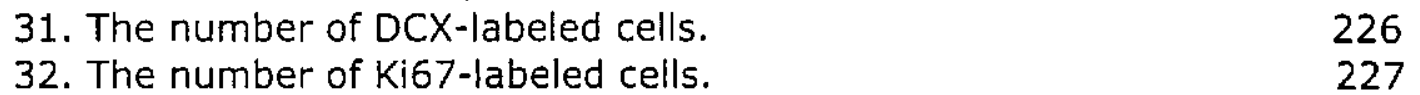

$\begin{array}{ll}\text { 32. The number of Ki67-labeled cells. } & 227 \\ 33 . \text { Correlations between MWT and hippocampal volume. } & 228\end{array}$

34. Correlations between TPDT and hippocampal volume. 229

35. Correlations between MWT and DCX-labeled cells. 230

36. Correlations between TPDT and DCX-labeled cells. 231

37. Correlations between hippocampal volume and DCX-labeled cells. 232

38. Correlations between BrdU/NeuN- and DCX-labeled cells. 233

39. Correlations between Ki67-labeled cells and TPDT, 234

40. Correlations between Ki67-labeled cells and hippocampal volume. 235

41. Correlations between Ki67- and DCX-labeled cells. 236 


\section{Introduction}

When I was younger, I could remember anything, whether it had happened or not; but my faculties are decaying now and soon I shall be so I cannot remember any but the things that never happened. It is sad to go to pieces like this but we all have to do it. Mark Twain

The study of aging attempts to understand the changing capacities of the elderly as a normal developmental process and the understanding of the neurobiology of aging is an integral part of that framework. Developmental boundaries and failure to manifest certain changes in the usual course of development are easily recognized early in life, such as failure to walk or talk by certain age. On the other end of the developmental spectrum the boundaries of normal aging as distinct from pathology, however, are not clearly defined. Age-related changes do not seem to differ from manifestations of age-related pathology, at least not in the early stages. Both normal and pathological aging are characterized by learning and memory impairments, and there is no consensus on the precise nature of underlying neurobiological changes.

Alzheimer's disease, on the other hand, is a debilitating neurological disorder of old age that is characterized by a progressive loss of cognitive function, memory loss being a hallmark. $A D$ has damaging effects on the quality and enjoyment of life of the elderly people, both the ones suffering from the disease and those 
caring for them. Currently, efforts are under way to develop treatments for AD, even though we do not fully understand what causes the disease. As critical as it is to find the cure for this devastating condition, a good understanding of boundaries between normal and pathology is necessary.

Various cognitive domains, subserved by many different brain regions, are affected by aging. Here we restrict our considerations to the tasks that are dependent on the integrity of the hippocampal formation. This focus is based on the fact that age-related cognitive changes, normal and pathological, are intimately linked to the hippocampal formation. The hippocampus has maintained a central role in the realm of learning and memory research over the past 40 years, particularly in memory for cue conjunctions, best exemplified in memory for contexts or spatial locations.

One of the broad aims here is to contribute to the understanding of hippocampus-dependent learning and memory by studying age-related changes in both behavior and in underlying neurobiology. First, the current state of the literature in relation to hippocampal function will be briefly reviewed. Then, the functional deficits will be described and appropriate parallels drawn between the human and rat behavioral literature. Further, magnetic resonance imaging and magnetic resonance spectroscopy findings will be considered to draw plausible links with hippocampus-dependent cognitive impairments that have been observed in both aged humans and rodents. Finally, the current findings will be c onsidered and to conclude, a prospective view will be offered. 


\section{The Hippocampal Formation}

The hippocampus, a medial temporal lobe structure, is a part of the cerebral cortex and the limbic system. Even though the hippocampus is not a part of the superficial cortex, it is not a "subcortical" structure. The hippocampus is bilaterally symmetrical and it is comprised of the hippocampus proper (principally CA1 and CA3) and the dentate gyrus (fascia dentata). Each field contains a densely packed sheet of cells, both pyramidal and granular, which are the principal cell types found in this structure. In addition, each field contains a variety of interneurons that are intermixed with the principal cell types. The hippocampus proper contains CA1, CA2, CA3, and CA4 fields and is primarily comprised of pyramidal cells. CA2 and CA4 fields are very small and not well studied. In contrast, CA1 and CA3 fields have been more extensively explored. CA1 cells enter the alveus and further project to the subiculum, the lateral septal nuclei, and the prefrontal cortex. The CA3 pyramidal cells project primarily to the CA1 region via the Schaffer collaterals, to the lateral septal nuclei via fornix, and have recurrent excitatory connections. The dentate gyrus, the principal recipient of projections from the entorhinal cortex, is primarily comprised of granule cells. It is commonly divided into an upper and a lower blade, between which reside the large polymorph cells in the hilus of the dentate gyrus. The hilar cells play an interesting role in providing efferents that form the part of the hippocampal commissural and ipsilateral association system, the role of which is to provide interhemispheric and intrahemispheric associative connections between components of the hippocampus. Axons from the dentate 
gyrus make connections with dendrites of CA3 pyramidal cells via the mossy fibers. Input and output from the neocortex happens via the entorhinal cortex, which projects to the dentate gyrus, and $\mathrm{CA} 1$ and $\mathrm{CA} 3$ fields via the perforant path. The CA1 field has projections back to the entorhinal cortex via subiculum. For an extensive review of hippocampal anatomy we refer the reader to articles by Amaral \& Witter (1989, 1995). 


\section{The Function of the Hippocampus}

There is now agreement that the hippocampus, although it may have multiple functions, is critically involved in learning and memory. On the other hand, no general consensus has emerged regarding the characteristics of memory processes that are dependent on the hippocampus. According to Nadel and Moscovitch (2001), three fundamental questions emerge in relation to the involvement of this structure in learning and memory. One question is whether all types of learning and memory are affected by hippocampal damage. The second question is linked to hippocampus-related amnesia, specifically whether amnesia is best characterized as a deficit in memory storage or retrieval. And the third question asks whether the hippocampus plays an equivalent role in remote or recent memories. To date, only the first of these three questions seems to be adequately resolved.

Research on memory has traditionally focused on trying to characterize what amnesic patients can or cannot do. Nearly all investigators now agree that most insults to the hippocampal formation alter its function, whether permanently or temporarily, and as a consequence certain information, whether learned before or after the insult, is disrupted. Most of the early studies on hippocampal function sought to identify tasks that are either profoundly impaired or else completely spared following hippocampal damage. In considering the function of the hippocampus and the changes in hippocampus-dependent cognition as we age, it is necessary to consider it from a historical perspective first. 
The pioneering reports on amnesic patient H.M. (Scoville and Miller, 1957), and similar subsequent patients with medial temporal lobe damage that included the hippocampus, revealed that short-term, procedural, and implicit memory are apparently spared even though a significant portion of the hippocampus and sometimes surrounding structures were missing. Cumulatively, assessments of medial temporal lobe damaged patients revealed that not all types of learning and memory are affected by hippocampal damage. Initially it was reported that the memory deficits were limited to the information from experiences within a few years prior to the damage while older memories remained spared.

In particular, for memories acquired before the hippocampus was damaged, more recent memories were lost (retrograde amnesia) but more remote memories appeared to be spared. This kind of dissociation between recent and remote memories (temporally-graded retrograde amnesia) is consistent with the idea that neither retrieval nor very long-term memory storage require intact hippocampal circuitry, and further, that only for recently acquired memories hippocampus plays an essential role in storage and/or retrieval (Corkin, 1984; Milner, 1966). The claims about the temporally-graded processes in retrograde amnesia came under increasingly critical scrutiny in recent years, partly due to new information gained from additional memory testing in medial temporal lobe damaged patients, and partly as a consequence of the emerging evidence from functional imaging. 
Several studies employing fMRI have measured hippocampal activation while participants were remembering recently acquired vs. remotely acquired information. The simplest prediction, considering the results showing temporally graded retrograde amnesia, is that there should be relatively less hippocampal activation as more remote memories are retrieved. In contrast to this prediction, studies found that the hippocampus is activated to the same extent during retrieval of recent and remote memories (Maguire, 1999; Ryan et al., 2001). Also, the period of retrograde amnesia in patients with damage restricted to the hippocampal formation is now known to extend for decades (Cipolloti et al., 2001).

The non-human animal experimental literature is no less controversial with retrograde gradients reported to last a few days to months to no retention of remote information after large hippocampal lesions (Anagnostaras et al., 1999; Bohbot et al., 1998, Clark et al., in press, 2002; Sutherland et al., 2001; Winocur, 1990; Winocur et al., 2001). The debate about differential involvement of the hippocampus in recent vs. remote memories will continue for some time to come before a consensus is achieved. Future research will likely center on two issues: the extent of the lesion and the types of memory tests employed. Also, neuropsychological studies of human memory impairments, which are generally limited to opportunistic encounters with patients, usually involve limited, but rarely precise or complete damage to the hippocampus. The precise experimental controls, in order to understand memory function in detail, can only be imposed through research on non-human animals. A major impediment 
to non-human animal research, however, has been the assessment of declarative or episodic memory.

Episodic memory (Tulving, 1986) is thought to be a characteristic unique to humans. Still, recent studies of memory for cache sites in food storing jays (Griffiths and Clayton, 2001) indicate that several features of episodic memory are present, suggesting that the essential properties may not be as unique to humans as some may think. Such research highlights the need for development of appropriate animal models in order to bridge the gap between human and non-human animal memory research. Therefore, the hippocampus-dependent tasks and theories of hippocampal function that emphasize cross-species comparisons are essential. To that end, the current state of the literature in relation to hippocampal function is briefly reviewed.

\subsection{Rationale for Memory Tests of Hippocampal Function}

There are many theories of hippocampal function. In fact, for the person outside the area, the variety is bewildering. Despite the variety, most modern theories agree that the hippocampus is essential for learning of certain kinds of information and that other systems outside the hippocampus are able to acquire other types of information independently. One common theme found in these theories is that the hippocampus creates a complex representation of an event or an episode such that conjunctions of co-occuring or related cues are bound 
together into a unitary representation. For certain memories the hippocampus plays an essential role in storage and retrieval for at least months and perhaps for decades after the occurrence of the learning episode. The hippocampus is thought to play a central role in memories for facts and for personal events, and is considered to support conscious recollections of experiences. Many fundamental aspects of hippocampal functioning remain a matter of debate and so it would be a mistake to suggest that there is a satisfying consensus. In order to get a feel for the current state of literature on hippocampal function, some of the more influential theories of hippocampal function are reviewed below.

A commonly held view is that the hippocampus plays a fundamental role in a formation of unified representations of an event by integrating multiple cues related to that event (Eichenbaum, 2001; Gaffan, 1994; O'Reilly and Rudy, 2001; Rudy and Sutherland, 1995; Squire, 1992; Sutherland and Rudy, 1989; Wickelgren, 1979). Possibly the most influential theory of hippocampal function to emerge in the past three decades is the cognitive mapping theory of O'Keefe \& Nadel (1978)

The principal driving force behind the development of cognitive mapping theory was the discovery of place cells (O'Keefe and Dostrovsky, 1971). The most salient event controlling the firing rate of these cells in a freely moving animal is the location of the animal within a particular environment (O'Keefe and Conway, 1978; O'Keefe and Dostrovsky, 1971). According to the cognitive mapping 
theory, learning and memory are supported by two systems - the taxon system and the locale system. The taxon system acquires information independently of the hippocampus and is involved in habit learning or stimulus-response learning. The locale system, on the other hand, depends on the integrity of the hippocampal formation and supports cognitive mapping. The locale system, centered on the hippocampus, creates and maintains a unified map-like representation of an environment, which is essentially a representation of places, directions, and distances between them. Whenever the discrepancy between the stored map and the current perception of the environment is detected, information is updated in an all-or-none manner by exploration, in a way that is independent of goal-oriented behavior.

Also, briefly, at approximately the same time, Hirsh (1974) distinguished between two systems, one system involving performance-line storage and the second memory system involving conditional relationships between events. Only the latter he linked to the hippocampal formation. O'Keefe and Nadel (1978) along with Hirsh (1974) appear to be the first theorists to have made explicit the multiple memory system approach and applied it to explain learning and memory deficits.

Theories of associative learning, in which learning is described in terms of the associative strength among stimuli and events, as well as the error-correcting rules by which modifications of associative strengths occur (Mackintosh, 1975; Pierce and Hall, 1980; Rescorla and Wagner, 1972), provide a principled account 
of how learning, including learning about places, occurs. Another psychological theory of hippocampal function, based on associative learning principles, is Sutherland \& Rudy's (1989) configural association theory. Configural association theory (see also Rudy and Sutherland, 1985) proposes that the hippocampal formation contributes to learning and memory by storing configural representations and enhancing the activation or salience of a more limited set of configural or conjunctive representations in the cortex.

According to this theory, learning is also dependent on multiple systems. One system depends upon the hippocampus and supports the creation of complex representations. It does so by binding two or more features or cues together, which are subsequently used to solve many spatial and non-spatial memory problems. In principle, such a system could make a contribution to resolving any discrimination problem that contains a relationship between two or more cues or events. Conjoining two or more cues, which can be spatial or non-spatial in nature, forms the configural representation.

The configural association theory predicts that animals with hippocampal damage are especially susceptible to deficits in discrimination problems that require configural (non-linear) solutions (l.e. cannot be solved by the combination of the individual associative strengths of the individual stimuli comprising the compound). An example of a task that requires a configural solution is the transverse patterning task $(A+B-; B+C-; C+A-;$ which underlies the problem of "Rock-Paper-Scissors" on a more familiar (evel), a task that will 
be discussed in more detail later. Some other tasks that engage a configural solution include negative patterning ( $A+; B+; A B-)$, certain conditional discriminations ( $\mathrm{AC}+; \mathrm{B}+; \mathrm{AB}-; \mathrm{C}-)$, and bi-conditional discriminations ( $\mathrm{AC}+$; BD+; AD-; BC-; see Rudy and Sutherland, 1985 for a review). Even when problems can be solved using the summation of the associative strengths of cue representations in nonhippocampal networks, it should be clear that in the intact animal the hippocampus will construct a configural (conjunctive) representation from the same cues. Depending upon the relative rate of associative strength acquisition and the number of learning episodes, it is possible that the hippocampus could dominate performance even in problems with linear solutions.

In this regard, O'Reilly \& Rudy (2001) proposed a theory that is akin to configural theory, but with additional assumptions about the different properties of learning in hippocampal and nonhippocampal systems. The emergence of this theory was in part motivated by reports showing that animals with hippocampal damage are able to learn to solve at least some configural problems. O'Reilly \& Rudy (2001) suggest that two separate systems contribute to the formation of configural or conjunctive representations and they provide a principled basis for deciding when memory tasks would favor one system over another. One system is hippocampus-dependent. It rapidly and automatically forms and stores conjunctive (configural) representations during incidental learning. The second system is neocortex-dependent (and not hippocampus dependent), and it supports learning of simple discriminations, and can form configural 
representations. This system also supports stimulus conjunctions (configurations), but it does so gradually only if driven by task demands.

In a similar vein, Eichenbaum $(1992,2001)$ proposed a relational theory of hippocampal function. In their view, the hippocampus supports relational representations involving multiple events and plays a central role in declarative memory. Declarative memories involve conscious recollections of events and may be called upon in the future to solve novel problems. The relational theory proposes that the hippocampal relational network allows for logical inferences to be made about events or items that may have never been experienced together in the past. This representational flexibility, they claim, allows for previously learned information to be applied in novel situations involving different actions.

Evidence bearing on central predictions of this theory is not unequivocal. In particular, the sort of transitive inference process claimed to be dependent on hippocampal circuitry has been shown computationally not to require relational processing (Frank et al., 2003). Furthermore, transitive inference has been shown to be unaffected by large hippocampal lesions (Van Elzakker et al., 2003). Similarly problematic is the finding that rats with large hippocampal lesions show transfer of perfect discrimination performance to novel combinations of previously trained reinforced and non-reinforced visual cues (Driscoll et al., 2004b) in the Visual water task (Prusky et al, 2000a). 
As a final contemporary theory discussed here, Squire (1992) has presented a position on hippocampal function that has been very influential over the years. This theory proposes that there are multiple memory systems with distinct anatomical organizations and distinct functions. The hippocampus, along with anatomically related structures, is fundamental for declarative memory, that is, consciously recalled, reportable memories for events or facts. The hippocampus is involved in storing a representation that is involved in binding distributed sites in the neocortex in order to form complete, accessible memories. Squire (1992) proposes that there is a gradual process of reorganization that permits memories to be retrieved via strengthened cortical connections independently of the hippocampus. Declarative memory is contrasted with implicit (procedural) memory, which does not dependent on the hippocampus.

Taken together, these theories of hippocampal function agree that the hippocampus contributes to the binding of memories of the components of events, including the spatio-temporal contexts within which they occur. The precise processes taking place in the hippocampus remain, however, a matter of ongoing debates and the above discussion of theories of hippocampal function is by no means exhaustive. The fact that the hippocampus is essential to spatial navigation, however, is largely agreed upon.

The position that is favored here is that the hippocampal formation is essential for initial encoding, and for at least initial storage and retrieval of information. Furthermore, the loss in processing ability afforded by this system produces 
deficits in learning and memory that accompany the aging of the hippocampal formation. Thus, we are in agreement with many theories of the hippocampal function (Hirsch, 1974; Mishkin et al., 1984; O'Keefe and Nadel, 1978; O'Reilly and Rudy, 2001; Rudy and Sutherland, 1995; Sutherland and Rudy, 1989), which posit that people or animals with damage to the hippocampus solve simple discrimination problems involving unambiguous single cues by gradually incrementing and decrementing the associative value of the elemental cues, but have difficulty solving problems that require configural/conjunction solutions.

\subsection{Hippocampus-dependent Learning and Memory in Rodents}

As already mentioned, many memory tasks are sensitive to hippocampal damage. Non-human animal research provides us with a wealth of knowledge about tasks that are dependent on hippocampal integrity, permitting further exploration of structural, functional, and molecular substrates. Two tasks that have been initially employed in testing non-human animals, and subsequently applied to humans, are of particular interest to us: the transverse patterning discrimination task (TPDT) and the Morris water task (MWT); non-spatial and spatial hippocampus-dependent tasks respectively. 


\subsubsection{Spatial Navigation}

A disruption in spatial learning and memory has been repeatedly observed in animals with hippocampal damage (Morris et al., 1982; Olton and Samuelson, 1976; Sutherland et al., 1982a). The Morris water task (1981) has become the gold standard for measuring spatial learning and memory in rodents. In its most commonly used version, the Morris water task involves animals navigating to a platform hidden in a fixed location in a pool of opaque water based on learning a configuration of room cues outside the pool. Learning is assessed by measuring approach latencies or path length traveled from the release location to the platform. Both measures should improve across days and trials in a normal rat. Memory retention is often assessed during a fixed duration probe trial with the hidden platform removed from the pool. Intact animals swim over the location where the platform had been during training several times during the probe trial and have a selective, biased search pattern that reflects a preference for this region of the pool. For a rat to display a selective search bias, the rat should spend more time in the quadrant where the platform was located during training compared to any other quadrant (the same measures of performance are adopted in assessing success in human performance on virtual navigation tasks, which will be discussed below). Several types of hippocampal damage, including lesions (Morris et al, 1982; Sutherland et al, 1982a), pharmacological manipulations (Packard and White, 1991; Sutherland et al., 1982b), genetic and environmental factors (Gianoulakis, 1990; Zaharia et al., 1996), and aging (Barnes et al., 1997) are known to disrupt normal place learning and abolish 
memory for previously acquired information. Specific to the Morris water task performance, rats with hippocampal damage do not selectively search the pool during the probe trial, nor do they directly approach the platform location from the initial starting point. It is important to note that the loss of spatial navigation is independent of the disruption in motor, perceptual, or motivational components of the task (Morris et al., 1982; Morris et al., 1986).

Similarly, there are numerous reports of age-related deficits in rats on the Morris water task. Age-related deficits in performance are well documented in the standard fixed location, hidden platform version (Gage et al, 1984,1988; Gallagher and Pelleymounter, 1988; Gallagher et al., 1993; Hebda-Bauer et al., 1999; Lindner and Gribkoff, 1991; Menaey et al., 1991; Pelleymounter et al., 1987; Rapp et al., 1987), and in the working memory version (Markowska, 1999; Van der Staay and de Jonge, 1993; Wyss et al., 2000). Spatial working memory in the rat can be assessed, for example, by testing the acquisition of the hidden platform location from trial to trial so that the location of the platform is varied on each subsequent trial in the session (Frick et al., 1995), or by allowing two 1-minute training sessions and then testing 30 seconds after the second training session on a no-platform probe trial (Lee et al., 2000). Overall, aged rats are less efficient at acquiring the task compared to younger adult rats, and spatial learning and memory deficits are reported in the absence of significant sensory, motor, or motivational problems (Gallagher and Pelleymounter, 1988; Rapp et al., 1987). 
This age-related spatial deficit in the Morris water task is not unique, given that aged rats and rats with hippocampal damage also exhibit deficits in performance on other spatial tasks, such as the radial arm maze (Barnes, 1979; Barnes et al., 1989; Gallagher et al., 1985; Jarrard, 1983; Olton and Samuleson, 1976), the T-maze (Bannerman et al., 2001; Rawlins and Olton, 1982), and the Barnes circular maze (Barnes, 1979; Barnes, 1988; Barnes and McNaughton, 1985; Barnes et al., 1989; Barnes et al., 1990). In addition, it has been demonstrated that aged animals exhibiting behavioral hippocampus-dependent deficits also exhibit different hippocampal physiological and morphological profiles compared to both young and old unimpaired animals (for a review see Geinisman et al., 1995), certain aspects of which will also be considered shortly.

\subsubsection{Transverse Patterning Discriminations}

In addition to deficits in spatial tasks, rats with hippocampal damage have deficits in certain forms of non-spatial learning. In particular, they have difficulty solving configural/conjunctive discriminations, such as the transverse patterning problem, but no trouble solving similar elemental (simple) discriminations (Alvarado and Rudy, 1995a; Dusek and Eichenbaum, 1998). Elemental problems involve associating a discrete cue (e.g., A) with a response ( $X$; e.g., $A-X)$. Unlike elemental discriminations, simply responding to or avoiding a single cue cannot solve the transverse patterning problem, because each choice stimulus is ambiguous in predicting a correct response. Successful 
performance on the transverse patterning problem involves associating a compound cue with a correct response (e.g., AB-X). A compound cue is formed when multiple stimuli (e.g., A and B) are combined to form a single representation (e.g., $A B$ ), The only way to successfully solve this problem is to utilize a configural solution, which in turn requires one to respond on the basis of the relationship between the cues.

It has been demonstrated that rats with hippocampal damage are unable to learn transverse patterning discriminations, while elemental discrimination learning is spared (Alvarado and Rudy, 1995a). Also, Rondi-Reig et al. (2001) tested mice lacking CA1 NMDA receptors on the transverse patterning problem that required simultaneous acquisition of three odor discriminations comprising the transverse patterning problem contrasted with concurrent learning of three simple odor discriminations. They found that CA1 NMDA receptor knock-out mice were impaired in solving the transverse patterning odor discrimination compared with normal performance on the non-overlapping (elemental) concurrent odor discriminations (but see Bussey et al., 1998). To the best of our knowledge, prior to the present work, the transverse patterning task has not been previously employed in the studies of aged animals. 


\subsection{Hippocampus-dependent Learning and Memory in Humans}

The important role that has emerged for the hippocampus in spatial learning and memory in non-human animals has prompted examination of the human hippocampus involving similar tasks. Thus far, the evidence supports the notion that the hippocampus plays an important role in spatial learning and memory across many species. For example, damage to the hippocampal formation in species as widely divergent as pigeons and humans results in disrupted spatial learning. Also, recent neuroimaging investigations have provided evidence showing that the human hippocampus becomes activated during spatial navigation. Such studies will be discussed in detail below.

\subsubsection{Virtual Navigation}

A variety of tasks have been used to assess human spatial cognition, some likely engaging similar processes as in non-human animal experiments and others engaging very different processes. For example, mental rotation, spatial orientation, route learning, map learning, pointing to places, throwing to targets, intercepting projectiles, and measuring water levels have all been employed to measure human spatial ability. A key point in the human studies of spatial ability is that they often reveal a superior performance by males. For many, the mental rotation task originally described by Metzler \& Shepard (1974) is the gold standard for measuring spatial cognition in humans. In important 
ways, data from studies that have employed mental rotations do not lend themselves to a direct comparison with data on performance of a rat in the Morris water task. Even though both tasks are thought to be spatial in nature, evidence suggests that they involve a distinctly different circuitry (posterior parietal vs. hippocampal) and to date mental rotation performance has not been tested in the rat. A human analog of the Morris water task for measuring hippocampal processes has been developed, however, using virtual navigation through computer-generated environments. Many virtual navigation tasks, such as a virtual arena (Jacobs et al., 1998), a virtual reality town (Maguire et al., 1998), a virtual maze (Moffat et al., 1998), a virtual Morris water task (Astur et al., 1998a; Astur et al., 2002; Driscoll et al., 2003a, 2005; Hamilton and Sutherland, 1999; Hamilton et al., 2002; Hamilton et al., 2003; Moffat and Resnick, 2002), and a Hebb-Williams maze (Shore et al., 2001) have been developed to facilitate the testing of human navigational processes in a laboratory setting.

Hippocampal activation has been observed using PET in participants learning to navigate in virtual environments (Maguire et al., 1996, 1997, 1998). For example, Maguire et al. (1998) designed a clever experiment in which London taxi drivers were examined on their knowledge of complex routes versus landmarks around London. Differences in activation were seen in the parietal lobe, the cingulate cortex, the parahippocampal gyrus, and the right hippocampal formation. Further, functional neuroimaging of human brain activity during navigation in a virtual town revealed that navigational accuracy varied 
directly with the degree of activation of the right hippocampus, while the left hippocampal activation was related to non-spatial aspects of navigation (Maguire et al., 1998). Damage to the right temporal lobe (including the hippocampus) due to thermo-coagulation along the amygdalo-hyppocampal axis in an attempt to alleviate epilepsy has also been shown to affect performance on procedures that emulate rodent spatial learning (Bohbot et al., 1998). The above-mentioned experiments comprise a growing body of evidence suggesting that the human hippocampus is involved in spatial information processing. Taken together, human brain lesion and neuroimaging studies correspond well with rat lesion studies in demonstrating that hippocampal damage compromises spatial navigation in significant ways. Other involved regions (such as right caudate nucleus, right inferior and bilateral medial parietal regions, and left frontal lobe) have been implicated, outlining a distributed network supporting human navigation, and linking the functions of the network regions to physiological observations already reported in other mammals.

The computerized version of the Morris water task developed in our laboratory (Astur et al., 1998a; Hamilton and Sutherland, 1999) is part of an effort to translate non-human work into a virtual domain, in order to develop human models of rat memory processes. We have evaluated whether many psychological functions engaged by this task in rats are similarly engaged in humans (Hamilton et al., 2002). For example, we investigated human place learning in the virtual Morris water task under comparable conditions to those employed by Sutherland et al. (1987) with rats. More specifically, Sutherland et 
al. (1987) tested seven different groups of rats in the Morris water task by systematically varying the opportunity to view and/or navigate through the environment. All groups in the Sutherland et al. (1987) experiment were released in one half of the pool, while physical and/or visual access to the other half of the pool where the hidden platform was located were restricted. When subsequently tested without restrictions, only rats that were able to view and navigate through the entire environment without restrictions performed well.

Years later, the same conditions were imposed in a virtual environment on seven different groups of undergraduate students (Hamilton et al., 2002). Like the rats in the Sutherland et al. (1987) experiment, the human participants were trained to navigate to a hidden platform under the same seven conditions, but in the computerized task. Our results show that human performance in the virtual Morris water task closely matches the data obtained by Sutherland et al. (1987) with rats in all of the seven different conditions that were imposed on the subjects. Furthermore, our data indicate that there is some generality in the principles involved in place learning and provide a reasonable basis for crossspecies comparisons (Hamilton et al., 2002).

In addition, we propose that the virtual Morris water task not only allows greater control over space than would be possible in a real-world navigation task, but that it, to an extent, taps similar processes to those engaged by the task in the rat. Thus, it enables one to draw direct connections to an existing body of animal research in this area, a literature that includes levels of description of 
spatial memory processes involving specific networks, single neuron physiology, and molecular signaling. One may imagine making intimate contact between human cognitive research and the vast amount of animal literature on hippocampal function that is usually overlooked when designing human studies.

Even though some might argue that a meaningful comparison would be inappropriate due to the inherent limitations of virtual environments, such as the lack of vestibular and proprioceptive cues, the work conducted in our (Hamilton et al., 2002) and other laboratories (Shore et al., 2001) provides a reasonable basis for believing that valid cross-species comparisons can be made using such tasks. So far, the virtual Morris water task has proven useful in studying sex differences in place learning and memory (Astur et al., 1998a, 2004; Driscoll et al., 2005), rules governing place learning (Hamilton and Sutherland 1999; Hamilton et al., 2002), deficits after hippocampal damage (Astur et al., 2002), aging (Driscoll et al., 2003a, 2005; Laurance et al., 2002; Moffat and Resnick, 2002), and fetal alcohol syndrome (FAS; Hamilton et al., 2003). Together, these studies demonstrate that successful spatial navigation in humans, as assessed by virtual navigation, is also highly dependent on the integrity of the hippocampal formation.

In a relatively direct test of this idea, Astur et al. (2002) tested ten patients with unilateral resection of the hippocampus on a virtual version of the Morris water task. Five of the patients had the left and five had the right hippocampus removed. They found that patients with hippocampal resections display severe 
deficits in spatial navigation compared to the age-matched controls and agematched patients with extra-hippocampal resections, suggesting that the hippocampus is critical for spatial learning and memory regardless of which hemisphere was damaged. In addition, a preliminary evaluation of performance in a VMWT with functional magnetic resonance imaging (FMRI) by Astur et al. (2002) revealed a bilateral activation centered in the hippocampus, consistent with other functional imaging reports during virtual navigation (Aguirre et al., 1996; Maguire et al., 1998).

Similarly to aged rats, aged humans are thought to have spatial information processing deficits (Kirasic and Allen, 1985; Perlmutter et al., 1981). Recent evidence indicates that elderly people are impaired in spatial navigation in virtual environments (Driscoll et al., 2003a, 2003b, 2005; Laurance et al., 2002; Moffat and Resnick, 2002; Moffat et al., 2001). Taken together, the human imaging studies are consistent with the idea that the hippocampal formation contributes to the encoding and retrieval of topographical information in humans. Furthermore, human imaging and lesion studies compare well with non-human animal studies that show robust and persisting deficits in spatial ability following hippocampal damage. 


\subsubsection{Transverse Patterning Discriminations}

The transverse patterning discrimination problem is an important foundation for evidence supporting the configural/conjunctive association view of hippocampal function. Although there are reports that adult humans are unable to solve the transverse patterning (Berch and Israel, 1971), contrasting evidence offered by Astur and Sutherland (1998b) indicates that college-aged individuals have little difficulty solving this problem, especially if a stepwise approach is used as previously described by Alvarado and Rudy (1995a) with rats. Further, Astur and Sutherland (1998b) suggest that participants seem to adopt a configural strategy when presented with ambiguous stimulus pairs, even though an elemental strategy would suffice. Also, Rudy et al. (1993) reported that children at least 4.5 years old were able to solve transverse patterning and conditional discriminations, two problems that require configural association solutions (i.e., are hippocampus-dependent). In addition, they reported that younger children did not solve these problems, but were able to solve problems that permitted an elemental solution even though the problems were constructed from the same stimulus materials. They suggest that children may not gain access to configural solutions until they are about 4.5 years old and that the configural association system depends on different, later developing neural structures than does the elemental association system.

A more recent study (Astur and Constable, 2004) attempted to assess the role of the hippocampal formation on transverse patterning discriminations using 
functional magnetic resonance imaging. Astur and Constable (2004) report that activation maps and time course data both suggest that the hippocampus is involved in this task, but interestingly, the hippocampus was less active during the hippocampus-dependent period of the testing phase compared to both the hippocampus-independent test phase and the fixation control phase. This should alert us that the traditional assumptions advocating that the increase in bloodoxygenation level dependent response is necessary in order for a brain region to be considered involved in a task might be inaccurate and that alternative explanations, such as the hippocampal 'dampening' in this case, should be considered. Impairment in solving the transverse-patterning problem has also been reported in amnesic patients with medial temporal lobe damage (Reed and Squire, 1999; Rickard and Graffman, 1998). 


\section{The Aging Hippocampus}

The hippocampal formation is often implicated in the age-related cognitive decline. There is agreement that many behavioral deficits exhibited during normal aging resemble the ones seen following bilateral disruption of the hippocampus (Geinisman et al., 1995). Important features of the age-related cognitive impairment include that, once acquired, the information is lost rapidly, and also that newly learned information is much more vulnerable compared to the information learned in the remote past. While qualitatively similar, the agerelated deficits are, however, believed to be quantitatively less severe.

An advantage of the aging rodent studies is in the ability to separate and strictly control the contribution of genetic and environmental factors. As one may imagine, this is much harder to do with human participants. Nonetheless, many factors have been proposed as a neural substrate for age-related cognitive decline. Here we consider only a subset of them, namely physiological, structural, and biochemical changes.

\subsection{Physiological Changes}

One possible physiological source of age-related impairments in hippocampusdependent learning and memory that has been extensively investigated is the mechanism of long-term potentiation (LTP), also sometimes referred to as long 
term enhancement (LTE). LTP is a relatively enduring form of synaptic plasticity that is well represented in the hippocampus. It is presently the best candidate for the neural mechanism that underlies information storage in the mammalian brain. It is presumed that the "activity-dependent synaptic plasticity is induced at appropriate synapses during memory formation and is both necessary and sufficient for the information storage underlying the type of memory mediated by the brain area in which the plasticity is observed" (Martin et al., 2000). Therefore, the change in the strength of connections between the neurons is assumed to be the mechanism by which memory traces are encoded and stored in the brain. So far, there is evidence that older rats show impairments in LTP induction in the Schaffer-collateral-CA1 synapse when sub-optimal pulse parameters are used. It has also been demonstrated that a larger amplitude current is necessary for LTP to be induced at the perforant path granule cell synapses (Barnes et al., 2000). Together, these observations point to a change in the induction threshold at the synapse, and that LTP should be harder to induce in older rats. Age-related changes in LTP are often attributed to the reduction in the number of perforant path synaptic contacts on granule cells. A number of factors have been suggested to account for differences in synaptic plasticity in aged rats, such as possible age-related differences in protein synthesis, axonal transport, calcium regulation, and hippocampal synaptic interconnectivity. 
It is also possible that different regions within the hippocampus differentially undergo physiologically detectable changes with aging (Rosenzweig and Barnes, 2003). Overall, impairments indicative of alterations in neural plasticity are observed in a number of aged animals, specifically those that exhibit poor memory function. It is easy to see how such changes in network connectivity could be responsible for changes in plasticity with advanced age. Many reports by Barnes and colleagues (Barnes, 2003; Rosenzweig and Barnes, 2003) suggest, however, that old rats can display intact LTP induction but that the enhancement decays significantly faster. Disparate results like these in the aging and LTP literature are often attributed to critical methodological differences, such as the intensity of stimulation or whether induction or decay is measured. Sometimes even the strain of rat has been considered as a possible source of differences.

The present overview merely scratches the surface when it comes to the topic of hippocampal synaptic plasticity and aging, in part because this has been the subject of excellent recent reviews (for example see Barnes, 2003; Geinisman et al., 1995; Rosenzweig and Barnes, 2003), Rosenzweig and Barnes (2003) not only review the work on synaptic plasticity and network dynamics in relation to aging but also the available evidence for the role of LTP in learning and memory. 


\subsection{Structural Changes}

Age related structural changes that are likely to be relevant to hippocampusdependent cognition have been identified. Regional differences in the aging brain are thought to be rather subtle in comparison to age-related global brain deterioration. Some evidence suggests that the hippocampal formation may be particularly vulnerable to the effects of aging, especially age-related pathology. This evidence includes reports of decrease in neuronal count, intracellular pathology, affinity of neurofibrillary tangles for this region ( $\operatorname{Raz}, 2000$ ), and a decrease in volume (Golomb et al., 1993; Jernigan et al., 1991). Age-related reductions in hippocampal volume have been observed repeatedly (Golomb et al., 1993; Jernigan et al., 1991), but there are also important exceptions that should be noted (Raz, 2000; Sullivan et al., 1995). Despite the equivocal findings at times, it is our position that it is doubtful that the hippocampal formation would completely escape the deleterious effects of old age, especially when one considers its involvement in age-related pathology.

In vivo neuroimaging reveals only mild age-related shrinkage of the broadly defined hippocampal regions. Traditionally, volume loss was considered to be a direct result of neuronal death. Evidence indicates that observed changes might be due to the shrinking of neurons rather than their death (Esiri, 1994). Cytoarchitectonically, the hippocampus is a complex structure and one might expect that different subdivisions of the formation may be differentially affected 
by age. Evidence from some in vivo studies of the hippocampus suggests a possible "anterio-posterior gradient of age-related vulnerability" (Raz, 2000). Petersen et al. (1998) demonstrated that the anterior hippocampus, unlike the body and the posterior part, is less likely to atrophy in early $A D$ patients and non-AD controls with age-related memory problems. Further, hippocampal volumetric studies in which anterior hippocampus was not measured were more likely to show an age-related volume decline than the studies that included less vulnerable anterior slices (Raz, 2000). In turn, the functional distinction along the anterio-posterior axis of the hippocampus is of particular interest.

In comparing highly specialized spatial navigation in London taxi drivers with that of control subjects, Maguire et al. (2000) found a positive correlation in the volume of posterior hippocampus and negative correlation in the volume of anterior hippocampus with the amount of time spent as a taxi drive. The same associations were found with their performance on a virtual navigation task. The results are in agreement with the idea that the posterior hippocampus is responsible for processing related to efficient usage or storage of spatial information. Similarly, there is some evidence that the posterior hippocampus in monkeys (Colombo et al., 1998) and the dorsal hippocampus in rodents (homologous with the posterior hippocampus in monkeys and humans) (Moser and Moser, 1998; Moser et al., 1993) are more important than the anterior (or ventral) regions for encoding spatial information. 
MRI-volumetric measurements are being used clinically with increased frequency. It has been reported that the hippocampal volume accurately reflects the structural-functional relationships between memory deficits and hippocampal damage from normal aging to dementia (Petersen et al., 2000). The appeal of this technique is that, clinically, a relevant brain structure can be measured in vivo, and that this information may aid in diagnosis of pathology early on.

Structural MR imaging is seldom performed on non-human animals. In nonhuman animals, morphological analyses are customarily performed on postmortem tissue. Changes in the number of hippocampal neurons and synapses have been extensively investigated over the years in hope of finding structural substrates of age-related memory decline. Many, but not all, have reported a loss in the hippocampus in the number of neurons and/or synapses that is associated with old age (for a review see Gallagher and Rapp, 1997). Additionally, this structural loss has been correlated with the severity of memory impairments in the same animals. Few of such studies have, however, employed unbiased counting techniques. When such techniques were employed, agerelated alterations in neuron and synapse number were only convincingly demonstrated in humans, and not in the non-human animals with age-related memory deficits.

We propose that MRI provides a valuable technique in bridging the gap between humans and non-human animals in that structural integrity can be measured in non-human animals in vivo employing the same techniques that are used in 
human research. In addition, the non-human MR results can be easily complemented with cellular and molecular post-mortem measurements of the same tissue. Even though structural research on non-human animals has been extensive, the progress in technique development makes this research area a fertile ground for further exploration.

\subsection{Biochemical Changes}

The precise nature of the affected processes that lead to the age-related decline in memory is still uncertain, even though definitive age-related physiological and anatomical changes have been reported. A complimentary technique to MRI is magnetic resonance spectroscopy (MRS). MRS uses tools similar to the more familiar magnetic resonance imaging (MRI) to measure brain function in vivo. In the past ten years MRS has carved out a niche in the evaluation of metabolic processes in the living organism. MRS allows the measurement of the concentrations and synthesis rates of individual chemical compounds within a precisely defined brain region. Most common measurements are based upon the IH nucleus, and most often studied measurable metabolites include $\mathrm{N}$ acetylaspartate (NAA), creatine (Cre), choline (Cho), $\gamma$-amino butyric acid (GABA), glucose, glutamine, glutamate, and lactate. These metabolites play critical roles in neuroenergetics, neurotransmission, and neuromodulation (Nicholls, 1989; Sokoloff, 1999). Often metabolic alterations can be detected before the more obvious structural alterations become apparent, both in 
response to treatment and as a part of natural progression of the disease. By employing MRS, it is possible to measure levels of different brain metabolites in vivo in a particular region of interest. The application of proton magnetic resonance spectroscopy $\left({ }^{1} \mathrm{H}\right.$-MRS) to the investigation of the brain most often involves measuring $\mathrm{N}$-acetylaspartate (NAA), choline (Cho), and creatine (Cre) containing compounds.

A decrease in NAA concentrations was classically believed to reflect atrophy, neuronal loss, and/or decreased neuronal density. Recent studies, however, suggest that lower levels of NAA/Cho do not seem to be necessarily related to neuronal loss, but may also be associated with metabolic dysfunction (Seilhean et al., 1993). For example, this appears to be the case in dementia complex in HIV patients with documented neuronal injury (Lipton, 1992). Consequently, it has been suggested that decrease in NAA values may also reflect the magnitude of neuronal injury and metabolic dysfunction. Decrease in NAA in the hippocampus has been reported in several conditions, including PTSD (Schuff et al., 1997a), epilepsy (Ende et al., 1997), Alzheimer's disease (Schuff et al., 1997b) and normal aging (Driscoll et al., 2003a, 2005; Schuff et al., 1999).

NAA is a putative neuronal marker since it is almost exclusively found in neurons (Moffet et al., 1991) and is virtually undetectable in other cell types (Urenjak et al., 1993). Nonetheless, the role of NAA in neuronal function and hence the exact significance of MRS measurements of NAA are still uncertain despite the fact that NAA is one of the most abundant amino acids in the brain. NAA has 
been suggested to be a source of acetyl groups for lipid synthesis, a regulator of protein synthesis, a storage form of acetyl-CoA or aspartate, a breakdown product of $\mathrm{N}$-acetyl-aspartyl-glutamate, or an osmolite (Birken and Olendorf, 1989). Animal models of chronic neuronal injury provide reliable correlations between NAA levels and in vitro measures of neuronal survival, tempting one to equate NAA with neuronal density or to label it as neuronal marker. On the other hand, there are reports of reversible NAA deficiency due to either spontaneous or treatment-related recovery, suggesting that the initial drop could not be due to the loss of axons or neurons.

Reversible decrease in NAA has been reported in various conditions involving brain injury (Davie et al., 1994; De Stefano et al., 1995; Hugg et al., 1996; Pavlakis et al., 1998), making the role of NAA as a specific marker uncertain. Also, Demougeot et al. (2001) reported that cellular dysfunction can cause greater reductions in NAA levels than the neuronal loss itself. Furthermore, their findings suggest that dysfunction in mitochondrial energy metabolism may be sufficient for a severe drop in NAA. Such observations suggest NAA to also be a marker of cellular dysfunction in certain cases. Moreover, elevated NAA levels are found in Canavan's disease, and clearly do not reflect an increase in neuronal density here, but rather the deficiency in an enzyme (aspartoacylase) responsible for NAA degradation. Taken together, the data indicate that NAA levels probably underestimate neuronal density. Clearly, NAA quantification does not provide a faithful estimate of neuronal density and should not replace histological examinations. NAA remains useful in assessing early cellular 
dysfunction before histological lesions appear. Still, NAA does not appear to be a sensitive marker of neuronal density and other complementary assessments should be used before coming to such conclusions, and reversible cellular dysfunction must be considered as a plausible explanation.

Choline (Cho) resonance originates from glycerophosphocholine, phosphocholine, and choline itself, which are all integral components of membrane phospholipids. Increased Cho levels are taken as a sign of membrane breakdown, inflammation or demyelination as seen in stroke (Olson et al., 1992) and multiple sclerosis (Davie et al., 1994). Creatine (Cre), whose resonance represents the sum of creatine and phosphocreatine, provides a measure of cellular energy currency. Choline and creatine are found in all brain cells, making the changes somewhat difficult to interpret and understand.

Many MRS studies in humans report general age-related alterations of the aforementioned metabolites (Chang et al., 1996; Fukuzako et al., 1997), whereas others are more specific to hippocampal metabolite changes associated with volume loss (Schuff et al., 1999). Studies in different patient cohorts (Brooks et al., 1997, 1999; Jung et al., 1998) and normal populations (Jung et al., 1999) using quantitative spectroscopy and an extensive neurocognitive battery reveal a significant relationship between neurochemical markers of brain integrity and brain function. For example, NAA was related to the overall performance on neuropsychological battery of tests, especially the timed ones, in normal young adults (Jung et al., 1999) and in patients with systemic lupus 
erythematosus (Brooks et al., 1999). Despite the limitations, not just in the method itself but in the interpretation of results, we believe ${ }^{1} \mathrm{H}$ MRS/MRSI to be a useful tool in assessing hippocampal neuronal integrity with aging. Furthermore, proton MR spectroscopy may provide a clue in determining a relationship between age-associated brain atrophy and brain function.

An increasing number of techniques are being developed to non-invasively analyze structural, physiological, and chemical changes of the brain in vivo. MRS has a unique value in the realm of different imaging techniques - it provides information on a quantitative spectrum of multiple compounds simultaneously in the region of interest. The application of MRS in investigations of various disease progressions is increasing, and so is the knowledge on the unique biochemical profiles of different neurodegenerative disorders.

Overall, non-invasive MRS measurements of NAA appear to be one of the best surrogate markers currently available for neuronal integrity. A deeper understanding of NAA biochemistry should help elucidate its function in normal and pathological states. Until then, ${ }^{1} H$ MRS spectrum of NAA and other metabolites have proven at times to be effective indicators of neuronal/axonal viability and are finding an increasing application in research and in clinical neurology, 


\subsection{Neurogenesis}

In discussing the hippocampus we find it difficult to ignore the topic of adult neurogenesis. We will, however, only scratch the surface on this topic here. Adult neurogenesis seems to be restricted to two regions in the brain, one of which is the dentate gyrus of the hippocampus. Demonstration of neurogenesis in adult brain represents a major advance in our understanding of the cellular mechanisms underlying neuronal circuit plasticity and complex behavior.

The traditional view of the mammalian brain organization is that the brain becomes structurally stable shortly after birth, and no new neurons are added throughout adulthood. More recently this view has been supplemented by an appreciation of the importance of regressive events in sculpting mature circuitry postnatally, including neuron and synapse loss. Versions of this view have for centuries guided research on neurodegenerative diseases, brain injury, and recovery of function. A challenge has arisen in the last decade with the development of new methods that yielded evidence of adult neurogenesis (Cameron and McKay, 2001; Kuhn et al., 1996). The studies corroborated the early reports (Altman and Das, 1965; Kaplan and Hinds, 1977), which were largely ignored and had little impact on the field. There are at least two brain regions in which neurogenesis in adult animals has been demonstrated unequivocally: a forebrain subventricular zone (SVZ) and the subgranular zone (SGZ) in the dentate gyrus of the hippocampus. 
Currently, there is agreement that granule cells in the SGZ continue to proliferate, migrate, and fully differentiate throughout adulthood in the hippocampus of every species investigated so far, including birds (Barnea and Nottebohm, 1994), mice (Kempermann et al., 1997), rats (Bayer, 1982; Cameron et al., 1993, Derrick et al., 2000), tree shrews (Gould et al., 1997,), monkeys (Gould et al., 1998, 1999b; Kornack and Rakic, 1999), and humans (Eriksson et al., 1998). Although the exact function of adult neurogenesis is unknown, several studies suggest that it is likely to play a role in the normal information processing in the circuits to which neurons are added and as such would play an important part in hippocampal plasticity. Some have also suggested that newly generated neurons in the dentate gyrus may play a role in certain aspects of learning and memory.

The inability to generate replacement neurons has long been considered to be an important cause of neurological disease, age-related symptoms, and behavioral impairments. This raises the possibility that naturally occurring neurogenesis may be useful for slowing the progression of functional loss in the aging brain. The production of new neurons is only one of several steps, however, believed to be necessary to restore damaged circuits. More importantly, once born and differentiated, these neurons need to survive and become a functionally integrated part of their neuronal environment. Studies of normal animals have identified several factors that can affect the production and survival of new neurons in the adult brain. 
Regulation of the rate of neurogenesis is controlled by a variety of behaviorally derived factors and endogenous regulators. It has been suggested that growth factors through their ability to promote neurogenesis may have a role in the treatment of age-related neurodegenerative disorders by stimulating proliferation of neuronal precursors in the adult brain. Although growth factors are numerous, whether and how the aged brain remains responsive to these factors is not well known. For example, basic fibroblast growth factor (bFGF or FGF-2) is one of the primary mitogens used for neural stem cell propagation in vitro and is hypothesized to be important in controlling in vivo cell proliferation. Indeed, repeated application of FGF-2 promotes generation and maintenance of neurons from the adult human subventricular zone (Goldman, 1998). Recently it has been reported that FGF-2 delivery into the rodent brain increased proliferation of progenitor cells in the dentate gyrus and stem cells in the subventricular zone (Jin et al., 2003).

Another growth factor, erythropoietin (an inducible cytokine), appears to act directly on neural stem cells by promoting the production of neuronal progenitors at the expense of multipotent progenitors, and is found to regulate neurogenesis in vitro and in vivo (Shingo et al., 2001). In fact, erythropoietin induced a two- to three-fold increase in the rate of neuron production. It is beyond our scope to exhaustively review all of the neurotransmitters, hormones, modulators, and neurotrophins that affect adult neurogenesis. Instead, we briefly discuss below only a couple that are directly relevant to aging. 
There are also numerous reports showing that living in an enriched environment enhances the survival of newly generated cells in the hippocampus (Barnea and Nottebohm, 1994; Kempermann et al., 1997, 1998; Nilsson et al., 1999), providing a neural substrate for "use it or lose it" hypothesis. Increased rate of neurogenesis accompanied by better learning, increase in exploratory behavior, and in locomotor activity have all been reported in aged mice living in an enriched environment (Kempermann et al., 2002). Similarly, environmental enrichment enhanced the survival of newly born neurons in the adult mouse dentate gyrus (van Praag et al., 1999). Together these studies suggest that the aging hippocampus retains a potential for cellular plasticity, and that experience or activity can influence the functional status of circuitry in this region possibly via an effect on neurogenesis.

The exact function of hippocampal neurogenesis in the normal brain is still not known. Nevertheless, it has been suggested that the adult neurogenesis in the hippocampus promotes learning and memory. Correlational evidence is accumulating that supports a role for neurogenesis in compensating for dying neurons, thereby protecting the functional integrity of hippocampal circuitry (Snyder et al., 2001). Aging affects hippocampus-dependent cognitive ability, and age-related spatial memory impairments have been related to alterations in hippocampal plasticity (Barnes, 2003). These cognitive deficits can be ameliorated by the infusion of either nerve growth factor or insulin-like growth factor. Given that both of these treatments have been shown to increase 
neurogenesis in the adult dentate gyrus (Poe et al., 2001), this may be a basis for improved memory performance.

Recent data suggest that the failure in regulation of hippocampal neurogenesis might result in diminished capabilities of the hippocampus to adjust to a novel environment and to form new memories. For example, Kempermann et al. (2002) tested ten strains of recombinant mice, some known to be good learners and have high baseline levels of neurogenesis ( $\mathrm{C} 57 \mathrm{BL} / 6)$ and some which are known to be poor learners and have a low baseline levels of neurogenesis $(\mathrm{DBA} / 2)$, in order to examine whether the baseline levels of neurogenesis predict performance on the Morris water task. There was a significant correlation between the number of newly generated neurons in the dentate gyrus and the acquisition measures on the Morris water task for all the strains, suggesting specific neurogenic involvement in the function of the hippocampus - the acquisition of new information in particular. Similarly, it has been demonstrated that spatial memory performance of aged rats predicts levels of hippocampal neurogenesis (Drapeau et al., 2003).

In addition, it has been suggested that some age-related disorders, such Alzheimer's disease (Haughey et al., 2002; Jin et al., 2004; Tatebayashi et al., 2003; Wang et al., 2004), and Huntington's disease (Curtis et al., 2003; Tattersfield et al., 2004), are related to alterations in neurogenesis. Even though hippocampal neurogenesis declines with age, it remains regulated in response to external challenges. Some growth factors can be administered systemically to 
stimulate neurogenesis (Aberg et al., 2000; Wagner et al., 1999), suggesting a relatively non-invasive means for exploring this approach to treatment. This possibility that hippocampus can be stimulated to produce new cells with the ability to replace dysfunctional or lost neurons could have important therapeutic implications of great significance to the aging population. Before neurogenesis can be harnessed to ameliorate the age-related deficits, however, its role in the normal brain must be understood.

Currently there is no direct evidence suggesting that new neurons can aid in retarding the age-related functional decline, nor have there been direct prior tests. Furthermore, the enhancement of generation of new neurons has not been causally linked to recovery of lost function. For neurogenesis to be advantageous, the new neurons have to be able to not only differentiate and survive, but also to appropriately integrate into the existing neural circuitry. Once integrated, the new neurons should display functional properties similar to those of the neurons they had replaced, and should be generated in numbers that are comparable to the number of neurons that were lost.

So, are these newly generated cells functional? There is mounting evidence that suggests that the newly generated cells are indeed functional. For example, we know that adult neurogenesis produces cells that are incorporated into the correct location, express granule cell-specific antigens, have normal dendritic arbors, and extend mossy fiber type axons into CA3 field - all characteristics of mature granule cells (Kempermann et al., 2002; Stanfield and Trice, 1988). In addition, van Praag and colleagues (2002) demonstrated in hippocampal slices 
that weeks after birth new granule cells expressed the electrophysiological properties of mature granule cells. There is also a report of entorhinal cortex neurons infected with transynaptically transported pseudorabies virus that later localized to new granule cells and CA3 cells (Carlen et al., 2002), supporting functional integration into the hippocampal network. Similarly, taking advantage of the activity-dependent expression of c-fos, over $80 \%$ of new granule cells show c-fos activation by training in the Morris water task (Jessberger and Kempermann, 2003).

Collectively, there are good reasons to lead us to believe that new granule cells participate in information processing within the hippocampal network. While none of the functional properties of mature granule cells have been demonstrated in the new ones, we do have a solid foundation for pursuing functional experiments related to restoration of damaged hippocampal circuits. For a more in-depth discussion regarding hippocampal neurogenesis we refer the reader to a couple of recent reviews (Goldman, 1998; Lie et al., 2004). 


\section{Human Model of Rat Aging}

The results of a great deal of research have shown alterations in the hippocampus related to the process of aging in both humans and non-human animals. On the other hand, there are important shortcomings associated with this field of research. One such shortcoming is that the age-related alterations are often investigated in the absence of information from the participants about their level of behavioral or cognitive functioning and it is not clear how they relate to learning and memory dysfunction. Also, the tests used to assess learning and memory in humans typically are very different from those employed to test non-human animals, making it hard to generalize or make comparisons. For example, human tests are almost always verbal. Furthermore, methodologies employed between the species are often quite different and do not lend themselves to direct cross-species comparisons. For example, nonhuman animal studies are commonly performed on post mortem tissue, while studies on humans are almost always performed in vivo and often involve imaging. All in all, we are left with numerous reports on human aging, and just as many if not more reports on non-human animal aging, but despite the abundance of information there are clearly missed opportunities for the two avenues of research to inform one another.

In order to address some of these shortcomings and to further investigate agerelated changes in hippocampus-dependent cognition we set out to develop a human model of animal aging. In addition, we searched for converging evidence 
of changes that may underlie age-related deficits in hippocampus-dependent learning and memory. To that end, we conducted a study (Driscoll et al., 2003a) involving young and elderly human participants that employed the virtual Morris water task and the transverse patterning discrimination task, a spatial and nonspatial hippocampus-dependent task respectively. In addition, magnetic resonance imaging (MRI) and magnetic resonance spectroscopic imaging (MRSI) were used on the same participants to measure structural and neurochemical integrity of the hippocampus respectively.

Specifically, 16 young $(M($ age $)=26.1)$ and 16 elderly $(M($ age $)=77.6)$ volunteers participated in the experiment, 8 males and 8 females in each group. Elderly participants were recruited from the New Mexico Aging Process Study (NMAPS; Garry et al., 1982, 1992). Individuals with overt clinical conditions, such as coronary heart disease, significant peripheral vascular disease, insulindependent diabetes, hepatic disease, history of internal cancer requiring surgery, $x$-rays or chemotherapy in the past 10 years, a positive test for hepatitis, untreated hypertension, and those taking prescription medications except for thyroid and estrogen replacement, were excluded. As a part of the NMAPS, participants were also annually assessed on a battery of measurements of cognitive and physical function, health habits and attitudes, morbidity, number of falls, diet, physical activity, nutritional status, and body composition. In addition, participants were genotyped for Apoliporprotein E (APOE) $\varepsilon 4$ allele to assess genetic predisposition for developing Alzheimer's disease. Only 
participants that were not genetically predisposed to Alzheimer's disease were chosen for this study, all were APOE $\varepsilon 3$ homozygotes.

We found age-related deficits in performance of the elderly, relative to young participants on the VMWT consistent with well established reports of age-related decline in hippocampus-dependent cognition noted in numerous previous studies of aging. More specifically, elderly exhibited deficits in place learning in the virtual Morris water task as measured by latency and path length traveled to reach the hidden platform during training. Elderly participants also exhibited memory deficits compared to young participants, assessed by the time spent searching and path length traveled in the training quadrant of the pool on the no-platform probe trial of the VMWT. We found no differences in performance between young and elderly participants on conditions that were independent of the hippocampal integrity, such as the visible platform trials of the VMWT. This last finding also suggested that the elderly were able to successfully interact with the computer and navigate about the virtual environment despite their more than likely limited computer experience.

We also suggested that the observed deficit in performance on the VMWT was not solely caused by the inherent constraints of the virtual environment, such as the lack of locomotor-based proprioceptive and vestibular cues and a narrower filed of view, since all of our participants were deprived of such information. The observed deficits in spatial navigation were not sensory-motor or motivational in 
nature either given that there were no differences in the speed of swimming or in the performance on visible platform trials.

Cross-sectional experiments with just the young and old groups, however, can possibly lead to erroneous conclusions drawn about the shape of underlying aging functions. Ideally longitudinal studies would be conducted to address such questions, but longitudinal studies are often precluded by inherent constraints, such as a time commitment, high costs, and high participant attrition. Care should be taken to at least include multiple age groups in order to obtain relevant information about the shape of the aging curve for a particular measure. We conducted a purely behavioral study to assess spatial navigation across adulthood (Driscoll et al., 2005). In this study we tested young (20-39 years of age; $N=24$ ), middle-aged ( $40-59$ years of age; $N=23$ ), and elderly (60-85 years of age; $N=23$ ) adults on the VMWT. The young group performed quite well, which was expected. Surprisingly, the deficit already became apparent in the performance of the middle-aged group, and the performance of the elderly group was additionally worse. Results from our cross-sectional study suggest that, on the VMWT, performance declines linearly with age.

The same elderly participants also exhibited a deficit on the non-spatial hippocampus-dependent task, the transverse patterning discriminations, while performance on discriminations (elemental) not dependent on the hippocampus was spared (Driscoll et al., 2003a). More specifically, only $19 \%$ of elderly were able to learn the relationship among transverse patterning cues compared to 
$100 \%$ of younger adults. On the other hand, $94 \%$ of elderly and $100 \%$ of younger adults were able to successfully complete the elemental discriminations, which do not depend on hippocampal integrity. Elderly people also made significantly more errors and required significantly more trials to complete the task. Moreover, the performance on the virtual Morris water task predicted performance on the transverse patterning discriminations, suggesting that the observed behavioral age-related deficits are hippocampus-dependent rather than unique to a specific memory process (such as spatial memory per se) or related to a specific test format.

Again, in order to obtain relevant information about the shape of the aging curve for performance on the transverse patterning task, we tested young (ages 20-39; $N=24$ ), middle aged (ages $40-59 ; N=23$ ), and elderly ( $>60 ; N=23$ ) participants (Driscoll et al., 2004a). Our findings suggest that young and middleaged adults have little trouble solving the problem, while elderly participants are significantly impaired (Driscoll et al., 2004a). All three groups had no problems solving elemental (hippocampus-independent) discriminations. We find this observation especially interesting in light of our previous findings with the VMWT suggesting that performance on the spatial task seems to decline linearly with age (Driscoll et al., 2005); curiously a pattern that is different from our findings with the transverse patterning task.

In this study, we have also attempted to answer whether normal aging is associated with decrease in hippocampal volume, and if so whether there is a 
relationship between volume and hippocampus-dependent cognition (Driscoll et al., 2003a). Participants underwent MR imaging in order to obtain structural images, which were to further serve for quantitation of hippocampal volumes. Our structural findings indicate a reduction in hippocampal volume with age and are consistent with many earlier reports (for a review see Raz, 2000). In addition, the volume of the hippocampus correlated with performance on hippocampus-dependent tasks, both spatial and non-spatial. Specifically, larger hippocampi were associated with more time spent searching in the correct quadrant during the probe trial on the VMWT, and to the lower number of errors committed while learning the transverse patterning discriminations.

In assessing age-related changes in hippocampus-dependent cognition, we also wanted to examine the underlying hippocampal biochemistry and relate it to hippocampus-dependent cognitive performance in these elderly participants. We found lower NAA/Cre values in the hippocampi of our elderly participants as measured by MRSI. Cho/Cre remained stable in both the frontal white matter and the hippocampus. Thus, the results seem to be primarily driven by the decrease in NAA and not in the variation in Cho and/or Cre containing compounds. Significantly lower hippocampal, compared to frontal, NAA/Cre indicated that the observed reduction in hippocampal NAA was not a consequence of global age-related metabolic brain deterioration. Also, we found no differences in metabolic profiles between the two groups in the frontal white matter. In addition, we found that better performance on both hippocampusdependent tasks was significantly correlated with higher hippocampal NAA/Cre. 
Our combined behavioral and imaging study of a carefully selected aging sample sheds some light on hippocampal aging, as well as the neurobiological substrates of human hippocampus-dependent learning and memory. We have detected age-related variations linked specifically to the hippocampus, even though regional brain differences caused by aging are thought to be rather subtle compared to global brain deterioration.

Despite the fact that we developed a human model of animal aging using tests that allow for cross-species comparisons, and described hippocampal aging on several different levels of investigation, several questions arose. Specifically, one question that is often linked to MRI research and volumetry is related to what a decline in volume of a structure really tells us? Does it denote atrophy? Or is it simply a reduction in size? In addition, in respect to MRS, more and more the functional role of NAA in the brain is questioned? Is the view of NAA as a putative neuronal marker useful in interpreting neurospectroscopic results, or is such a view an oversimplification?

In order to address some of the questions arising from the previous studies, but also to validate and better develop our animal model of aging, and to better describe the changes that occur with age specifically related to the hippocampus we propose a multilevel analysis in the rat. 


\section{Experiment 1 - Visual Acuity}

\section{Assessment of visual acuity in the Fisher $344 \times$ Brown Norway hybrid strain of rat. Does visual acuity vary with age?}

\subsection{Background}

Animal models are helpful for gaining insight into normal aging and for determining the contribution of various factors to age differences in behavior. A specific advantage of rat models of aging is that the contribution of genetic and environmental factors can be strictly controlled and thus systematically investigated. Recently, the degeneration of the visual system with age and the effect it may have on the performance of tasks with a visual component are receiving increased attention (van der Staay, 2002). A worry is that poor performance in mnemonic or cognitive tasks often exhibited by aged rats is possibly confounded by poor vision.

Vision-mediated behavioral tasks, such as the Morris water task (Morris, 1981), the radial arm maze (Walker and Olton, 1979), and the Barnes circular maze (Barnes, 1979) are the most often used tests of spatial learning and memory in the rat. Oftentimes the results of these tests are interpreted without specific knowledge of the visual capacity of the rodent strain used. This omission is in part due to inability to test visual acuity in the rat. 
Recently, however, Prusky and colleagues (2000a, 2004) developed two tasks that allow assessment of the visual acuity in rats and mice, the grating acuity test (Prusky et al., 2000a) and the virtual optomotor system (Prusky et al., 2004).

Previously, Prusky et al. (2002) measured grating acuity of three pigmented and three albino laboratory strains, and a wild strain. The acuity of the albino strains was the lowest, around 0.5 cycles per degree (cpd). The Dark Agouti, LongEvans and wild strains clustered around $1.0 \mathrm{cpd}$. Fisher $344 \times$ Brown Norway (FBNF1) hybrid rats, however, had a significantly higher acuity threshold, around $1.5 \mathrm{cpd}$. In addition, it has been reported that rats with reduced visual acuity exhibit a significant deficit in place learning, although eventually they can reach the same latencies as the rats whose vision is not compromised (Prusky et al., 2000b), Visually impaired animals were not impaired in locating the visible platform, suggesting that the visible platform trials, which are often considered a control condition for sensory-motor or motivational deficits, may not be sensitive enough to detect small but significant changes in visual acuity. This particularly becomes a problem for the studies of aging that utilize the Morris water task in particular and other vision-mediated behavioral tasks alike.

In examinig aging and memory in 25-27-month-old albino rats, performance on a Morris water task was found to be dependent on the structural integrity of the 
retina (Osteen et al., 1995), suggesting that it is essential to consider vision during design of experimental procedures, both in aging and in general. Despite the considerations, the visual acuity in aged rats has not been assessed in a systematic manner yet.

Given the highest acuity threshold, FBNF1 rats make a good choice for experiments employing vision-mediated behavioral tasks. FBNF1 rats are also bred and maintained by the National Institute on Aging (NIA, Bethesda, MD) specifically for gerontology research purposes. The question remains, however; is the visual acuity of this strain affected by age? In order to address this question, we employed two different tasks. In one experiment we measured the grating acuity in 14 month and 26 month old rats, and in the other we employed the virtual optomotor system to quantify visual acuity of 4,14 , and 26 months old rats.

\subsection{Materials and Methods}

Animals, Six middle-aged (14 months old) and six old (26 months old) male Fisher $344 \mathrm{X}$ Brown Norway rats (NIA/Harlan colonies) were tested on the Visual Water Task. Six young (4 months old), six middle-aged (14 months old), and six old (26 months old) female Fisher $344 \times$ Brown Norway rats (NIA/Harlan colonies) were tested on the virtual optomotor system. All rats were free of cataracts. Animals were cared for and tested at The University of Lethbridge 
vivarium according the National Institutes of Health guidelines and under the approval of the institutional animal care committee which only approves studies that are in accordance with the Canadian Council on Animal Care guidelines. All animals were housed in pairs in hanging plastic cages, in a room with an ambient temperature of $21^{\circ} \mathrm{C}$ and $35 \%$ relative humidity, $12 / 12 /$ light dark cycle. Food and water were available ad libitum.

The Visual Water Task. The Visual Water Task (VWT) has been previously described in detail by Prusky et al. (2000a). Briefly, a trapezoidal metal tank $(140 \mathrm{~cm} \mathrm{~L} \times 80 \mathrm{~cm} \mathrm{~W} \times 25 \mathrm{~cm} \mathrm{~W} \times 55 \mathrm{~cm} \mathrm{H})$ was placed on a solid table and filled with tap water to a depth of $15 \mathrm{~cm}$. The wider end wall of the tank was transparent. A midline divider $(40 \mathrm{~cm} \mathrm{H})$ was placed in the tank and extended $46 \mathrm{~cm}$ into the pool from the transparent wall creating the two arms at the end of which the two computer monitors (17" VGA; Viewsonic E7OF) were positioned on the outside of the tank (Figure 1). The bottoms of the screen monitors were level with the water surface. The monitors were used to control the presentation of stimuli. An Apple Macintosh computer (Power PC G4, $400 \mathrm{mHz}$ ) and a computer program (Vista; http://www.cerebralmechanics.com) were used to control the stimulus presentation, right/left randomization pattern, record behavioral responses, and provide parameter control. A transparent Plexiglas platform ( $37 \mathrm{~cm} \mathrm{~L} \times 13 \mathrm{~cm} \mathrm{W \times 14} \mathrm{cm} \mathrm{H}$ ) was submerged at the end of one of the arms, and always paired with the correct ( + ) stimulus. 
The VWT task capitalizes on rat's natural inclination to escape water by finding a solid substrate (a platform in this case). The rats were first pre-trained to associate the escape from water with the platform by learning to discriminate between the low spatial frequency grating from a gray screen, spatial frequency always being paired with the platform according to the procedure outlined by Prusky et al. (2000a). A low spatial frequency, vertical sine wave grating (0.12 cpd; 4 sine wave cycles on the computer screen), was displayed on one monitor, and if chosen rewarded $(+)$ by escaping the water. A uniform gray of the same mean luminance (- stimulus) was displayed on the other monitor. The escape platform was located directly below the rewarded stimulus. On each trial, rats were removed from their holding cage and released into the pool facing the computer screens. Once on the platform, they were allowed to remain there for a few seconds before being returned to their holding cages. On the next trial the location of the grating and the platform were switched to the opposite arm of the pool, and the side of presentation alternated from then on.

During the training phase, the rats were trained to discriminate between the same two stimuli as in the pre-training phase, the sine wave grating versus gray screen. Once the rats performed at $80 \%$ correct on 30 consequent trials the testing of grating threshold was initiated employing a method-of-limits procedure where incremental changes in the spatial frequency of the sine wave stimulus were implemented. More concretely, if a rat made a correct choice one sine wave cycle was added to the grating $(\sim 0.3-0.49 \mathrm{cpd})$ on the subsequent trial. This continued until the performance accuracy fell below $70 \%$. 
If an error occurred, additional trials with the same spatial frequency were administered until three correct choices were made in sequence, or seven correct choices out of 10 consecutive trials after which the sine wave cycle of the gratings was increased on the next trial. As the spatial frequency increased ( $0.5-0.8 \mathrm{cpd}$ ), the minimal number of trials at each spatial frequency to allow the addition of one sine wave cycle was increased to three. A similar error correction procedure as described above was used; either five consecutive correct trials or seven out of 10 trials were required before spatial frequency could be increased. In the next block of spatial frequencies ( $\sim 0.8-1.0 \mathrm{cpd})$, five consecutive correct trials or seven out of 10 trials were needed to warrant an increase in spatial frequency. For the gratings with spatial frequencies higher than $\sim 1.0 \mathrm{cpd}$, seven out of 10 correct trials were required to proceed with an increase in spatial frequency. The choice was deemed correct if the rat swam to the platform without entering the arm displaying the non-rewarded stimulus. The rat was judged as entering the incorrect arm if the hind legs crossed into this arm of the pool, even if the rat had turned around before reaching the platform. The pattern of the rewarded stimulus/platform location was a left (L), right $(R)$, LLRLRR sequence. The rats swam in a group of six, one at a time, and the inter-trial interval was approximately four minutes.

A preliminary grating threshold was established when animals failed to achieve $70 \%$ accuracy at a specific spatial frequency. To confirm the validity of the threshold the spatial frequency of the grating was decreased by $3-4$ cycles $(0.9$ 
$-1.2 \mathrm{cpd}$ ) and the experimental procedure described above was followed until the performance again dropped below $70 \%$. The point at which the performance repeatedly dropped below $70 \%$ accuracy was recorded as the grating acuity.

The Virtual Optomotor System. A virtual cylinder comprised of a vertical sine wave grating was projected in $3 D$ coordinate space on computer monitors arranged in a square around the testing arena. The arena consisted of a Plexiglas box $(39 \mathrm{~cm} \mathrm{~L} \times 39 \mathrm{~cm} \mathrm{~W} \times 32.5 \mathrm{~cm} \mathrm{H}$ ) with rectangular openings ( 33.5 $\mathrm{cm} \mathrm{W} \times 26.5 \mathrm{~cm} \mathrm{H}$ ) on each wall painted white on the inside. A mirror with a small hole in the center of it was positioned tangentially to the bottom of the openings. A vented lid enclosed the top of the apparatus. A FireWire camera (iSight; Apple Computer Corporation) was attached to the lid with a holding sleeve and centered over the apparatus.

A computer program (OptoMotry@; Cerebral Mechanics), running on a dualprocessor G4 Power Macintosh (Apple Computer Corporation), was used to drive Open GL-compatible video cards (Radeon 7000 Mac Edition; ATI) and project on the monitors a virtual cylinder in 3D coordinate space. Visual stimuli were presented on the walls of the cylinder and the image was reflected by the floor and ceiling mirrors such that each monitor appeared as a window onto the surrounding 3D world. The program controlled the speed of rotation and geometry of the cylinder, the spatial frequency and contrast of stimuli, and enabled live video feedback of the testing area. 
Rats were placed on the platform in the center of the apparatus one at a time and the lid was closed. As the rat moved freely around the platform, the experimenter followed the rat's head with a crosshair superimposed on the video image. The crosshair's $X, Y$ coordinates were used to center the rotation of the cylinder at the rat's viewing position so that the virtual walls of the cylinder were maintained at a constant distance from the animal. When a grating detectable by the rat was projected on the cylinder wall and the cylinder was rotated (12 $\mathrm{deg} / \mathrm{sec}$ ), the rat would stop moving and would begin to track the grating with reflexive head movements.

The experimenter assessed tracking by monitoring the video image of the cylinder, the animal, and the crosshair simultaneously. If the rat's head tracked the cylinder rotation (moved against stationary arms of the crosshair), it was judged that the animal could see the grating. Again, the staircase or method-oflimits procedure was used to systematically increase the spatial frequency of the grating until the rat no longer responded. The highest spatial frequency that the rat could track in either clockwise or counterclockwise direction was recorder as the threshold. The apparatus and the training procedures are described in more detail elsewhere (Prusky et al., 2004). 


\subsection{Results}

The Visual Water Task. The effect of age on grating acuity was statistically assessed by performing a one-way univariate analysis of variance (ANOVA) with the grating acuity as a dependent variable and Group (Middle-aged vs. Old) as an independent variable. The mean grating acuity for the Middle-aged FBNF1 rats was $1.543 \mathrm{cpd}$ and for the old ones $1.36 \mathrm{cpd}$, and this difference in acuity between the two groups was significant $(F(1,11)=77.207, p<.001$; Figure 2).

The Virtual Optomotor System. The visual acuity, as measured by a virtual optomotor system, was statistically assessed in relation to age group by performing a one-way ANOVA. A score for tracking the gratings in clockwise and counterclockwise direction respectively served as a dependent variable and Group (Young, Middle-aged, and Old) served as an independent variable. Although subtle, the difference in tracking in both clockwise $(F(2,17)=5.529, p$ $=.016 ;$ Figure $3 \mathrm{~A})$ and counterclockwise $(F(2,17)=8.107, p=.004$; Figure 3B) direction was significant between age groups. Follow-up tests revealed no difference in acuity between Young and Middle-aged groups ( $p^{\prime} s>12$ ). The acuity of the Old group, however, was significantly lower than the acuity of the Young ( $p$ 's <.015) or the Middle-aged ( $p$ 's <.018) groups. 


\subsection{Discussion}

The main goal of the present study was to assess whether age has an effect on visual acuity of the FBNF1 rats. We found that the acuity, as measured by the grating acuity test did drop from $\sim 1.54 \mathrm{cpd}$ in the middle-aged rats to $\sim 1.36$ cpd in the old rats. Our results suggest that this difference in acuity was significant. Similar results were observed with the virtual optomotor system, where there were no differences in acuity between the Young and Middle-aged rats, but the grating threshold of the Old group was significantly lower from that of the other two age groups.

Our results also replicate findings reported by Prusky et al. (2002), that the visual acuity of the FBNF1 strain of rats clusters at about $\sim 1.5 \mathrm{cpd}$. The rats in the present study with visual acuity of $\sim 1.5 \mathrm{cpd}$, however, were 14 months of age while the rats tested by Prusky et al., (2002) were under 6 months of age. By comparing the present findings pertaining to the visual acuity of the FBNF1 strain of rats to those reported by Prusky et al. (2002) we can draw two additional conclusions: (1) that there are no changes in visual acuity up to the 14 months of age in this strain of rat, and (2) that the drop in acuity occurs somewhere between 14 month and 2 years of age.

Notwithstanding the significant difference in the grating acuity, it is important to note that the acuity of old FBNF1 rats (age $>24$ months; $1.36 \mathrm{cpd}$ ) is still much better than the acuity of young adult pigmented rats (such as the Long-Evans 
rat for example), which clustered around $1.0 \mathrm{cpd}$ (Prusky et al., 2002). Similarly, the acuity threshold of the old FBNF1 rats, as measured by the virtual optomotor system, is not significantly different from the acuity of the young Long-Evans rats ( .54 cpd; unpublished observations). Collectively, our results suggest that the Fisher $344 \times$ Brown Norway hybrid rats are a suitable strain for aging research employing vision-mediated behavioral tasks. 
7. Experiment 2 - Long -Term Potentiation

Is hippocampal synaptic plasticity affected by age? - A study of long-term potentiation

\subsection{Background}

In the early 1970's, Bliss and colleagues (Bliss \& Lomo, 1973; Bliss and Gardner-Medwin, 1973) reported a long-lasting enhancement of synaptic strength in response to high-frequency stimulation. This phenomenon came to be commonly known as long-term potentiation (LTP; Douglas and Goddard, 1975) or long-term enhancement (LTE). Early experiments suggested that LTP could be a cause of synaptic changes underlying memory formation (see Rosenzweig and Barnes, 2003 for a review). Research also suggests that agerelated learning and memory impairments are, at least in part, caused by altered hippocampal synaptic plasticity (Barnes, 2003; Barnes \& McNaughton, 1985; Geinisman et al., 1995; Rosenzweig \& Barnes, 2003). Hence, altered hippocampal synaptic plasticity represents the primary electrophysiological correlate of age-related memory impairments.

There is agreement that age does have an effect on LTP in the dentate gyrus of the hippocampus, but only under certain conditions. For example, such conditions, which have been blamed for the variable results reported in the 
literature, include the stimulation parameters and the strain of rat. Whether age-related differences are seen also depends on whether induction, maintenance, or decay of LTP is being measured. Since the pioneering report by Barnes (1979), it has been widely accepted that LTP decays more quickly in the aged rats. In addition, performance on hippocampus dependent tasks was correlated with the decay rate of LTP in both rats (Barnes, 1979; Barnes and MCNaughton, 1980) and mice (Bach et al., 1999). Whether aged rats have a deficit in LTP induction, however, remains a subject of debate.

Many early experiments of the effect of age on LTP, using long, high-frequency stimulation, report no age-related deficits in LTP induction in the dentate gyrus of the hippocampus in vivo (Barnes, 1979; deToledo-Morrell and Morrell, 1985) and in vitro (Diana et al., 1994a,b). There are just as many experiments, however, reporting age-related LTP induction deficits (Lynch and Voss, 1994; McGahon et al., 1997; Murray and Lynch, 1998a,b; Tielen et al., 1983). Given that most of the experiments reporting deficits come from a single laboratory, they have been largely discounted and their findings attributed to crucial methodological differences and possibly the strain of rat used (see a review by Geinisman et al., 1995).

Instead, the view proposed by Barnes and colleagues, that there are no agerelated deficits in the induction of LTP, has dominated this field for the past 20 years. This view is based on the finding that old rats are capable of reaching the same maximum level of enhancement as the young rats, and does not take as 
important the fact that the old rats take twice as long to reach the maximum than do young rats (Barnes and McNaughton, 1985). Recently Barnes et al. (2000) demonstrated, however, an increased threshold for LTP induction at the perforant path/deritate gyrus synapse, suggesting that there is an age-related induction deficit in addition to a deficit in LTP maintenance.

For our current set of experiments, we chose the Fisher $344 \times$ Brown Norway (FBNF1) hybrid rats bred by the NIA particularly for aging research purposes. We now know (from Experiment 1) that these rats have a high visual acuity, especially in young but reasonably so in old age, making them especially suitable subjects for testing learning and memory in vision-mediated behavioral tasks. We decided to evaluate hippocampal synaptic plasticity in the FBNF1 strain using high-frequency stimulation before proceeding with assessment of learning and memory abilities.

Our motivation for assessing synaptic plasticity in FBNF1 rats in relation to age revolved around the fact that the hippocampal synaptic plasticity had not been investigated much in the FBNF1 rats so we did not know what to expect in terms of synaptic plasticity in these animals. In addition, the strain of rat along with stimulation parameters has been seriously considered as a possible cause of disparate findings in the literature on LTP in the aged hippocampus. Hence, the primary goal of the present experiment is to identify whether there are agerelated changes in hippocampal synaptic plasticity in FBNF1 rats, given that we plan to use this strain of rat for further studies on the hippocampus and aging. If hippocampal LTP is compromised in aged FBNF1 rats and it recruits similar 
synaptic plasticity mechanisms as memory formation, then we predict that agerelated LTP deficits should be associated with age-related memory deficits. The memory deficits should be particularly notable on tasks that are dependent upon the integrity of the hippocampus.

\subsection{Materials and Methods}

Animals. Ten middle-aged (12 month old) and ten old ( 24 month old) male FBNF1 rats $(350-425 \mathrm{~g}$ at the start of the experiment) were housed in pairs in clear plastic cages in a colony room at the University of Lethbridge that was maintained on a $12 \mathrm{hr}$ on $/ 12 \mathrm{hr}$ off light cycle. Rats had access to food and water ad libitum. All animals were cared for and tested at the University of Lethbridge vivarium according to the National Institutes of Health guidelines and under the approval of the institutional animal care committee, which only approves studies that are in accordance with the Canadian Council on Animal Care guidelines.

Surqical and Recording Procedures. All rats were implanted unilaterally with a stimulating electrode (Figure $4 \mathrm{~A}$ ) in the perforant path and a recording electrode in the dentate gyrus (Figure $4 B$ ). The rats were anesthetized with isofluorane (induction at $2.5-4 \%$; maintenance at $1-2.5 \%$; in $1.5 \mathrm{~L} / \mathrm{min} \mathrm{O}_{2}$ ) and stereotaxically implanted with a single stimulating and a single recording electrode. The electrodes were constructed using Teflon-coated stainless steel wire, $114 \mu \mathrm{m}$ in diameter crimped onto gold-plated male amphenol pins (A \& M 
Systems).

The recording electrode was lowered into the hilar region of the dentate gyrus (Paxinos and Watson, 1986; coordinates: $3.2 \mathrm{~mm}$ posterior to bregma, $1.6 \mathrm{~mm}$ lateral to the mid-sagittal suture and approximately $3.5 \mathrm{~mm}$ below the dura matter) and the stimulating electrode was lowered into the ipsilateral perforant path (Paxinos and Watson, 1986; coordinates: $8.1 \mathrm{~mm}$ posterior to bregma, 4.3 $\mathrm{mm}$ lateral to the saggital suture, and approximately $3.0 \mathrm{~mm}$ below the dura matter). Three stainless steel jewelers' screws tapped into the skull served as reference and ground components of the differential recording circuit. The position of both the stimulating and recording electrode was optimized under electrophysiological guidance using single pulse stimulation (pulse duration = $100 \mu \mathrm{s}$, amplitude $=300 \mu \mathrm{A}$, frequency $=1$ pulse $/ 30 \mathrm{~s}$ ). The electrodes were secured in place using dental acrylic. For the chronic experiment, the amphenol pins were inserted into a 9-pin McIntyre-Molino plug, which was subsequently cemented onto the skull.

All rats were habituated to the recording room for two 15-minute sessions for two days before recordings began. All recordings sessions were performed in the animal's own home cage. Evoked potentials were recorded from the hippocampus using Data Wave System Experimenter's Workbench Package. A computer was interfaced with a digital stimulator (AMPI Master 8) connected to a constant current stimulator/stimulus isolation unit (AMPI iso-flex) for electrical stimulation. All stimulation pulses were rectangular monophasic pulses, $100 \mu \mathrm{s}$ 
in duration. The evoked potentials were differentially recorded, amplified 200 times, and filtered at half amplitude between $1 \mathrm{~Hz}$ and $10 \mathrm{kHz}$. The signal was acquired by the computer at a sampling rate of $33 \mathrm{kHz}$, triggered 5 ms before and continuing for $30 \mathrm{~ms}$ after the delivery of each stimulation pulse. An audio analogue of the signal was monitored, and all the individual sweeps were displayed on the computer monitor and acquired on the hard drive.

One week after implantation two baseline input-output (I-O) measures were obtained from awake freely-behaving rats. Stimulation pulses were delivered at $100,300,500,700$ and $900 \mu \mathrm{A}$. Chronic LTP was induced using high frequency stimulation (HFS; $20 \mathrm{~ms}$ bursts of $500 \mathrm{~Hz}$ stimuli, repeated 10 times) delivered once per day for 10 consecutive days followed by I-O measures twice daily 1 and 24 hours following HFS.

For each rat, the evoked potentials were analyzed for population spike amplitude and excitatory postsynaptic potential (EPSP) slope, and the resultant values were averaged at each intensity. The population spike served as an index of granule cell excitability, and was measured as the area under the tangent between the spike onset and offset. The EPSP slope served as an index of synaptic strength, and was measured as the slope of a line between the field EPSP onset and the onset of the population spike. These two measures were derived using the Data Wave software program. All values obtained for the follow-up recordings were analyzed as averaged fractional changes from baseline. 


\subsection{Results}

\section{Effects of HFS on population spike amplitude in middle-aged and old}

rats. Repeated measures analyses of variance (ANOVA) was conducted to compare the population spike (PS) amplitude for the middle-aged and old group either 1 hour or 24 hours post high-frequency stimulation (HFS) respectively across 10 consecutive recording days. A repeated measures ANOVA revealed an Age $X$ Day interaction, whereby the HFS led to a differential effect on PS amplitude for middle-aged and old rats 1 hour post-stimulation across the 10 days of recording $(F(1,120)=8.86, p<0.001)$. For each repeated measures ANOVA, follow-up tests compared the middle-aged and old groups on each day. Follow-up tests revealed that whereas the PS amplitude of middle-aged and old rats did not differ 1 hour after the first HFS session, it differed between groups after each subsequent session (all significant comparisons: $p<0.05$; see Figure 5A). A t-test was computed to compare the baseline PS amplitude for the middle-aged and old rats to the PS amplitude obtained 1 hour following the first HFS session and revealed a significant increase in PS amplitude for both middleaged $(t(9)=-4.783, p=.001)$ and old rats $(t(9)=-2.967, p=.016)$. The increase in the PS was evident in the middle-aged rats but not in the old rats following the initial increase after the first session of HFS.

A separate repeated measures ANOVA revealed an Age $X$ Day interaction, whereby the HFS led to a differential effect on PS amplitude for middle-aged and old rats 24 hours post stimulation $(F(1,120)=8.69, p<0.001)$. Follow-up 
tests revealed that whereas the PS amplitude of middle-aged and old rats did not differ 24 hours after the first HFS session $\left(p^{\prime} s>0.05\right)$, it differed between groups after each subsequent session (all $p^{\prime} s<0.05$; see Figure 5B). Again, the increase was evident in the middle-aged but not old rats.

A t-test was computed to compare the baseline PS amplitude to the PS amplitude obtained 24 hours following the first HFS session and revealed a significant increase in PS amplitude for middle-aged $(t(9)=8.683, p<.001)$ but not old rats $(t(9)=1.351, p=.214)$. Representative responses evoked in the dentate gyrus of the hippocampus by stimulation of the ipsilateral perforant path prior to, 1 hour after the first, and 24 hours after the $10^{\text {th }}$ HFS session for the middle-aged and old rats are shown in Figure $6 \mathrm{~A}-\mathrm{H}$.

Effects of HFS on the EPSP slope of middle-aged and old rats. Repeated measures ANOVAs were conducted to compare the excitatory post-synaptic potential (EPSP) slope for the middle-aged and old group either 1 hour or 24 hours post high-frequency stimulation (HFS) respectively across 10 consecutive recording days. For each repeated measure ANOVA, follow-up tests compared the middle-aged and old group on each day. We found an Age X Day interaction, whereby the HFS led to a differential effect on EPSP slope for middle-aged and old rats 1 hour post-stimulation across the 10 days of recording $(F(1,120)=$ $2.124, p=0.023$ ). Follow-up tests revealed that whereas the EPSP slope of middle-aged and old rats did not differ 1 hour after the first HFS session, it differed between groups after each subsequent session ( $p^{\prime} s<0.05$; Figure $5 C$ ). 
Post hoc analyses revealed that a significant increase in EPSP slope occurred 1 hour following the second HFS session in the middle-aged rats, and 1 hour following the $7^{\text {th }}$ HFS session in the old rats $(p<0.05)$.

A t-test was computed to compare the baseline EPSP slope for the middle-aged and old rats to the EPSP slope obtained 1 hour following the first HFS session and revealed that there was no significant increase in EPSP slope in either the middle-aged or the old rats. A t-test was computed to compare the baseline EPSP slope for the middle-aged and old rats to the EPSP slope obtained 1 hour following the first HFS session and revealed no significant increase in both groups ( $p$ 's $>$.25). A separate t-test was computed to compare the baseline EPSP slope for the middle-aged and old rats to the EPSP slope obtained 1 hour following the second HFS session and revealed a significant increase in EPSP slope for middle-aged $(t(9)=2.595, p=.029)$ but not old rats $(t(9)=.583, p$ $=.576)$. The increase in the EPSP was evident in the middle-aged rats but not in the old rats following the initial increase after the first session of HFS.

A separate repeated measures ANOVA revealed an Age $X$ Day interaction, whereby the HFS led to a differential effect on the EPSP slope for middle-aged and old rats 24 hours post stimulation $(F(1,120)=4.92, p<0.001)$. Follow-up tests revealed that whereas the EPSP slope of middle-aged and old rats did not differ 24 hours after the first HFS session, it differed after each subsequent session ( $p^{\prime} s<0.05$; see Figure 5D). A t-test was computed to compare the baseline EPSP slope to the EPSP slope obtained 24 hours following the second 
HFS session and revealed a significant increase in EPSP slope for middle-aged ( $t$ $(9)=3.946, p<.001)$, and a non-significant trend for the old rats $(t(9)=$ $1.896, p=.09)$.

Additionally, a difference score was computed for both the PS amplitude and EPSP slope respectively across 11 recording days (baseline and 10 HFS sessions) in order to obtain a measure of 24 -hour retention between the recordings obtained 1 hour and 24 hours after baseline and each subsequent HFS. The differences in the percentage of change in both PS amplitude and EPSP slope retained for 24-hours after each HFS were largely insignificant in both middleaged and old rats ( $p$ 's $>.07)$. The only significant differences in 24-hour retention were observed for EPSP slope after the second HFS $(p=.017)$, which is also the first day when group differences emerged in the amount of change in the slope of EPSP. Significant differences in 24-hour retention of PS amplitude were observed at baseline $(p=.024)$ and after $4^{\text {th }}$ HFS session $(p=.044)$.

\subsection{Discussion}

Despite the repeated HFS over 10 consecutive days, we find a substantially reduced responsiveness in the hippocampus of the 24-month old FBNF1 rats compared to the 12-month old rats. Even though both groups of animals potentiated to the same degree on the first day of stimulation, the hippocampal 
circuitry of 12-month old FBNF1 rats seems to remain more plastic than that of the 24-month old rats.

There was a significant change in PS amplitude compared to baseline after the very first HFS in both age groups, but not in the EPSP slope. For each following day of HFS, fractional change from baseline in PS amplitude of old rats changed very little from the level achieved after the first HFS. The fractional change from baseline in PS amplitude of middle-aged rats, however, increased significantly after the second HFS session and reached asymptote on day 8 (Figure 5A \& B). Similarly, the fractional change in the EPSP slope increased significantly after the second day of HFS in the middle-aged animals and continued to significantly differ from baseline across the 10 days of stimulation. For the old rats, a significant increase in the EPSP slope occurred following the $7^{\text {th }}$ HFS session. Although the possibility remains that eventually the old rats could potentiate to the same degree as the middle-aged rats, our results suggest that there is a deficit in the induction of LTP in aged FBNF1 rats.

Similarly, the pioneering work by Barnes (1979) suggested that there were no age-related difference in the magnitude of LTP induction after the first day of stimulation. The difference emerged in persistence of LTP, and only after the third day of stimulation suggesting that repeated HFS is needed for sustained LTP even in the young animals. The effect of repeated stimulation on LTP persistence was not observed in the aged animals. Another important finding was that the performance on the circular maze correlated with the amount of 
LTP induced after the third HFS session, providing the first support for the hypothesis that LTP and spatial learning may share similar mechanistic processes (Barnes, 1979). The question remained, however, whether old rats could reach the same amount of potentiation, possibly with more repetition. Barnes and McNaughton (1980) examined this possibility over 12 consecutive days of repeated stimulation and found that the old rats can eventually reach the same magnitude of LTP as the young rats, a finding that has for years been interpreted as the lack of age-related deficits in LTP induction. The young rats, however, reached their maximum level of potentiation on day 5 , whereas the old rats reached the maximum on day 10 - clearly not a negligible difference in the induction.

The discovery that received more attention was related to the finding that LTP in the old animals, even though it reached the same magnitude of potentiation as it did in the young rats, decayed much faster. This suggested that the rate of forgetting may follow a similar pattern. Indeed, this turned out to be the case (Barnes and McNaughton, 1985). At the same time, reports emerged of a significant relationship between LTP and performance on tasks shown to be dependent on the hippocampal integrity, specifically spatial learning and memory (see Geinisman et al., 1985, Rosenzweig and Barnes, 2003 for a review).

Some of the more convincing evidence for the role of LTP in information processing and storage came from studies that involved manipulations that 
affect both memory and LTP in a similar manner. For example, Morris et al., (1986) demonstrated that pharmacological agents that impair spatial memory in rats also block the induction of LTP. On a similar note, it has been demonstrated that agents that improve spatial memory in aged rats also improve the retention of LTP (deToledo-Morrell et al., 1988).

Our results are similar to those reported by Barnes (1979), in that there are no age-related differences in LTP induction after a single LTP session. Nonetheless, with repeated high frequency stimulation protocols, our findings diverge. Barnes and McNaughton (1980) extended their stimulation protocol to 12 days and found that old rats just take longer to reach the same maximum as the young rats. Notwithstanding the slight difference in stimulation parameters and the strain of rats used in the present study compared to those employed by Barnes $(1979,2003)$ and colleagues $(1980,1985)$, our results suggest that old FBNF1 rats did not benefit from repeated stimulation. After the initial incrase in the populations spike amplitude for both groups after the first HFS session, we observed no additional change in the population spike amplitude of old rats across the remaining nine days of HFS. A significant change in EPSP slope was, however, observed after day 7 in old animals suggesting that maybe they could have benefited from additional HFS. Nevertheless, the fact that there was no substantial change in PS amplitude with repeated HFS, and some change in EPSP but only after day 7 leads us to conclude that hippocampal synaptic plasticity is importantly compromised in aged FBNF1 rats. 
The stimulation parameters and the strain differences remain to be reconciled. For the purpose of present experiments, our findings suggest that age-related changes in hippocampal plasticity may be expected, and may further underlie some of the behavioral deficits on hippocampus-dependent tasks seen with age. Hence, hippocampus-dependent learning and memory deficits are anticipated in the aged FBNF1 rats. 


\section{Experiment 3 - Spatial Learning and Memory}

\section{The Morris water task: Does age have an effect on learning and memory}

of spatial hippocampus-dependent information?

\subsection{Background}

Over the years, numerous tasks have been developed to assess spatial learning and memory in rodents (for examples see a review by Geinisman et al., 1995). A task that is perhaps most commonly used to assess spatial learning and memory, and is considered a litmus test of hippocampus-dependent cognition, is the Morris water task (MWT; Morris, 1981). The performance on this task is similarly affected by hippocampal lesions (Gallagher and Holland, 1992; Morris et al., 1982; Sutherland et al., 1982) and old age (Gage et al., 1984, 1988; Pelleymounter et al., 1987; Rapp et al., 1987; van der Staay, 2002).

The deficits seen with old age, while qualitatively similar compared to the deficits produced by lesions, are thought to be quantitatively less severe. Both aged rodents (Gallagher and Pelleymounter, 1988; Rapp et al., 1987) and those with hippocampal damage (Morris et al., 1982; Morris, 1983) are unimpaired in locating a visible platform, suggesting that the impairment is indeed in learning and memory, and not sensori-motor in nature. 
Spatial learning and memory, in general, are affected in aged humans as well (Driscoll et al., 2003a; Geinisman et al., 1995; Moffat and Resnick, 2002; Moffat et al., 2001; Raz, 2000). More specifically, we found an age-related deficit in performance on the virtual Morris water task, while there were no age-related differences in swimming to a visible platform in the virtual environment. In addition, the age-related deficits in learning and memory for spatial information are not only present in the elderly, but already become apparent in the middleage group (Driscoll et al., 2005).

We already know from Experiment 1 that FBNF1 rats, even the aged ones, have a good visual acuity. Hence, the use of visual tests to assess learning and memory here is appropriate, as it will not be biased by age-related changes in vision. In addition, results of Experiment 2 suggest that the hippocampal synaptic plasticity of our aged rats should be compromised. Given that the relationship between synaptic plasticity and memory is often reported, we expect to find compromised learning and/or memory on tasks dependent on the hippocampus. In order to confirm that our rats have an age-related deficit in learning and/or memory for hippocampus-dependent spatial information, we trained them on the working memory variant of the Morris water task in which the platform moved to a new location after every two days. By manipulating the location of the platform in such a manner, we were able to obtain measures of both acquisition and retention of new information. 


\subsection{Materials and Methods}

Animals. Thirty Fisher 344 Brown Norway hybrid female rats (NIA/Harlan) participated in the experiment. Rats were divided into 3 groups: Young ( $N=10$; age 3 months at the start of the experiment), Middle-aged $(N=10 ;$ age 14 months at the start of the experiment), and Old ( $N=9$; age 24 months at the start of the experiment) group. One old rat died during the MR scanning procedures (which were performed prior to behavioral testing) due to preexisting stroke. All rats were free of cataracts. Rats were maintained at the University of Lethbridge vivarium, housed in pairs in hanging plastic cages, in a room with an ambient temperature of $21^{\circ} \mathrm{C}$ and $35 \%$ relative humidity, $12 / 12$ / light dark cycle. Food and water were available ad libitum. All procedures involving animal care and experimentation were carried out in accordance with guidelines provided by the National Institutes of Health and approved by the institutional animal care committees at the University of Lethbridge, University of Calgary, and the National Research Council of Canada, which only approve procedures that are in accordance with the Canadian Council on Animal Care. All behavioral testing procedures were carried out at the University of Lethbridge.

The Morris Water Task. All rats were trained in a modified version of the Morris water task (Morris, 1981) in which the platform moved to a new location every second day according to a random sequence of locations. This version of the task is useful for observing new learning as well as 24-hour retention. For the first two days the platform remained in the same location. On the $3^{\text {rd }}$ day the 
platform moved to a new location for another pair of days and so on until 4 locations were completed ( 8 consecutive days). So all rats had been exposed to the MWT training for 8 days and experienced 4 different platform locations (center of NE, SW, SE, and NW quadrant). All rats were pre-trained on the task for 2 days prior to data acquisition. In addition, after completion of 8 days of hidden platform training, we administered one day of visible platform training.

Rats received 8 trials on each training day and were released twice from each cardinal compass point around the perimeter of the pool in a pseudorandom sequence. A maximum of $60 \mathrm{~s}$ was allowed to locate the platform. If a rat failed to locate the platform within $60 \mathrm{~s}$, it was removed from the water and placed on the platform for $10 \mathrm{~s}$ before returning to the holding cage.

The apparatus involved a circular pool with white interior, filled to within $20 \mathrm{~cm}$ of the top of the wall with water $\left(21^{\circ} \mathrm{C}, 40 \mathrm{~cm}\right.$ deep $)$ rendered opaque with milk powder. The platform was constructed of clear Plexiglas $(13 \mathrm{~cm}$ diameter), and its top surface was $1.5 \mathrm{~cm}$ below the surface of the water, positioned in the center of the NE quadrant of the pool. Performance was recorded and analyzed by a video tracking system (HVS 2020 Plus system). Measures recorded included latency and path length to reach the platform, and the speed of swimming. 


\subsection{Results}

Latency, path length, and speed of swimming measures were averaged for each trial across all odd (new platform location) and all even (new platform location) days separately, and served as a dependent variable respectively. Repeated measures analysis of variance (ANOVA) was conducted with Trial (8) as a within-subjects variable, and Group (3; Young, Middle-aged, and Old) as a between-subjects variable for each of the dependent measures on odd versus even days.

\section{MWT - Hidden Platform}

Latency. For latency to locate the platform on days (odd) when the platform moved to a new location, we found a significant effect of Trial $(F(7,175)=$ $55.729, p<.001)$ and Group $(F(2,25)=18.532, p<.001)$. The Group X Trial interaction was not significant $(p>1)$. For days (even) when the platform remained in the same location as the previous day, there was a significant Group X Trial interaction $(F(14,175)=3.583, p<.001)$. The effects of Trial $(F$ $(7,175)=18.537, p<.001)$ and $\operatorname{Group}(F(2,24)=18.541, p<.001)$ were also significant. Latency to locate the platform across trials for odd and even days respectively is represented in Figure 7A \& B. Overall, latency across trials as a measure of learning the new platform location suggests that all rats, regardless of their age, were able to acquire the information in a similar manner. 
Path Length. For path length traveled to reach the platform on days (odd) when the platform moved to a new location there was a significant Group X Trial interaction $(F(14,175)=1.848, p<.035)$, and a significant effect of Trial $(F$ $(7,175)=45.747, p<.001)$ and $\operatorname{Group}(F(2,25)=8.075, p=.002)$. For days (even) when the platform remained in the same location as the previous day, there was a significant Group X Trial interaction $(F(14,175)=2.514, p=$ $.003)$. The effects of Trial $(F(7,175)=13.552, p<.001)$ and $\operatorname{Group}(F(2,25)$ $=9.591, p=.001$ ) were also significant. Path length to reach the platform across trials for odd and even days respectively is represented in Figure 7C \& D.As it was the case with latency, path length across trials as a measure of learning the new platform location suggests that all rats, regardless of their age, were able to acquire the information in a similar manner.

Swim speed. For the speed of swimming, on days (odd) when the platform moved to a new location, there was a significant Group $X$ Trial interaction ( $F$ $(14,175)=2.037, p=.018)$. Also, there was a significant effect of Trial $(F(7$, $175)=2.477, p=.019)$, and a non-significant trend for the Group $(p>.05)$. For days (even) when the platform remained in the same location as the previous day, there was a significant Group $\times$ Trial interaction $(F(14,175)=$ $3.957, p<.001)$. The effects of Trial $(F(7,175)=12.747, p<.001)$ and Group $(F(2,25)=10.826, p<.001)$ were also significant. Swimming speed across trials for odd and even days respectively is represented in Figure 7E \& F. Overall, the results suggest that even though significant differences in the speed 
of swimming can be observed on certain trials between groups, the old rats can reach the same maximum speeds as their younger counterparts.

24-hour Retention. In order to assess the retention of newly learned platform location over a period of 24 hours in different age-groups, we computed difference scores between the last trial of the day when the platform was in a new location and the first trial of the next day when the platform was in the same location as the day before. The 24 -hour difference scores were averaged across 4 different platform locations. This measure served as a dependent variable with Group membership as an independent variable. We found a significant difference in 24-hour retention based on age-group membership latency to locate the platform $(F(2,27)=3.583, p=.043$; Figure 8$)$ and a non-significant trend for path length $(p=.082)$, such that both middle-aged and old rats were impaired in 24-hour retention of spatial information.

MWT-Visible Platform, Latency, path length, and speed of swimming served as a dependent variable respectively. Repeated measures ANOVA was conducted with Trial (8) as a within-subjects variable, and Group (3; Young, Middle-aged, and Old) as a between-subjects variable for each of the dependent measures. For latency, there was a significant Trial $X$ Group interaction $(F(14,175)=$ $5.362, p<.001)$, and a significant effect of Trial $(F(7,175)=34.049, p<$ $.001)$ and Group $(F(2,25)=7.624, p=.003)$. Similarly, there was a significant Trial X Group interaction $(F(14,175)=6.008, p<.001)$, and a significant effect of Trial $(F(7,175)=33.006, p<.001)$ and $\operatorname{Group}(F(2,25)=11.660, p$ 
$<.001)$. For the path length to reach the visible platform, there was a significant Trial $X$ Group interaction $(F(14,175)=3.326, p<.001)$, and a significant effect of Trial $(F(7,175)=26.865, p<.001)$, but the effect of Group $(F(2,25)=2.240, p=.127)$ was not significant. Overall, all rats were able to reach the visible platform in a similar manner, regardless of their age. The latency, path length, and speed of swimming to a visible platform across trials are presented in Figure 9.

\subsection{Discussion}

Our results suggest that all animals, regardless of age, were able to learn the platform location. The acquisition of spatial information was more similar for the young and middle-aged rats. The aged rats were able to learn about the platform location to an equal extent by the end of the day on which the platform appeared in a location not previously encountered.

The deficit appeared in 24-hour retention of this newly acquired spatial information for not only the aged but also the middle-aged rats. Old and middleaged rats had much longer latencies and path lengths to reach the platform on the first trial of the day on which the platform remained in the same location as the previous day compared to their performance on the last trial of the previous day. By the end of the day where platform remained in a location in which it appeared the previous day, however, all rats were again performing similarly. 
A similar pattern of behavior was observed for both measures of place learning, latency (Figure 7A \& B) and path length (Figure 7C\& D). Latency is thought to be dependent on the speed of swimming, which most believe changes over the lifespan of a rat, and hence is considered a biased measure of learning and memory in old age. Path length, on the other hand, is a measure independent of swim speed, and hence considered unbiased. We observe similar performance with both measures. Also, while not always swimming at the same speed as their younger counterparts, the old rats are able to do so on most trials (Figure $7 E \& F)$. Together, our results suggest that the memory deficit observed in 24hour retention of newly learned spatial information is not biased by differences in swim speed.

In addition, we allowed all rats to swim to a visible platform for a day (Figure 9). The old rats seemed to have higher latencies at first, but their performance shortly became almost identical to that of middle-aged and young rats. The speed of swimming was more variable than expected, but again the speed was similar for all three groups on certain trials suggesting that the old rats can swim at the same speed as the young rats. As far as path length to locate the platform is considered, all three groups performed similarly. Again, our visible platform results suggest that the observed hippocampus-dependent deficit in retention of newly learned spatial information in relation to age was independent of sensori-motor or motivational biases. 
We have established that the age-related deficit in spatial cognition associated specifically with the hippocampus is present in our rats. Our findings of dysfunctional memory for spatial information add to the extensive literature reporting age-related deficits on spatial tests of learning and memory (see Geinisman et al., 1995; van der Staay, 2002 for a review). What is more interesting, however, is the fact that the pattern of age-related memory deficits for spatial information in the rat is very similar to that reported for human place learning in the virtual Morris water task (Driscoll et al., 2005), in that the deficits are present in both old and middle-aged group. The next question we ask is whether the age-related deficit in learning and memory is present for other types of hippocampus-dependent information processing, or is it specific to spatial information? 


\section{Experiment 4 - The Transverse Patterning Problem}

Does the transverse patterning discrimination problem, as opposed to elemental discriminations, depend on the hippocampus?

\subsection{Background}

Many theorists have suggested that the pattern of spared and impaired performance associated with damage to the hippocampus can be explained by distinguishing between a configural/conjunctive memory system and an elemental or habit system (Hirsh, 1974; Mishkin et al., 1984; O'Reilly \& Rudy, 2001; Sutherland \& Rudy, 1989; Tyler \& DiScenna, 1986; Wicklegren, 1979). Central to these theories is that the hippocampus binds together the independent features of an episode into a unique representation called a configural or conjunctive representation.

Based on their configural association theory of the hippocampal function, Sutherland and Rudy (1989) predicted that non-linear discrimination problems should be especially dependent on the hippocampus. Problems in this category do not permit solutions based on the associative strengths of the individual elements. One such problem is called transverse patterning (Alvarado \& Rudy, 
1992, 1995a,b; Spence, 1952). The transverse problem involves three problems comprised of only three stimuli, so that each stimulus participates in two out of the three problems comprising this task and each stimulus is equally ambiguous; $A+B-, B+C-, C+A-$. The transverse patterning problem requires attending to the relationships between the stimuli and is said to require the hippocampus, as learning about each stimulus separately will not lead to a successful solution to this problem. In contrast, elemental discriminations, while similarly involving three problems, are comprised of six unambiguous stimuli; $A+B-, C+D-; E+F-$. Successful solution to this problem can be obtained by simply learning that certain stimuli are either always rewarded or non-rewarded. Elemental discriminations can be solved without the intact hippocampus.

There have been several reports of anterograde amnesia for the transverse patterning problem after the damage to the hippocampus (Alvarado \& Rudy, 1995a,b; Dusek \& Eichenbaum, 1998; but see Bussey et al., 1998). For example, Alvarado and Rudy (1995a) fourd that rats with damage to the hippocampus could not solve the transverse patterning problem, but they readily learned to solve a control set of three elemental discrimination problems. Such results are consistent with the idea that the hippocampus makes a selective contribution to problems that require configural/conjunctive solutions. The finding that damage did not impair learning the set of elemental discriminations suggests that there is a system that does not depend on the hippocampus that can support a solution to these problems. 
Even though rats with damage to the hippocampus are not impaired in learning elemental discriminations, these data do not speak to a fundamental question; does the hippocampus contribute to the learning of these elemental problems in the normal, intact subject? The answer to this question is important because behavioral tests following anterograde damage to the hippocampus only reveal the residual capacities afforded by spared, extra-hippocampal circuitry. They do not necessarily reveal the contribution that the hippocampus makes when it is intact in a normal animal. Thus, the fact that rats with damage to the hippocampus are not impaired on the set of elemental discrimination problems does not mean that the hippocampus makes no contribution to solving these problems in the intact animal.

We are interested in using the transverse patterning discrimination task as a non-spatial test of hippocampal integrity in aged rats. Before we do so, we decided to test definitively whether this task, as implemented in our laboratory engages the hippocampus or not. The purpose of the present study is twofold. First, we attempt to resolve the discrepancy between the studies reporting a spared performance on the transverse patterning problem after hippocampal damage and those reporting impairments. The secondary goal is to determine if the hippocampus contributes at all to learning elemental discriminations. To implement the experimental design, we used a water escape task (Prusky et al., 
2000a) to control the presentation of stimuli in the visual discrimination problems.

\subsection{Materials and Methods}

Animals. Subjects were 8 female Brown Norway $x$ Fisher 344 (BNxF344) hybrid rats (Harlan, IN), $12-14$ months of age, $250-325 \mathrm{~g}$. Four rats were assigned to the elemental discrimination condition, and four rats were assigned to the transverse patterning condition. One rat in the elemental discrimination group died due to respiratory infection and did not complete the last two conditions of training (the 10-day delay test after relearning the elemental discriminations and learning a new set of problems post-surgery). The rats were group-housed in hanging plastic cages in a room with an ambient temperature of $21^{\circ} \mathrm{C}, 35 \%$ relative humidity, $12 / 12$ light/dark cycle, and had access to food and water ad libitum. All experiments were carried out in accordance with the guidelines provided by the Canadian Council on Animal Care.

Surgery. The hippocampal formation was damaged by bilateral injections of NMDA (10 $\mathrm{mg} / \mathrm{ml})$ dissolved in phosphate-buffered saline. Rats were anesthetized by isofluorane inhalation ( $4 \%$ with $2 \mathrm{l} /$ minute of oxygen and $2 \%$ after a surgical plane was established). A midline incision was made in the scalp and periosteum. Rats received 5 injections in each hippocampus through a 30gauge cannulae; at the rate of $0.1 \mu \mathrm{l} / \mathrm{min}$ using an infusion pump, a $0.4 \mu \mathrm{l}$ of 
excitotoxin at each site $(3.1,4.1,5.0,5.3$, and 6.0 posterior to bregma; 1.5 , $3.0,3.0,5.2$, and $5.0 \mathrm{~mm}$ lateral to bregma; and 3.6,4.0, 4.0, 7.3, and $7.3 \mathrm{~mm}$ ventral to the surface of the skull) in respective order. Cannulae were left in place for 4 min after each injection. The scalp was sutured and the animal was returned to its home cage to recover. Recovery was monitored for 90 minutes before the animal was returned to the colony room. Animals were administered clordiazepoxide ( $1 \mathrm{mg} / \mathrm{kg}$, i.p.) 20 minutes before surgery, and then on the first sign of wakefulness after surgery for 3 post-surgery injections total, approximately 30 min apart to suppress any seizure activity that may be associated with NMDA injections. Animals were allowed to recover for 10 days before the testing began.

Histology. All animals were sacrificed by overdose of pentobarbital (100 mg/kg i.p.) and perfused transcardially with $0.9 \%$ saline followed by $4 \%$ paraformaldehyde. The brains were removed and $40 \mu \mathrm{m}$ coronal sections were cut on a cryostat microtome. Every fifth slice was mounted on glass slides and stained with cresyl violet. The stained sections were examined under a microscope to quantify damage.

\section{Discrimination Training Procedures}

Visual Water Task. The Visual Water Task (VWT) was used to train rats on the discrimination problems. Prusky et al. (2000) previously described this task in detail. Briefly, a trapezoidal metal tank $(140 \mathrm{~cm} \mathrm{~L} \times 80 \mathrm{~cm} \mathrm{~W} \times 25 \mathrm{~cm} \mathrm{~W}$ and 55 
$\mathrm{cm} \mathrm{H}$ ) was filled with water to a depth of $15 \mathrm{~cm}$. The end wall of the tank was transparent. A midline divider $(40 \mathrm{~cm} \mathrm{H})$ was placed in the tank and extended $46 \mathrm{~cm}$ into the pool from the transparent wall. This divider separated two computer monitors (17" VGA; Viewsonic E7OF) that were positioned outside the transparent wall of the pool and used to present the stimuli. A transparent Plexiglas platform $(37 \mathrm{~cm} \mathrm{~L} \times 13 \mathrm{~cm} \mathrm{~W} \times 14 \mathrm{~cm} \mathrm{H}$ ) was submerged at the end of one of the arms, and always paired with the correct (+) stimulus. A computer program (Vista; http://www.cerebralmechanics.com) was used to control the right/left randomization pattern, present discriminanda, record behavioral responses, and provide parameter control. The discriminanda are shown in Figure 10.

Overview. Rats were shaped to associate swimming to the platform with escape from the water. The platform was located directly below the monitor displaying the rewarded $(+)$ stimulus. At the beginning of each trial, animals were released into the pool facing the transparent wall, and allowed to swim to one of the stimuli. If choice was correct, rat was allowed to climb the platform and remain there for a few seconds before being returned to the holding cage. If incorrect, the rat was trapped in that arm of the pool for $10 \mathrm{~s}$ and then allowed to swim to the arm containing the platform before being removed from the pool. Trapping was not instituted on the test day. The choice was correct if animals swam to the platform without entering the arm displaying the non-rewarded stimulus. The rat was judged as entering the incorrect arm if the hind legs crossed into this arm of the pool, even if the rat had turned around before 
reaching the platform. The pattern of the rewarded stimulus/platform location was a left $(L)$, right $(R)$, LLRLRR sequence. The dependent variable was percentage of correct choices. The intertrial interval was approximately three minutes.

The overall design of the experiment is presented in Table 1. All rats were initially trained on their respective set of discrimination problems, as just described. Following the test day, each rat's hippocampus was damaged bilaterally and 10 day later they were retested. Each rat was then retrained and tested using the same procedures described above. The rats trained on the elemental problem set were then trained on a new set of elemental discrimination problems (Figure 10, Set $2: \mathrm{G}+\mathrm{H}-, \mathrm{I}+\mathrm{J}-, \mathrm{K}+\mathrm{L}-$ ).

Elemental Discriminations, Rats were trained to solve three visual discriminations (Figure 10, Set 1: $A+B-, C+D-, E+F_{-}$) using a stepwise approach. Training consisted of nine days in three phases ( 3 days for each phase, ), a test on day 10 , followed by a novel transfer testing on Day 11 . Phase 1 consisted of 3 days of training on Problem 1, 30 trials each. In Phase 2, Problem 2 was introduced and intermixed with Problem 1. Days 4 and 5 consisted of 5 trials of Problem 1, followed by 20 trials of Problem 2, and again by 5 trials of Problem 1 . On Day 6 Problem 1 and 2 alternated every 5 trials for a total of 30 trials, starting with Problem 1. In Phase 3, Problem 3 was introduced and intermixed with Problems 1 and 2. On Day 7 rats received 5 trials of Problem 1, 20 trials of Problem 3, and 5 trials of Problem 2. Day 8 
consisted of 5 trials of Problem 2, 20 trials of Problem 3, and 5 trials of Problem 1. Day 9 consisted of alternating blocks of 5 trials of each problem $(1,2$, and 3 ). The test day, Day 10, consisted of randomly alternating problems on each trial $(3,2,1,2,1,3,2,3,1,3,1,2,1,2,3 \ldots)$, for a total of 30 trials, 10 of each problem.

Transverse Patterning. Transverse patterning (Figure 10, Set 3: $X+Y-; Y+Z-;$ $Z+X-)$ training consisted of 7 days, followed by a test on Day 8,30 trials per day. Phase 1 consisted of 1 day of training on Problem $1(X+Y-)$. In Phase 2, Problem $2(Y+Z-)$ was introduced and intermixed with Problem 1 for 1 day, and consisted of 5 trials of Problem 1, followed by 20 trials of Problem 2, and again by 5 trials of Problem 1. On Day 3 Problem 1 and 2 alternated every 5 trials for a total of 30 trials, starting with Problem 1. In Phase 3, Problem $3(Z+X-)$ was introduced and intermixed with Problems 1 and 2 . On Day 4 rats received 5 trials of Problem 1, 20 trials of Problem 3, and 5 trials of Problem 2. Day 5 consisted of 5 trials of Problem 2, 20 trials of Problem 3, and 5 trials of Problem 1. Day 6 consisted of alternating blocks of 5 trials of each problem $(1,2$, and 3 ). The test day, Day 8 , consisted of randomly alternating problems on each trial $(3,2,1,2,1,3,2,3,1,3,1,2,1,2,3 \ldots)$, for a total of 30 trials, 10 of each problem. 


\subsection{Results}

Histology. The damage to the hippocampal formation was extensive bilaterally (Figure 11 ). On average, approximately $70 \%$ of the hippocampus was removed. The smallest lesion encompassed $50 \%$ of the hippocampal formation and the largest lesion involved $95 \%$ of the hippocampus. Three out of seven rats had $50 \%-60 \%$ of the hippocampus damaged, while the remaining four rats had lesion that encompassed $85 \%$ or more of the hippocampus. Sparing of the major hippocampal subfields was limited almost exclusively to the postero-ventral regions. The damage did not extend to the thalamus, subicular region, or rhinal cortices.

Elemental Discriminations. We performed a repeated measures analysis of variance with Problem (3) and Test Time (5 levels: pre-lesion, post-lesion retesting, post-lesion re-training, post-lesion re-training with a 10-day delay, and the new discrimination learning) as within-subjects variables, and performance (\% correct) on each problem as a dependent variable. There was a significant Problem $X$ Test Time interaction $(F(8,16)=8.221, p<.001)$. By the completion of training rats were performing at a near perfect level on all three problems. Damage to the hippocampus, however, significantly impaired performance on these problems. Nevertheless, these rats were able to relearn the same set of problems and displayed perfect retention 10 days later (to mimic the recovery period after surgery). They were also able to learn a new set 
of elemental discrimination problems. Figure $12(A \& B)$ presents the results for the various testing phases of the elemental discrimination paradigm.

In order to assess whether the rats learned the problems at a similar rate before and after the damage, we performed a repeated measures ANOVA with Problem (3) and Learning Condition ( 3 levels: pre-lesion, post-lesion re-training, postlesion new problem) as within-subjects variables, and trials to criterion (80\% correct, 8 out of ten consecutive trials) as a dependent variable. There was a significant Learning Condition $X$ Problem interaction $(F(4,8)=5.718, p=$ .018 ), Rats acquired each of the three problems at a faster rate after the lesion regardless of whether the discrimination set was new or encountered prior to lesion (Figure 13), suggesting that the lesion impaired the memory for the stimuli but not the procedural requirements of the task itself.

Transverse Patterning. A repeated measures ANOVA was performed with Problem (3) and Testing ( 3 levels: pre-lesion, post-lesion test, post-lesion retraining) as within-subjects variable, and performance (\% correct) as a dependent variable. There was a significant Learning Condition $X$ Problem interaction $(F(4,12)=3.376, p=.045)$, where intact rats were able to learn the transverse patterning but were unable to remember or relearn the problems after the damage to the hippocampus. Figure 14 presents the results for the transverse patterning testing phases before and after the hippocampus was damaged. Performance on the test day prior to surgery was well above chance on all three problems. Damage to the hippocampus, however, significantly 
impaired performance on each problem learned prior to surgery, and the rats were unable to re-learn them.

\subsection{Discussion}

Damage to the hippocampus produced severe retrograde amnesia for both the transverse patterning and the elemental problems. Equally important, however, was that rats with damage to the hippocampus displayed perfect retention through a lengthy interval following retraining on the elemental problems. Thus, the amnesia they displayed when the hippocampus was damaged following training was not displayed if the problems were learned or relearned in the absence of the hipppocampus. Our results also replicate those of Alvarado \& Rudy $(1995 a, b)$ that rats with damage to the hippocampus are impaired in learning the transverse patterning problem, but can learn a set of elemental problems.

That the damage to the hippocampus severely impaired performance on the transverse patterning problem in both retrograde and anterograde direction is consistent with the theoretical claim that the hippocampus makes a critical contribution to the acquisition of configural representations (Sutherland \& Rudy, 1989). Our results also suggest, however, that the need for configural solution is not a requirement for a problem to engage the hippocampus. The finding that the damage to the hippocampus produced severe retrograde amnesia for the 
elemental problems implies that when the hippocampus is intact it also makes an essential contribution to the acquisition of discrimination problems that can be solved simply on the basis of the associative strengths of the individual elements. We are not alone in making this observation. There have been a number of reports, that hippocampal damage can produce retrograde amnesia for non-configural information even though the same information is spared in anterograde direction (Ross et al., 1984; Sara, 1981; Sutherland et al., 2001; Weisend et al., 1996).

How does the hippocampus contribute to the acquisition of elemental discrimination problems? To approach this question, it should be emphasized that rats can acquire and retain the solution to elemental discriminations problems when the hippocampus is damaged prior to training (Alvarado \& Rudy, 1995a, b). Thus, as has often been proposed (Hirsh, 1974; Mishkin et al., 1984; Sutherland \& Rudy, 1989), there is an elemental association system that can solve problems simply on the basis of the associative strengths of the elements comprising the problems and that does not depend on the hippocampus. Once this is accepted, it then must be the case that if the hippocampus is functioning during training, the independent contribution of the elemental system is significantly compromised. If this is not the case then there should be no retrograde amnesia because without a hippocampus rats learn and retain the solution to these problems. This implies that (a) there is competition between the hippocampal-dependent memory system and the system that does not depend on the hippocampus and (b) that hippocampal system dominates. 
Hence, one contribution of the hippocampus, in addition to its role in storing specific memories, is to reduce the contribution of the elemental system.

It must also be the case that the hippocampus supports a memory representation that is at least partly independent of the elemental system. We noted that a common assumption is that the hippocampus binds together representations of co-occurring events (e.g., Fanselow, 1999, 2000; O'Reilly \& Rudy, 2001, Rudy \& O'Reilly, 2001; Squire, 1992; Sutherland \& Rudy, 1989; Tyler \& DiScenna, 1986). There are at least two ways this mechanism could contribute to solving elemental discriminations. The hippocampus could store conjunctive representations of the individual trial outcomes (e.g. A+ or B-) together with contextual elements that can be used to guide choice behavior. Alternatively, one might assume that when confronted with a set of elemental discrimination problems the intact rat uses the same configural representations it would be forced to use to solve the transverse patterning problem. Alvarado and Rudy (1992), however, reported data that rule out this possibility.

The general conclusions from this analysis are (a) transverse patterning requires the hippocampus to be intact for successful performance, (b) elemental discrimination problems can be solved by a hippocampal-dependent system and a system that does not depend on the hippocampus, and (c) the hippocampaldependent system suppresses or inhibits the extra-hippocampal elemental association system, in such a way that the latter does not develop an independent memory representation sufficient to solve the tasks. 
These theoretical conclusions are remarkably consistent to those derived from recent studies of contextual fear conditioning. If placed into a particular context and shocked rats subsequently display fear of that place or context by freezing. The literature indicates that neurotoxic damage to dorsal hippocampus before conditioning has little or no effect on the acquisition of contextual fear conditioning (Cho et al., 1999; Frankland et al., 1998; Maren et al., 1997; Richmond et al., 1999) but damage to the hippocampus following learning produces severe retrograde amnesia (Anagnostaras et al., 1999; Maren et al., 1997). This dissociation between anterograde and retrograde effects clearly parallels our current conclusions about elemental visual discriminations.

Several theorists (Fanselow, 1999; Maren, 2001; Rudy et al., 2004; Rudy and O'Reilly, 2001) have proposed that that contextual fear conditioning can be supported by a hippocampus-dependent memory system that supports a configural/conjunctive representation of context and an elemental system that supports conditioning to the independent features that make up the context. This idea predicts that the damage to the hippocampus should not impair contextual fear in the anterograde direction because the residual elemental system can support conditioning. To explain why hippocampal damage produces severe retrograde amnesia, Maren and colleagues (1997) made the additional proposal that there is (a) competition for conditioning between the hippocampal conjunctive representation and the feature representations for association with shock, and (b) the conjunctive representation dominates. Thus, 
when the hippocampus is damaged after conditioning the conjunctive representation is no longer available to support fear conditioning and, because conditioning to features was weak, the animal shows amnesia.

It is tempting to conclude that results from two markedly different paradigms, appetitive discrimination learning and contextual fear conditioning, have revealed a common set of principles: (a) in both cases the learning experience potentially can be represented in two systems, one which depends of the hippocampus and one that does not, and (b) in the intact animal the hippocampal system dominates. Rudy et al. (2004), in fact, have recently suggested that these two functions represent two independent contributions of the hippocampus. It stores conjunctive representations and inhibits or interferes with the learning that can be supported by the non-hippocampal system. It is not known from the present data sets how general this interference is across types of learning or whether the inhibitory/interference process is limited to the time of encoding/storage of the memory.

In summary, we asked if the hippocampus makes a contribution to solving a set of elemental discriminations that could be solved simply on the basis of the associative strengths of the individual elements. Our results indicate that the damage to the hippocampus produced severe retrograde amnesia in rats that had solved these problems with hippocampus intact. We propose that this result indicates that the hippocampus provides an independent representation of the solution and inhibits learning that could be supported by a system that does not 
depend on the hippocampus. This conclusion converges with recent theoretical views on the contribution the hippocampus makes to contextual fear conditioning and is consistent with a general process whereby the hippocampus inhibits memory processes in other learning systems.

In addition, we asked if the hippocampus makes a contribution to solving a set of transverse patterning discriminations that require configural solution. Our results indicate that the damage to the hippocampus produced severe retrograde and anterograde amnesia for this set of problems. Hence, the transverse patterning task will serve well as a test of hippocampus-dependent non-spatial learning and memory. The next question we ask is, whether transverse patterning performance is affected by age. 
10. Experiment 5 - Non-spatial Learning and Memory

Does old age have an effect on learning and memory of non-spatial hippocampus-dependent information?

\subsection{Background}

Now that we have resolved that our version of the transverse patterning problem tests hippocampus-dependent learning and memory, we proceed by employing this problem as a test of non-spatial hippocampus-dependent mnemonic function in aging. Previously, this problem has been successfully used to test age-related deficits in hippocampus-dependent cognition in humans (Driscoll et al., 2003a). Conversely, it has never been used to test hippocampusrelated learning and memory in the aged rat. If we presume that preserved integrity of the hippocampus is necessary for successful performance on this task, and our findings from Experiment 4 suggest that it is, and given that the reports of compromised hippocampal integrity with old age are numerous (Geinisman et al., 1995), then aged rat should be impaired in learning this problem. The performance on simple, two-choice discriminations with no overlapping components should be spared, given that such learning can proceed normally in the absence of the hippocampus. 


\subsection{Materials and Methods}

Animals. Subjects were 29 female Brown Norway x Fisher 344 (BNxF344) hybrid rats (NIA/Harlan). At the start of the experiment, 10 rats were 3 months old, 10 were 12 months old, and 9 were 24 months old, weighing between 250$425 \mathrm{~g}$. One old rat died during the MR scanning procedures (which were performed prior to behavioral testing) due to pre-existing stroke. These rats are the same ones participating in Experiment 3, in which we assessed the spatial hippocampus-dependent performance of rats. The rats were group-housed in hanging plastic cages in a room with an ambient temperature of $21^{\circ} \mathrm{C}, 35 \%$ relative humidity, $12 / 12$ light/dark cycle, and had access to food and water ad libitum. All animals were cared for and tested at The University of Lethbridge vivarium according the National Institutes of Health guidelines and under the approval of the institutional animal care committees at the University of Lethbridge, University of Calgary, and the National Research Council which only approves studies that are in accordance with the Canadian Council on Animal Care guidelines.

Visual Water Task. The Visual Water Task (VWT) was used to train rats on the discrimination problems. Prusky et al. (2000) previously described this task in detail. Briefly, a trapezoidal metal tank $(140 \mathrm{~cm} \mathrm{~L} \times 80 \mathrm{~cm} \mathrm{~W} \times 25 \mathrm{~cm} \mathrm{~W}$ and 55 $\mathrm{cm} \mathrm{H}$ ) was filled with water to a depth of $15 \mathrm{~cm}$. The end wall of the tank was transparent. A midline divider $(40 \mathrm{~cm} \mathrm{H})$ was placed in the tank and extended 
$46 \mathrm{~cm}$ into the pool from the transparent wall. This divider separated two computer monitors (17" VGA; Viewsonic E7OF) that were positioned outside the transparent wall of the pool and used to present the stimuli. A transparent Plexiglas platform ( $37 \mathrm{~cm} \mathrm{~L} \times 13 \mathrm{~cm} \mathrm{~W} \times 14 \mathrm{~cm} \mathrm{H}$ ) was submerged at the end of one of the arms, and always paired with the correct $(+)$ stimulus. A computer program (Vista; http://www.cerebralmechanics.com) was used to control the right/left randomization pattern, record behavioral responses, and provide parameter control.

\section{Discrimination Training Procedures}

Qverview. Rats were shaped to associate swimming to the platform with escape from the water. The platform was located directly below the monitor displaying the rewarded (+) stimulus. At the beginning of each trial, animals were released into the pool facing the transparent wall, and allowed to swim to one of the stimuli. If choice was correct, rat was allowed to climb the platform and remain there for a few seconds before being returned to the holding cage. If incorrect, the rat was trapped in that arm of the pool for $10 \mathrm{~s}$ and then allowed to swim to the arm containing the platform before being removed from the pool. Trapping was not instituted on the test day. The choice was correct if animals swam to the platform without entering the arm displaying the non-rewarded stimulus. The rat was judged as entering the incorrect arm if the hind legs crossed into this arm of the pool, even if the rat had turned around before reaching the platform. The pattern of the rewarded stimulus/platform location 
was a left (L), right (R), LLRLRR sequence. The dependent variable was percentage of correct choices. The intertrial interval was approximately 3 minutes.

The overall design of the experiment is presented in Table 1. All rats were initially trained on a set of elemental discrimination problems (Figure 10, Set 1 ) following by the transverse patterning problem (Figure 10, Set 3 ). Each rat was trained and tested using the same procedures described above.

Elemental Discriminations. Rats were trained to solve three visual discriminations (Figure 10, Set 1: $A+B-, C+D-, E+F-$ ) using a stepwise approach. Training consisted of nine days in three phases ( 3 days for each phase, ), a test on day 10 , followed by a novel transfer testing on Day 11 . Phase 1 consisted of 3 days of training on Problem 1, 30 trials each. In Phase 2, Problem 2 was introduced and intermixed with Problem 1. Days 4 and 5 consisted of 5 trials of Problem 1, followed by 20 trials of Problem 2, and again by 5 trials of Problem 1. On Day 6 Problem 1 and 2 alternated every 5 trials for a total of 30 trials, starting with Problem 1. In Phase 3, Problem 3 was introduced and intermixed with Problems 1 and 2. On Day 7 rats received 5 trials of Problem 1, 20 trials of Problem 3, and 5 trials of Problem 2. Day 8 consisted of 5 trials of Problem 2, 20 trials of Problem 3, and 5 trials of Problem 1. Day 9 consisted of alternating blocks of 5 trials of each problem $(1,2$, and 3$)$. The test day, Day 10, consisted of randomly alternating problems on each trial 
$(3,2,1,2,1,3,2,3,1,3,1,2,1,2,3 \ldots)$, for a total of 30 trials, 10 of each problem.

Transverse Patterning. Transverse patterning (Figure 10, Set 3: X+Y-; Y+Z-; $Z+X-)$ training consisted of 7 days, followed by a test on Day 8,30 trials per day. Phase 1 consisted of 1 day of training on Problem $1(X+Y-)$. In Phase 2, Problem $2(Y+Z-)$ was introduced and intermixed with Problem 1 for 1 day, and consisted of 5 trials of Problem 1, followed by 20 trials of Problem 2, and again by 5 trials of Problem 1. On Day 3 Problem 1 and 2 alternated every 5 trials for a total of 30 trials, starting with Problem 1. In Phase 3, Problem $3(Z+X-)$ was introduced and intermixed with Problems 1 and 2. On Day 4 rats received 5 trials of Problem 1, 20 trials of Problem 3, and 5 trials of Problem 2. Day 5 consisted of 5 trials of Problem 2, 20 trials of Problem 3, and 5 trials of Problem 1. Day 6 consisted of alternating blocks of 5 trials of each problem $(1,2$, and 3$)$. The test day, Day 8 , consisted of randomly alternating problems on each trial $(3,2,1,2,1,3,2,3,1,3,1,2,1,2,3 \ldots)$, for a total of 30 trials, 10 of each problem.

\subsection{Results}

Elemental Discriminations. Figure 15 presents the results for each problem of the elemental discrimination paradigm on the test day by group. By the completion of training rats were performing at a near-perfect level on all three 
problems. Age had no effect on performance, all rats were able to learn a set of three elemental discrimination problems. We performed a repeated measures ANOVA with Problem (3) as a within-subjects variable, age Group (3 levels: Young, Middle-aged, Old) as a between-subjects variable, and performance ( $\%$ correct) on each problem as a dependent variable respectively. There was a significant effect of Problem $(F(2,50)=7.545, p=.001)$. The Group X Problem interaction was not significant $(F(4,50)=.514, p>.7)$, and there was no significant difference between the age groups $(F(2,25)=.844, p>.4)$. Post hoc tests revealed that the performance of each group did not significantly differ from one another regardless of the problem at hand $\left(p^{\prime} s>1\right)$.

Transverse Patterning. Figure 16 presents the results of the transverse patterning paradigm . A repeated measures ANOVA was performed with Problem (3) as a within-subjects variable, age Group (3 levels: Young, Middle-aged, Old) as a between-subjects variable, and performance ( $\%$ correct) on each problem as a dependent variable respectively. There was a significant Group X Problem interaction $(F(4,50)=6.285, p<.001)$. Both young and middle-aged rats were able to learn the transverse patterning problem, but not the old rats. The effect of Problem was significant as well $(F(2,50)=17.156, p<.001)$. The difference in performance between the age groups was also significant $(F(2,25)=$ $106.677, p<.001)$. Post hoc tests revealed that for each of the three transverse patterning problems, the performance of the Young and Middle-aged groups did not significantly differ $\left(p^{\prime} s>, 6\right)$. On the other hand, the Old group performed 
significantly worse from both the Young and the Middle-aged group on each of the three problems $(p<.001)$.

\subsection{Discussion}

The results of the present experiment support our prediction, that old age would significantly and selectively affect performance on the transverse patterning problem. When tested simultaneously on all three problems that comprise the transverse patterning, old rats had a significant deficit in performance while young and middle-aged rats had little trouble learning the problems. In contrast, all age groups performed similarly when tested concurrently on the three problems comprising elemental discriminations. Interestingly, this is the exact pattern of age-related performance deficits on the transverse patterning task that we have previously observed in humans (Driscoll et al., 2004a); no problems in learning the task for the young and the middle-aged participants whereas elderly participants were significantly impaired.

We tested the visual acuity of young and old FBNF1 rats in Experiment 1, the results of which suggest that the performance deficit on visual discriminations is not biased by poor vision in old age. This assumption is further supported by the fact that the same rats are able to discriminate between concurrent elemental discriminations, which can be solved in the absence of the hippocampus. In addition, successful performance by all rats on elemental discriminations 
suggests the absence of a potential bias due to potential motor or motivational problems that conceivably accompany aging.

These data, coupled with impaired retention of spatial information, point to a broad age-related deficit in hippocampus-dependent learning and memory that is not specific to the type of information or the test format employed. The same findings can also be used to support certain properties of hippocampal function in general. For example, Rudy and Sutherland (1995) posit that increasing the ambiguity between the stimuli should increase the reliance on configural/conjunctive associations to solve the problem, and in turn the hippocampus is the structure that allows these associations to disambiguate stimulus compounds. Our current results are in agreement with their position.

Hence, the study of hippocampus-dependent learning and memory in relation to aging will not only help define the age-related hippocampal mnemonic dysfunction, but should also enhance our understanding of the contributions that the hippocampus makes to learning and memory. In addition, we've established two different hippocampus-dependent tasks, one spatial and the other nonspatial in nature, that we can employ to test hippocampus-dependent learning and memory in rats and humans alike.

Now that we've established that hippocampus-dependent learning and memory deficits are present in aged FBNF1 rats, we proceed with assessment of hippocampal integrity on several different levels. First is the structural and 
neurochemical assessment of hippocampal integrity in vivo in the same rats that we characterized behaviorally in the current study and in Experiment 3. 


\section{Experiment 6 - Magnetic Resonance Imaging and Spectroscopy}

\section{Are the age-related alterations in hippocampal structural or functional integrity observable in vivo?}

\subsection{Background}

An in vivo magnetic resonance imaging (MRI) study employing a high field magnet was undertaken to help develop a rat model of aging and to evaluate both MRI-based volumetric measurements and metabolic profile in the rat in vivo. Volumetric estimations based on structural MR images are commonly used in human research in a wide variety of conditions. In animal research anatomical assessments are, on the other hand, customarily conducted post mortem. MR imaging, however, has an advantage over post mortem analyses. For example, MR allows not only the assessment of structural integrity in vivo, but allows us to study the changes longitudinally. Indeed, in vivo MRI has been employed to identify structural degradation in several experimental rodent models (Assaf et al., 1997; Baba et al., 1990; Bouilleret et al., 2000; Norman et al., 1991; Saunders et al., 1989; Wolf et al., 2002). In addition, some studies even reported an agreement between their MRI and histological observations (Allegrini and Sauer, 1992; Ben Horin et al., 1996; Minematsu et al., 1992). 
To the best of our knowledge, no volumetric studies of rat brains using MRI exist in relation to aging, regardless of the fact that such measurements are routinely performed in studies of human aging. The present study has two of goals in relation to MRI: first, to develop a reliable rat model of normal human aging, and to measure hippocampal volumes in these animals in vivo using MRI images.

Moreover, proton magnetic resonance spectroscopy $\left({ }^{1} \mathrm{H}\right.$ MRS) of the brain provides a noninvasive way of measuring metabolic information from amino acids, amines, sugars, and other compounds involved in high-energy metabolism. In recent years neurospectroscopy of the human brain has shed new light on metabolic mechanisms operating during normal brain development. $\mathrm{N}$-acetylaspartate (NAA) resonance contributes to the most prominent signal in ${ }^{1}$ H MRS.

NAA is almost exclusively present in the central nervous system and is predominantly located in pyramidal neurons, oligodendrocyte type 2 astrocyte progenitor cells, and in mature and immature oligodendrocytes. Given that NAA has been histologically localized primarily to neural tissue (Birken and Olendorf, 1989; Moffet et al., 1991), NAA levels are often thought to either reflect neuronal atrophy, neuronal density, or signify viable brain cells. Given that this substance is synthesized in the mitochondria from aspartate and acetyl-CoA (Benuch and D'Adamo, 1968), and metabolized by aspartoacylase into aspartate and acetate in the cytosol (D'Adamo et al., 1973), recently it has been 
suggested that NAA should be viewed as a marker of neuronal metabolic function.

In recent years, there are reports of decrease of NAA levels in the absence of neuronal loss (Jenkins et al., 2000). In addition, a reversible depletion of NAA levels either due to treatment (Kalra et al., 1998) or spontaneously (De Stefano et al., 1995), suggesting that even though a decrease in NAA is observed in these conditions at baseline - it could not simply reflect neuronal loss, given the turnaround. Instead, a depression in metabolic function has been offered as an alternate explanation. Moreover, Canavan's disease, an autosomal recessive leukodystrophy due to abnormal aspartoacylas clinically characterized by hypotonia, head lag, and macrocephaly without hydrocephalus and biochemically characterized by aspartoacylase deficiency, is marked by a large elevation in NAA levels, an elevation that clearly does not reflect an increase in neuronal density. Together, these findings indicate that both high and low levels of NAA can denote neuronal dysfunction.

The shift has been made from interpreting NAA as a putative neuronal marker to a marker for normally functioning neurons. Alternatively, because it has been demonstrated that mitochondrial NAA synthesis is affected by mitochondrial toxins (Bates et al., 1996), NAA depletion could more simply reflect a deficit in the mitochondrial oxidative phosphorylation processes. Dautry and colleagues (2000) demonstrated that NAA depletion, at least in the early stages, occurs in 
the absence of cell death suggesting it to be a marker of neuronal dysfunction. Needless to say, the function of NAA remains a subject of considerable debate.

Decreases in hippocampal NAA have been reported repeatedly in aged humans (e.g. Driscoll et al., 2003a; Schuff et al., 1999), however, it has not been investigated thus far in aged rodents. In order to evaluate the neurochemical profile of namely NAA, Choline, and Creatine, we performed ${ }^{1} H$ MRS in both right and left hippocampus of young, middle-aged, and old FBNF1 rats. In addition, post mortem assessment of the same tissue will be described in Experiment 7, and may shed additional light on the function of NAA in general.

\subsection{Materials and Methods}

Animals. The same animals that participated in Experiments 3 and 5 (i.e. were assessed on a spatial and non-spatial hippocmapus-dependent learning and memory tasks) are involved in the imaging studies as well. Rats were divided into 3 groups: Young ( $N=10$; age 3 months at the start of the experiment), Middle-aged ( $N=10$; age 14 months at the start of the experiment), and old ( $N=10$; age 24 months at the start of the experiment) group. All animals were housed in pairs in hanging plastic cages, in a room with an ambient temperature of $21^{\circ} \mathrm{C}$ and $35 \%$ relative humidity, $12 / 12 /$ light dark cycle. Food and water were available ad libitum. All procedures were approved by the institutional committees on animal care at the University of Lethbridge, University of 
Calgary, and the National Research Council of Canada which only approve procedures that are in accordance with the Canadian Council on Animal Care and were conducted in agreement with the National Institutes of Health guidelines.

Procedures related to MR imaging and spectroscopy were conducted at the Experimental Imaging Centre, University of Calgary. Rats were temporarily transported to the University of Calgary vivarium where they were maintained and allowed to acclimate for one week. Scanning for each group of rats was also completed over a period of a week. One old rat died during the MR scanning procedures (which were performed prior to behavioral testing) due to an interaction between anesthesia and what turned out to be a large brain damage probably caused by a massive stroke. Even though the scan was completed, this animal was removed from analysis due to massive brain damage.

Animal Preparation Animals were first anesthetized with $4 \%$ isofluorane while breathing spontaneously. For maintenance of anesthesia during the experiment, isofluorane was reduced to $1.5 \%$ with slight correction for body weight. Respiration and heart rate were monitored throughout the experiment. The body temperature was also monitored throughout the scan via rectal probe, and maintained at $37 \pm 0.5^{\circ} \mathrm{C}$ using heating pad. Each rat was placed in a plastic cradle and the head was immobilized.

MRI Procedure. Imaging was performed using a $9.4 \mathrm{~T} / 21 \mathrm{~cm}$ horizontal bore magnet with an Avance console (Bruker, Germany). A sagittal scout image was 
acquired to ensure proper image alignment. A spin echo sequence (TR $=2 \mathrm{~S}$, TE $=40 \mathrm{~ms}, 4$ averages, $1 \mathrm{~mm}$ slice thickness, no gap, $256 \times 256$ resolution matrix, FOV $=2.5 \mathrm{~cm}$ ) was employed for acquisition of T2-maps in a coronal plane. A representative MRI image containing the dorsal hippocampus is presented in Figure 17. A total of 20 coronal sections were acquired and used for volumetric analyses. Anatomical images were acquired with a quadrature volume coil of $35 \mathrm{~mm}$ ID and $40 \mathrm{~mm}$ length (custom built by the Institute for Biodiagnostics/ National Reseach Council of Canada; IBD/NRC). The image acquisition took $17 \mathrm{~min} 4 \mathrm{sec}$.

Volumetric Analysis. The MRI scans were transferred to a desktop computer. Volumetric analysis was performed using in-house image analysis software (Marevisi, http://www.ibd.nrc.ca/english/bdo_e_marevisi.htm) developed by IBD/NRC. This software is a PC-based program with a graphic user interface, which allows the user to manually outline regions of interest (ROI) and obtain calculations of an area for the specific ROI. Coronal images were used for the volumetric analysis. For all ROIs, the measures for both hemispheres were combined.

The hippocampus (cornu ammonis and dentate gyrus) was manually outlined on coronal sections from rostral to caudal. The starting rostral slice was approximately $2.30 \mathrm{~mm}$ posterior to bregma and was defined by the CA and DG, and coincided with the dorsal hippocampal commissure. The caudal boundary was defined by the loss of contrast between the external capsule and the 
subiculum, the absence of DG, and the clear separation between the two cerebral hemispheres. This level corresponds to approximately $-6.72 \mathrm{~mm}$ from bregma. The hippocampal formation was measured on 6 consecutive slices in all animals. We also obtained a measure of whole brain volume by tracing all the images. Whole brain volume (or intracranial volume, ICV) was also used to calculate normalized hippocampal volumes. Three measures resulted from volumetric quantitation (all in $\mathrm{mm}^{3}$ ): intracranial volume (ICV), absolute hippocampal volume, and normalized hippocampal volume (to ICV).

1H MRS Procedure. In vivo single voxel localized ${ }^{1} \mathrm{H}$ MRS was performed using the same 9.4 T Bruker system employed for MR imaging. A STEAM sequence (parameters: $\mathrm{TE}=20 \mathrm{~ms}, \mathrm{TR}=2 \mathrm{~s}, \mathrm{TM}=26 \mathrm{~ms}, \mathrm{NEX}=1024, \mathrm{SW}=10000 \mathrm{~Hz}$ ) was used. We tailored the voxel size (width $2 \mathrm{~mm}$, height $2 \mathrm{~mm}$, depth $3 \mathrm{~mm}$ ) using coronal T2-maps, to minimize CSF partial volume effects as well as the contribution of the tissue outside of the hippocampus. The spectra were acquired independently from left and right voxels centered on the hippocampi respectively, and the acquisition time was $34 \mathrm{~min} 8 \mathrm{sec}$ per voxel. Voxel position is presented in Figure 18A. Spectroscopy was conducted with the help of a single turn ellipsoidal surface coil with $20 \times 23 \mathrm{~mm}$ dimensions (custom built by IBD/NRC).

Metabolite Quantification. A time-domain fitting strategy, AMARES (Advanced Method for Accurate, Robust, and Efficient Spectral Fitting), of short echo time in vivo ${ }^{1} \mathrm{H}$ MR spectra of normal rat brain was used to successfully quantify the 
information. Time domain analyses provided consistent results with wellresolved peaks. NAA, Cho, and Cre were quantified by jMRUI analysis (Naressi et al., 2001; http://www.mrui.uab.es/mrui), which incorporated the algorithms and graphical interface, and were expressed in institutional values. Representative spectra are presented in Figure 18B.

\subsection{Results}

MRI-Volumetrics. The Group differences in $\operatorname{ICV}(F(2,27)=14.327, p<.001)$ were significant, such that the ICV was the highest in the old animals (see Figure 19). Post hoc tests revealed that the ICV significantly differed between the Young and Middle-aged $(F(1,19)=20.226, p<.001)$, and the Young and Old $(F(1,17)=17.329, p=.001)$ rats, but not between the Middle-aged and Old group $(p>4)$. Hippocampal volume measurements were averaged for the left and right hippocampus, as there were no hemispheric differences. There was a significant difference between the age groups in both absolute $(F(2,27)=$ $4.018, p=.031 ;$ Figure 20$)$ and normalized $(F(2,27)=7.274, p=.003$; Figure 21) hippocampal volumes, such that the aged group had smallest hippocampi. Absolute hippocampal volume differed between the Young and Middle-aged rats $(F(1,19)=5.61, p=.029)$, and the Young and Old $(F(1,17)=7.042, p=$ .017) rats, but not between the Middle-aged and Old group $(p>.4)$. Similarly, normalized hippocampal volume differed between the Young and Middle-aged groups $(F(1,19)=11.545, p=.003)$, and the Young and Old $(F(1,17)$ 
$=11.497, p=.004$ ) rats, but not between the Middle-aged and Old group ( $p>$ 4).

Metabolite Quantification. Metabolites, namely NAA, Cho, and $\mathrm{Cre}$, have been quantified separately for the left and right voxel centered on the hippocampus. In the left voxel, we found no age-related differences in NAA $(p>.1 ; M=9.45)$, Cho $(p>.8 ; M=1.67)$, or Cre $(p>.1 ; M=7.16)$. Similarly, there were no agerelated differences in NAA $(p>.5 ; M=9.41)$, Cho $(p>.6 ; M=1.85)$, or Cre $(p$ $>.8 ; M=7.25)$ in the right voxel either.

\subsection{Discussion}

We imaged rat brain with a high field-strength magnet. This provided us with scans of sufficient anatomical contrast to determine the volume of the hippocampus, as well as the total intracranial volume. Our results suggest that both absolute and normalized hippocampal volume, obtained by quantifying structural MR images, was significantly lower in both the middle-aged and old rats in vivo. These findings suggest alterations in structural integrity of the hippocampus that begin early on in adulthood and continue into old age. These results are in line with our previous findings in humans (Driscoll et al., 2003a), that hippocampal volumes observed by MRI are significantly lower in elderly people compared to the young ones. 
Our results suggest that neurochemical profile in the hippocampus, namely the levels of NAA, Cho, and Cre remain unaltered with age in the hippocampus of FBNF1 rats. More specifically, the fact that NAA remains stable suggests that neuronal metabolic function in the hippocampus is not compromised with age, at least not in this strain of rat. Although ${ }^{1} H$ MRS is routinely used to study aging in humans, to the best of our knowledge, the present study is the first application of this technique to study hippocampal aging in the rat. Our findings of stable levels of metabolites under investigation could simply reflect the cross-species differences. Another possibility is that there is variability in chemical profiles between the strains of rat. Both issues remain to be resolved in the future. In addition, one must consider the possibility that the tissue other than the hippocampus may be contributing to the MR spectrum (given the lower hippocampal volumes with age) and hence biasing the metabolite quantitation.

It is important to note that the fact that NAA remained stable in the aged rat hippocampus, despite the compromised structural integrity and behavioral deficits, is in direct contradiction to the human literature. This finding yet again brings into question the interpretation of NAA as a marker of neuronal viability and the interpretations of numerous studies linked to a decrease in NAA in the human brain related to various conditions and disease states. For example, in the human hippocampus, lower levels of NAA are routinely observed with age (e.g. Schuff et al., 1999), including our in own work (Driscoll et al., 2003a). In addition, studies in different patient cohorts (Brooks et al., 1997, 1999; Jung et al., 1998) and normal populations (Jung et al., 1999) using quantitative 
spectroscopy and an extensive neurocognitive battery suggest a significant relationship between neurochemical markers of brain integrity and brain function.

Overall, our results suggest that age-related structural deficits in the integrity of the hippocampal formation are apparent in vivo, as reflected by lower hippocampal volumes with age. Given that there were no differences in NAA, the overall functional integrity of neurons that remain in the old hippocampus seems to be preserved. Together, our findings lead us to believe that while the neurons that reside in the aged hippocampus retain their ability for normal metabolic functioning, the loss in the infrastructure is such that it is unable to provide the needed support for normal functioning, and we see such changes reflected in age-related deficits in hippocampus-dependent learning and memory. Allthewhile, magnetic resonance imaging has proven useful in tracking agerelated changes in the rat in vivo and is in line with human findings.

A couple of questions still remain: (1) what exactly does a decrease in structural volume signify, and (2) what does the change in NAA levels denote. In an attempt to answer the above-mentioned controversies, we next turn to electron microscopy procedures in order to assess the same hippocampal tissue post mortem. We will not only be able to assess the number of neurons, synapses, and mitochondria in the volume of interest, but in the end, we will be able to relate the investigation on a more cellular and ultrastructural level to our findings that were obtained using magnetic resonance technology. 
12. Experiment 7 - Light and Electron Microscopy

\section{Are the age-related alterations in hippocampal structural integrity observable post mortem?}

\subsection{Background}

According to the review by Geinisman et al. (1995), the most frequently observed structural alterations during normal aging involve the following: (1) loss of synapses; (2) neuronal atrophy; (3) astroglial hypertrophy, and (4) accumulation of lipofuscin pigment in neurons. For a long time, degeneration of neuronal and synaptic populations has been deemed a probable cause of agerelated learning and memory problems. To date, it is not known whether and what kind of an effect such changes have on memory and neuronal function in general.

Morphological changes in the number of neurons and synapses have been extensively investigated in search of structural substrates of memory dysfunction in normal aging (see Geinisman et al., 1995 for a review). Many of these studies, but not all, have reported an age-related loss of neurons and synapses in the hippocampus. More importantly, it has been demonstrated that the extent of the age-related morphological changes correlates with the severity 
of memory impairment. Morphological studies on neuron and synapse quantification in the hippocampus, in both humans and non-human animals, are plagued by a remarkable inconsistency in the results. Many of these reports have not used unbiased stereological techniques.

The advent of modern unbiased stereological techniques sidestepped the pitfalls of traditional stereological methodology and allowed for an unambiguous identification of section profiles of the objects being counted. The current state of the literature suggests that, using unbiased stereology and impeccable methodology, it has been convincingly demonstrated that neuronal loss does occur with chronological age in the human hippocampus (West, 1993). These results are however preliminary, and it remains to be characterized whether it is so for only certain regions of the hippocampal formation or if this process is more of a generalized one. In non-human animals, it remains to be resolved with unbiased stereology, whether there is a loss of hippocampal neurons with age. The loss of synapses in the non-human animals, however, has been confirmed using an unbiased stereological methodology, suggesting it as a candidate marker of age-related memory dysfunction.

Metabolic correlates of hippocampal structural changes related to aging are not well investigated and remain largely unknown. Metabolic function is receiving increased attention in aging research and can be easily assessed in vivo using magnetic resonance spectroscopy, but this imaging modality is most commonly used in human research. Mitochondria are important determinants of 
metabolism and can be easily quantified on an ultrastructural level using electron microscopy on post mortem tissue and readily applied to non-human animal research.

In Experiment 6, MRI and MRS allowed us to obtain an estimate of structural and functional neuronal integrity in the hippocampus in vivo. The use of such techniques in non-human animal research, however, is still somewhat unorthodox. In order to verify that structural changes are indeed present in the hippocampus in relation to old age we turn to a more traditional methodology for assessing structural integrity in non-human animals - a microscopical assessment of tissue post mortem. A light microscopy and the physical disector method (Sterio, 1984) were employed to get an estimate of the number of neurons in the hippocampus. In addition, we conducted an electron microscopy study in which serial sections were combined with the physical disector method (Sterio, 1984) to quantify synapses and mitochondria in the young ( 3 months old), middle-aged (14 months old), and old (26 months old) animals.

\subsection{Materials and Methods}

Animals. The same animals that participated in Experiments 3, 5, and 6 are involved in the light and electron microscopy studies as well. Rats were divided into 3 groups: Young ( $N=10$; age 3 months at the start of the experiment), Middle-aged ( $N=10$; age 14 months at the start of the experiment), and old 
( $N=8$; age 24 months at the start of the experiment) group. One old rat died during the MR scanning procedures (which were performed prior to behavioral testing) due to pre-existing stroke, and another one died of old age. Hence, all post mortem tissue analysis was done on eight animals in the old group. Animals were maintained and tested at the University of Lethbridge vivarium. All animals were housed in pairs in hanging plastic cages, in a room with an ambient temperature of $21^{\circ} \mathrm{C}$ and $35 \%$ relative humidity, $12 / 12 /$ light dark cycle. Food and water were available ad libitum. All studies were conducted according to guidelines provided by the National Institutes of Health and approved by the University of Lethbridge, University of Calgary, and the National Research Council of Canada, which only approve procedures that are in accordance with the Canadian Council on Animal Care. As previously mentioned, one rat suffered a stroke and died during MR scanning procedure. The brain of this rat was not processed for microscopy procedures.

Histological Methods. After completing behavioral testing, and magnetic resonance imaging and spectroscopy procedures, rats were injected with BrdU $(150 \mathrm{mg} / \mathrm{kg})$ and allowed to survive for 5 weeks. Following this 5 week period, all rats were sacrificed rats by overdose of sodium pentobarbitol pentobarbital (100 mg/kg i.p.) and perfused transcardially with phosphate buffered saline (PBS; $0.9 \%$ saline in $0.1 \mathrm{M}$ phosphate buffer) followed by $4 \%$ paraformaldehyde. Brains were extracted, and left and right hemispheres were separated. One hemisphere underwent immunohistochemical procedures while the other hemisphere underwent tissue preparation and embedding for electron 
microscopy. The hemisphere undergoing immunohistochemical procedures was stored in the perfusate solution overnight and then transferred to phosphate buffered saline solution. Hemisphere undergoing electron microscopy procedures was immersed in a fixative solution $(0.1 \mathrm{M}$ cacodylate buffer, $4 \%$ paraformaldehyde, $2.5 \%$ glutaraldehyde, and $2.5 \%$ dimethyl sulfoxide (DMSO) heated to $37^{\circ} \mathrm{C}$ for 5 minutes. The tissue was then transferred to the same solution at a temperature of $4^{\circ} \mathrm{C}$ and remained in it until dissected. The assignment of left or right hemisphere to either immunohistochemical or electron microscopy procedures was counterbalanced across all animals.

Tissue Preparation. The hemisphere was coronally sectioned on a vibratome into $300 \mu \mathrm{m}$ thick sections. Samples of tissue containing the dentate gyrus of the hippocampus were taken from each section. The tissue was then washed in $0.1 \mathrm{M}$ cacodylate, postfixed in $2 \%$ osmium tetroxide $/ 1.5 \%$ potassium ferrocyanide in $0.1 \mathrm{M}$ cacodylate buffer for 2 hours and stained en bloc with $2 \%$ uranyl acetate for 45 minutes. Samples were then dehydrated through a series of alcohols before being transferred into propylene oxide and gradually embedded in Eponate resin using standard resin embedding procedures.

One block of tissue, initially containing the granule cell layer of the dentate gyrus, was chosen at random from each animal and 32 coronal $1 \mu \mathrm{m}$ 'thick' sections were taken using a diamond knife and a microtome. The sections were stained with toluidine blue and used for light microscopy (Figure 22A) to estimates neuronal density in the molecular layer adjacent to the dorsal horn of 
dentate gyrus (Figure 22B). Following the collection of 'thick' sections, a small pyramid was trimmed such that each ultra-thin section spanned the inner molecular layer as well as the granule cell layer of the dorsal blade of the dentate gyrus, using toluidine stained thick sections from that block as a guide. The inner molecular layer was identified as an innermost third, the closest to the granule cell layer. From the pyramid, serial silver/gray ultrathin sections (70 $\mathrm{nm}$ ) were taken for electron microscopy using a diamond knife and an ultramicrotome. Sections were collected onto Formvar-coated copper grids and stained with uranyl acetate followed by lead citrate. Electron-microscopic measurements were taken from this tissue to obtain estimates of synaptic (Figure 23) and mitochondrial (Figure 24) density.

\section{Stereological Methods}

Estimating Changes. In conditions of stable neuron numbers changes in neuropil volume can be detected by estimating neuronal density. Measures of synaptic density alone may not reveal changes in synapse number. To measure changes in synapse number, the number of synapses per neuron can be calculated given that the density of neurons and the density of synapses respectively per unit volume have been obtained. Similarly, mitochondrial density, the number of mitochondria per neuron, and mitochondria per synapse were calculated. Unbiased stereological methods were employed in the present 
study to determine whether such structural changes within the molecular layer of the hippocampus exist in relation to aging.

Neuronal Density Measures. Using a computer assisted microscope and a stereology software package (Stereo Investigator), the physical dissector method (Sterio, 1984) was used to obtain estimates of neuronal density. Briefly, pairs of sections within each slide were compared in succession. The first section was considered the 'reference' section and its pair the 'look-up' section, and so on. In this method, neuronal nuclei that are present in one section are counted if they are not present in an adjacent section. Neuronal nuclei were identified by the presence of a central nucleolus within a pale nucleus, and by the oval to pyramidal shape of the surrounding cell soma, and counted using every other 1 $\mu \mathrm{m}$ section. Neurons were identified by multiple criteria including the presence of a central nucleolus, a pale nucleus surrounded by perikaryal cytoplasm and, frequently, by the oval to pyramidal morphology of the soma. Irregularly shaped small nuclei with extensive chromatin characteristics of glia were not counted. Within an unbiased counting frame $\left(37828 \mu \mathrm{m}^{2}\right.$ at $\left.40 \mathrm{X}\right)$, the number of neuronal nuclei present in the 'reference' but not the 'look-up' section (Q-) was counted. The disector volume of tissue $\left(75656 \mathrm{\mu m}^{3}\right)$ through which the cells were counted $\left(V_{\text {dis }}\right)$ was defined as $V_{\text {dis }}=\left(A_{\text {frame }}\right)(H)$, where $A_{\text {frame }}$ is the area of the counting frame and $H$ represents the thickness of the section $(1 \mu \mathrm{m})$ multiplied by the number of sections. Neuronal density was then determined by $\mathrm{N}_{\vee n e u r o n}=$ Q-/ $V_{\text {dis. }}$ Neuron density was subsequently used to determine the volume of neuropil associated with each neuron ( $\left.1 / N_{\text {vneiron }}\right)$. 
To calculate average neuron size, photographs of the region were acquired under a light microscope (40X) from the comparable section for each animal. Nine neurons were selected from each photograph and measurements of a diameter and an area were obtained for each neuron using NIH Image (http://rsb.info.nih.gov/nih-image). An average neuronal diameter and area were calculated for each rat in order to get a measure of neuronal size.

Synaptic Density Measures. The area under investigation was inspected with the electron microscope and an area of neuropil that was as much as possible free of nuclei was selected. A photomicrograph montage of four pictures, such that each micrograph represented one quadrant of a square, was taken from each position (at $6000 \mathrm{X}$ magnification). A section was skipped and the montage of the same area was taken in the subsequent section. The resulting negatives were printed at a final magnification of $\sim 23000 x$. Synaptic density ( $N_{v s y n a p s e}$ ) estimate was obtained from these micrographs by comparing the pairs of electron micrographs approximately $70 \mathrm{~nm}$ apart within the series. Synaptic density was measured using a physical dissector method on two sets of eight serially positioned electron micrographs per rat. Synapses that were present in the 'reference' section within an unbiased counting frame $\left(110.5 \mu \mathrm{m}^{2}\right)$ but not in the 'look-up' section, were counted. Synapses were identified by the presence of a synaptic membrane specialization and at least three presynaptic vesicles present in the presynaptic bouton and a presence of a post-synaptic density. Synaptic density was calculated as the number of synapses counted divided by 
the sample volume. From these measurement the number of synapses per neuron could be determined ( $\left[N_{\text {vsynapse }}\right]\left[1 / N_{\text {vneuron }}\right]$, Kleim et al., 1996), given that neuron count was previously made on the same tissue.

Mitochondrial Density Measures. The same methodology described above for measuring synaptic density was employed to obtain an estimate of mitochondrial density in the dentate gyrus. Neuron and synapse density were previously obtained on the same tissue allowing the calculation of the number of mitochondria per neuron and the number of mitochondria per synapse as well.

\subsection{Results}

Neuronal Density. Measures for neuronal density, neuronal diameter, and neuronal area were subjected to a one-way ANOVA respectively with Group as an independent variable. On average, a total of 19 comparisons were made to count 102 neurons, a total of $\sim 75 \mathrm{~mm}^{2}$. There was a significant effect of Group on neuronal density $(F(2,14)=5.603, p=.019$; Figure 25A). Follow up tests revealed that the young and the middle-aged group did not differ in respect to neuronal density $(p>.8)$. The difference between the young and old rats ( $F$ $(1,9)=27.899, p=.001)$, and the middle-aged and old rats $(F(1,9)=6.63, p$ $=.033$ ) respectively, was significant, such that neuronal density was compromised only in the Old group. 
Similarly, there was a significant effect of Group on the size of neuropil per neuron $(F(2,14)=4.728, p=.031$; Figure 25B). Follow-up tests revealed that the young and the middle-aged group did not differ in respect to neuronal density $(p>.85)$. The difference in neuropil per neuron between the young and old rats $(F(1,9)=22.545, p=.001)$ was significant, and a non-significant trend was present between the middle-aged and old rats $(F(1,9)=4.544, p=.066)$, such that the amount of neuropil per neuron was compromised only in the old group.

In addition, we obtained measures of neuronal area and diameter, and subjected them to a one-way ANOVA respectively with age Group as an independent variable. We found no group differences in either measure of neuronal size $(p>.5)$.

Synaptic Density. Approximately 32 dissector pairs were used to count an average of 135 synapses through a volume of $\sim 247 \mu \mathrm{m}^{3}$. Measures of synaptic density and the number of synapses per neuron were subjected to a one-way ANOVA respectively with Group as an independent variable. We found no Group differences in synaptic density $(p>.3)$ or in the number of synapses per neuron $(p>.25)$.

Mitochondrial Density. Approximately 32 dissector pairs were used to count an average of 140 mitochondria through a volume of $\sim 247 \mu \mathrm{m}^{3}$. Measures of mitochondrial density and the number of mitochondria per neuron were 
subjected to a one-way ANOVA respectively, with Group as an independent variable. We found no Group differences in mitochondrial density $(p>.8)$, in the number of mitochondria per neuron $(p>.28)$, or the number of mitochondria per synapse $(p>.2)$.

\subsection{Discussion}

The electron microscopy literature involving age-related brain changes is marked by contradictions, which are often attributed to the biased methodology employed, to the individual differences in aging, to a post-mortem delay, and to species and strain differences. We employed unbiased stereological methodology and the tissue was processed and post-fixed immediately after the animals were sacrificed. Measurements of neuronal, synaptic, and mitochondrial density were obtained from the same resin-embedded samples, therefore the effects of tissue shrinkage contributed equally to all density estimates and were unlikely to bias the data. Given that it has been widely accepted that there is neuronal atrophy in the aged human hippocampus, establishing whether aged rats exhibit cell loss of hippocampal neurons with unbiased stereological techniques is essential for the development of reliable animal models of human aging.

Employing unbiased stereological techniques, our results show a lower numeric neuronal density per sample volume and a lower amount of neuropil per neuron in the molecular layer adjacent to the dentate gyrus of the hippocampus in aged 
FBNF1 rat compared to both middle-aged and young rats. Neuronal count and the amount of neuropil per neuron seem preserved in middle-aged compared to young rats. At the same time, the neurons did not differ in their length or area in relation to age. Moreover, our results are in line with the finding that neuronal count is lower in the aged human hippocampi (West, 1993).

On the ultrastructural level, we found no age-related alterations in the number of synapses or the number of synapses per neuron in the dentate gyrus of the hippocampus of FBNF1 rats. Age-related loss of hippocampal synapses has been confirmed using unbiased stereology in the dentate gyrus, but not other regions of the hippocampal formation, and in some instances correlated with spatial memory impairment (see Geinisman et al., 1995). Our findings are inconsistent with the view that aging is related to synaptic loss, at least in this specific region of interest.

Also, synaptic efficacy has often been considered a basic mechanism underlying learning and memory. We have already described altered synaptic efficacy at least as measured by long term potentiation in Experiment 2. The already observed alterations in synaptic plasticity do not seem to be accompanied by a diminishing number of synapses, at least not in this region of interest. Previous work has found an age-related decline in synaptic numerical density per unit volume to involve only non-perforate but not perforated, axospinous synapses (see Geinisman et al., 1995 for a review). We did not make such a distinction in our current work. We can only assert with confidence that there is no change 
with age in overall synaptic density in the molecular layer directly adjacent to the granule cell layer of the dorsal horn of the dentate gyrus of the hippocampus.

Both quantity and size of mitochondria are important determinants of metabolism (Mjaatvedt and Wong-Riley, 1991; Wong-Riley, 1989). Here we quantified mitochondria using the same electron micrographs produced for estimating synaptic density. We found no age-related alterations in mitochondrial density or the number of mitochondria per synapse in the dentate gyrus of the hippocampus of the FBNF1 rats. The number of mitochondria is thought to be directly related to metabolic capacity (Wong-Riley, 1989). Hence, our results suggest that metabolic activity is preserved in the hippocampus of aged FBNF1 rats. Furthermore, because the number of mitochondria per synapse was preserved as well, synaptic activity in this region should be preserved. 


\section{Experiment 8 - Neurogenesis}

Does age affect neurogenesis, and if yes, do such changes further affect hippocampal function?

\subsection{Background}

Historically, it was commonly accepted that neurogenesis occurs only early on in development. Recently, however, it has been confirmed that neurogenesis within the hippocampal formation continues throughout adulthood (Altman and Das, 1965; Kaplan and Hinds, 1977; Lie et al., 2004). In addition, it turns out that most of the newly born cells express neuronal markers, receive synaptic inputs, extend axons along the mossy fibers, and possess similar electrophysiological properties to those of mature neurons in the granule layer of the dentate gyrus (see Kempermann et al., 2004; Lie et al., 2004 for reviews).

The neurogenesis levels in the dentate gyrus of the hippocampus are reported to decline with age in the rat (Gould et al., 1999a; Kempermann et al., 1998; Kuhn et al., 1996). Neurogenesis has also been implicated as a potential contributor to normal learning and memory processes (Gould et al., 1999c). Efforts have been made to correlate age-related neurogenic and cognitive dysfunction related to the hippocampus, but these efforts did not prove to be as straightforward or 
as fruitful as might be hoped. Recent studies, while consistently reporting a reduction in neurogenesis and behavioral impairments in the context of aging, fail to detect a clear relationship between the two (Bizon et al., 2004; Merrill et al., 2003). Most have assessed, however, only the rate of proliferation. Thus, it has been suggested that possibly survival, rather than the proliferation, is more important when it comes to contributing to hippocampus-dependent functioning (Drapeau et al., 2003).

Hippocampal neurogenesis generates new neurons that become incorporated into the dentate gyrus throughout adulthood. The precursor cells in the dentate gyrus initiate adult hippocampal neurogenesis and it results in new granule cell neurons. Several developmental milestones take place between these two end points. The term 'neurogenesis' is used as a term that encompasses the entire multi-step process, and not just the division of neuronal cell progenitors. The present experiment was designed to assess three aspects of hippocampal neurogenesis: first, cell proliferation in the subgranular zone using Ki67 labeling, a marker for cycling cells; second, neuronal differentiation using doublecortin, a marker of immature and migrating neuroblasts; and third, survival of recently born hippocampal neurons using a long time interval between BrdU injection and labeling. Finally, the hypothesis that the deficit in hippocampus-dependent tasks is associated with changes in neurogenesis within the dentate gyrus in the context of aging will be evaluated. 


\subsection{Materials and Methods}

Animals. The same animals that participated in Experiments 3, 5, 6, and 7 also participated in the current study. Rats were divided into 3 groups: Young (age 3 months at the start of the experiment), Middle-aged (age 14 months at the start of the experiment), and Old (age 24 months at the start of the experiment) group. Animals were maintained and tested at the University of Lethbridge vivarium. All animals were housed in pairs in hanging plastic cages, in a room with an ambient temperature of $21^{\circ} \mathrm{C}$ and $35 \%$ relative humidity, $12 / 12 /$ light dark cycle. Food and water were available ad libitum. All procedures were approved by the University of Lethbridge, University of Calgary, and the National Research Council of Canada, which only approve procedures that are in accordance with the Canadian Council on Animal Care, and were carried out in accordance with the NIH guidelines. One animal in the Old group died following the MR procedures, and another one died of old age during the 5 week period between the BrdU administration and the rats being sacrificed. The immunohistochemistry reported involves seven rats in the Old group.

Injection Regiment. Four weeks after the completion of all testing procedures rats were injected intraperitoneally with Bromodeoxyuridine (BrdU; Sigma, 150 $\mathrm{mg} / \mathrm{kg}$ ) dissolved in saline for three consecutive days, twice per day, $10 \mathrm{hrs}$ apart. Rats were allowed to survive for five weeks following the final BrdU injection. 
Tissue Processing. For perfusion, all rats were sacrificed rats by overdose of sodium pentobarbitol pentobarbital (100 mg/kg i.p.) and perfused transcardially with phosphate buffered saline (PBS; $0.9 \%$ saline in $0.1 \mathrm{M}$ phosphate buffer) followed by $4 \%$ paraformaldehyde. Brains were extracted and left and right hemispheres were separated. Only one hemisphere was to undergo immunohistochemical procedures while the other hemisphere was to undergo tissue preparation and embedding for electron microscopy. The assignment of left or right hemisphere to either immunohistochemical or electron microscopy procedures was counterbalanced across all animals. The hemisphere undergoing immunohistochemical procedures was stored in perfusate solution overnight and then transferred to phosphate buffered saline. Brains were stereotaxically blocked in the coronal plane, and serial coronal sections were cut on a freezing microtome at $50 \mu \mathrm{m}$. One set of every fifth section was immuno-double-labeled for BrdU and neuronal marker NeuN. The second set of every fifth section was immuno-labeled for doublecortin (DCX), a marker for immature and migrating neuroblasts. The third set of every fifth section was immuno-labeled for Ki67, a marker for cycling cells.

\section{Immunohistochemistry}

BrdU/NeuN. The following DNA denaturation protocol was followed to visualize BrdU-labeled nuclei before immunolabeling: 2-hour incubation in $50 \%$ formamide/2X SSC buffer $\left(0.3 \mathrm{M} \mathrm{NaCl}, 0.03 \mathrm{M}\right.$ sodium citrate) at $65^{\circ} \mathrm{C}$, rinsed 
twice for $5 \mathrm{~min}$ in $2 \times \mathrm{SSC}, 30 \mathrm{~min}$ incubation in $2 \mathrm{M} \mathrm{HCl}$ at $37^{\circ} \mathrm{C}$, and 5 washes in phosphate buffered saline (PBS) over 2 hours. Immunohistochemistry for BrdU was performed using a rat anti-BrdU antibody (Accurate Chemical, Westbury, NY) at a concentration of $1: 250$.

Immunohistochemistry for NeuN was performed using a mouse anti-NeuN antibody (Chemicon, Temecula, CA) at a concentration of 1:500. For immunolableing, sections were placed in a solution of $2 \%$ normal goat serum (NGS) and $0.3 \%$ Triton $X-100$ in PBS (blocking solution).

The primary antibodies for both BrdU and NeuN were diluted in the blocking solution. Sections were then placed in the primary antibodies diluted in the blocking solution overnight ( $19 \mathrm{hrs}$ ). After three 5-minute rinses in PBS, the sections were incubated for 1 hour with secondary antibodies (goat anti-rat, 1:500; and goat anti-mouse, 1:250; Molecular Probes, Eugene, OR) diluted in 5X PBS. After three more 5-minute rinses with PBS, the sections were placed in streptavidin (Alexa 568; Molecular Probes, Eugene, OR) at a concentration of 1:500 for 45 minutes. Finally, sections were rinsed with PBS, mounted on gelatin-coated slides, air dried, and coverslipped. Specificity of both the rat antiBrdU and mouse anti-NeuN antibodies was verified by omitting the primary and secondary antibodies respectively, which resulted in a loss of cellular labeling.

BrdU-labeled cells that also expressed NeuN (Figure 26) throughout the dentate gyrus were detected with a laser-scanning confocal microscope lasers (Zeiss 
LSM Microsystem; Axiovert 100 TV). Sections were optically sliced in the Z plane at $1 \mu \mathrm{m}$ intervals. The data were transferred to a desktop computer and viewed with Northern Eclipse software (http://www.empix.com), which also allowed a reconstruction of images in 3D. All double-labeled cells were rotated in orthogonal planes to verify double-labeling. BrdU/NeuN labeling procedures were performed on the tissue obtained from 10 young, 10 middle-aged, and 7 old rats.

Doublecortin ( $\mathbf{D C X}$ ). Sections were incubated overnight ( $20 \mathrm{hrs}$ ) at room temperature with doublecortin (DCX, 1:250, Santa Cruz Biotechnology, Santa Cruz, CA) in $0.3 \%$ Triton $X-100,0.2 \%$ normal rabbit serum (NRS) in PBS. Sections were rinsed $3 \mathrm{X}$ in PBS, and placed in a secondary biotinylated anti-goat IgG (1:500) for $1 \mathrm{hr}$ at room temperature. Sections were again rinsed $3 X$ in PBS, and processed for immunofuorescence in streptavidin (Alexa 488, 1:500, Molecular Probes, Eugene, OR) for $45 \mathrm{~min}$. Sections were rinsed in PBS, mounted on gelatin-coated slides, air dried, and coverslipped. The sections were viewed on a Zeiss Axioskop Z microscope under fluorescence. Cell somata were identified and counted under a $40 \mathrm{X}$ objective (Figure 27). DCX-labeling procedures were performed on the tissue obtained from 10 young, 10 middleaged, and 7 old rats.

Ki67. Sections were incubated overnight ( $\sim 20 \mathrm{hrs}$ ) at room temperature with Ki67 (1:1000; Novacastra, UK) in $0.3 \%$ Triton $X-100$, and $0.2 \%$ normal donkey serum (NDS) in PBS. Sections were rinsed $3 X$ in PBS, and placed Cy3 
Conjugated Affinipure Donkey Anti-rabbit IgG (1:250; Jackson Laboratories; West Baltimore Pike, PA) for $45 \mathrm{~min}$ at room temperature. Sections were rinsed in PBS, mounted on gelatin-coated slides, air dried, and coverslipped. The sections were viewed on a Zeiss Axioskop $Z$ microscope under fluorescence. Ki67-labeled cells were identified and counted under a 40X objective (Figure 28). Ki67 procedures were performed on the tissue obtained from 9 young, 9 middle-aged, and 7 old rats.

\subsection{Results}

BrdU/NeuN, One-way ANOVA was performed with Group (Young, Middle-aged, and Old) as an independent variable and the number of BrdU-labeled cells or BrdU/NeuN double-labeled cells as a dependent variable respectively. We found a significant Group difference in the total number of BrdU-labeled cells $(F(2,25)$ $=8.393, p=.002$; Figure 29) five weeks after the last BrdU injection, such that the number of BrdU-labeled cells was the highest in the young rats, lower in the middle-aged, and the lowest in the old rats. Middle-aged rats had $20 \%$ of BrdUlabeled cells, and old rats had $3 \%$ of BrdU-labeled cells compared to the number observed in the young rats five weeks after the last injection. Follow up tests revealed that this difference was significant between young and middle-aged rats $(F(1,18)=7.891, p=.012)$, young and old rats $(F(1,16)=8.989, p=$ .009 ), and middle-aged and old rats $(F(1,15)=7.871, p=.014)$. 
Also, there was an age difference in the number of BrdU/NeuN double-labeled cells $(F(2,25)=9.522, p=.001$; Figure 30$)$ five weeks after the last BrdU injection, such that the number of BrdU/NeuN double-labeled cells was the highest in the young rats, lower in the middle-aged, and the lowest in the old rats. The number of double-labeled BrdU/NeuN cells in middle-aged rats was $17 \%$, and in old rats $6 \%$ that of the young ones five weeks after the last BrdU injection. Follow up tests revealed that the difference was significant between young and middle-aged rats $(F(1,18)=9.615, p=.006)$, young and old rats ( $F$ $(1,16)=9.789, p=.007)$, but not middle-aged and old rats $(p>.1)$.

Five weeks after BrdU injections, $47 \%$ of the BrdU-labeled cells in the young rats, $42 \%$ in the middle-aged rats, and $88 \%$ in the old rats were also doublelabeled with NeuN. It should be noted that in aged rats, even though we are seeing $88 \%$ of BrdU cells also double labeled with NeuN, this observation on average reflects a couple of double-labeled cells and it should not be interpreted as an increase in the rate of neuronal survival per se in these animals. Rather, it seems that the few cells that did survive after a period of five weeks in aged animals are primarily the ones that adopted a neuronal fate.

DCX. One-way ANOVA was performed with Group (Young, Middle-aged, and Old) as an independent variable and the number of DCX-labeled cells as a dependent variable. There was a significant Group difference in the number of DCX-labeled cells $(F(2,26)=402.315, p<.001$; Figure 31$)$, such that the young group had the highest number of DCX-labeled cells, followed by the 
middle aged group, and finally the old animals. Follow up tests revealed a significant difference between young and middle-aged rats $(F(1,16)=424.164$, $p<.001$ ), young and old rats $(F(1,16)=446.254, p<.001)$, and middle-aged and old rats $(F(1,16)=58.051, p<.001)$. The middle-aged rats had $20 \%$, and old rats had $5 \%$ of the number of DCX-labeled cells seen in the young group of rats.

Ki67. One-way ANOVA was performed with Group (Young, Middle-aged, and Old) as an independent variable and the number of Ki67-labeled cells as a dependent variable. There was a significant Group difference in the number of Ki67-labeled cells $(F(2,24)=24.188, p<.001$; Figure 32$)$, such that the young group had the highest number of Ki67-labeled cells, followed by the middle aged group, and finally the old animals. Follow up tests revealed a significant difference between young and middle-aged rats $(F(1,17)=26.073, p<.001)$, young and old rats $(F(1,15)=24.847, p<.001)$, but not the middle-aged and old rats $(F(1,15)=1.265, p<.28)$. The middle-aged rats had $20 \%$, and old rats had $14 \%$ of the number of Ki67-labeled cells seen in the young group of rats.

\subsection{Discussion}

Regulation of neurogenesis is considered an important indicator of neuroplasticity in the aging brain. In the current study, neurogenesis was 
assessed using three independent measures, one of proliferation - Ki67 (and possibly BrdU alone, but the animals survived for 5 weeks after BrdU administration), one of neuronal differentiation - DCX, and one of mature neuronal survival - BrdU/NeuN. Overall, our results suggest that aging is associated with a significant neurogenic reduction in the rat hippocampus, a finding that is consistent with other studies of aging and neurogenesis to date.

Our Ki67 results suggest a significant decrease in the rate of proliferation with age that begins sometime in adulthood, here specifically before 14 months of age in the FBNF1 rats. Our results are in agreement with numerous other studies suggesting a reduction in proliferation rate in relation to aging (e. g., Kempermann et al., 1998; 2002). Collectively, the reports of reduced proliferation rate with age would lead us to believe that the number of cells surviving in the aged hippocampus would be consequently reduced as well, given the lower pool of cells available to begin with. This seems to be the case, at least when it comes to neuronal differentiation and survival for a period of 5 weeks, given the dramatic reduction in both DCX-and BrdU/NeuN-labeled cells.

It seems plausible that the number of $D C X$ and BrdU/NeuN cells can be explained by reduced proliferation alone. Markers of proliferation, BrdU alone and $\mathrm{Ki67}$, provide us with similar estimates in the number of neurons born in the middle-aged and old rats respectively, compared to the young ones. For example, middle-aged rats had $20 \%$, and old rats had $3 \%$ of BrdU-labeled cells found in the young rats 5 weeks following BrdU administration. Similarly, 
middle-aged rats had $20 \%$, and old rats had $14 \%$ of Ki67-labeled cells. Markers of immature $(D C X)$ and mature neurons (BrdU/NeuN) provide us with similar estimates as well. Middle-aged rats had $20 \%$, and old rats had $5 \%$ of DCXlabeled cells found in the young rats. Middle-aged rats had $18 \%$, and old rats had $6 \%$ of BrdU/NeuN double-labeled cells found in the young rats. The only substantial discrepancy in these estimates appears in the measures of proliferation, BrdU vs. Ki67 (3\% vs. 14\% respectively).

One reason for this discrepancy could be that BrdU as implemented here is not a true measure of proliferation when assessed 5 weeks after BrdU administration. In order to properly assess the rate of proliferation with BrdU, animals need to be sacrificed approximately 2-3 hours following the BrdU administration. The goal here, however, was to use it for assessment of neuronal survival in concert with NeuN in the first place. Also, it is known that BrdU is associated with dilution as the time goes by and the cells that originally incorporated BrdU into their DNA continue to divide. In addition, lipofuscin - a cellular waste product which accumulates in the somata of aging animals, has recently been suggested as a possible bias in immunohistochemical methodology, and it is possible that it could affect one method of labeling but not the other.

Lipofuscin is a product of lipid peroxidation that accumulates over time in neuronal and non-neuronal cells. Lipofuscin accumulation in cells is thought to be a slow process that is taken as a sign of long-term oxidative stress. It has been suggested that the sustained induction of neurogenesis occurs in the 
cellular microenvironment that is characterized by reduced neuronal damage. More specifically, (Kempermann et al., 2002) found that lipofuscin deposits in neurons are significantly reduced by long-term exposure to environmental enrichment, and the amount of lipofuscin deposited has been linked to cognitive deficits. Lipofuscin was not measured here, but should be considered in the future studies of aging and neurogenesis as it has been recently suggested as a possible bias in immunohistochemical procedures in aged organisms.

In addition, in order to better describe the processes involving neurogenesis and elucidate a possible role for neurogenesis in the normally aging brain, we assessed the relationships that emerged between hippocampus-dependent behavior and other measures of hippocampal integrity with measures of hippocampal neurogenesis (Ki67-labeled cells, DCX-labeled cells, and BrdU/NeuN double-labeled cells). We describe these relationships in more detail in the Correlations section below. 


\section{Correlations}

In order to assess whether relationships between performance on hippocampusdependent tasks and measures of hippocampal structural and functional integrity obtained both in vivo and post mortem exist, we computed zero-order correlations (Table 2A).

Measures of performance on the Morris water task in the correlation calculations included latency and path length respectively on the last trial on the days that the platform was in a new location, latency and path length, respectively, on the first trial on the days the platform was in the same location as the previous day, a difference in the latency and path length respectively between the average of the last trial on the days the platform was in a new location and the first trial on the days platform was in the same location as the previous day (a measure of 24-hour retention), a difference in the latency and path length respectively between the average of the first trial and the last trial on the days the platform was in a new location (a more general measure of learning the new platform location across trials), and a difference in the latency and path length respectively between the average of the first trial and the third trial on the days the platform was in a new location (a more specific measure of new learning). For discrimination learning, performance measures were represented as a percent correct on each problem (3) for both elemental and transverse patterning discriminations. Additional measures that were included in correlation computations included absolute and normalize hippocampal volumes, neuronal 
density, synaptic density, mitochondrial density, hippocampal NAA, Cho, and Cre levels, the number of Ki67-labeled (proliferation), DCX-labeled (differentiation), BrdU-labeled, and BrdU/NeuN double-labeled cells (survival).

The performance on the two hippocampus-dependent tasks, one spatial and the other non-spatial in nature, correlated significantly. More specifically, success (percent correct) on the transverse patterning Problem 1 was significantly correlated with a difference score between trials 1 and 3 (learning) of the MWT on the day the platform was in a new location for both latency $(r=.454, p=$ $.015)$ and path length $(r=.454, p=.015)$. Success in learning Problem 1 was significantly correlated with performance on Problems $2(r=.634, p<.001)$ and $3(r=.691, p<.001)$ of the transverse patterning discriminations.

Transverse patterning Problem 2 performance significantly correlated with MWT place learning performance (latency) on the very first trial on days when the platform remained in the same location $(r=-.436, p=.020)$, with a difference score between trials 1 and 3 (learning) on the day the platform was in a new location for both latency $(r=.572, p=.001)$ and path length $(r=.440, p=$ .019), and with performance on Problems $1(r=.634, p<.001)$ and $3(r=$ $.860, p<.001$ ) of the transverse patterning discriminations.

Transverse patterning Problem 3 performance significantly correlated with place learning performance (latency) on the very last trial of the day the platform was in a new location $(r=-.430, p=.022)$, with place learning performance 
(latency) on the very first trial on days when the platform remained in the same location $(r=-.501, p=.007)$, with a difference score between trials 1 and 3 (learning) on the day the platform was in a new location for both latency ( $r=$ $.561, p=.002)$ and path length $(r=.404, p=.033)$, and with performance on Problems $1(r=.691, p<.001)$ and $2(r=.860, p<.001)$ of the transverse patterning discriminations.

Absolute hippocampal volume $\left(\mathrm{mm}^{3}\right)$ measured in vivo correlated significantly with a difference score between trials 1 and 3 (learning) on the day the platform was in a new location for both latency $(r=.508, p=.006)$ and path length $(r=$ $.508, p=.006)$. There was a significant relationship between absolute hippocampal volume and Problem 1 of elemental discriminations $(r=.418, p=$ $.027)$, and Problems $2(r=.432, p=.022)$ and $3(r=.446, p=.017)$ of the transverse patterning discriminations.

Absolute hippocampal volume also significantly correlated with the number of DCX-labeled cells $(r=.493, p=.009)$, with the total number of BrdU-labeled cells that remained after 5 weeks $(r=.408, p=.039)$, and with the total number of BrdU/NeuN double-labeled cells that survived for 5 weeks $(r=.447$, $p=.022$ ). In addition, absolute hippocampal volume significantly correlated with the whole brain volume $(r=-.592, p=.001)$, and normalized hippocampal volume $(r=.969, p<.001)$. 
Normalized hippocampal volume (to ICV) correlated significantly with latency to reach the platform on the first trial on days when the platform remained in the same location as the previous day $(r=-.378, p=.047$; Figure 33$)$, and with a difference score between trials 1 and 3 (place learning) on the day the platform was in a new location for both latency $(r=.474, p=.011)$ and path length $(r=$ $.474, p=.011)$. Normalized hippocampal volume also significantly correlated with Problem 1 of elemental discriminations $(r=.409, p=.031)$, and with performance on Problems $2(r=.471, p=.011)$ and $3(r=.488, p=.008$ Figure 34) of the transverse patterning discriminations.

Normalized hippocampal volume significantly correlated with the number of DCX-labeled cells $(r=.605, p=.001$; Figure 37$)$, with the total number of BrdU-labeled cells that remained after 5 weeks $(r=.390, p=.049)$, and with the total number of BrdU/NeuN double-labeled cells that survived for 5 weeks ( $r$ $=.425, p=.031$ ). In addition, normalized hippocampal volume significantly correlated with the whole brain volume $(r=-.771, p<.001)$, and absolute hippocampal volume $(r=.969, p<.001)$.

Whole brain volume (in $\mathrm{mm}^{3}$ ) correlated significantly with a difference score between trials 1 and 8 (place learning) on the day the platform was in a new location for latency $(r=.456, p=.015)$ and there was a trend for path length $(r$ $=.350, p=.068$ ) to reach the platform. Whole brain volume also significantly correlated with both normalized $(r=-.771, p<.001)$ and absolute hippocampal volume $(r=-.592, p=.001)$. 
The number of DCX-labeled cells significantly correlated with place learning performance (latency) on the last trial of the day the platform was in a new location $(r=-.543, p=.003$; Figure 35$)$. This relationship was significant in the senescent rats (middle-aged and old combined; $r=-.534, p=.027$ ), but not for young group (and it is in the opposite direction; $r=.506, p=.136$ ). The number of DCX-labeled cells also significantly correlated with a latency difference score between trials 1 and 8 (learning) on the day the platform was in a new location $(r=-.530, p=.004)$, with a difference score between trials 1 and 3 (learning) on the day the platform was in a new location for both latency $(r=.431, p=.025)$ and path length $(r=.431, p=.025)$ to reach the platform, and with performance on Problems $1(r=.429, p=.025), 2(r=.443, p=$ $.021)$, and $3(r=.481, p=.011$; Figure 36$)$ of the transverse patterning discriminations. The relationship between DCX and performance on Problem 3 of the transverse patterning task was significant in the elderly (middle-aged and old together; see Figure $36 ; r=.805, p<.001$ ), but not in the young rats ( $r=$ $.180, p=.619)$.

The number of DCX-labeled cells in the hippocampus also significantly correlated with absolute $(r=.493, p=.009)$, and normalized $(r=.605, p=.001$; Figure 37) hippocampal volumes, as well as with the total number of BrdU-labeled cells that remained after 5 weeks $(r=.612, p=.001)$, and with the total number of BrdU/NeuN double-labeled cells that survived for 5 weeks $(r=.642, p=.001$; Figure 38). 
There was a trend for the total number of BrdU-labeled cells that remained after 5 weeks $(p=.084)$, and with the total number of BrdU/NeuN double-labeled cells that survived for 5 weeks $(p=.091)$ to be significantly correlated with a difference score between trials 1 and 3 (learning) on the day the platform was in a new location on the MWT. The total number of BrdU-labeled cells present after 5 weeks was significantly correlated with absolute $(r=.408, p=.039)$ and normalized hippocampal volume $(r=.390, p=.049)$. The total number of BrdU/NeuN double-labeled cells present after 5 weeks also significantly correlated with absolute $(r=.447, p=.022)$ and normalized hippocampal volume $(r=.425, p=.031)$. The correlation between the total number of BrdUlabeled cells that were present after 5 weeks and the total number of $\mathrm{BrdU} / \mathrm{NeuN}$ double-labeled cells that survived for 5 weeks was also significant ( $r$ $=.958, p<.001)$.

The number of Ki67-labeled cells significantly correlated with performance on Problem 3 of the transverse patterning discriminations $(r=.456, p=.022$; Figure 39), and not with any measures of performance on MWT. The number of Ki67-labeled cells also significantly correlated with absolute $(r=.449, p=.024)$ and normalized ( $r=.599, p=.002$; Figure 40 ) hippocampal volumes. In addition, there was a significant relationship between the number of Ki67- and DCX-labeled cells $(r=.869, p<.001$; Figure 41$)$, but not with BrdU-labeled cells. 


\subsection{Partial Correlations}

We also performed another set of correlations with the same variables described above, but this time we controlled for age. A slightly different pattern of relationships emerged, which are described in more detail below.

Latency to reach the platform on the last trial on the days the platform was in a new location correlated with percent correct on the Problem 3 of transverse patterning discriminations $(r=-.638, p=.047)$. There was a relationship between performance on Problem 3 of transverse patterning discriminations with Problem $1(r=.744, p=.014)$, and a trend with Problem $2(r=.586, p=$ $.075)$

Hippocampal neuronal count was significantly correlated with performance on Problem $1(r=.752, p=.012)$ and Problem $3(r=.798, p=.007)$ of transverse patterning discriminations. Normalized hippocampal volume correlated with latency $(r=-.672, p=.033)$ to reach the platform on the last trial on the days the platform was in a new location, and a non-significant trend for path length $(r=.593, p=.071)$. There was also a relationship with absolute hippocampal volume $(r=.939, p<.000)$ and whole brain volume $(r=-.653, p$ $=.041)$. The total number of BrdU-labeled cells that remained after 5 weeks was significantly correlated with BrdU/NeuN double-labeled cells $(r=.919, p<$ $.001)$ 
There was a significant relationship between Choline levels in the right hippocampus and a difference score between trials 1 and 8 (learning) on the day the platform was in a new location for latency $(r=.639, p=.046)$, and a trend for path length $(r=.606, p=.063)$. Right hippocampal NAA levels significantly correlated with a difference score in path length to reach the platform between trials 1 and 8 on the days the platform moved to a new location $(r=.704, p=$ $.023)$.

Given that we observed relationships between neurogenesis and behavior, and hippocampal volume and behavior respectively, but no significant relationships between neuronal density and behavior (unless we control for age), we wanted to see what was driving these relationships. Conceivably, one could imagine the rate of neurogenesis mediating both neuronal density and the volume of the hippocampus. The reverse, of either the volume or the neuronal atrophy predicting the rate of neurogenesis, is hard to imagine.

Hence, another set of partial correlations was calculated including all the variables described above for zero-order correlations, but this time we controlled for the number of DCX-labeled cells. When the number of DCX-labeled cells was controlled for, the relationships between the measures of MWT $(p>.174)$ and visual discrimination performance $(p>166)$ respectively and measures of hippocampal volume were no longer present. Similarly, there was no relationship between neuronal density and measures of learning and memory on hippocampus dependent tasks $(p>.635)$. 
Similarly, we computed additional partial correlations that included all the variables previously described in the Correlation section and but this time we controlled for neuronal density. When the effects of neuronal density were controlled for, the relationships between hippocampal volume and measure of both spatial and non-spatial hippocampus-dependent learning and memory were no longer present $(p>.28)$. 


\section{General Discussion}

Collectively, our results suggest that aging is associated with deficits in both spatial and non-spatial hippocampus-dependent tasks. Behavioral deficits on hippocampus-dependent tasks are not altogether unexpected, given the observed age-related changes in hippocampal plasticity seen with age. In addition, aging of the hippocampus is accompanied by structural alterations on several different levels of description that can be observed both in vivo and post mortem. Furthermore, our results using a within-subjects experimental design show that the observed hippocampal structural changes predict the age-related dysfunction in learning and memory performance dependent on this brain region. Of equal importance is what was notobserved. The marked behavioral, physiological, and anatomical changes were not accompanied by a deficit in functional metabolic integrity as measured by magnetic resonance spectroscopy. In considering the current results, one should not forget that the laboratory rats live in environments that are impoverished compared to their natural habitat.

In considering the performance on hippocampus-dependent tasks, we observed deficits on both tasks employed here. Both middle-aged and old rats displayed a deficit in 24-hour retention of spatial information on the Morris water task. The age profile of performance is the same as the one we reported with humans in the virtual Morris water task (Driscoll et al., 2005). When it comes to learning and memory of non-spatial hippocampus-dependent information, a slightly different pattern of deficits emerges. Now, the performance of middle-aged rats 
is similar to that of a younger rat, and the deficit is only present in the old group. Again, we have previously reported this exact pattern of performance in relation to age on the same task in humans (Driscoll et al., 2004a). Together, our previous findings in humans and and current findings in the rat suggest that the spatial task may be more sensitive to alterations in hippocampal functional integrity related to aging, as the deficits can be observed early on in adulthood.

In respect to alterations in hippocampal anatomy, we found a reduction in the volume of the hippocampal formation in both middle-aged and old rats as observed by MRI. This is the first report of reduced hippocampal volume in the aging rat in vivo. Furthermore, our findings suggest that the alterations in hippocampal volume begin early in adulthood, sometime before 14 months of age in the FBNF1 rats, and seem progressive in nature. The results are also of importance in relation to validating our rat model of aging. Present MRI results are on agreement with numerous observations of volume reduction in the hippocampus of elderly humans (e.g., Driscoll et al., 2003a; Schuff et al., 1999). The volume in middle-aged humans in the context of aging, however, has not been systematically investigated and we are unable to draw a comparison in this age group at this point in time.

On a more general note, the success of our volumetric study also demonstrates the usefulness of MRI in rodent research, especially the possibility of investigating longitudinal age-related changes during the lifespan of the rat, a lifespan that is much shorter from that of a human and hence more conducive to 
investigation. The question, however, remains as to what may underlie the volume decrease. Two candidates become readily apparent: (1) cell death, and (2) the neurogenic reduction. Here we measured both. Historically, it has been presumed that the reduction in volume is a direct consequence of neuronal atrophy that is expected to accompany aging, but recently this view has been brought into question. We will discuss the possible sources of volume reduction in more detail shortly, but first we will consider the information obtained from the post mortem tissue.

In addition to structural age-related alterations observed in the hippocampus in vivo, we have also observed structural alterations in post mortem hippocampal tissue. We found a reduction in both neuronal density and the amount of neuropil per neuron in the hippocampal inner molecular layer of old rats, whereas no changes were observed in the young and middle-aged group. Whether the number of neurons in the human hippocampus is reduced in old age has been resolved to the satisfaction of many (West, 1993), and the resolution is that the neuronal density is indeed reduced in the elderly human hippocampus (see Geinisman et al., 1995). The jury is still out, however, on whether the same holds true for the aging rat hippocampus. Our current findings add to the body of literature that reports alterations in neuronal density in the hippocampus with old age and are in agreement with human findings. The studies reporting no changes in neuronal density in the aged rat hippocampus, however, should not be ignored. It remains to be resolved whether inconsistencies in this literature are dependent on the strain of rat used and the 
exact area of the hippocampus under investigation, both of which have been suggested as a possible source of discrepancies.

Nonetheless, it is easy to imagine how the loss in neuronal density may have a direct effect on hippocampal volume. The volume of the hippocampus in the aged rats was reduced by $11 \%$, while the neuronal density was reduced by $25 \%$. This reduction in neuronal density seems substantial enough to account for the decrease in volume observed with old age. This idea is further supported by the fact that the significant relationship between volume and performance on hippocampus-dependent tasks is no longer significant once we controlled for neuronal density. Yet the reduction in hippocampal volume was also present in middle-aged rats in the absence of altered neuronal density, suggesting that decreased neuronal density contributes but does not conclusively explain the reduction in volume of this structure with age.

Additional anatomical measures of the hippocampus were obtained from post mortem tissue in relation to neurogenic processes in order to assess proliferation, differentiation, and survival of recently born hippocampal neurons and its aftereffects, and to test the hypothesis that the deficit in hippocampusdependent tasks is associated with a reduction in neurogenesis within the dentate gyrus in the context of aging. Our results suggest that the rate of proliferation (Ki67), the number of newly born cells that have adopted an immature neuronal phenotype (DCX-positive), and the number of newly born cells co-expressing a mature neuronal marker NeuN five weeks after labeling are 
all reduced in the aged rat hippocampus, and such changes are already apparent in the middle-aged animals. Neurogenesis is rarely investigated in humans, mainly due to practical and methodological limitations. Nonetheless, a reduction in the proliferation rate in the aged human hippocampus has been noted (Eriksson et al., 1998).

As easy as it was to imagine how neuronal loss could account for a reduction in volume, it is almost as easy to imagine how the reduction in neurogenesis, in both proliferation and survival, could underlie both the reduction in neuronal density and the reduction in volume. A significant relationship between volume and performance on hippocampus-dependent tasks disappeared after controlling for $D C X$, suggesting the role for neurogenesis in maintaining the structural integrity of the hippocampus and performance on the tasks that depend on its integrity.

It remains to be investigated further whether it is the case that the cell loss increases with age and at some point neurogenesis can no longer keep up. It could be the case that the cell loss is steady throughout the adulthood, but the neurogenesis is reduced severely in old age and can no longer keep up with maintaining the structural integrity of the hippocampal formation. Of course, the third possibility remains, that both apoptosis and neurogenesis have been unfavorably affected by aging. Our results suggest that this may be the case, given the reduction in neurogenesis, and given the increase in cell loss as evident by decreased neuronal density. In fact, the results of our partial correlation results suggest that both neuronal death and birth play a role the 
structural integrity of the hippocampus. Hence, age-related neuronal atrophy does not fully explain decrease in hippocampal volume with age. What does seem to be more related to and better explains the age-related reduction in volume in both old and middle-aged rats is the rate of neurogenesis in the hippocampus.

Although the volume of the hippocampus, as observed by MRI, decreases beginning sometime in adulthood, the functional metabolic integrity as measured by MRS stays preserved in the cells that remain in old age. Together, these results suggest that the structural integrity of the aged hippocampus is compromised but the functional metabolic integrity of neurons that remain is preserved. This finding, however, stands in stark contrast to consistent reports of alterations in levels of different metabolites in the aged human hippocampus (e.g., Driscoll et al., 2003a; Schuff et al., 1999). Whereas MRS is now routinely employed in human research, it is still a fairly new modality of MR and this is the first attempt to evaluate the metabolic profile of the aged rat hippocampus employing this methodology. Hence, we are unsure as to why this discrepancy between rat and human findings exists.

A couple of possibilities are apparent. One, there could be a clear cross-species difference, although it is hard to speculate what the source of this difference might be given that thus far magnetic resonance spectroscopy has not been employed to study normally aging rats. When employed to study metabolic profiles in the rat brain after ischemia, for example, (Demougeot et al., 2001), 
the findings are similar to those reported in humans. The possibility remains that the metabolic processes in the senescent rat brains are fundamentally different from those occurring in the injured brain or in the aging human brain for that matter. Second, as previously mentioned in Experiment 2, in addition to possible differences between species, the rat strain differences are mentioned as a possible explanation for differential findings related to synaptic plasticity, and the same could be the case in relation to detecting metabolic age-related differences in the rat brain. Given that this is the first MRS study of the normally aging rat, both possibilities of cross-species and strain differences contributing to the discrepancy in the results remain to be further investigated. In addition, in regard to MRS, the lack of findings in the aged rat hippocampus, although preliminary, does bring into question the interpretations of cognitive deficits that accompany NAA reduction in human aging.

Overall, a pattern of relationships emerged from the measures of hippocampal integrity that were obtained on several different levels of investigation. More specifically, we found a significant relationship between performances on two hippocampus-dependent tasks, one spatial and one non-spatial in nature, a relationship that remained even when the age was controlled for. Further, performance on both hippocampus-dependent tasks was significantly correlated with hippocampal volume measured in the same rats in vivo. The abovementioned patterns of relationships resembles those previously reported in humans in relation to aging (Driscoll et al., 2003a). 
In addition, significant relationships emerged between the hippocampusdependent learning and memory and the number of DCX-labeled cells and BrdU/NeuN-labeled cells respectively, but not to the same degree with Ki67labeled cells. Recent studies that have attempted to correlate neurogenesis with spatial performance report a reduction in both neurogenesis and behavioral impairments in the context of aging, but fail to detect a clear relationship between the two (Bizon et al., 2004; Merrill et al., 2003). These studies, however, have primarily assessed the rate of cell proliferation. Thus, it has been suggested that survival, rather than the proliferation, is more important when it comes to contributing to hippocampus-dependent functioning (Drapeau et al., 2003). Our findings are in line with this idea, given the relationships between the measures of neuronal differentiation and survival with behavior. They are not independent of the reduction in the proliferation rate. Given that the rate of proliferation was severely reduced in the aged rats to start with, the age-related differences in the rate of neuronal differentiation and survival could be seen as a direct consequence of reduced proliferation. Such observations make sense. The cells that are proliferating are yet to migrate to their final destination and are not expected to have yet committed to neuronal faith or otherwise, hence are not functionally incorporated into the existing circuitry.

With regard to ${ }^{1} \mathrm{H}$ MRS, even though we found no age-related alterations in the hippocampal metabolite levels, namely NAA, the lack of results is not necessarily uninteresting. To the best of our knowledge, this is the first attempt to examine the aging rat brain with MRS and our results are preliminary. Unfortunately, the 
lengthy scan time in order to acquire structural images and in order to obtain quality spectra from the hippocampi precluded us from examining other regions of the brain with same methodology. It would certainly be interesting to know whether alterations in the metabolic profile are present in any other regions of the aged rat brain, or if this strain of rat may be immune to metabolic alterations with age.

Potentially more interesting, at least to the MRS community, is our investigation of neuronal and mitochondrial numbers in an attempt to better explain what NAA levels are representing. Given that hippocampal NAA levels remain stable in the face of aging but neuronal count was reduced in the old group, we have to dismiss the view of NAA as a putative neuronal marker or more precisely as an indicator of neuronal atrophy or neuronal density. Given that the number of mitochondria also remained stable with age, our results lead us to agree with more recent propositions of NAA as a marker of neuronal viability or neuronal metabolic function.

Together, our results define some changes that occur in the rat hippocampus during the process of normal aging and suggest FBNF1 rats to be a very useful model of normal aging in humans. The pattern of similarities in changes that occur with aging in both rats and humans alike is striking, with the exception of MRS results. Also, we have not only validated learning and memory tasks that are hippocampus-dependent, sensitive to the aging process, and provide a reasonable basis for cross-species comparisons, but we have also affirmed 
methodology (MRI and MRS) that can be readily applied in a similar fashion to study both humans and rodents. In addition, we have described some alterations that occur in the hippocampus with normal aging that seem to be related to the hippocampus-dependent learning and memory dysfunction.

Even though some of these alterations begin quite early in adulthood they should be viewed as a part of normal aging process and as distinct from agerelated pathology. It is reasonable to consider that such changes can be of adaptive nature to an aging organism, even though the alterations are at times substantial. If we buy into this argument, then it would reasonable to assume that both elderly humans and rats are able to remain functional, and get along in and adapt to their environment despite the age-related changes they might be experiencing. The difficulty arises, however, in detecting and describing the point at which normal aging crosses over to pathology and an organism suffers a substantial loss in the quality of life. It is the identification of this point that will ultimately allow us to better target our diagnosis, prevention, and treatment efforts directed toward alleviating the devastation that accompanies the agerelated pathology. 


\section{Closing Comments}

Cognitive outcomes resulting from old age are said to vary in frequency, direction, and especially extent of the deficits. For example, a slowing of function, physical and psychological, is universal in people over the age of 60 , but there is also a good chance of reaching a very old age with no notable memory problems for example. Also, some cognitive processes are not very susceptible to the deleterious effects of age, and some (such as vocabulary) even improve with age. Similarly, even though $\varepsilon 4$ allele of the APOE constitutes a genetic risk for $A D$, not all $\varepsilon 4$ allele carriers develop the disease. Interestingly, the fact in animal aging literature is that about a third of aged animals show no memory impairment and perform on memory tests as well as their younger counterparts (Gallagher and Rapp, 1997).

One aspect of cognitive aging that has been largely ignored is the influence of genetic factors on the expression of cognitive phenotypes. A current theory concerning the etiology of age-related cognitive decline takes a combination of co-factors into an account (McDonald, 2002). This theory hints at the underlying genetic factors as well as individual differences that may differentially affect the aging process and its outcomes. This theory divides co-factors into two categories, a passive factor category (such as senile plaques, neurofibrillary tangles, head trauma, cholinergic depletion, and prolonged glucocorticoid exposure), and active factors (such as seizures, vascular abnormalities, circadian dysfunction, and other unknown environmental influences unique to 
each organism). The idea is that the more factors that are present from each category the larger the damage to the hippocampus at a faster rate, and more pronounced and severe the deficits. The type, number, and combination of cofactors vary from person to person (or rather rat to rat), and could possibly account for individual differences in cognitive aging.

Laboratory rodents are especially suitable as models of cognitive aging in humans because they have a relatively short lifespan and because many tests have been developed to assay their cognitive performance. Animal models may be helpful for gaining insight into normal aging and for determining the relative contribution of various factors to age-related changes. Changes occurring with age might be due to extrinsic factors (ecology, environment) or intrinsic factors (genetics), or the combination of both. In laboratory experiments extrinsic factors are normally well controlled for and standardized in order to eliminate them as sources of possible confounding effects and also allow for intrinsic factors to take effect.

Both human and non-human animal studies are needed in order to further our understanding of the genetic and environmental effects as well as the processes that may be influencing variability in behavior and its functional outcome with aging. Future research should be directed at elucidating the nature and role of genes on behavior, and on genetic-environmental interplay. 


\section{Conclusion}

A recent revolution in neuroscience techniques has lead to an accumulation of converging evidence on neural, physiological, and cognitive changes with aging. The substrates of the age-related cognitive deficits are still unclear. Spatial memory in particular has been extensively studied in aged rodents, but not as well in aged humans because techniques for investigating brain-behavior relations in vivo were not well developed. Recent advancement in magnetic resonance has allowed visualization of hippocampal anatomy and quantification of changes in vivo. Such techniques, however, not only allow us to delineate the neuroanatomical substrates in health and disease, but are also applicable to both humans and non-human animals allowing for direct cross-species comparisons.

The aging rat as a model for human aging may shed light on the aging process and the accompanying neurodegenerative disorders. A particular advantage of rodent aging studies is that the contribution of genetic and environmental factors can be investigated and strictly controlled. The newly developed transgenic mouse models promise to further unravel the genetic factors underlying aging (Vijg, 2000). It has been suggested that the age-related cognitive impairments are related to a decline in hippocampal plasticity, which in turn may dependent on alterations in gene expressions. The molecular products of genomic responses may lead to permanent biological and/or morphological 
changes in the brain. Further inquiry is needed to identify the factors responsible for the susceptibility of this structure to aging.

An important feature of animal models of aging and animal models in general, is that they closely mimic the characteristics of human disorders and that they are sensitive to individual differences. We have not investigated individual differences in the current study, as much larger number of animals would be required to be able to sufficiently detect such differences. At the same time we feel that our rat model of human aging does closely mimic the characteristics of human aging and will prove to be very useful in studying both normal and pathological aging in the future, especially because the tasks and methodology employed will allow for cross-species comparisons.

Many intervening variables must be considered when evaluating the usefulness of tasks to study the effect of age on learning and memory. For example, one should insure that performance of both human and non-human animals is not influenced by sensory or motor deficits, or differences in motivational or problem-solving strategies. The goal for the future is to develop more sensitive animal and human behavioral tasks that would permit an evaluation of similar processes in both human and non-human animals and that would also allow for an evaluation of the underlying neurobiology on cognitive processes in a tractable manner. 
Although many questions regarding the navigation network as well as the functional role of the hippocampus remain to be determined, the behavioral tasks described here may be the closest link yet in behavioral tasks implemented between humans and other mammals. Such research may not only aid in better understanding of the aging processes, but will also further our overall understanding of the role of the hippocampus in learning and memory. 


\section{References}

Aberg MAI, Aberg ND, Hedbacker H, Oscarsson J, Eriksson PS. Peripheral infusion of IGF-I selectively induces neurogenesis in the adult rat hippocampus. J Neurosci 2000; 20:2896-2903.

Aguirre GK, Detre JA, Alsop DC, DEsposito M. The parahippocampus subserves topographical learning in man. Cereb Cortex 1996; 6:823-829.

Allegrini PR, Sauer D. Application of magnetic resonance imaging to the measurement of neurodegeneration in rat brain: MRI data correlate strongly with histology and enzymatic analysis. Magn Reson Imaging 1992; 10:773-778.

Altman J, Das GD. Autoradiographic and histological evidence of postnatal neurogenesis in rats. J Comput Neurol 1965; 124:319-335.

Alvarado MC, Rudy JW. Some properties of configural learning: An investigation of the transverse patterning problem. J Exp Psychol Anim Behav Proc 1992; 18:145-153.

Alvarado MC, Rudy JW. Rats with damage to the hippocampal formation are impaired on the transverse-patterning problem but not on elemental discrimination. Behav Neurosci 1995a; 109:204-211.

Alvarado MC, Rudy JW. A comparison of kainic acid plus colchicine and ibotenic acid-induced hippocampal formation damage on four configural tasks in rats. Behav Neurosci

1995b; 109:1052-1062.

Amaral DG, Witter MP. The three-dimensional organization of the hippocampal formation: A review of anatomical data. Neurosci 1989; 31:571-591.

Amaral DG, Witter MP. The hippocampal formation. In: Paxinos G, ed. The Rat Nervous System, $2^{\text {nd }}$ ed. New York: Academic Press, 1995; 443-493.

Anagnostaras SG, Maren S, Sage JR, Goodrich S, Fanselow MS. Temporally graded retrograde amnesia of contextual fear after hippocampal damage in rats: Within-subjects examination. J Neurosci 1999; 19:1106-1114.

Assaf Y, Beit-Yannai E, Shohami E, Berman E, Cohen Y. Diffusion and T2weighted MRI of closed head injury in rats: a time course study and correlation with histology. Magn Reson Imaging 1997; 15:77-85.

Astur RS, Constable RT. Hippocampal dampening during a relational memory task. Behav Neurosci 2004; 118:667-675. 
Astur RS, Ortiz ML, Sutherland RJ. A characterization of performance by men and women in a virtual Morris water task: a large and reliable sex difference. Behav Brain Res 1998a; 93:185-190.

Astur RS, Sutherland RJ. Configural learning in humans: The transverse patterning problem. Psychobiology 1998b; 26:176-182.

Astur RS, Taylor LB, Mamelak AN, Philpott L, Sutherland RJ. Humans with hippocampal damage display severe spatial memory impairments in a virtual Morris water task. Beh Brain Res 2002; 132:77-84.

Astur RS, Tropp J, Sava S, Constable RT, Markus EJ. Sex differences and correlations in a virtual Morris water task, a virtual radial arm maze, and mental rotations. Behav Brain Res 2004; 151:103-115.

Baba T, Moriguchi M, Natori $Y$, Katsuki C, Inoue T, Fukui M. Magnetic resonance imaging of experimental rat brain tumors: histopathological evaluation. Surg Neurol 1990; 34:378-382.

Bach ME, Barad M, Son H, Zhuo M, Lu YF, Shih R, Mansuy I, Hawkins RD, Kandel ER. Age-related defects in spatial memory are correlated with defects in the late phase of hippocampal long-term potentiation in vitro and are attenuated by drugs that enhance the CAMP signaling pathway. Proc Natl Acad Sci USA $1999 ; 96: 5280-5285$

Bannerman DM, Yee BK, Lemaire M, Wilbrecht L, Jarrard L, Iverson SD, Rawlins JN, Good MA. The role of entorhinal cortex in two forms of spatial learning and memory. Exp Brain Res 2001; 141:281-303.

Barnea A, Nottebohm F. Seasonal recruitment of hippocampal neurons in adult free-ranging black-capped chickades. Proc Natl Acad Sci USA 1994; 8:1121711221.

Barnes CA. Memory deficits associated with senescence: A neurophysiological and behavioral study in the rat. J Comp Physiol Psychol 1979; 93:74-104.

Barnes CA. Aging and the physiology of spatial memory. Neurobiol Aging 1988; 9:563-570.

Barnes CA. Long-term potentiation and the ageing brain. Philos Trans $\mathrm{R}$ Soc Lond B Biol Sci 2003; 358:765-772.

Barnes CA, Eppich C, and Rao G. Selective improvement of aged rat short-term memory by 3, 4-diaminopyridine. Neurobiol Aging 1989; 10:337-341.

Barnes $C A$, Markowska $A L$, Ingram DK, Kametani $H$, Spangler EL, Lemken VJ, and Olton DS. Acetyl-I-carnitine 2: Effects on learning and memory performance of aged rats in simple and complex mazes. Neurobiol Aging 1990; 11:499-506. 
Barnes CA, MCNaughton BL. Physiological compensation for loss of afferent synapses in rat hippocampal granule cells during senescence. J Physiol - London $1980 ; 309: 473-485$.

Barnes $C A$, McNaughton $B L$. An age comparison of the rates of acquisition and forgetting of spatial information in long-term enhancement of hippocampal synapses. Behav Neurosci 1985; 99:1040-1048.

Barnes CA, Rao G, Houston FP. LTP induction threshold change in old rats at the perforant path-granule cell synapse. Neurobiol Aging 2000; 21:613-620.

Barnes CA, Suster MS, Shen J, McNaughton BL. Multistability of cognitive maps in the hippocampus of old rats. Nature $1997 ; 388: 272-275$.

Bates TE, Strangward JK, Davey GP, Munro PMG, Clark JB. Inhibition of Nacetylaspartate production: implications for ${ }^{1} \mathrm{H}$ MRS studies in vivo. Neuroreport $1996 ; 7: 1397-1400$.

Bayer SA. Changes in the total number of dentate granule cells in juvenile and adult rats: a correlated volumetric and $3 \mathrm{H}$-thymidine autoradiographic study. Exp Brain Res 1982; 46:315-323.

Ben Horin N, Hazvi S, Bendel P, Shul R. The ontogeny of a neurotoxic lesion in rat brain revealed by combined MRI and histology. Brain Res 1996; 718:97-104.

Benuch M, D'Adamo AF. Acetyl transport mechanism. Biochem Biophys Acta $1968 ; 152: 611-618$.

Berch DB, Israel M. Solution of the transverse patterning problem: Response to cue-cue relations. Psychon Sci 1971; 23:383-384.

Birken DL, Olendorf WH. N-acetyl-L-aspartic acid - a literature review of a compound prominent in $\mathrm{H}-1-\mathrm{NMR}$ spectroscopic studies of brain. Neurosci Biobehav Rev 1989; 13:23-31.

Bizon JL, Lee HJ, Gallagher M. Neurogenesis in a rat model of age-related cognitive decline. Aging Cell 2004; 3:227-234.

Bliss TVP, Gardner-Medwin AR. Long-lasting potentiation of synaptic transmission in the dentate area of the unanesthetised rabbit following stimulation of the perforant path. J Physiol (London) 1973; 232:357-374.

Bliss TVP, Lomo T. Long-lasting potentiation of synaptic transmission in the dentate area of the anesthetized rabbit following stimulus of perforant path. J Physiol (London) 1973; 232:331-356. 
Bohbot V, Kalina M, Stepankova J, Spackova N, Petrides M, Nadel L. Spatial memory deficits in patients with lesions to the right hippocampus and to the right parahippocampal cortex. Neuropsychologia 1998; 36:1217-38.

Bouilleret V, Nehlig A, Marescaux C, Namer IJ. Magnetic resonance imaging follow-up of progressive hippocampal changes in a mouse model of mesial temporal lobe epilepsy. Epilepsia 2000; 41:642-650.

Brooks WM, Sabet A, Sibbitt WL, Barker PB, van Zijl P, Moonen C, Duyn J. Neurochemical quantification in Systemic Lupus Erythematosus. J Rheumatol $1997 ; 24: 2323-2329$

Brooks WM, Jung RE, Ford CC, Greinel EJ, Sibbitt WL. Relationship between neurometabolite derangement and neurocognitive dysfunction in systemic lupus erythematosus. J Rheumatol 1999; 26:81-85.

Bussey TJ, Warburton EC, Aggleton JP, Muir JL. Fornix lesions can facilitate acquisition of the transverse patterning task: A challenge for "configural" theories of hippocampal function. J Neurosci 1998; 18:1622-1631.

Cameron HA, McKay RDG. Adult neurogenesis produces a large pool of new granule cells in the dentate gyrus. J Comp Neurol 2001; 435:406-407.

Cameron HA, Wooley CS, McEwen BS, Gould E. Differentiation of newly born neurons and glia in the dentate gyrus of the adult rat. Neuroscience 1993; $56: 337-344$

Carlen M, Cassidy RM, Brismar H, Smith GA, Enquist LW, Frisen J. Functional integration of adult-born neurons. Curr Biol 2002; 12:606-608.

Chang L, Ernst T, Poland RE, Jenden DJ. In vivo proton magnetic resonance spectroscopy of the normal aging human brain. Life Sci 1996; 58:2049-2056.

Cho $\mathrm{YH}$, Friedman $E$, Silva AJ. Ibotenate lesions of the hippocampus impair spatial learning but not contextual fear conditioning in mice. Behav Brain Res 1999; $98: 77-87$.

Cipolotti L, Shallice T, Chan D, Fox N, Scahill R, Harrison G, Stevens J, Rudge P. Long-term retrograde amnesia ...the crucial role of the hippocampus. Neuropsychologia 2001; 39:151-172.

Clark RE, Broadbent NJ, Squire LR. Hippocampus and remote spatial memory in rats. Hippocampus in press.

Clark RE, Broadbent NJ, Zola SM, Squire LR. Anterograde amnesia and temporally graded retrograde amnesia for a non-spatial memory task after lesions of hippocampus and subiculum. J Neurosci 2002; 22:4663-4669. 
Colombo M, Fernandez T, Nakamura K, Gross CG. Functional differentiation along the anterior-posterior axis of the hippocampus in monkeys. J Neurophysiol 1998; 80:1002-1005.

Corkin S. Lasting consequences of bilateral medial temporal lobectomy: Clinical course and experimental findings in H.M. Semin Neurol 1984; 4:249-259.

Curtis MA, Penney EB, Pearson AG, van Roon-Mom WMC, Butterworth NJ, Dragunow $M$, Connor $B$, Faull RLM. Increased cell proliferation and neurogenesis in the adult human Huntington's disease brain. Proc Natl Acad Sci USA 2003; $100: 9023-9027$.

D'Adamo AF, Smith JC, Woiler C. The occurrence of N-acetylaspartate amidohidolase (Aminoacylase II) in the developing rat. J Neurochem1973; $20: 1275-1278$.

Dautry C, Vaufrey F, Brouillet E, Bizat N, Henry PG, Cone F, Bloch G, Hantraye $\mathrm{P}$. Early $\mathrm{N}$-Acetylaspartate depletion is a marker of neuronal dysfunction in rats and primates chronically treated with the mitochondrial toxin 3-Nitropropionic acid. J Cereb Blood Flow Metabol 2000; 20:789-799.

Davie CA, Hawkins CP, Barker G], Brennan A, Tofts PS, Miller DH, McDonald WI. Serial proton magnetic resonance spectroscopy in acute multiple sclerosis lesions. Brain 1994; 117:49-58.

De Stefano N, Matthews PM, \& Arnold DL. Reversible decrease in Nacetylaspartate after acute brain injury. Mag Res Med 1995; 34:721-727.

de Toledo-Morrell L, Morrell F. Electrophysiological markers of aging and memory loss in rats. Ann NY Acad Sci 1985; 444:296-311.

de Toledo-Morrell L, Geinisman Y, Morrell F. Age-dependent alterations in hippocampal synaptic plasticity: Relation to memory disorders. Neurobiol Aging $1988 ; 9: 581-590$

Demougeot C, Garnier P, Mossiat C, Bertrand N, Giroud M, Beley A, Marie C. Nacetylaspartate, a marker of both cellular dysfunction and neuronal loss: its relevance to studies of acute brain injury. J Neurochem 2001; 77:408-415.

Derrick $B E$, York $A D$, Martinez $J L J$ r. Increased granulle cell neurogenesis in the adult dentate gyrus following mossy fiber stimulation sufficient to induce longterm potentiation. Brain Res 2000; 857:300-307.

Diana G, Domenici MR, Loizzo A, Scotti de Carolis A, Sagratella S. Age and strain differences in rat place learning and hippocampal dentate gyrus frequencypotentiation. Neurosci Lett 1994a; 171:113-116. 
Diana G, Scotti de Carolis A, Frank C, Domenici MR, Sagratella S. Selective reduction of hippocampal dentate frequency potentiation in aged rats with impaired place learning. Brain Res Bull 1994b; 35:107-111.

Douglas RM, Goddard GV. Long-term potentiation of the perforant path-granule cell synapse in the rat hippocampus. Brain Res $1975 ; 86: 205-215$.

Drapeau E, Mayo W, Aurousseau C, Le Moal M, Piazza PV, Abrous DN. Spatial memory performances of aged rats in the water task predict levels of hippocampal neurogenesis. Proc Natl Acad Sci USA 2003; 100:14385-14390.

Driscoll I, Epp J, Hamilton DA, Sutherland RJ. Transverse patterning discrimination problem: Configural learning in the young, middle-aged, and elderly adults. $32^{\text {nd }}$ Annual Meeting of the International Neuropsychological Society Abstract, 2004a.

Driscoll I, Hamilton DA, Petropoulos H, Yeo RA, Brooks WM, Sutherland RJ. The aging hippocampus: cognitive, biochemical, and structural findings. Cereb Cortex 2003a; 13:1344-1351.

Driscoll I, Hamilton DA, Yeo RA, Brooks WM, Sutherland RJ. Virtual navigation in humans: the impact of age, sex, and hormones on place learning. Horm Behav $2005 ; 427: 326-335$.

Driscoll I, Yeo RA, Petropoulos H, Brooks WM, Sutherland RJ. The polymorphism of Apolipoprotein $E$ in normal aging: Behavioral, biochemical, and structural measures of the hippocampus. Society for Neuroscience Abstract, vol. 29, 2003b.

Driscoll I, Sutherland RJ, Prusky GT, Rudy JW. Damage to the hippocampal formation does not disrupt representational flexibility as measured by a novelty transfer task. Behav Neurosci 2004b; 118:1427-1432.

Dusek JA, Eichenbaum $H$. The hippocampus and transverse patterning guided by olfactory cues. Behav Neurosci 1998; 112:762-771.

Eichenbaum $\mathrm{H}$. The hippocampal system and declarative memory in animals. J Cogn Neurosci 1992; 4:217-231.

Eichenbaum $H$. The hippocampus and declarative memory: cognitive mechanisms and neural codes. Behav Brain Res 2001; 11:709-711.

Ende GR, Laxer KD, Knowlton RC, Matson GB, Schuff N, Fein G, Weiner MW. Temporal lobe epilepsy: bilateral hippocampal metabolite changes revealed at proton MR spectroscopic imaging. Radiology 1997; 202:809-817. 
Eriksson PS, Perfilieva E, Bjork-Eriksson T, Alborn AM, Nordborg C, Peterson DA, Gage FH. Neurogenesis in the adult human hippocampus. Nature Med 1998; 4:1313-1317.

Esiri M. Dementia and normal aging: neuropathology. In: Huppert FA, Brayne C, O'Connor DW, eds. Dementia and normal aging. Cambridge, UK: Cambridge University Press, 1994; 385-429.

Evans DA, Beckett LA, Field TS, Feng L, Albert MS, Bennett DA, Tycko B, Mayeux R. Apolpoprotein E epsilon 4 and incidence of Alzheimer's Disease in a community population of older people. J A Med Assoc 1997; 277:822-824.

Fanselow MS. Learning theory and neuropsychology: Configuring their disparate elements in the hippocampus. J Exp Psychol - An Behav Proces 1999; 25:275283.

Fanselow MS. Contextual fear, gestalt memories, and the hippocampus. Behav Brain Res 2000; 110:73-81.

Frank MJ, Rudy JW, O'Reilly RC. Transitivity, Flexibility, Conjunctive Representations, and the Hippocampus. II. A Computational Analysis. Hippocampus 2003; 13:299-312.

Frankland PW, Cestari V, Filipkowski RK, MCDonald R, Silva A. The dorsal hippocampus is essential for context discrimination, but not for contextual conditioning. Behav Neurosci 1998; 112:863-875.

Frick KM, Baxter MG, Markowska AL, Olton DS, Price DL. Age-related spatial reference and working memory deficits assessed in the water task. Neurobiol Aging 1995; 16:149-160.

Fukuzako H, Hashiguchi T, Sakamoto Y, Okamura H, Doi W, Takenouchi K, Takigawa M. Metabolite changes with age measured by proton magnetic resonance spectroscopy in normal subjects. Psychiatry Clin Neurosci 1997; $51: 261-263$.

Gaffan D. Scene specific memory for objects - a model of episodic memory impairment in monkeys with fornix transection. J Cogn Neurosci 1994; 6:305320.

Gage FH, Chen KS, Buszaki G, \& Armstrong D. Experimental approaches to agerelated cognitive impairments. Neurobiol Aging 1988; 9:645-655.

Gage $\mathrm{FH}$, Dunnett SB, Bjorklund A. Spatial learning and motor deficits in aged rats. Neurobiol Aging 1984; 5:43-48.

Gallagher M, Bostock E, King R. Effects of opiate antagonists on spatial memory in young and aged rats. Behav Neural Biol 1985; 44:374-385. 
Gallagher M, Burwell R, Burchinal M. Severity of spatial impairment in aging: Development of a learning index for performance in the Morris water task. Behav Neurosci 1993; 107:618-626.

Gallagher M, Holland PC. Preserved configural learning and spatial-learning impairment in rats with hippocampal damage. Hippocampus 1992; 2:81-88.

Gallagher M, Pelleymounter MA. Spatial learning deficits in old rats: A model for memory deficits in the aged. Neurobiol Aging 1988; 9:549-556.

Gallagher M, Rapp PR. The use of animal models to study the effects of aging on cognition. Ann Rev Psychol 1997; 48:339-370.

Garry PJ, Goodwin JS, Hunt WC, Hooper EM, Leonard AG. Nutritional status in healthy elderly population: Dietary and supplemental intakes. Am J Clin Nutr $1982 ; 36: 319-331$.

Garry PJ, Hunt WC, Koehler KM, VanderJagt DJ, Vellas BJ. Longitudinal study of dietary intakes and plasma lipids in healthy elderly men and women. Am J Clin Nutr 1992; 55:682-688.

Geinisman $Y$, deToledo-Morrell L, Morrell F, Heller RE. Hippocampal markers of age-related memory dysfunction: behavioral, electrophysiological and morphological perspectives. Prog Neurobiol 1995; 45:223-252.

Gianoulakis C. Rats exposed prenatally to alcohol exhibit impairment in spatial navigation test. Behav Brain Res 1990; 36:217-228.

Goldman SA. Adult neurogenesis: From canaries to the clinic. I Neurobiol 1998; $36: 267-286$

Golomb J, de Leon MJ, Kluger A, George AE, Tarshish C, Ferris SH. Hippocampal atrophy in normal aging: An association with recent memory impairment. Arch Neurol 1993; 50:967-973.

Gould E, Beylin A, Tanapat P, Reeves AJ, Shors TJ. Hippocampal-dependent learning enhances the survival of granule neurons generated in the dentate gyrus of adult rats. Integrat Physiol Behav Sci 1999a; 34:120-121.

Gould E, MCEwen BS, Tanapat P, Galea LAM, Fuchs E. Neurogenesis in the dentate gyrus of the adult three shrew is regulated by psychosocial stress and NMDA receptor activation. J Neurosci 1997; 17:2492-2498.

Gould E, Reeves AJ, Fallah M, Tanapat P, Gross CG, Fuchs E. Hippocampal neurogenesis in Old World primates. Proc Natl Acad Sci USA 1999b; 96:52635267. 
Gould E, Tanapat P, Hastings NB, Shors TJ. Neurogenesis in adulthood: a possible role in learning. Trends Cogn Neurosci 1999c; 3:186-192.

Gould E, Tanapat P, McEwen BS, Flugge G, Fuchs E. Proliferation of granule cell precursors in the dentate gyrus of adult monkeys is diminished by stress. Proc Natl Acad Sci USA 1998; 95:3168-3171.

Griffiths DP, Clayton NS. Testing episodic memory in animals: A new approach. Physiol Behav 2001; 73:755-762.

Hamilton DA, Sutherland RJ. Blocking in human place learning: evidence from virtual navigation. Psychobiology 1999; 27:453-461.

Hamilton DA, Driscoll I, Sutherland RJ. Human place learning in a virtual Morris water task: some important constraints on the flexibility of place navigation. Behav Brain Res 2002; 129:159-170.

Hamilton DA, Kodituwakku P, Sutherland RJ, and Savage DD. Children with Fetal Alcohol Syndrome are impaired at place learning but not cued-navigation in a virtual Morris water task. Behav Brain Res 2003; 143:85-94.

Haughey N], Nath A, Chan St, Borchard AC, Rao MS, Mattson MP. Disruption of neurogenesis by amyloid beta-peptide, and perturbed neural progenitor cell homeostasis, in models of Alzheimer's disease. J Neurochem 2002; 83:15091524.

Hebda-Bauer EK, Morano MI, Therrien B. Aging and corticosterone injections affect spatial learning in Fischer-344 x Brown Norway rats. Brain Res 1999; 827:93-103.

Hirsch R. The hippocampus and contextual retrieval of information from memory. A theory. Behav Biol 1974; 12, 421-444.

Hugg JW, Kuzniecky RI, Gilliam FG, Morawetz RB, Fraught RE, Hetherington HP. Normalization of contralateral metabolic function following temporal lobectomy demonstrated by ${ }^{1} \mathrm{H}$ magnetic resonance spectroscopic imaging. Ann Neurol $1996 ; 40: 236-239$.

Jacobs WJ, Thomas KGF, Laurance HE, Nadel L. Place learning in virtual space II. Topographical relations as one dimension of stimulus control. Learn Motiv $1998 ; 29: 288-308$.

Jarrard LE. Selective hippocampal lesions and behavior: Effects of kainic acid lesions on performance of place and cue tasks. Behav Neurosci 1983; 97:873889.

Jenkins BG, Klivenyi $P$, Kustermann $E$, Andreassen OA, Ferrante RJ, Rosen BR, Beal MF. Nonlinear decrease over time in $\mathrm{N}$-acetyl aspartate levels in the 
absence of neuronal loss and increases in glutamine and glucose in transgenic Huntington's disease mice. J Neurochem 2000; 74:2108-2119.

Jernigan TA, Archibald SL, Berhow MT, Sowell ER, Foster DS, Hesselink JR. Cerebral structure on MRI. Part I: Localization of age related changes. Biol Psychiatry 1991; 29:55-67.

Jessberger S, Kempermann G. Adult-born hippocampal neurons mature into activity-dependent responsiveness. Eur J Neurosci 2003; 18:2707-2712.

Jin KL, Peel AL, Mao XO, Xie L, Cottrell BA, Henshall DC Greenberg DA. Increased hippocampal neurogenesis in Alzheimer's disease. Proc Natl Acad Sci USA 2004; 101:343-347.

Jin $\mathrm{KL}$, Sun $\mathrm{YJ}$, Xie L, Batteur S, Mao XO, Smelick C, Logvinova A, Greenberg DA. Neurogenesis and aging: FGF-2 and HB-EGF restore neurogenesis in hippocampus and subventricular zone of aged mice. Aging Cell 2003; 2:175183.

Jung RE, Brooks WM, Yeo RA, Chiulli SJ, Weers DC, Sibbitt WL. Biochemical markers of intelligence: a proton MR study of normal human brain. Proc R SOC Lond B Biol Sci 1999; 266:1375-1379.

Jung RE, Chiulli SJ, Friedman SD, Brooks WM., Yeo RA. Magnetic Resonance Spectroscopy of brain $\mathrm{N}$-acetylaspartate predicts neuropsychological performance following traumatic brain injury. Arch Clin Neuropsychology 1998; $13: 144-144$

Kalra S, Cashman N, Genge A, Arnold D. Recovery of N-acetylaspartate in corticomotor neurons of patients with ALS after riluzole therapy. Neuroreport $1998 ; 9: 1757-1761$.

Kaplan MS, Hinds JW. Neurogenesis in the adult rat: electron microscopic analysis of light radioautographs. Science 1977; 197:1092-1094.

Kempermann G, Gast D, Gage FH. Neuroplasticity in old age: Sustained fivefold induction of hippocampal neurogenesis by long-term environmental enrichment. Ann Neurol 2002; 52:135-143.

Kempermann G, Kuhn HG, Gage FH. More hippocampal neurons in adult mice living in an enriched environment. Nature 1997; 386:493-495.

Kempermann G, Kuhn HG, Gage FH. Experience-induced neurogenesis in the senescent dentate gyrus. 3 Neurosci 1998; 18:3206-3212.

Kempermann G, Gast D, Gage, FH. Neuroplasticity in old age: Sustained fivefold induction of hippocampal neurogenesis by long-term environmental enrichment. Ann Neurol 2002; 52:135-143. 
Kempermann G, Wiskott L, Gage FH. Functional significance of adult neurogenesis. Cur Op Neurobiol 2004; 14:186-191.

Kirasic KC, Allen GC. Aging, spatial performance and spatial competence. In: Clarness N, ed. Aging and Human Performance. New York: Wiley, 1985; 191224.

Kleim JA, Barbay S, Cooper NR, Hogg TM, Reidel CN, Remple MS, Nudo RJ. Motor learning-dependent synaptogenesis is localized to functionally reorganized motor cortex. Neurobiol Learn Mem 1996; 77: 63-77.

Kornack DR, Rakic P. Continuation of neurogenesis in the hippocampus of the adult macaque monkey. Proc Natl Acad Sci USA 1999; 96:5768-5773.

Kuhn HG, Dickinson-Anson H, Gage FH. Neurogenesis in the dentate gyrus of the adult rat: age-related decrease of neuronal progenitor proliferation. J Neurosci 1996; 16:2027-2033.

Laurance HE, Thomas KGF, Newman KC, Kaszniak AW, Nadel L, Jacobs JW. Older adults map novel environments but do not place learn: Findings from a computerized spatial task. Aging Neuropsychol Cog 2002; 9:85-97.

Lee $B$, English JA, Paul IA. LP-BM5 infection impairs spatial working memory in C57BL/6 mice in the Morris water task. Brain Res 2000; 856:129-134.

Lie DC, Song H, Colamarion SA, Ming G, Gage FH. Neurogenesis in the adult brain: New strategies for central nervous system diseases. Annu Rev Pharmacol Toxicol 2004; 44:39-421.

Lindner MD, Gribkoff VK. Relationship between performance in the Morris water task, visual acuity, and thermoregulatory function in aged F-344 rats. Beh Brain Res $1991 ; 45: 45-55$.

Lipton SA. Immune activation and neuronal injury in AIDS: Reply. Trends Neurosci 1992; 15:75-79.

Lynch MA, Voss KL. Membrane arachidonic acid concentration correlates with age and induction of long-term potentiation in the dentate gyrus of the rat. Eur J Neuroscì 1994; 6:1008-1014.

Mackintosh NJ. A theory of attention: Variation in the associabilty of stimuli with reinforcement. Psychol Rev 1975: 82:276-298.

Maguire EA, Burgess N, Donnett JG, Frackowiak RSJ, Frith CD, O'Keefe J. Knowing where and getting there: A human navigation network. Science 1998; 280:921-924. 
Maguire EA, Burgess N, O'Keefe J. Human spatial navigation: cognitive maps, sexual dimorphism, and neural substrates. Curr Opin Neurobiol 1999; 9:171177.

Maguire EA, Frackowiak RS, Frith CD. Learning to find your way - a role for the human hippocampal region. Proc R Soc Lond B Biol Sci 1996; 263:1745-50.

Maguire EA, Frackowiak RS, Frith CD. Recalling routes around London: activation of the right hippocampus in the taxi drivers. I Neurosci 1997; 17:7103-10.

Maguire EA, Gadian DG, Johnsrude IS, Good CD, Ashburner J, Frackowiak RS, \& Frith $C D$. Navigation-related structural change in the hippocampi of taxi drivers. Proc Natl Acad Sci USA 2000; 97:4398-403.

Maren S. Neurobiology of Pavlovian fear conditioning. Ann Rev Neurosci 2001; 24:87-931.

Maren S, Aharonov G, Fanselow MS. Neurotoxic lesions of the dorsal hippocampus and Pavlovian fear conditioning in rats. Beh Brain Res 1997; $88: 261-274$

Martin SJ, Grimwood PD, Morris RGM. Synaptic plasticity and memory: An evaluation of the hypothesis. Ann Rev Neurosci 2000; 23:649-711.

Markowska AL. Sex dimophism in the rate of age-related decline in spatial memory: relevance to alterations in the estrous cycle. J Neurosci 1999; $19: 8122-33$

McDonald RJ. Multiple combinations of co-factors produce variants of age-related cognitive decline: A theory. Can J Exp Psycol 2002; 56:221-239.

McGahon BM, Clements MP, Lynch MA. The ability of aged rats to sustain longterm potentiation is restored when the aged-related decrease in membrane arachidonic acid concentration is reversed. Neuroscience 1997; 81:9-16.

Meaney MJ, Aitken DH, Bhatnagar S, Sapolsky RM. Postnatal handling attenuates certain neuroendocrine, anatomical, and cognitive dysfunction associated with aging in female rats. Neurobiol Aging 1991; 12: 31-38.

Merrill DA, Karim R, Darraq M, Chiba A, Tuszynski MH. Hippocampal cell genesis does not correlate with spatial learning ability in aged rats. J Comp Neurol 459:201-207.

Metzler J, Shepard RN. Transformational studies of the internal representations of three-dimensional objects. In: Solso RL, ed. Theories of cognitive psychology: The Loyola symposium. Hillsdale, NJ: Earlbaum, 1974; 147-201. 
Milner B. Amnesia following operation on temporal lobe. In: Whitty CWM, Zangwill OL, eds. Amnesia. London, UK: Butterworth Press, 1966; 109-133.

Minematsu K, Li L, Fischer M, Sotak CH, Davis MA, Fiandaca MS. Diffusionweighted magnetic resonance imaging: rapid and quantitative detection of focal brain ischemia. Neurology 1992; 42:235-240.

Mishkin M, Malamut B, Bachevalier J. Memories and habits: Two neural systems. In: Lynch G, McGaugh L, Weinberger NM, eds. Neurobiology of learning and memory. New York: Guilford, 1984; 65-77.

Mjaatvedt AE, Wong-Riley MTT. Effects of unilateral climbing fiber deafferentation on cytochrome-oxidase activity in the developing rat cerebellum. Develop Biol 1991; 145:219-230.

Moffat SD, Hampson E, Hatzipantelis M. Navigation in a "virtual" maze: sex differences and correlation with psychometric measures of spatial ability in humans. Evol Hum Behav 1998; 19:73-87.

Moffat SD, Resnick SM. Effects of age on virtual environment place navigation and allocentric cognitive mapping. Behav Neurosci 2002; 116:851-859.

Moffat SD, Zonderman AB, Resnick SM. Age differences in spatial memory in a virtual environment navigation task. Neurobiol Aging 2001; 22:787-796.

Moffett JR, Namboodiri MA, Cangro CB, Neale JH. Immunohistochemical localization of $\mathrm{N}$-acetylaspartate in rat brain. Neuroreport $1991 ; 2: 131-134$.

Morris RGM. Spatial localization does not require the presence of local cues. Learn Motiv 1981; 12:239-260.

Morris RGM. Modeling amnesia and the study of memory in animals. Trends Neurosci 1983; 6:479-483.

Morris RGM, Garrud P, Rawlins JNP, O'Keefe J. Place navigation impaired in rats with hippocampal damage. Nature 1982; 297:681-683.

Morris RGM, Anderson E, Lynch GS, Baudry M. Selective impairment of learning and blockade of long-term potentiation by an $\mathrm{N}$-methyl-D-aspartate receptor anatgonist, AP5. Nature 1986; 319: 774-776.

Moser E, Moser MB, Andersen P. Spatial learning impairment parallels the magnitude of dorsal hippocampal lesions, but is hardly present following ventral lesions. ] Neurosci 1993; 13:3916-3925.

Moser MB, Moser EI. Functional differences in the hippocampus. Hippocampus $1998 ; 8: 608-619$. 
Murray CA, Lynch MA. Evidence that increased hippocampal expression of cytokine interleukin-1 $\beta$ is a common trigger for age and stress-induced impairments in long-term potentiation. J Neurosci 1988a; 8:2974-2981.

Murray CA, Lynch MA. Dietary supplementation with vitamin E reverses the agerelated deficit in long-term potentiation in dentate gyrus. J Biol Chem 1988b; 273:12161-12168.

Nadel L, Moscovitch M. The hippocampal complex and long-term memory revisited. Trends Cog Sci 2001; 5:228-230.

Naressi A, Couturier C, Devos JM, Janssen M, Mangeat C, de Beer R, GraveronDemilly D. Java-based Graphical User Interface for the MRUI Quantitation Package. Magn Reson Material Phys Biol Med 2001; 12:141-152.

Nicholls DG. Release of glutamate, aspartate, and -aminobutyric acid from isolated nerve terminals. J Neurochem 1989; 52:331-41.

Nilsson $M$, Perfilieva $E$, Johansson $U$, Orwar $O$, Eriksson PS. Enriched environment increases neurogenesis in the adult rat dentate gyrus and improves spatial memory. J Neurobiol 1999; 39:569-578.

Norman AB, Bertram KJ, Thomas SR, Pratt RG, Samaratunga RC, Sanberg PR. Magnetic resonance imaging of rat brain following in vivo disruption of the cerebral vasculature. Brain Res Bull 1991; 26:593-597.

O'Keefe J, Conway DH. Hippocampal place units in freely moving rat-why they fire where they fire. Exp Brain Res 1978; 31:573-590.

O'Keefe J, Dostrovsky J. The hippocampus as a spatial map. Preliminary evidence from unit activity in the freely moving rat. Brain Res 1971; 34:171175.

O'Keefe J, Nadel L. The hippocampus as a cognitive map. Oxford, UK: Clarendon Press, 1978.

O'Reilly RC, Rudy JW. Conjunctive representations in learning and memory: Principles of cortical and hippocampal function. Psychol Rev 2001; 108, 311345.

Olson JE, Katz-Stein A, Reo NV, Jolesz FA. Evaluation of acute brain edema using quantitative magnetic resonance imaging: effects of pretreatment with dexamethasone. Magn Reson Med 1992; 24:64-74.

Olton DS, Samuelson RJ. Remembrance of places passed: Spatial memory in rats. J Exp Psychol: Anim Behav Process 1976; 2:97-116. 
Osteen WK, Spencer RL, Bare DJ, McEwen BS. Analysis of severe photoreceptor loss and Morris water-maze performance in aged rats. Behav Brain Res 1995; 68:151-158.

Packard MG, White NM. Dissociation of hippocampus and caudate nucleus memory systems by posttraining intracerebral injections of dopamine agonists. Behav Neurosci 1991; 105:295-306.

Paxinos G, Watson C. The rat brain in stereotaxic coordinates. New York: Academic Press, 1986.

Pavlakis SG, Lu D, Frank Y, Wiznia A, Eidelberg D, Barrnett T, Hyman RA. Brain lactate and $\mathrm{N}$-acetylaspartate in pediatric AIDS encepalopathy. Am J Neuroradiol $1998 ; 119: 383-385$.

Pelleymounter MA, Smith MY, \& Gallagher M. Spatial learning impairments in aged rats trained with a salient configuration of stimuli. Psychobiology 1987; $15: 248-254$.

Perlmutter M, Metzger R., Nezworski T, Miller K. Spatial and temporal memory in 20 and 60 year olds. 1 Gerontol 1981; 36:59-65.

Petersen RC, Jack CR, Smith GE, Warning SC, Ivnik RJ. MRI in the diagnosis of Mild Cognitive Impairment and Alzheimer's disease, J Int Neuropsychol Soc $1998 ; 4: 22$.

Petersen RC, Jack $C R$, Xu YC, Waring SC, O'Brien PC, Smith GE, Ivnik RJ, Tangalos EG, Boeve BF, Kokmen E. Memory and MRI-based hippocampal volumes in aging and AD. Neurology 2000; 54:581-587.

Pierce JM, Hall G. A model for Pavlovian learning - variations in the effectiveness of conditioned but not of unconditioned stimuli. Psych Rev 1980; 87:532-552.

Poe BH, Linville C, Riddle DR, Sonntag WE, Brunso-Bechtold JK. Effects of age and insulin-like growth factor-1 on neuron and synapse numbers in area CA3 of hippocampus. Neuroscience 2001; 107:231-238.

Prusky GT, West PWR, Douglas RM. Behavioral assessment of visual acuity in mice and rats. Vision Res 2000a; 40:2201-2209.

Prusky GT, West PWR, Douglas RM. Reduced visual acuity impairs place but not cued learning in the Morris water task. Beh Brain Res 2000b; 116:135-140.

Prusky GT, Harker KT, Douglas RM, Whishaw IQ. Variation in visual acuity within pigmented, and between pigmented and albino rat strains. Behavioral Brain Research 2002; 1 36:339-348. 
Prusky GT, Alam NM, Beekman S, Douglas RM. Rapid quantification of adult and developing mouse spatial vision using a virtual optomotor system. Investig Ophthal Vis Sci 2004; 45:4611-4616.

Rapp P, Rosenberg $R$, \& Gallagher $M$. An evaluation of spatial information processing. Behav Neurosci 1987; 101:3-12.

Rawlins JNP, Olton DS. The septohippocampal system and cognitive mapping. Behav Brain Res 1982; 5:331-358.

Raz N. Aging of the Brain and Its Impact on Cognitive Performance: Integration of Structural and Functional Findings. In: Craik FIM, Salthouse TA, eds. Handbook of Aging and Cognition. New Jersey: Erlbaum, 2000; 1-90.

Reed JM, Squire LR. Impaired transverse patterning in human amnesia is a special case of impaired memory for two-choice discrimination tasks. Behav Neurosci 1999; 113:3-9.

Rescorla RA, Wagner AR. A theory of Pavlovian conditioning: Variations in the effectiveness of reinforcement and nonreinforcement. In: Black AH, Prokasy WF, eds. Classical conditioning II: Current research and theory. New York: AppletonCentury-Crofts, 1972; 64-99.

Richmond MA, Yee BK, Pouzet B, Veenman L, Rawlins JNP, Felden J, Bannerman DM. Dissociating context and space within the hippocampus: Effects of complete dorsal, and entral excitotoxic hippocampal lesions on conditioned freezing and spatial learning. Behav Neurosci 1999; 113:1189-1203.

Rickard TC, Grafman J. Losing their configural mind: Amnesic patients fail on transverse patterning. J Cogn Neurosci 1998; 10:509-524.

Rondi-Reig L, Libbey M, Eichenbaum H, Tonegawa S. CA1-specific N-methyl-Daspartate receptor knockout mice are deficient in solving non-spatial transverse patterning task. Proc Natl Acad Sci USA 2001; 98:3543-3548.

Ross RT, Orr WB, Holland PC, Berger TW. Hippocampectomy disrupts acquisition and retention of learned conditional responding. Behav Neurosci 1984; 98:211225.

Rosene DL, Van Hoesen GW. Hippocampal efferents reach widespread areas of cerebral cortex and amygdala in rhesus-monkey. Science 1977; 198:315-317.

Rosenzweig ES, Barnes CA. Impact of aging on hippocampal function : plasticity, network dynamics, and cognition. Prog Neurobiol 2003; 69:143-179.

Rudy JW, Sutherland RJ. Configural association theory and the hippocampal formation: an appraisal and reconfiguration. Hippocampus 1995; 5:375-389. 
Rudy JW, Keith JR, Georgen K. The effect of age in childrens learning of problems that require a configural association solution. Dev Psychobiol 1993; 26:171-184.

Rudy JW, O'Reilly RC. Conjunctive representations, the hippocampus, and contextual fear conditioning. Cog Affect Behav Neurosci 2001; 1:66-82.

Rudy JW, Huff N, Matus-Amat P. Understanding contextual fear conditioning: Insights from a two process model. Neurosci Biobehav Rev 2004;28, 675-686.

Ryan L, Nadel L, Keil K, Putnam K, Schnyer D, Trouard T, Moscovitch M. The hippocampal complex and retrieval of recent and very remote autobiographical memories: Evidence from functional magnetic resonance imaging in neurologically intact people. Hippocampus 2001; 11:707-714.

Sara SJ. Memory deficits in rats with hippocampal or cortical lesions: retrograde effects. Behav Neural Biol 1981; 32:504-509.

Saunders JK, Smith IC, MacTavish JC, Rydzy M, Peeling J, Sutherland E, Lesiuk $H$, Sutherland GR. Forebrain ischemia studied using magnetic resonance imaging and spectroscopy. NMR Biomed 1989; 2:312-316.

Schuff N, Marmar CR, Weiss DS, Neylan TC, Schoenfeld F, Fein G, Weiner MW. Reduced hippocampal volume and $\mathrm{N}$-acetylaspartate in post-traumatic stress disorder. Ann N Y Acad Sci 1997a; 821:516-520.

Schuff N, Amend D, Ezekiel F, Steinman SK, Tanabe J, Norman D, Jagust W, Kramer JH, Mastrianni JA, Fein G, Weiner MW. Changes of hippocampal Nacetylaspartate and volume in Alzheimer's disease. A proton MR spectroscopic imaging and MR study. Neurology 1997b; 49:1513-1521.

Schuff N, Amend DL, Knowlton R, Norman D, Fein G, Weiner MW. Age-related metabolite changes and volume loss in the hippocampus by magnetic resonance spectroscopy and imaging. Neurobiol Aging 1999; 20:279-285.

Scoville WB, Milner B. Loss of recent memory after bilateral hippocampal lesions. J Neurol Neurosurg Psychiatry 1957; 20:11- 21.

Seilhean D, Duyckaerts C, Vazeux R, Bolgert F, Brunet P, Katlama C, Gentilini M, Hauw JJ. HIV-1-associated cognitive motor complex - absence of neuronal loss in the cerebral neocortex. Neurology 1993; 43:1492-1499.

Shingo T, Sorokan ST, Shimazaki T, Weiss S. Erythropoietin regulates the in vitro and in vivo production of neuronal progenitors by mammalian forebrain neural stem cells ] Neurosci 2001; 21:9733-9743. 
Shore DI, Stanford L, MacInnes WJ, Klein RM, Brown RE. Of mice and men: Virtual Hebb-Williams mazes permit comparison of spatial learning across species. Cognitive, Affective, and Behav Neurosci 2001; 1:83-89.

Snyder JS, Kee N, Wojtowicz JM. Effects of adult neurogenesis on synaptic plasticity in the rat dentate gyrus. J Neurophys 2001; 85:2423-2431.

Sokoloff L. Energetics of functional activation in neural tissues. Neurochem Res 1999; 24:321-29.

Spence $\mathrm{KW}$. The nature of the response in discrimination learning. Psychol Rev $1952 ; 59: 89-93$

Squire LR. Memory and the hippocampus: A synthesis from findings with rats, monkeys, and humans. Psychol Rev 1992; 99:195-231.

Stanfield $\mathrm{BB}$, Trice JE. Evidence that granule cells generated in the dentate gyrus of adult rats extend axonal projections. Exp Brain Res 1988; 72:399-406.

Sterio DC. The unbiased estimation of number and sizes of arbitrary particles using the disector. J Microsci 1984; 134:127-136.

Sullivan EV, Marsh L, Mathalon DH, Lim KO, Pfefferbaum A. Age-related decline in MRI volumes of temporal lobe gray matter but not hippocampus. Neurobiol Aging 1995; 16:591-606.

Sutherland RJ, Chew GL, Baker JC, Linggard RC. Some limitations on the use of distal cues in place navigation by rats. Psychobiology 1987; 15:48-57.

Sutherland RJ, Kolb B, Whishaw IQ. Spatial mapping: definitive disruption by hippocampal or medial frontal cortical damage in the rat. Neurosci Lett 1982a; $31: 271-276$

Sutherland RJ, Rudy JW. Configural association theory: the role of the hippocampal formation in learning, memory, and amnesia. Psychobiology 1989; 17:129-144.

Sutherland RJ, Weisend MP, Mumby D, Astur RS, Hanlon FM, Koerner A, Thomas MJ, Wu Y, Moses SN, Cole C, Hamilton DA, Hoesing JM. Retrograde amnesia after hippocampal damage: Recent vs, remote memories in two tasks. Hippocampus $2001 ; 11: 27-42$.

Sutherland RJ, Whishaw IQ, Regehr JC. Cholinergic receptor blockade impairs spatial localization by use of distal cues in the rat. J Comp Physiol Psychol 1982b; 96:563-573. 
Tatebayashi Y, Lee MH, Li L, Iqbal K, Grundke-Iqbal I. The dentate gyrus neurogenesis: a therapeutic target for Alzheimer's disease. Acta Neuropathol $2003 ; 105: 225-232$.

Tattersfield AS, Croon RJ, Liu YW, Kells AP, Faull RLM, Connor B. Neurogenesis in the striatum of the quinolinic acid lesion model of Huntington's disease. Neuroscience 2004; 127:319-332.

Tielen AM, Mollevanger WJ, Lopes de Silva FH, Hollander CF. Neuronal plasticity in hippocampal slices of extremely old rats. In: Gispen WH, Traber J (Eds.). Aging of the Brain. Elsevier, Amsterdam 1983; 73-84.

Tulving E. Episodic and semantic memory - Where should we go from here. Behav Brain Sci 1986; 9:573-577.

Tyler TJ, DiScenna $P$. The hippocampus memory indexing theory. Behav Neurosci 1986; 100:147-152.

Urenjak J, Williams SR, Gadian DG, Noble M. Proton nuclear magnetic resonance spectroscopy unambiguously identifies different neural cell types. J Neurosci $1993 ; 13: 981-989$.

Van der Staay FJ. Assessment of age-associated cognitive deficits in rats: a tricky business.

Neurosci Biobeh Rev 2002; 26:753-759.

Van der Staay FJ, de Jonge $M$. Effects of age on water escape behavior and on repeated acquisition in rats. Behav Neural Biol 1993; 60:33-41.

Van Elzakker M, O'Reilly RC, Rudy JW. Transitivity, flexibility, conjunctive representations, and the hippocampus. I. An empirical analysis. Hippocampus 2003; 13: 334-340.

van Praag $\mathrm{H}$, Kempermann G, Gage $\mathrm{FH}$. Running increases cell proliferation and neurogenesis in the adult mouse dentate gyrus. Nat Neurosci $1999 ; 2: 266-270$.

van Praag H, Schnider AF, Christie BR, Toni N, Palmer TD, Gage FH. Functional neurogenesis in the hippocampus. Nature 2002; 415:1030-1034.

Vandenberg S, Kuse AR. Mental rotations: A group test of three-dimensional spatial visualization. Percept Mot Skills 1978; 47:599-604.

Vijg J. Somatic mutations and aging: a re-evaluation. Mutat Res 2000; 447:11735.

Wagner JP, Black IB, DiCicco-Bloom E. Stimulation of neonatal and adult brain neurogenesis by subcutaneous injection of basic fibroblast growth factor. ] Neurosci 1999; 19:6006-6016. 
Walker JA, Olton DS. Spatial memory deficit following fimbria-fornix lesions independent of time for stimulus processing. Physiol Bhav 1979; 23:11-15.

Wang R, Dineley KT, Sweatt JD, Zheng $H$. Presenilin 1 familial Alzheimer's disease mutation leads to defective associative learning and impaired adult neurogenesis. Neuroscience 2004; 126; 2:305-312.

Weisend M, Astur R, Sutherland RJ. The specificity and temporal characteristics of retrograde amnesia after hippocampal lesions. Soc Neurosci Abstract 1996; 22:1118.

West MJ. Regionally specific loss of neurons in the aging hippocampus. Neurobiol Aging 1993; 14:287-293.

Wickelgren WA. Chunking and consolidation: a theoretical synthesis of semantic networks, configuring in conditioning, S-R versus cognitive learning, normal forgetting, the amnesic syndrome, and the hippocampal arousal system. Psychol Rev 1979; 86:44-60.

Winocur $G$. Anterograde and retrograde amnesia in rats with dorsal hippocampal or dorsomedial thalamic lesions. Behav Brain Res 1990; 38:145-154.

Winocur G, McDonald RM, Moscovitch M. Anterograde and retrograde amnesia in rats with large hippocampal lesions. Hippocampus 2001; 11:18-26.

Wolf OT, Dyakin V, Patel A, Vadasz C, de Leon MJ, McEwen BS, Bulloch K. Volumetric structural magnetic resonance imaging (MRI) of the rat hippocampus following kainic acid (KA) treatment. Brain Research 2002; 934:87-96.

Wong-Riley MTT. Cytochrome oxidase - An endogenous metabolic marker for neuronal activity. Trend Neurosci 1989; 12:94-101.

Wyss JM, Chambless BD, Kadish I, van Groen T. Age-related decline in water task learning and memory in rats: strain differences. Neurobiol Aging 2000; $12: 671-81$

Zaharia MD, Kulczycki J, Shanks N, Meaney MJ, Anisman H. The effects of early postnatal stimulation on water-maze acquisition in adult mice: genetic and maternal factor. Psychopharmacology 1996; 128:227-39. 


\begin{tabular}{|c|c|c|c|c|}
\hline \multirow[t]{2}{*}{ Group } & \multirow[t]{2}{*}{ Condition } & \multicolumn{3}{|c|}{ Training Phase } \\
\hline & & 1 & 2 & 3 \\
\hline \multirow[t]{5}{*}{ ED } & Pre-lesion & $A+B$ & $\begin{array}{l}A+B- \\
C+D-\end{array}$ & $\begin{array}{l}A+B- \\
C+D- \\
E+F-\end{array}$ \\
\hline & Post-lesion (Test) & $\begin{array}{l}A+B- \\
C+D- \\
E+F-\end{array}$ & & \\
\hline & Post-lesion (Retrain) & $A+B$ & $\begin{array}{l}A+B- \\
C+D-\end{array}$ & $\begin{array}{l}A+B- \\
C+D- \\
E+F-\end{array}$ \\
\hline & $\begin{array}{l}\text { Post-lesion (Retest) } \\
\text { (10-day delay Test) }\end{array}$ & $\begin{array}{l}A+B- \\
C+D- \\
E+F=\end{array}$ & & \\
\hline & $\begin{array}{l}\text { Post-lesion } \\
\text { (New Problem) }\end{array}$ & $\mathrm{G}+\mathrm{H}-$ & $\underset{\mathrm{G}+\mathrm{H}-}{\mathrm{I}+\mathrm{J}-}$ & $\begin{array}{l}G+H- \\
I+J- \\
K+L-\end{array}$ \\
\hline \multirow[t]{3}{*}{$\overline{T P}$} & Pre-lesion & $X+Y-$ & $\begin{array}{l}X+Y- \\
Y+Z-\end{array}$ & $\begin{array}{l}X+Y- \\
Y+Z- \\
Z+X-\end{array}$ \\
\hline & Post-lesion (Test) & $\begin{array}{l}X+Y- \\
Y+Z- \\
Z-X+\end{array}$ & & \\
\hline & Post-lesion (Retrain) & $X+Y-$ & $\begin{array}{l}X+Y- \\
Y+Z\end{array}$ & $\begin{array}{l}X+Y- \\
Y+Z- \\
Z+X-\end{array}$ \\
\hline
\end{tabular}

Table 1. Discrimination training procedures are shown for each group (ED = elemental discriminations; TP = transverse patterning), and divided by the phases they were trained in. 
A.

\begin{tabular}{|c|c|c|c|c|c|c|}
\hline & VOLUME & NAA & $\begin{array}{l}\text { NEURON } \\
\text { DENSITY }\end{array}$ & $\begin{array}{l}\text { SYNAPTIC } \\
\text { DENSITY }\end{array}$ & $\begin{array}{l}\text { MITOCHONDRIAL } \\
\text { DENSITY }\end{array}$ & NEUROGENESIS \\
\hline MWT & $\begin{array}{l}r=-.378 \\
p=.047\end{array}$ & $Y$ & $Y$ & $Y$ & $\mathbf{X}$ & $\begin{array}{l}r=-.543 \\
p=.003\end{array}$ \\
\hline TPDT & $\begin{array}{l}r=.488 \\
p=.008\end{array}$ & $\mathbf{Y}$ & Y & $\mathbf{X}$ & $\mathbf{X}$ & $\begin{array}{l}r=.481 \\
p=.011\end{array}$ \\
\hline
\end{tabular}

B.

\begin{tabular}{|c|c|c|c|c|c|c|}
\hline & VOLUME & $\overline{\text { NAA }}$ & $\begin{array}{l}\text { NEURON } \\
\text { DENSITY }\end{array}$ & $\begin{array}{l}\text { SYNAPTIC } \\
\text { DENSITY }\end{array}$ & $\begin{array}{l}\text { MITOCHONDRIAL } \\
\text { DENSITY }\end{array}$ & NEUROGENESIS \\
\hline MWT & $\underset{(p=.174)}{X}$ & $\mathbf{X}$ & $\mathbf{X}$ & $X$ & $y$ & $\begin{array}{c}r=-.543 \\
p=.003\end{array}$ \\
\hline TPDT & $\underset{(p=.166)}{\mathbf{X}}$ & $Y$ & $\mathbf{X}$ & $\mathbf{X}$ & $\mathbf{X}$ & $\begin{array}{l}r=.481 \\
p=.011\end{array}$ \\
\hline
\end{tabular}

Table 2. (A) Correlations between behavioral measures (MWT ad TPDT) and hippocampal volume, neuronal density, synaptic density, mitochondrial density, and neurogenesis. Both, reduction in volume and neurogenesis predict learning and memory deficits on hippocampus-dependent tasks. (B) Partial correlations, controlling for neurogenesis (DCX-labeled) cells, suggest that the age-related reduction in neurogenesis primarily accounts for hippocampus-dependent tasks learning and memory deficits and mediates the relationship with hippocampal volume. 


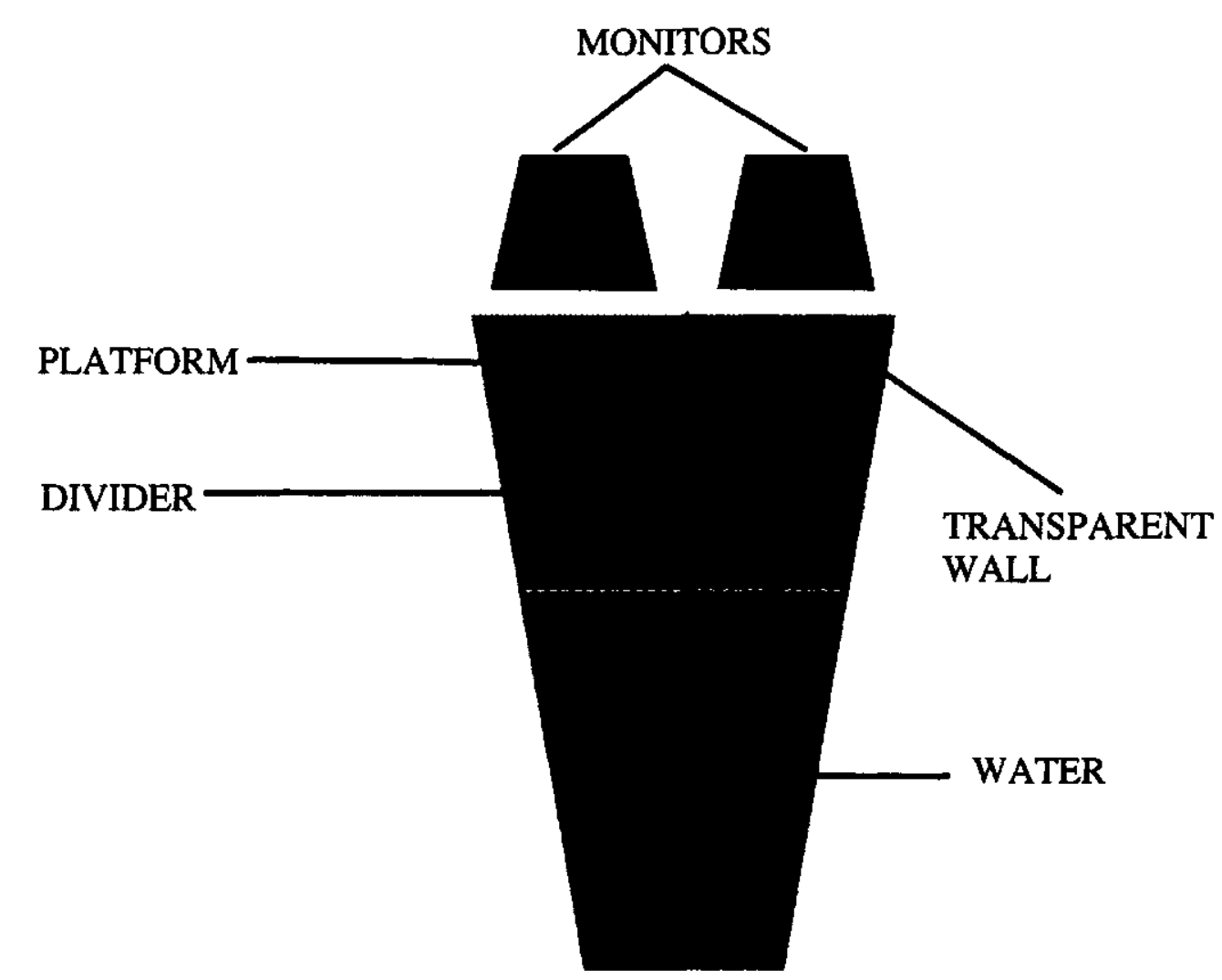

Figure 1. The Visual Water Task. Top view showing major components, including a trapezoidal-shaped pool, midline divider, platform, and computer monitors that display either reinforced $(+)$ or non-reinforced $(-)$ stimuli into the pool through a transparent wall. 


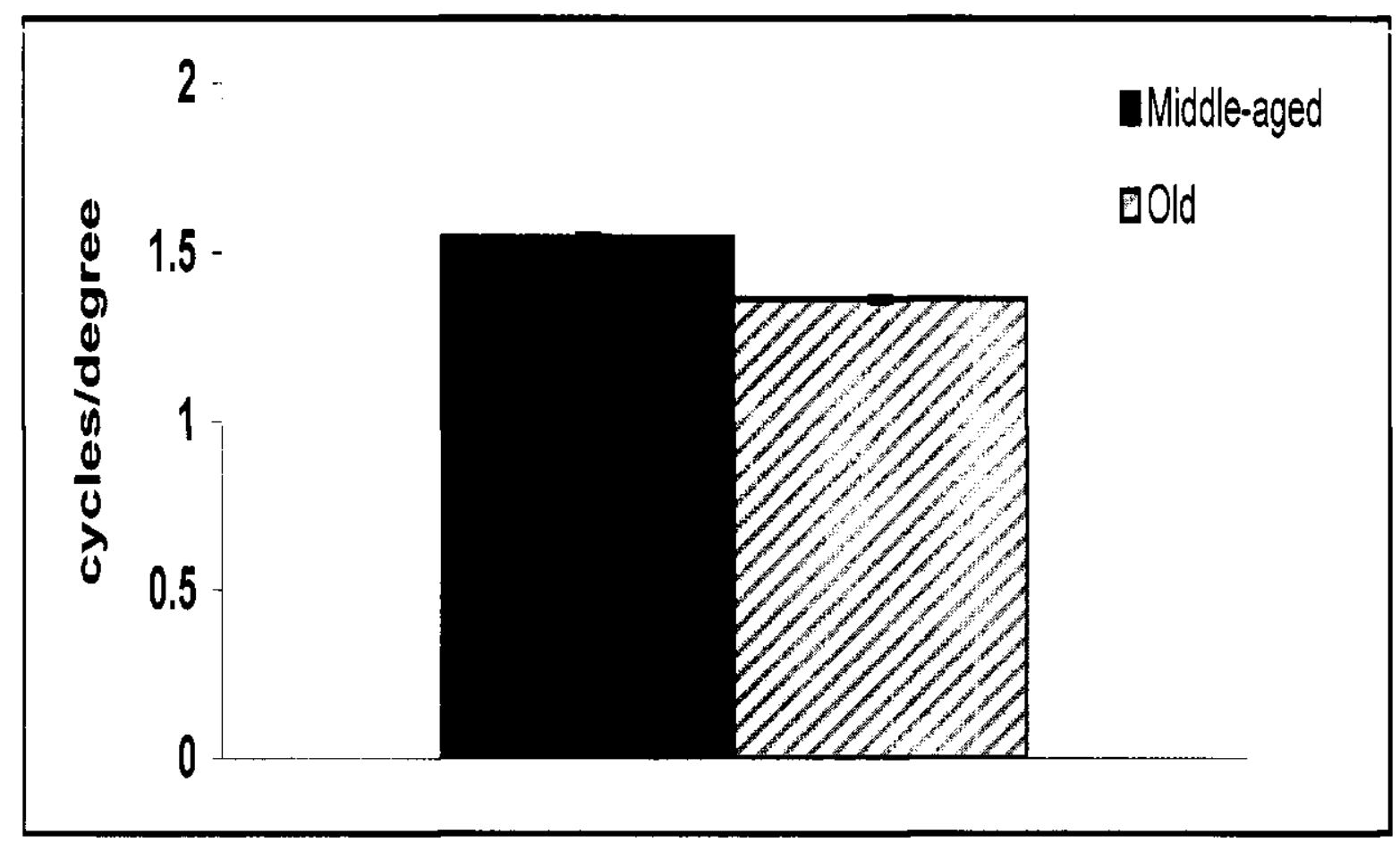

Figure 2. Acuity of middle-aged ( 14 months old) and old ( 26 months old) FBNF1 rats. The grating threshold acuity of Middle-aged FBNF1 rats clustered around $1.54 \mathrm{c} / \mathrm{d}$, which was significantly higher than that of the aged rats $(1.36$ $\mathrm{c} / \mathrm{d})$. 

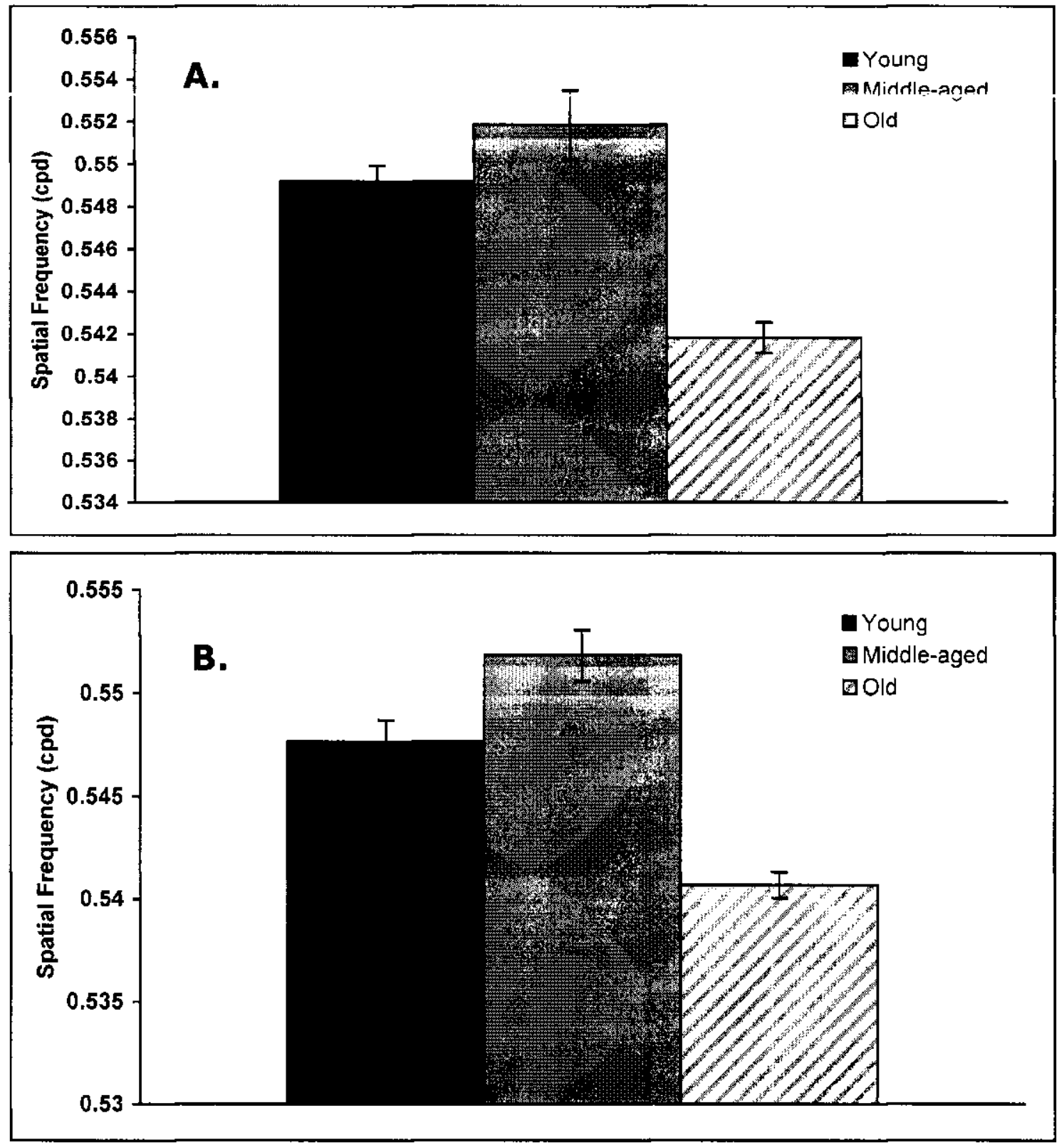

Figure 3 A \& B. The grating acuity as measured by the OptoMotor test. Old ( 26 months) FBNF1 rats had a significantly lower acuity compared to their young (4 moths) and middle-aged (14 months) counterparts when measured in both clockwise (A) and counterclockwise (B) directions. 
A.

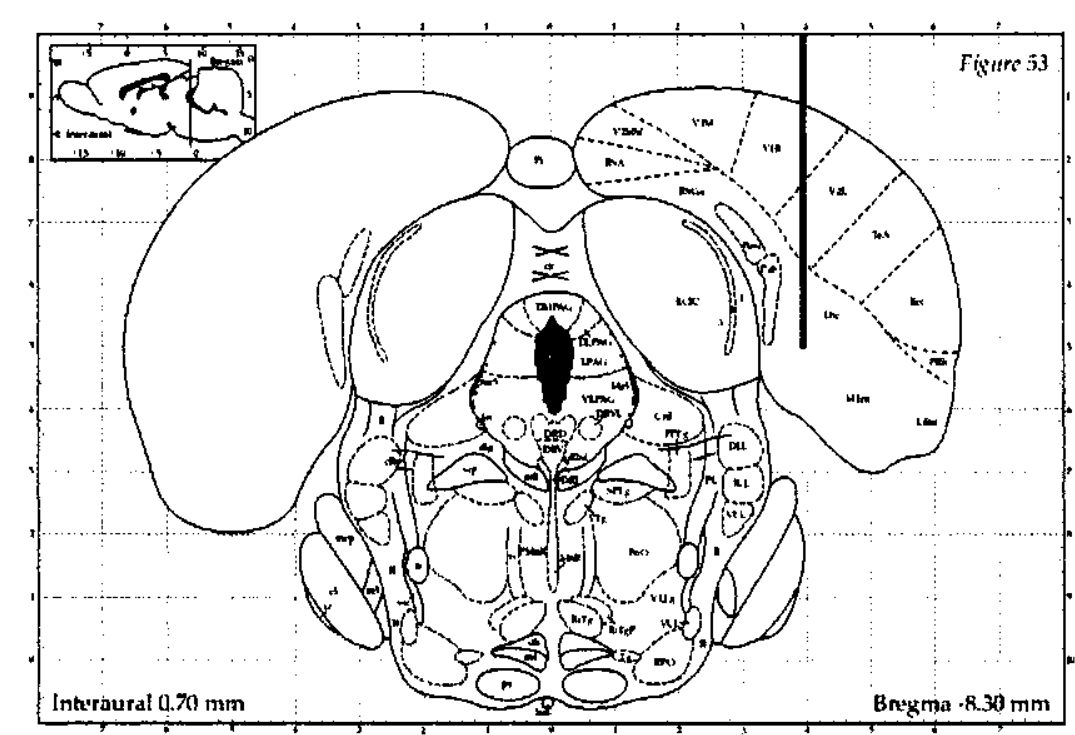

B.

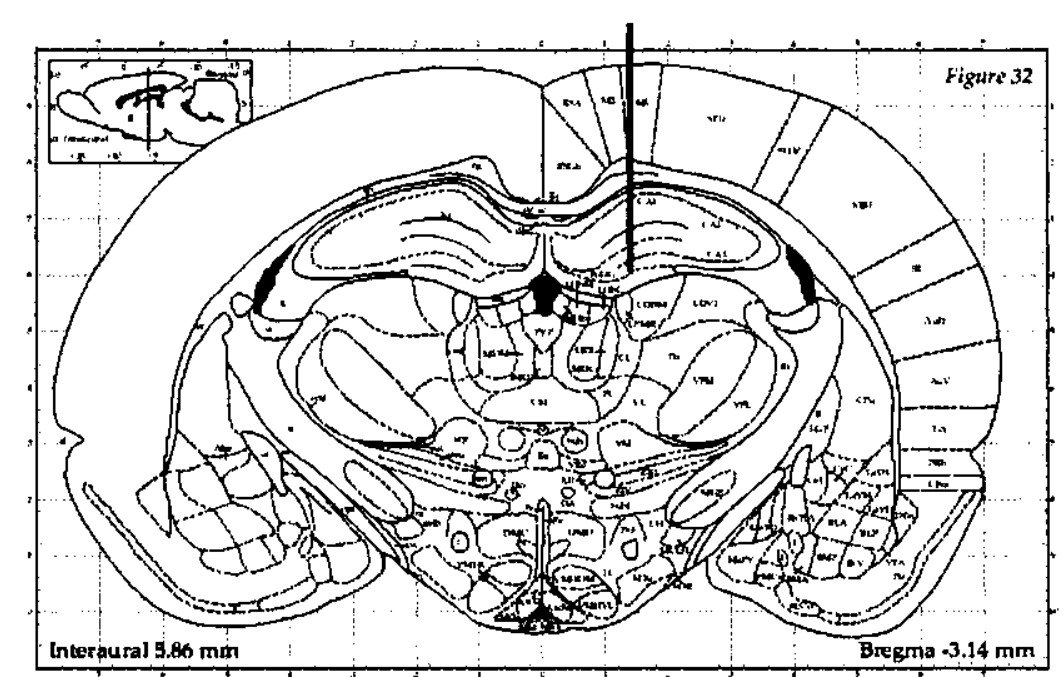

Figure 4. Electrophysiological recordings. Rats were implanted unilaterally with a stimulating electrode in the perforant path (A) and a recording (B) electrode in the dentate gyrus. 

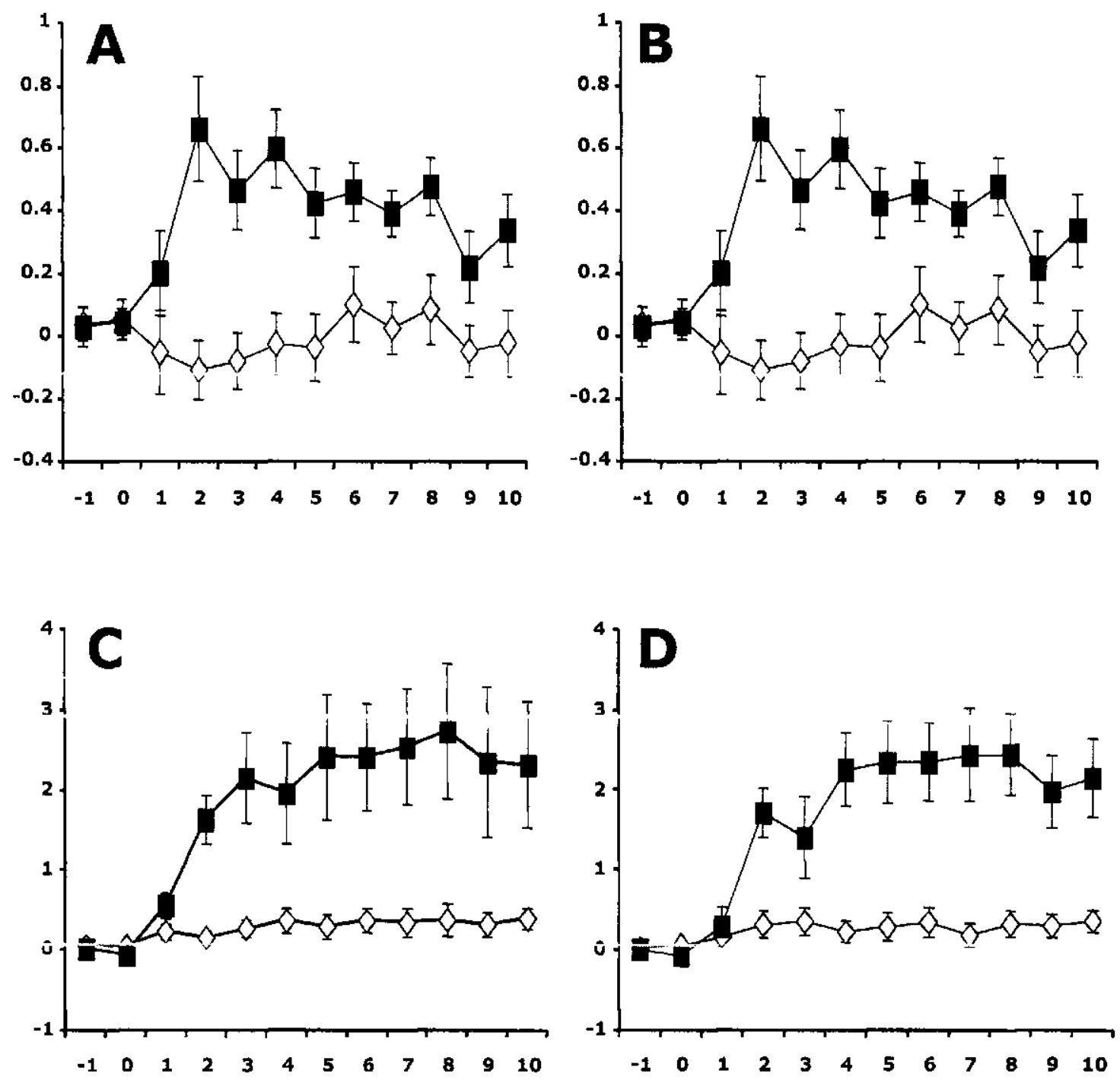

Figure 5. Effects of $10 \mathrm{HFS}$ sessions on the evoked potentials of middle-aged (white diamonds) and old (black squares) rats as measured 1 hour ( $A$ and $C$ ) and 24 hours ( $B$ and D) following stimulation. HFS resulted in a long-lasting increase in the population spike amplitude and the EPSP slope, an effect that could be observed both 1 and 24 hours following stimulation. However, middleaged rats showed a more pronounced increase in both the population spike amplitude (A \& B) and the EPSP slope (C\& D) compared to the old rats. ( $p<$ .05 for all comparisons). 


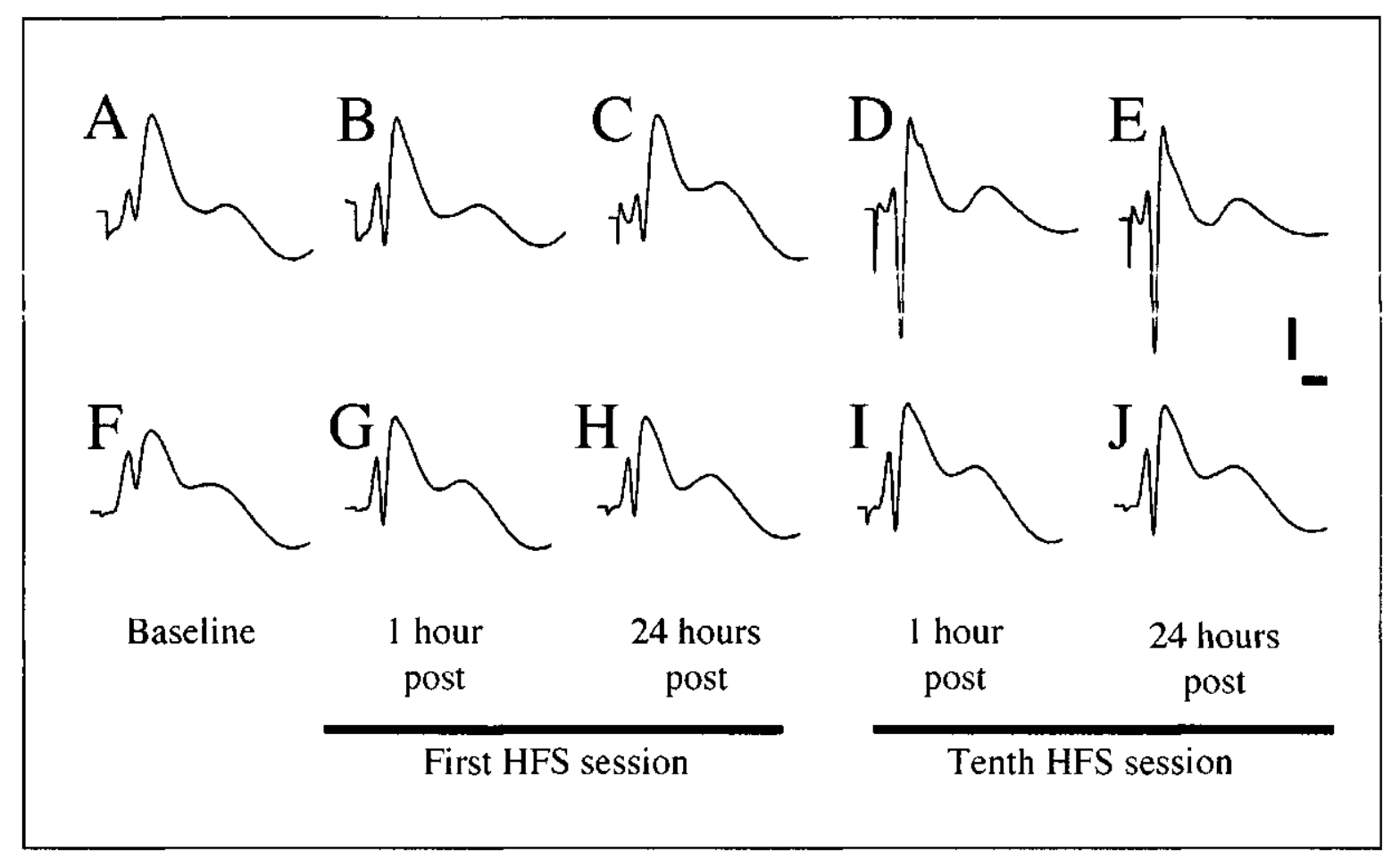

Figure 6. Effects of HFS on hippocampal field potentials in a representative middle-aged (top; A, B, C, D \& E) and old (bottom; F, G, H, I \& J) rat. The effects of the $1^{\text {st }}$ and $10^{\text {th }}$ session of HFS on field potentials of middle-aged and old rats 1 hour and 24 hours post stimulation are shown. In both groups, HFS induced a significant increase in population spike amplitude which was observable both 1 hour and 24 hours following the first HFS session. Relative to the old rats, the magnitude of change was enhanced in the middle-aged animals across the HFS sessions. (Calibration: vertical $=2 \mathrm{mV}$, horizontal $=2 \mathrm{~ms}$.) 
A.
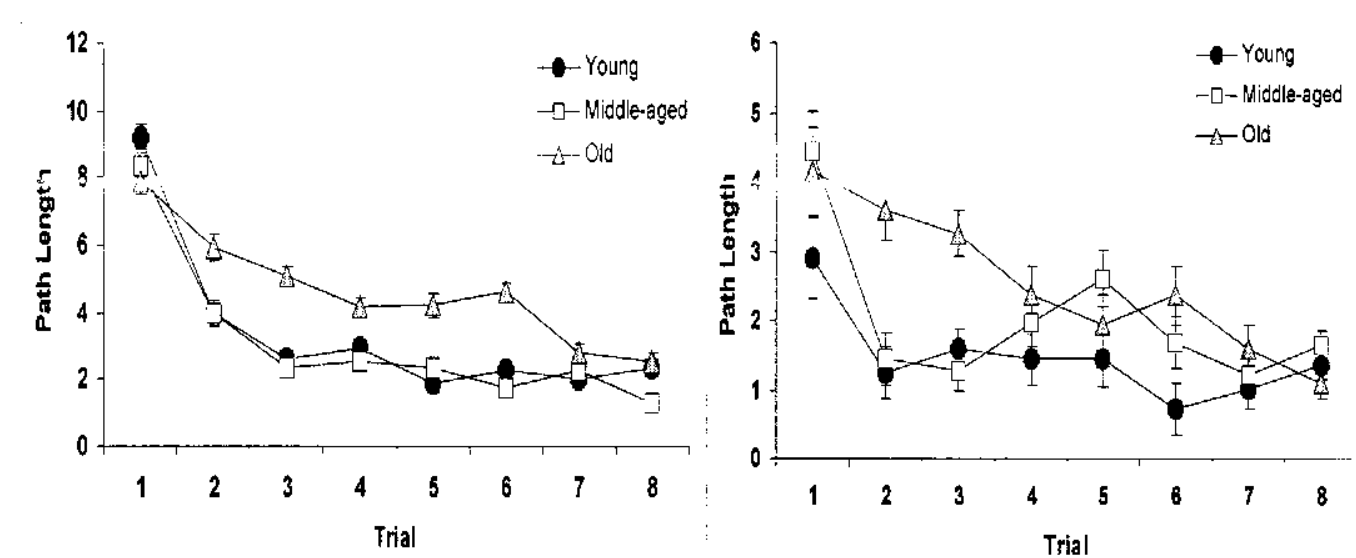

B.
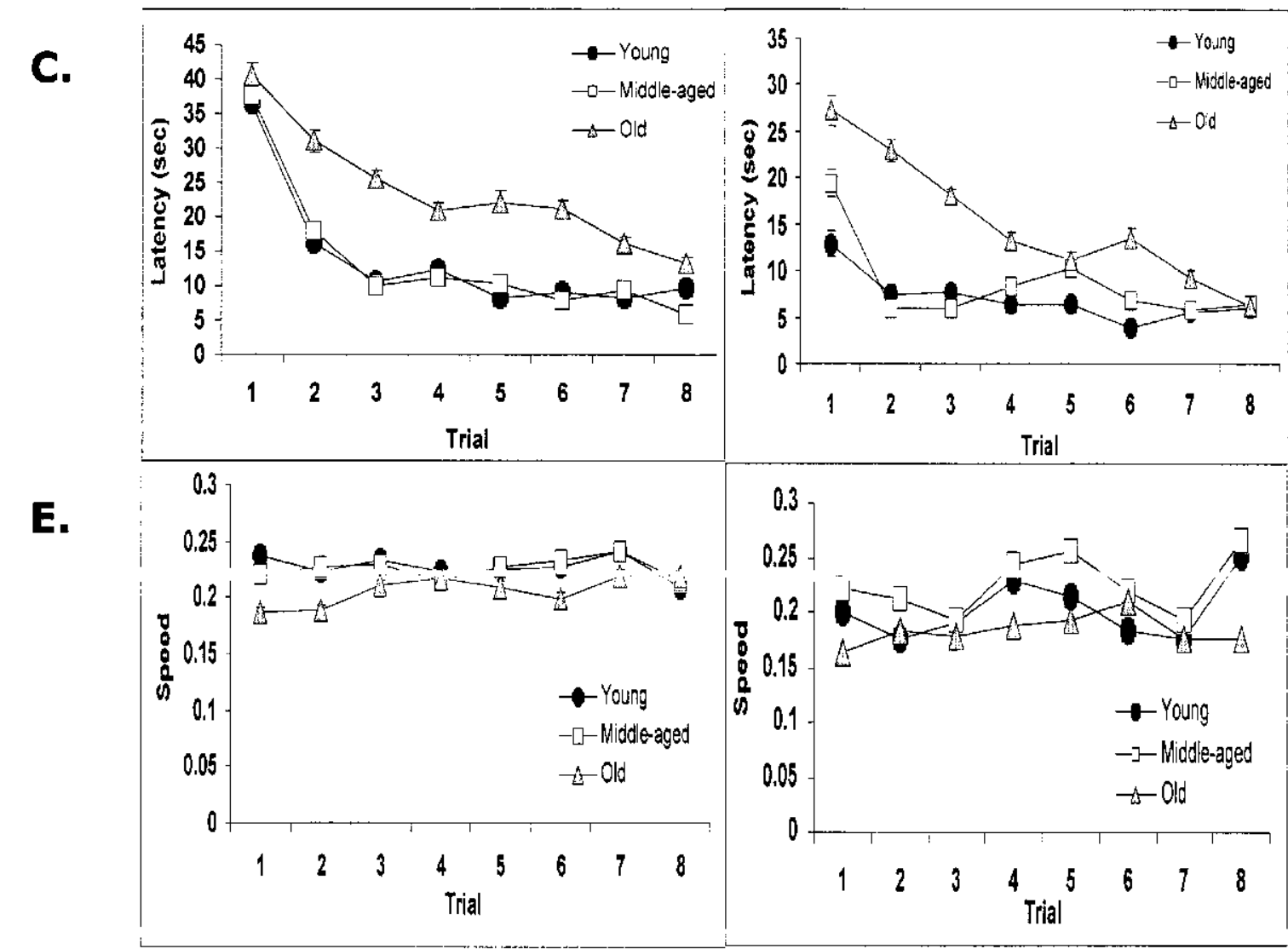

D.

F.

Figure 7. Performance on the hidden platform trials of the Morris water task. Performance was averaged across days when the platform was in a new location $(A, C$, and $E$ ) and days when the platform remained in the same location ( $B, D$, and $F$ ). Group (age) differences in latency (top) and path length (middle) to reach the platform, and in the speed of swimming (bottom) is presented. No clear pattern of deficits emerged in the acquisition of spatial information and the speed of swimming was comparable between groups. 


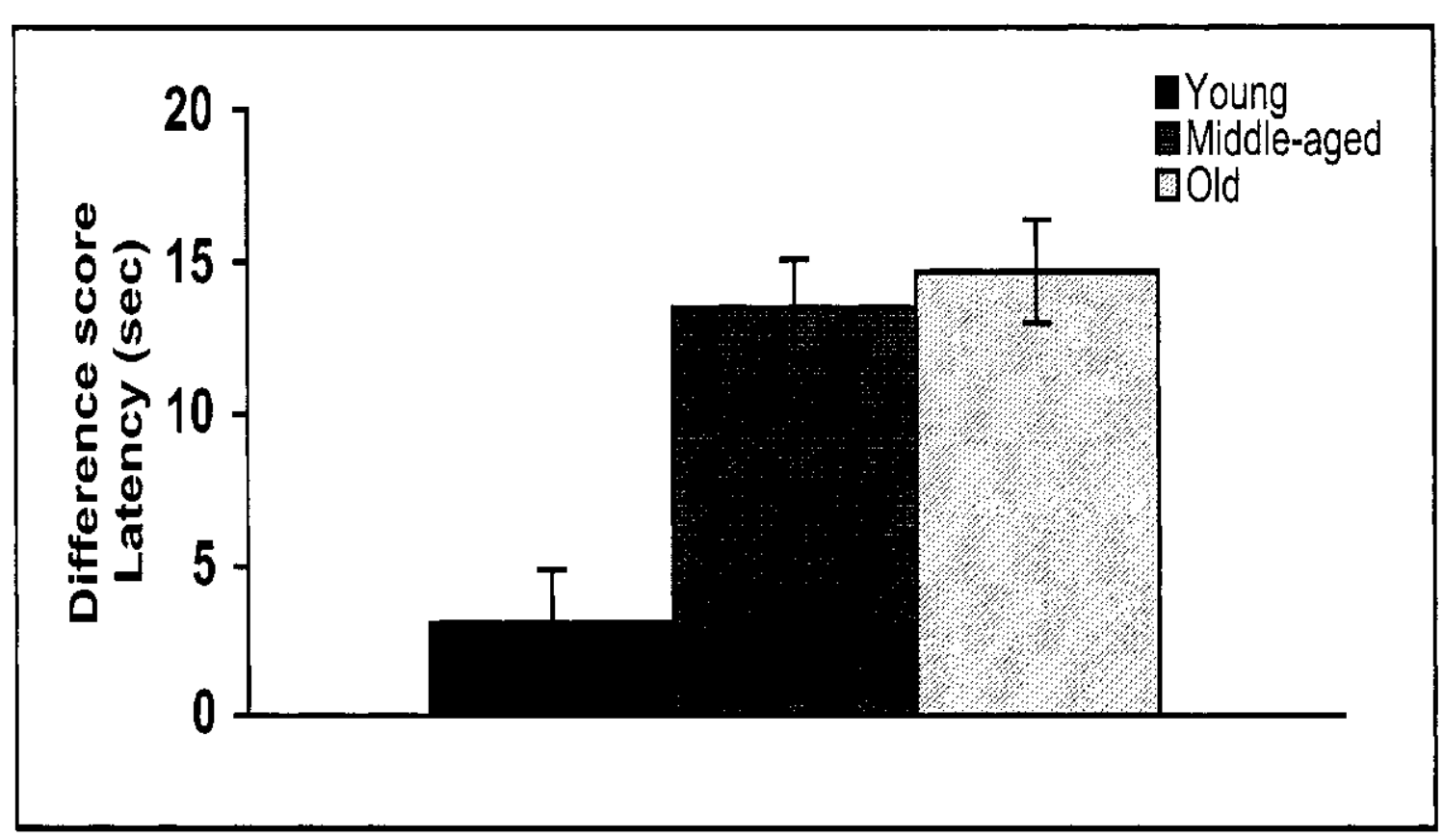

Figure 8. Retention scores representing the memory for the platform location experienced 24 hours earlier. Both middle-aged and old rats had trouble retaining the information related to the platform location learned on the previous day compared to the young rats. 


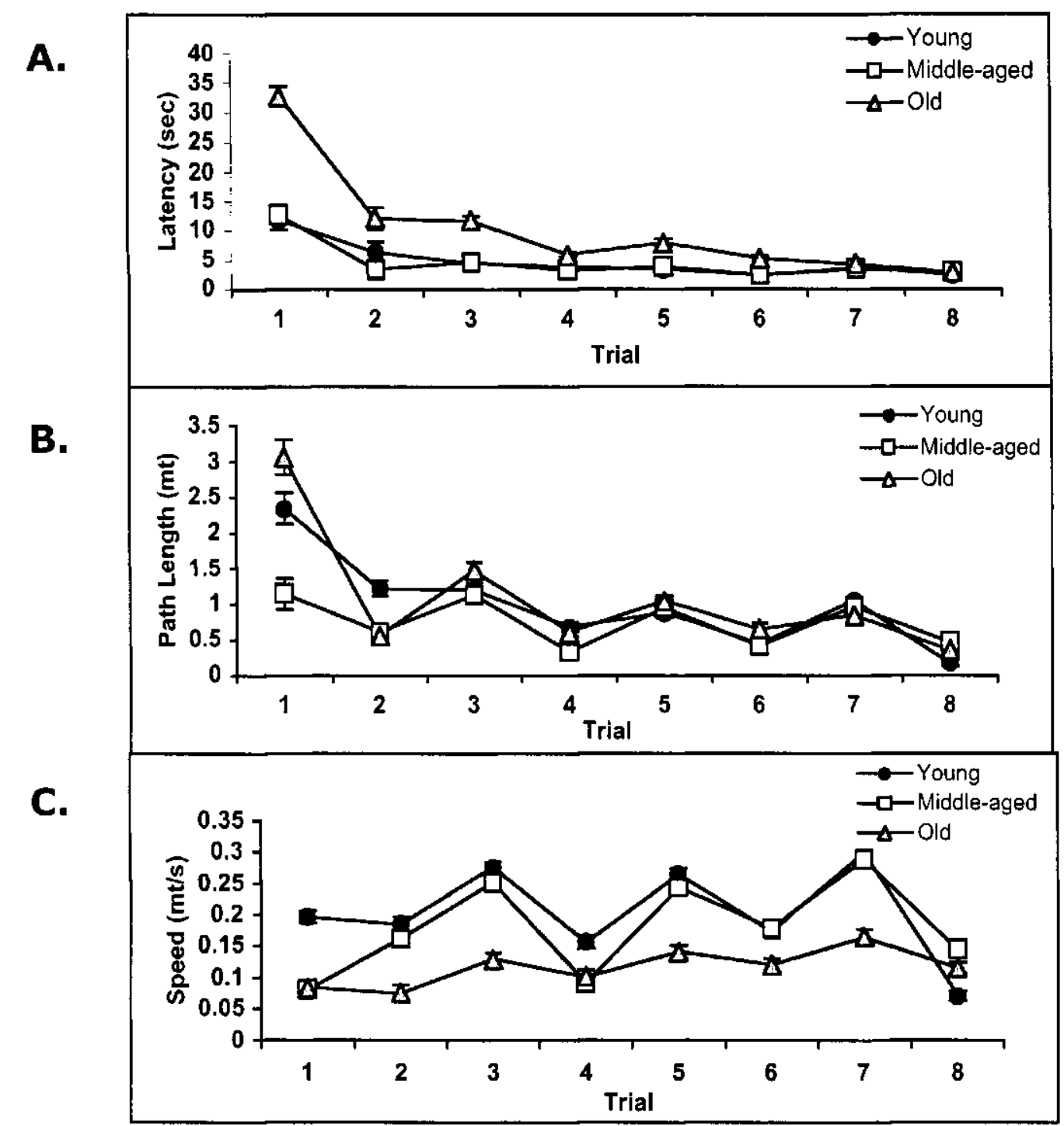

Figure 9. Performance on the visible platform trials of the Morris water task. Group (age) differences in latency (A) and path length (B) to reach the platform, and in the speed of swimming (C) across trials. Old rats can reach the same latencies and path lengths traveled to reach the visible platform as the young and middle-aged rats, which is especially evident on trials 4-8 as the rats become more familiar with the task. The speed of swimming is more variable, but they are capable of reaching the same speed of swimming as their younger counterparts. 


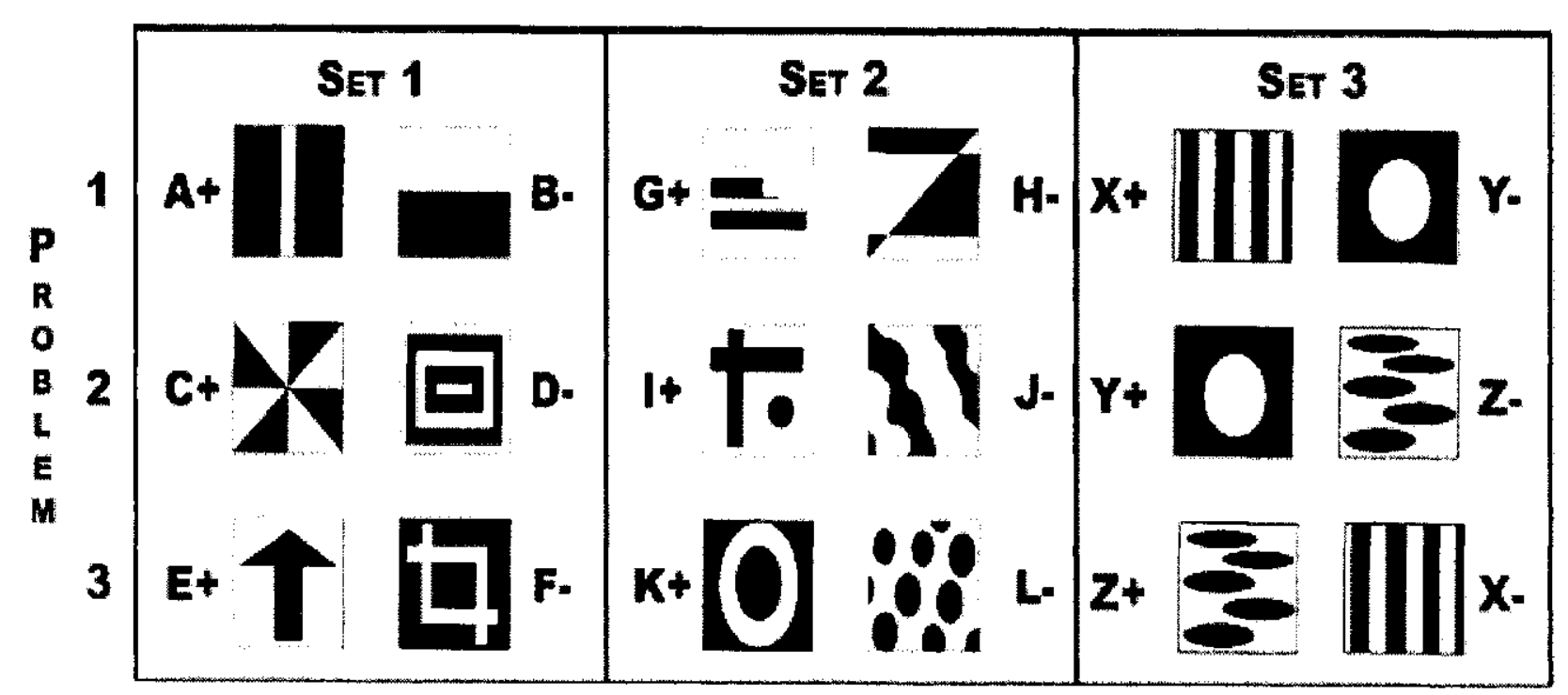

Figure 10. The discriminanda comprising elemental and transverse patterning problems. Elemental problems are presented in Sets 1 and 2 respectively, and the transverse patterning problem is presented in Set 3. 
A.

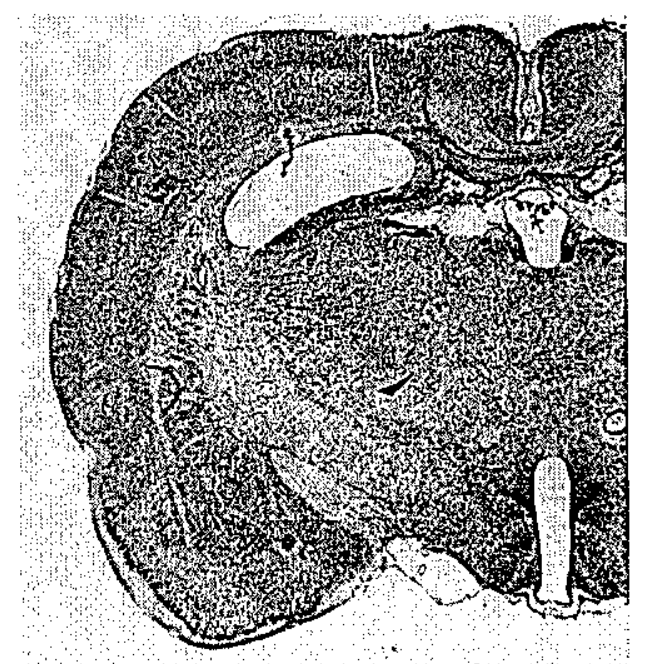

B.

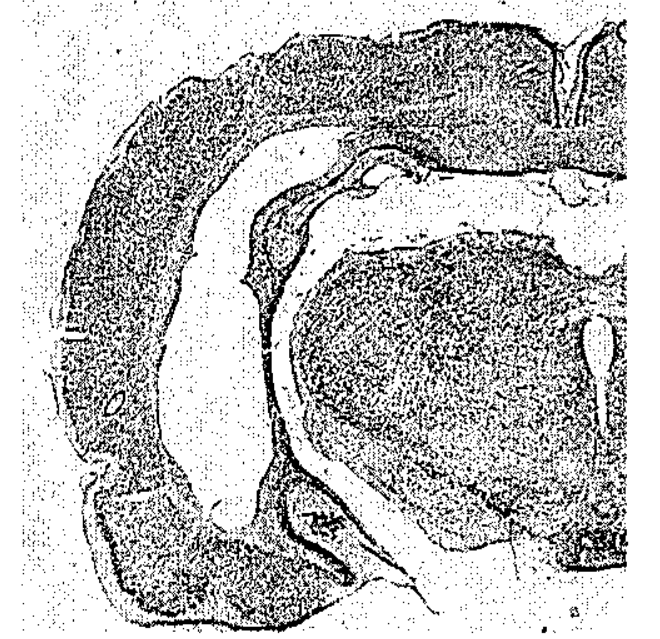

Figure 11. A photomicrograph of representative NMDA damage through dorsal (A.) and ventral (B.) hippocampal formation in coronal sections. 


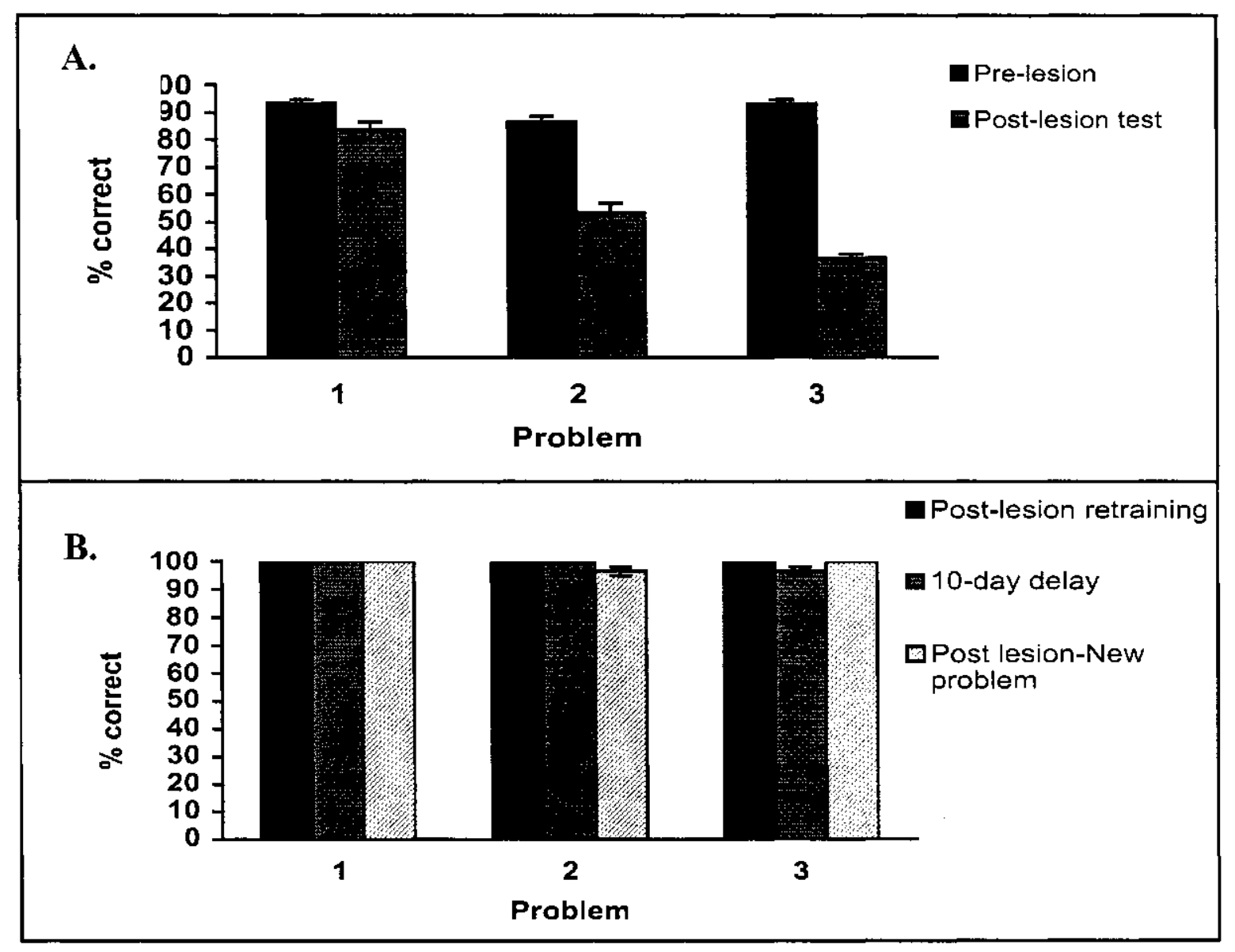

Figure 12. Performance on the elemental discrimination problems before and after the damage to the hippocampus. Rats with damage to the hippocampal formation exhibit retrograde amnesia for elemental discriminations learned prior to surgery (A). Rats with damage to the hippocampal formation do not exhibit anterograde amnesia for simple discriminations - they can re-learn the set of elemental problems they were trained on before the damage, learn a new set of elemental problems, and retain it over a 10-day delay without the hippocampus (B). $\left(p^{\prime} s<0.05\right)$. 


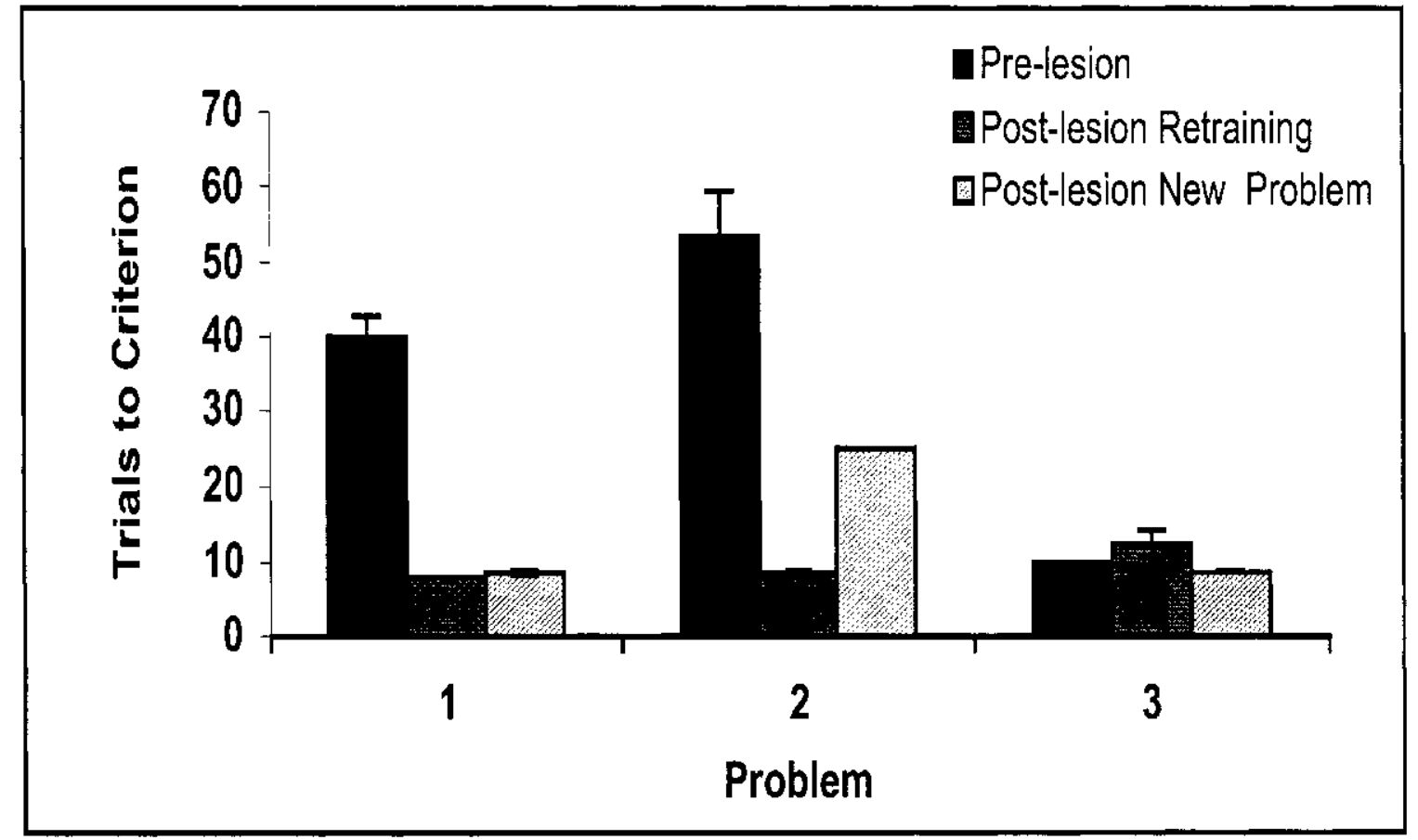

Figure 13. Learning of elemental discriminations occurs at a faster rate after the hippocampal damage. Rats require fewer trials to reach criterion when learning elemental discriminations after the damage to the hippocampus, regardless whether they are the same discriminations that were learned prior to damage (dark gray) or whether they are a completely new set of discrimination problems (diagonal gray stripes). ( $p^{\prime} s<0.05$ ). 


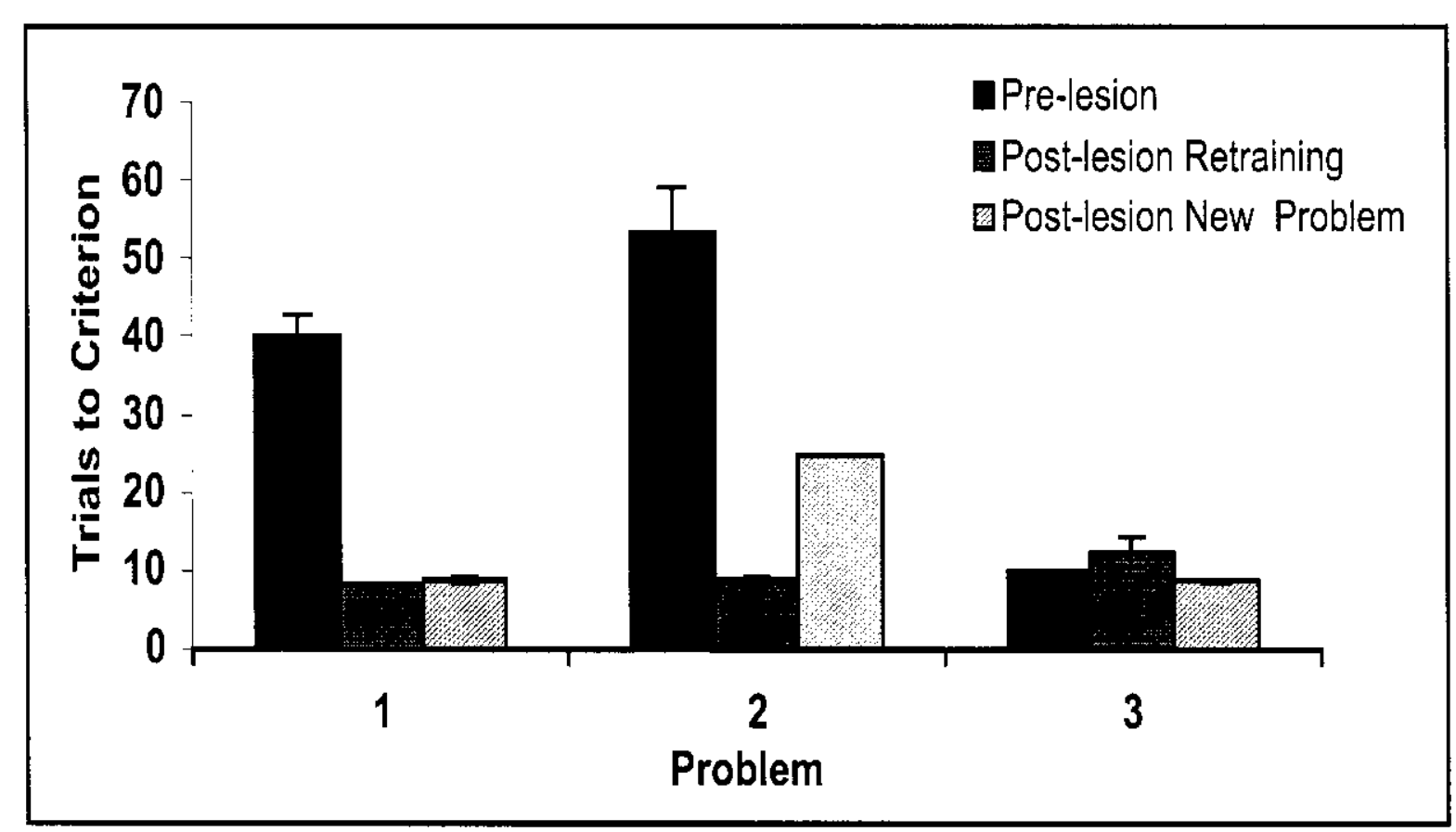

Figure 14. Rats with hippocampal damage exhibit both retrograde and anterograde amnesia for transverse patterning discriminations. When tested after the damage, rats did not remember the transverse patterning problem they learned prior to damage (dark gray) nor were they able to re-learn the problems (diagonal gray stripes). ( $p^{\prime} s<0.05$ ). 


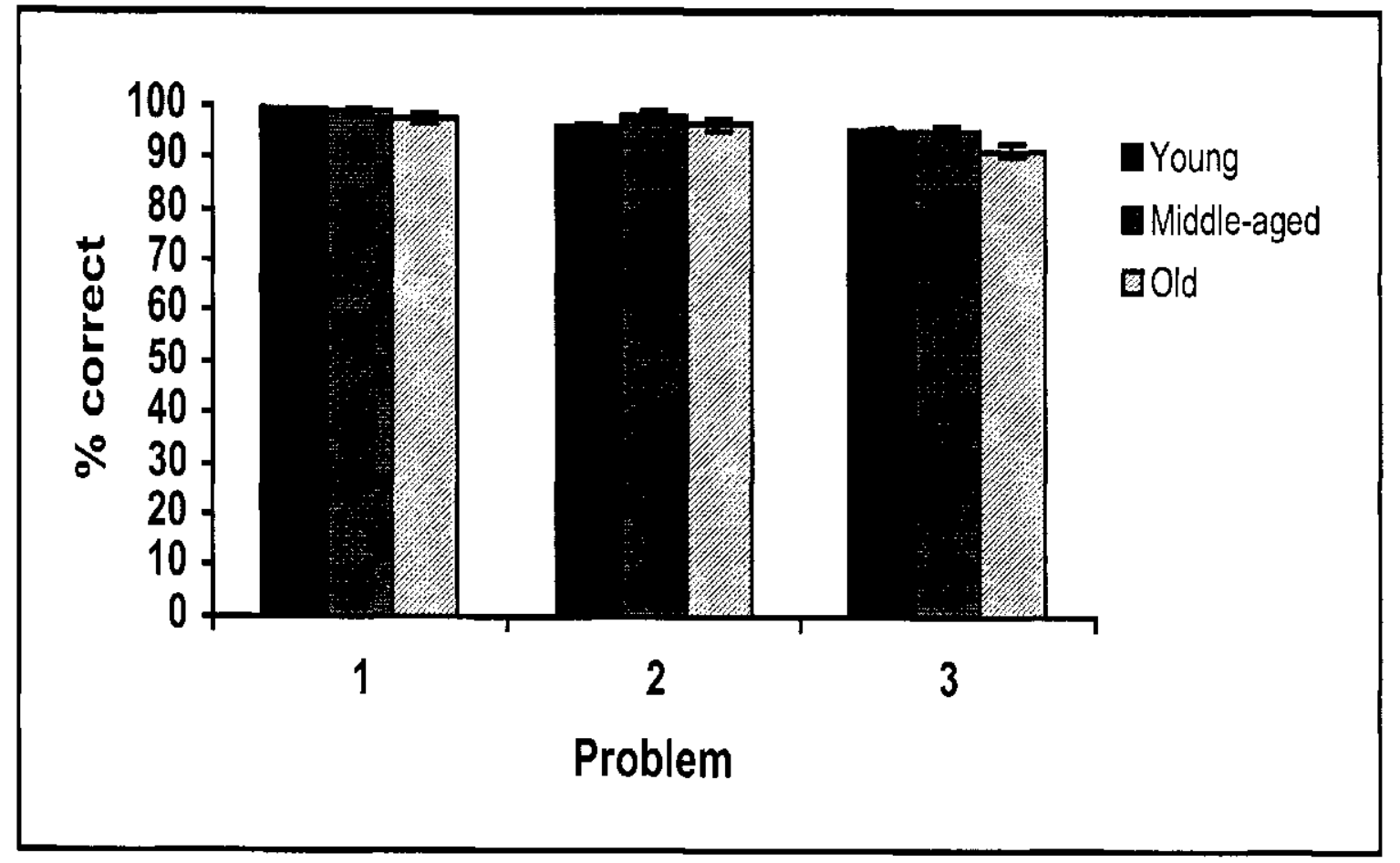

Figure 15. Discriminating between three concurrent elemental problems on the test day. All rats were able to learn elemental discrimination problems and perform with $90 \%$ accuracy or above regardless of their age. Mean and SEM of $\%$ correct for each problem by age group. $(p>.7)$. 


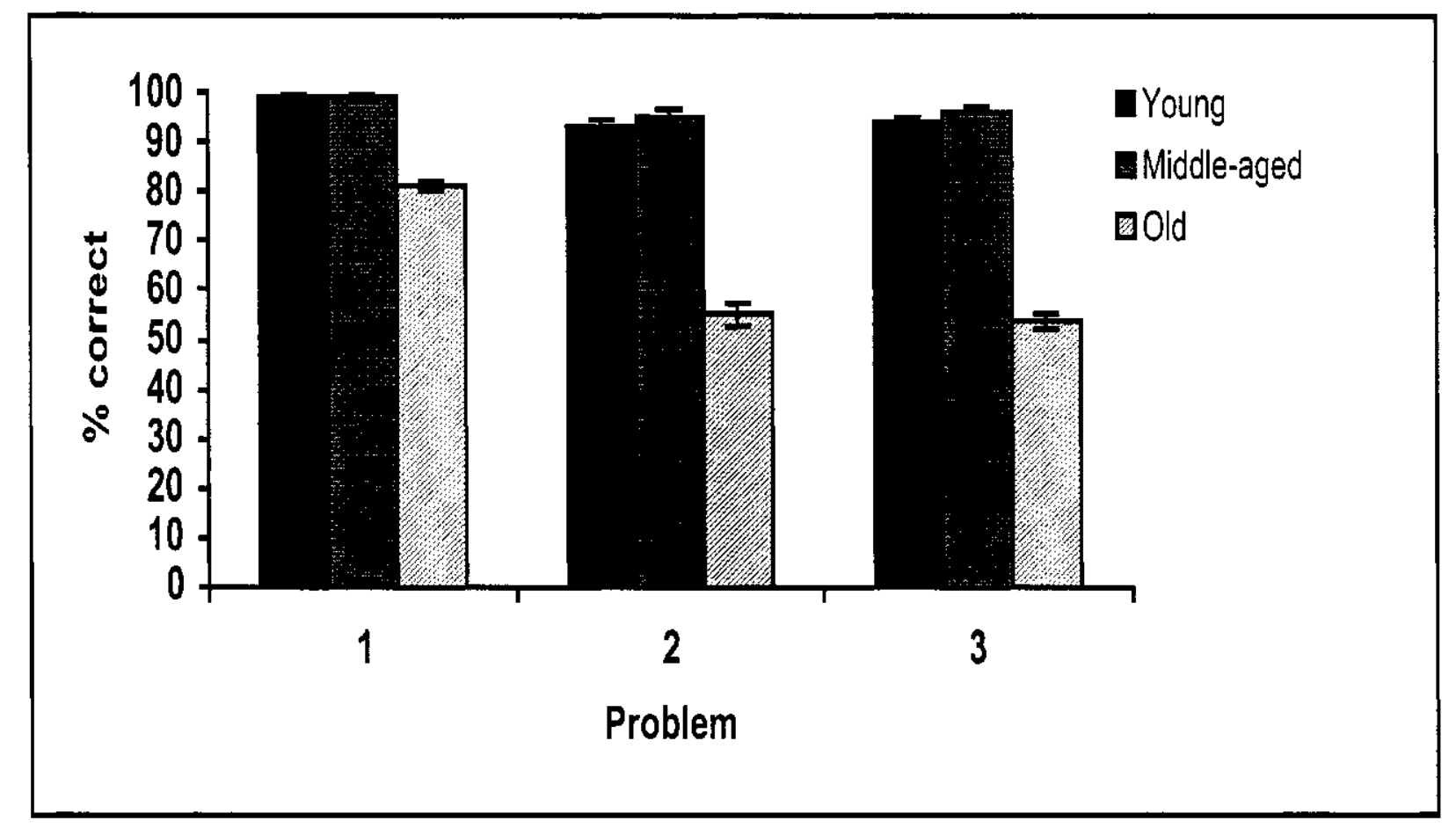

Figure 16. Transverse patterning discrimination performance. Mean and SEM of the $\%$ correct on each of the problems on the test day for each age group. Young and middle-aged rats performed similarly and had no trouble learning the transverse patterning problem, while the old rats were significantly impaired. ( $p$ $<.001$ ). 


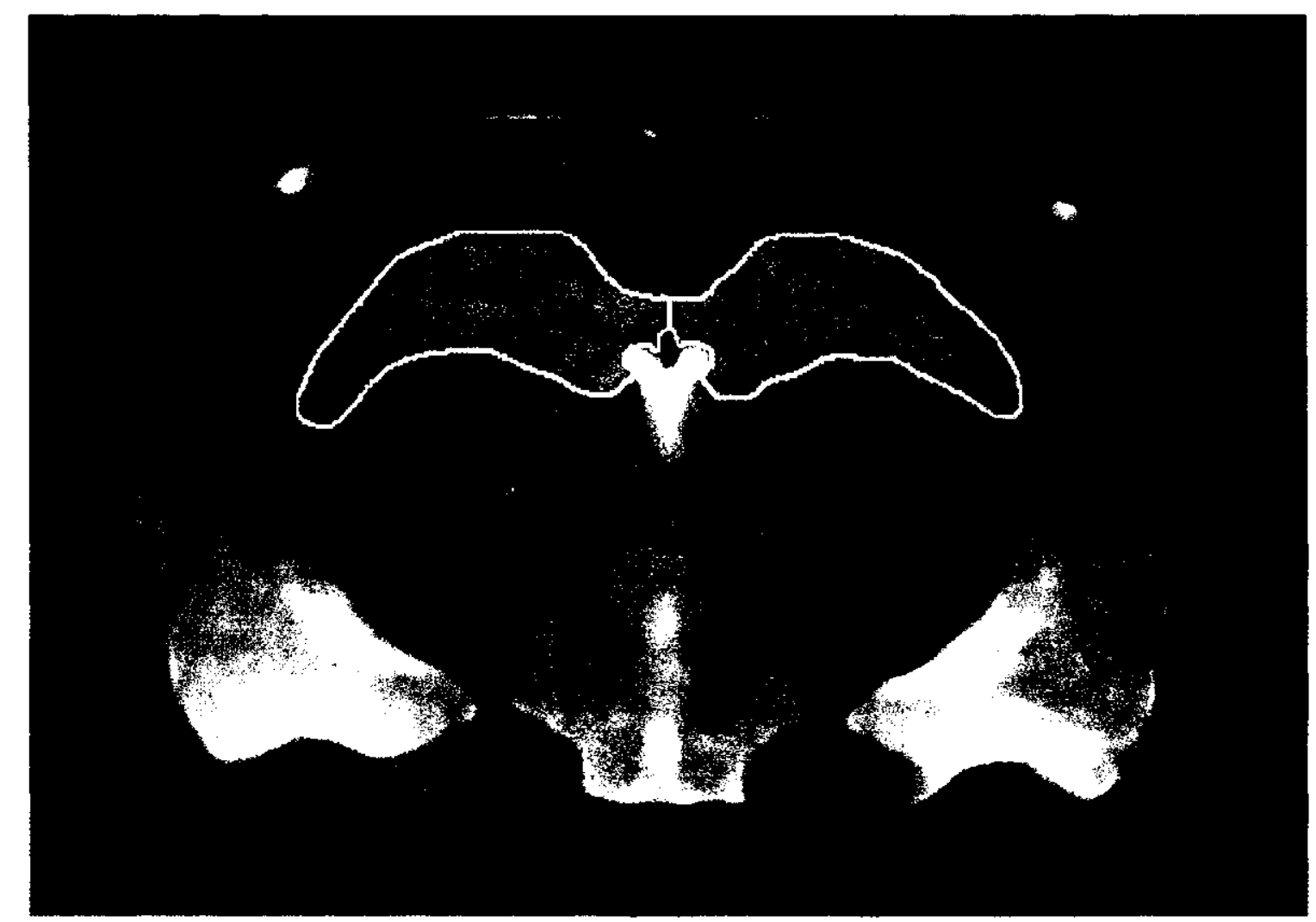

Figure 17. A representative structural $M R$ image (T2) of the dorsal hippocampus of the rat seen here in a coronal plane. Hippocampal area is outlined in white. Structural images were used to obtain a measure of hippocampal volume. 


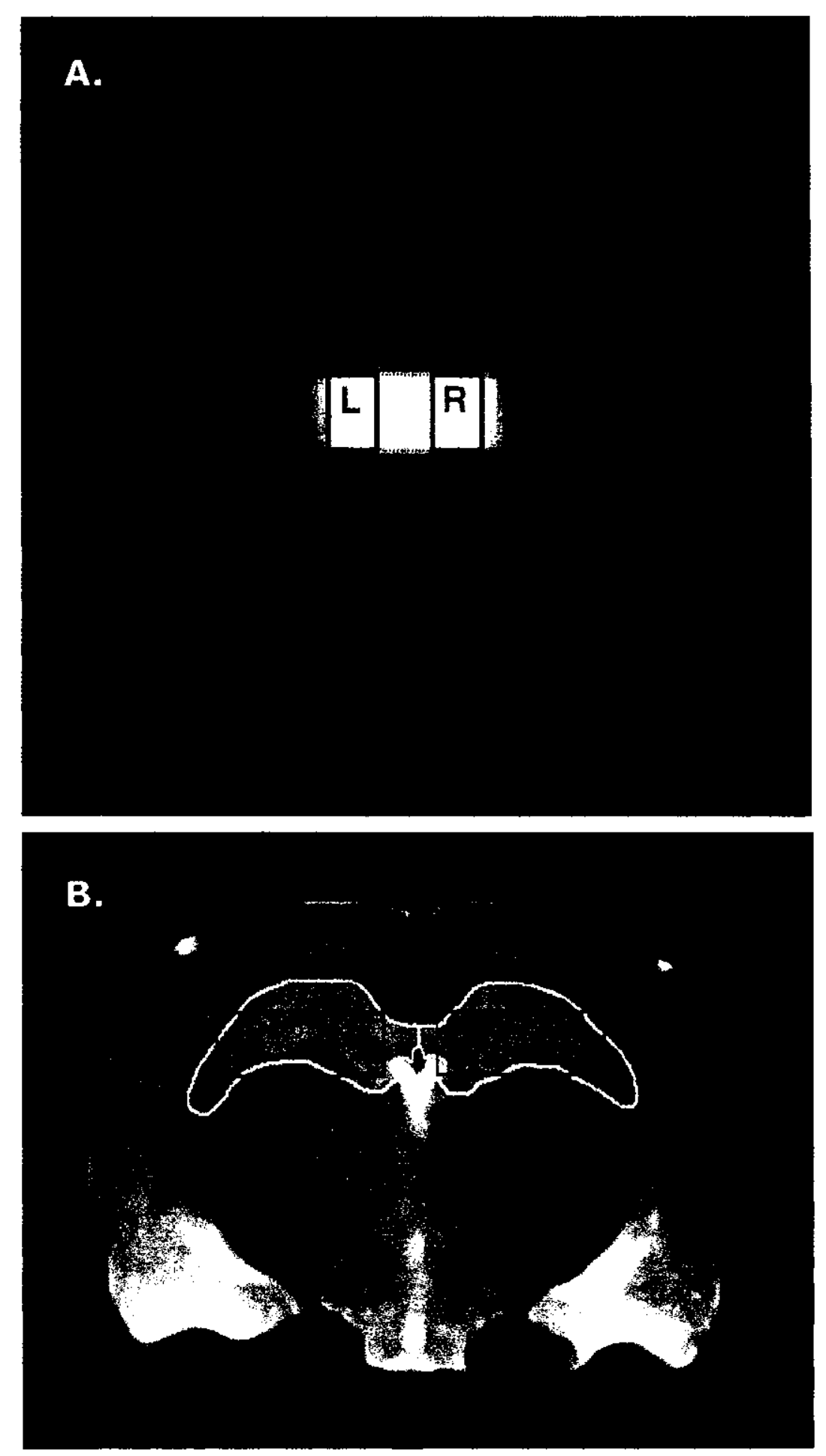

Figure 18. Magnetic Resonance Spectroscopy (MRS) acquisition. Placement of the left $(L)$ and right $(R)$ voxel $(2 \times 2 \times 3)$ in the hippocampus in the (A) axial and $(B)$ coronal plane. 


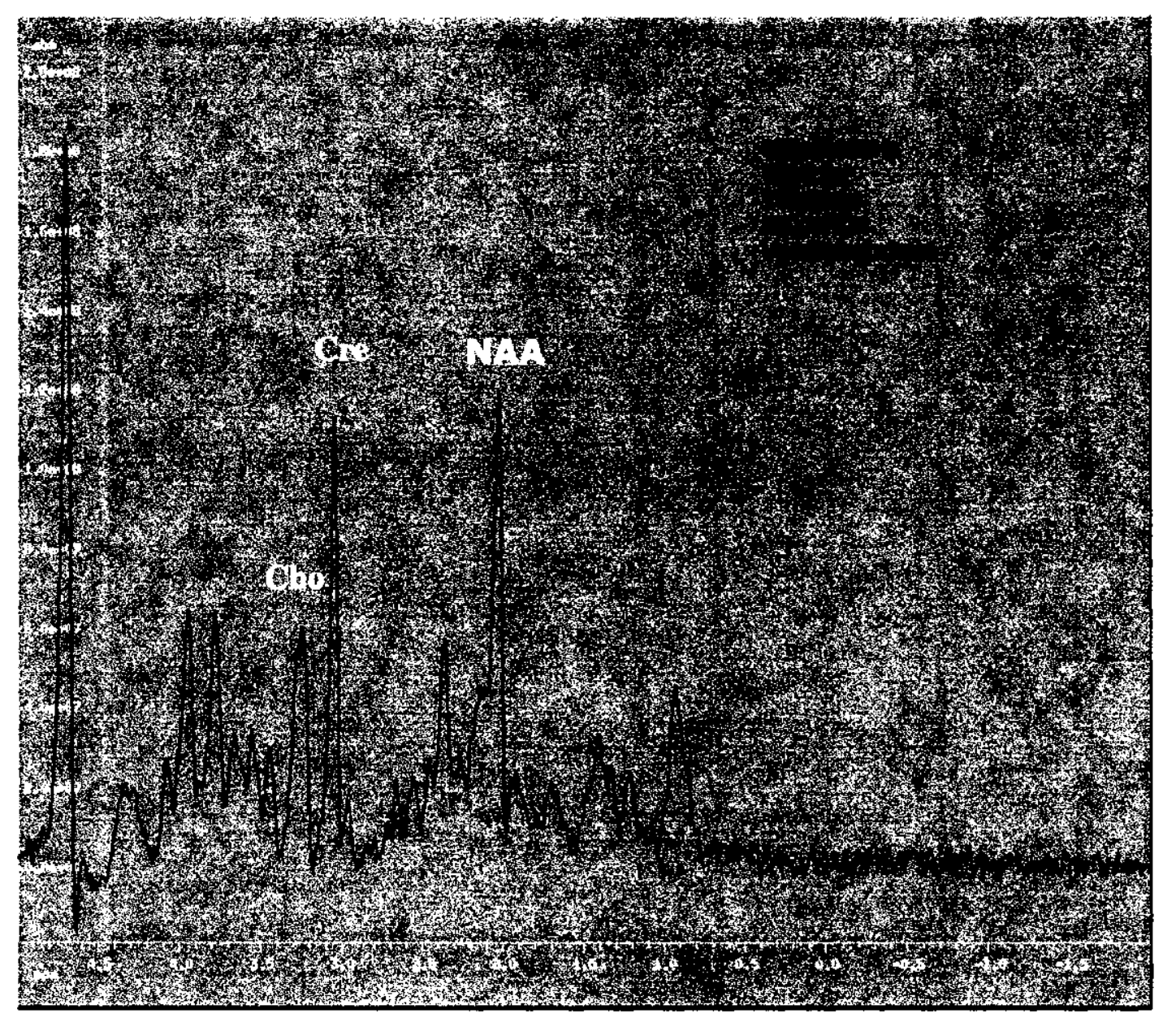

Figure 18B. A representative raw spectrum from the hippocampus at $9.4 \mathrm{~T}$. Three prominent metabolites, Choline, Creatine, and NAA, are of particular interest here. 


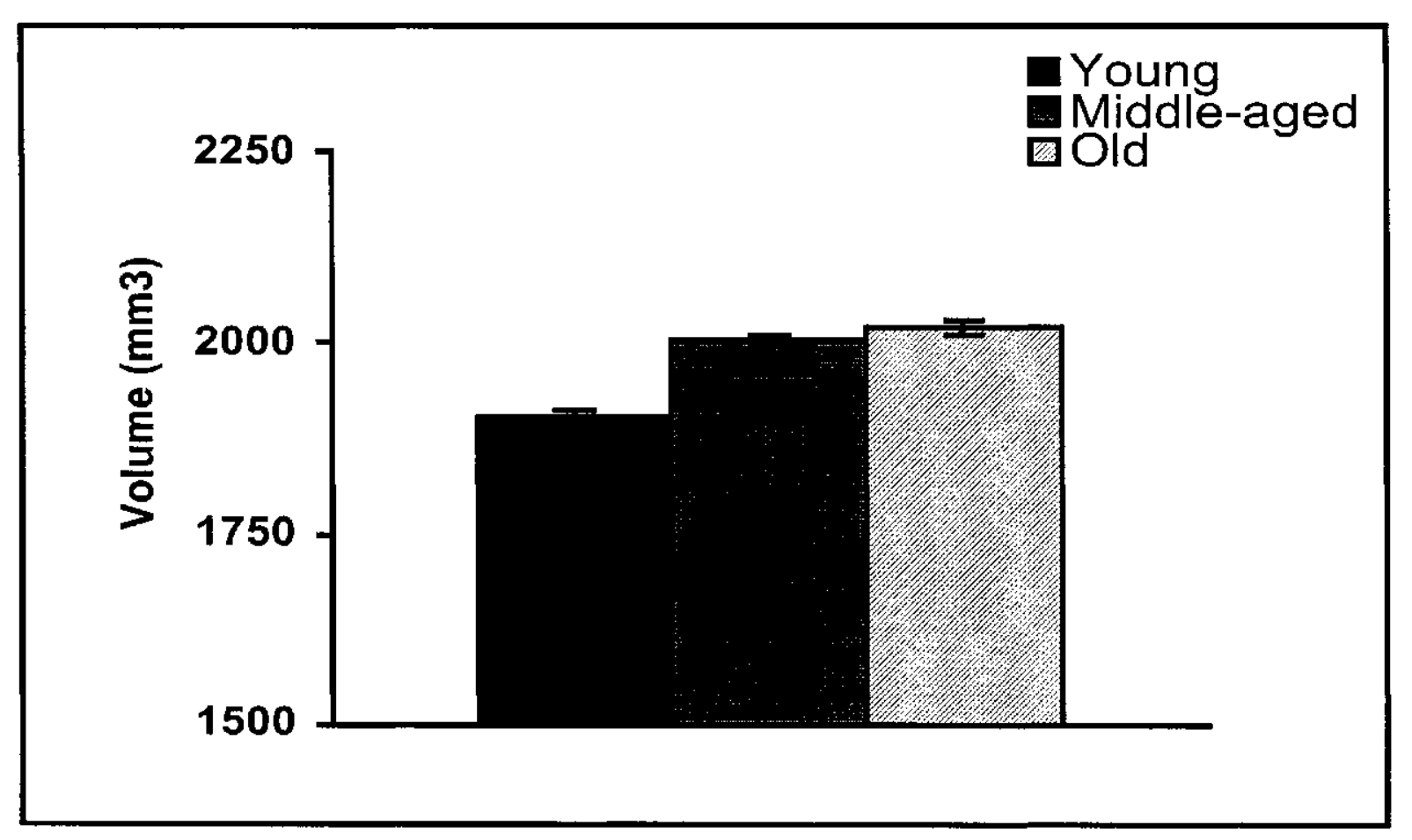

Figure 19. Age-related differences in intracranial volume (ICV). Mean and SEM for ICV are presented for each age group. ICV was significantly smaller in the young rats compared to the middle-aged and old rats $(p<.001)$. 


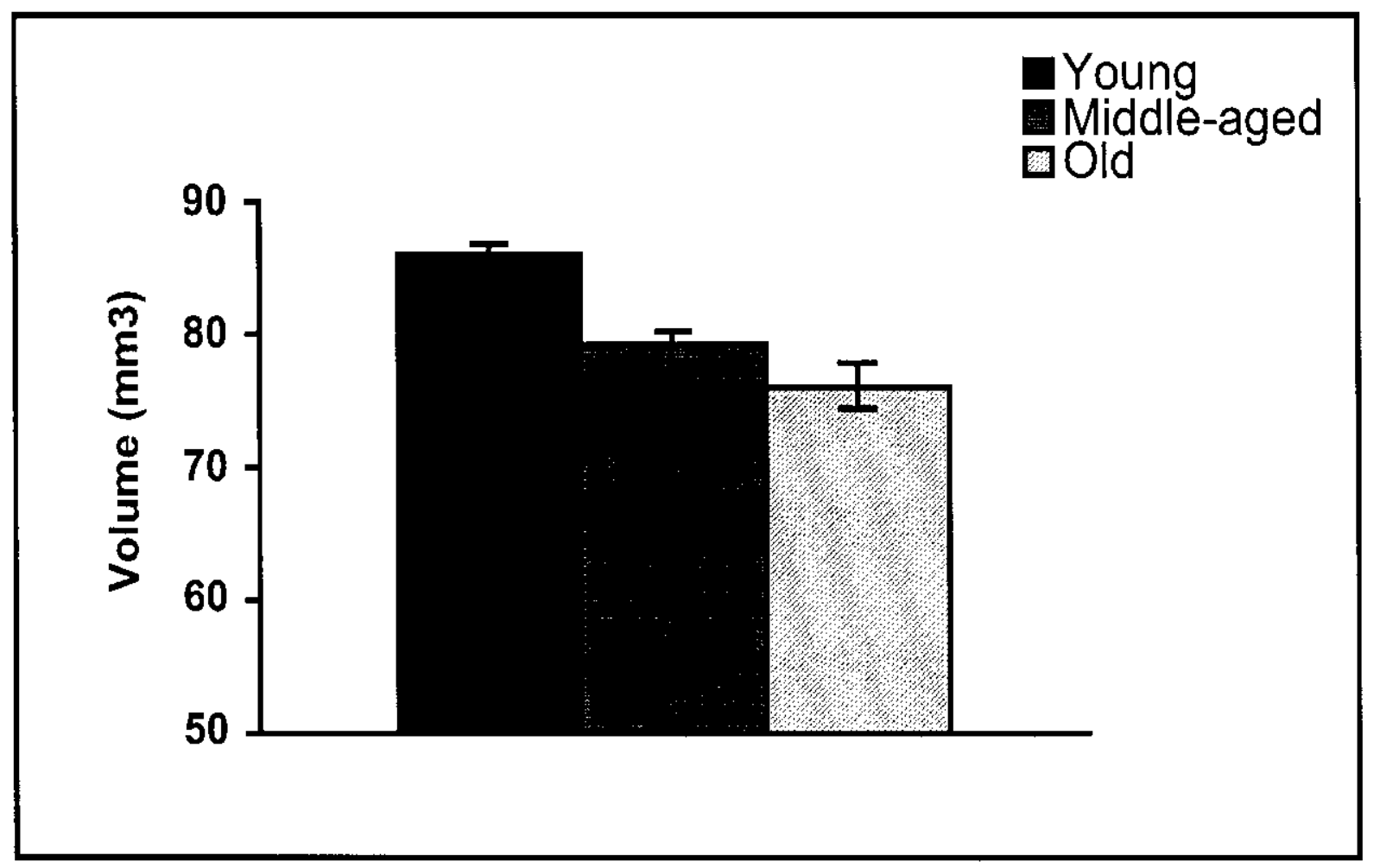

Figure 20. Age-related differences in absolute hippocampal volume. Mean and SEM for the absolute volume of the hippocampus are presented for each age group. Absolute hippocampal volume was significantly smaller in the middleaged and old rats compared to the young rats $(p=.031)$. 


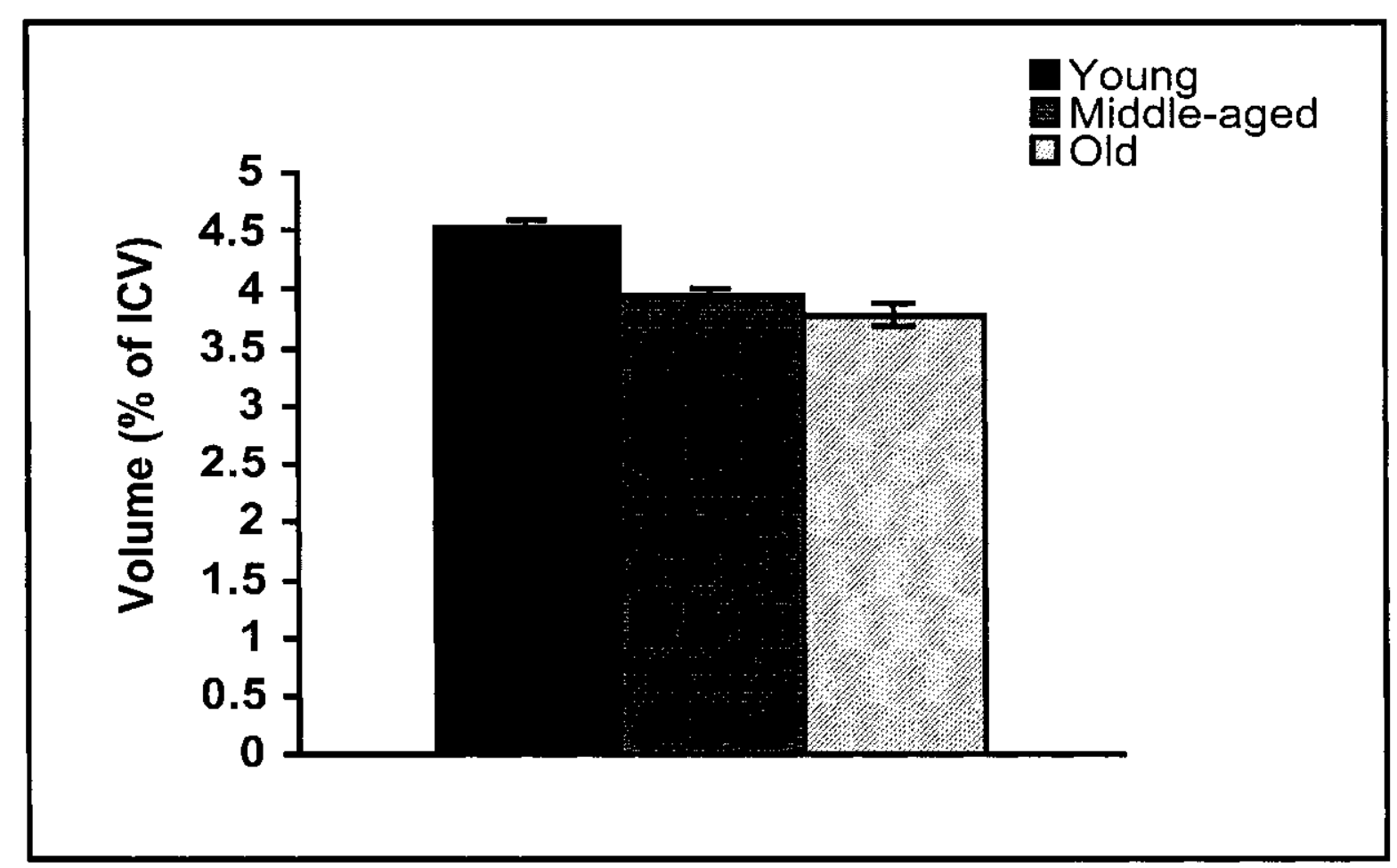

Figure 21. Age-related differences in the hippocampal volume normalized to ICV. Mean and SEM for the normalized hippocampal volume are presented for each age group. Normalized hippocampal volume was significantly smaller in the middle-aged and old rats compared to the young rats $(p=.003)$. 

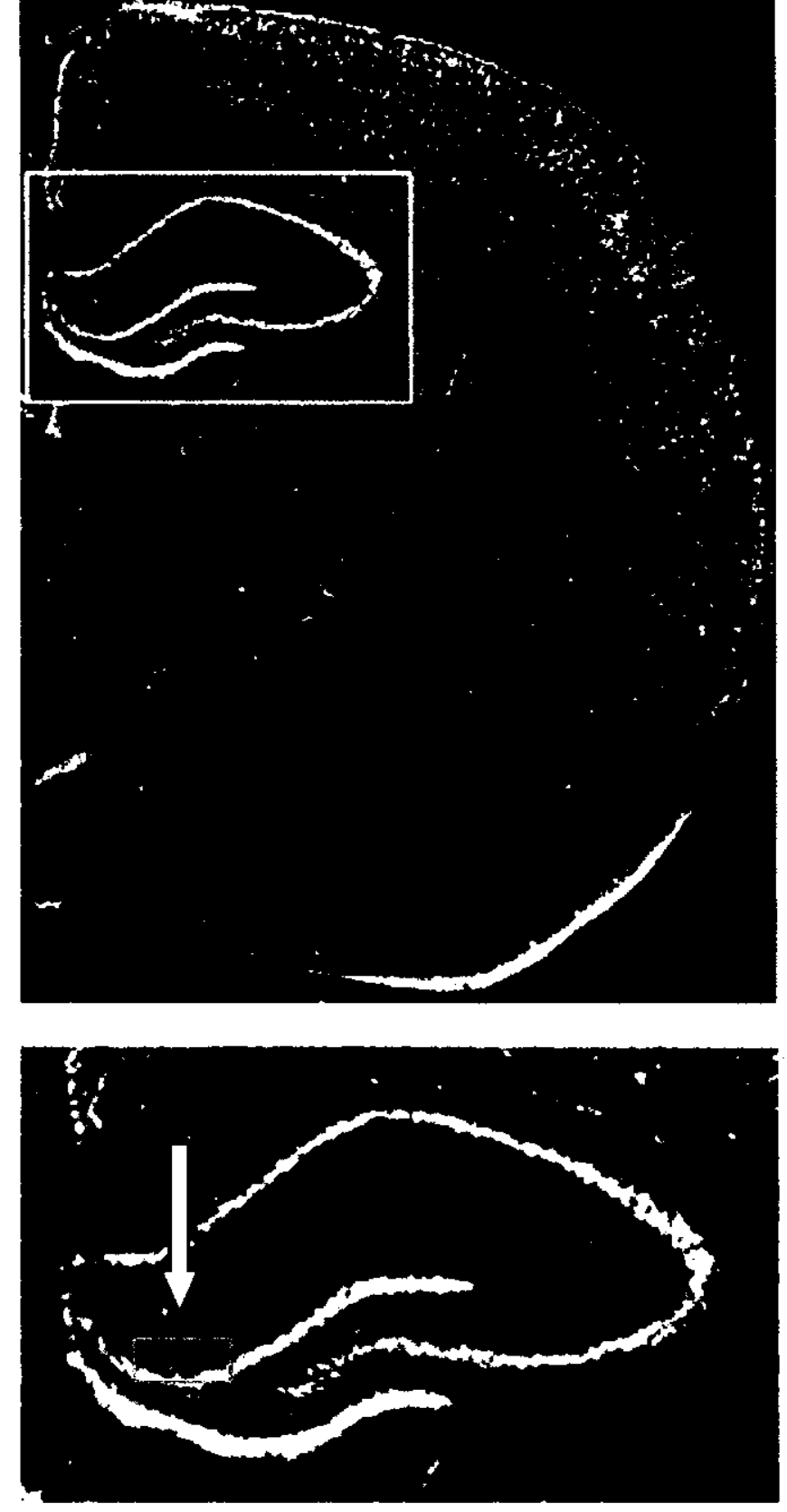

Figure 22. Semi-thin $(1 \mu \mathrm{m})$ sections were obtained from a tissue block stained with toluidine blue for orientation and trimming. Originally, blocks contained the dentate gyrus. The dentate gyrus was then trimmed such that each ultra-thin section spanned the inner molecular layer as well as the very dorsal portion of the granule cell layer (see arrow pointing to a light blue rectangle). A series of ultra-thin section for electron microscopy were obtained from the trimmed blocks. 


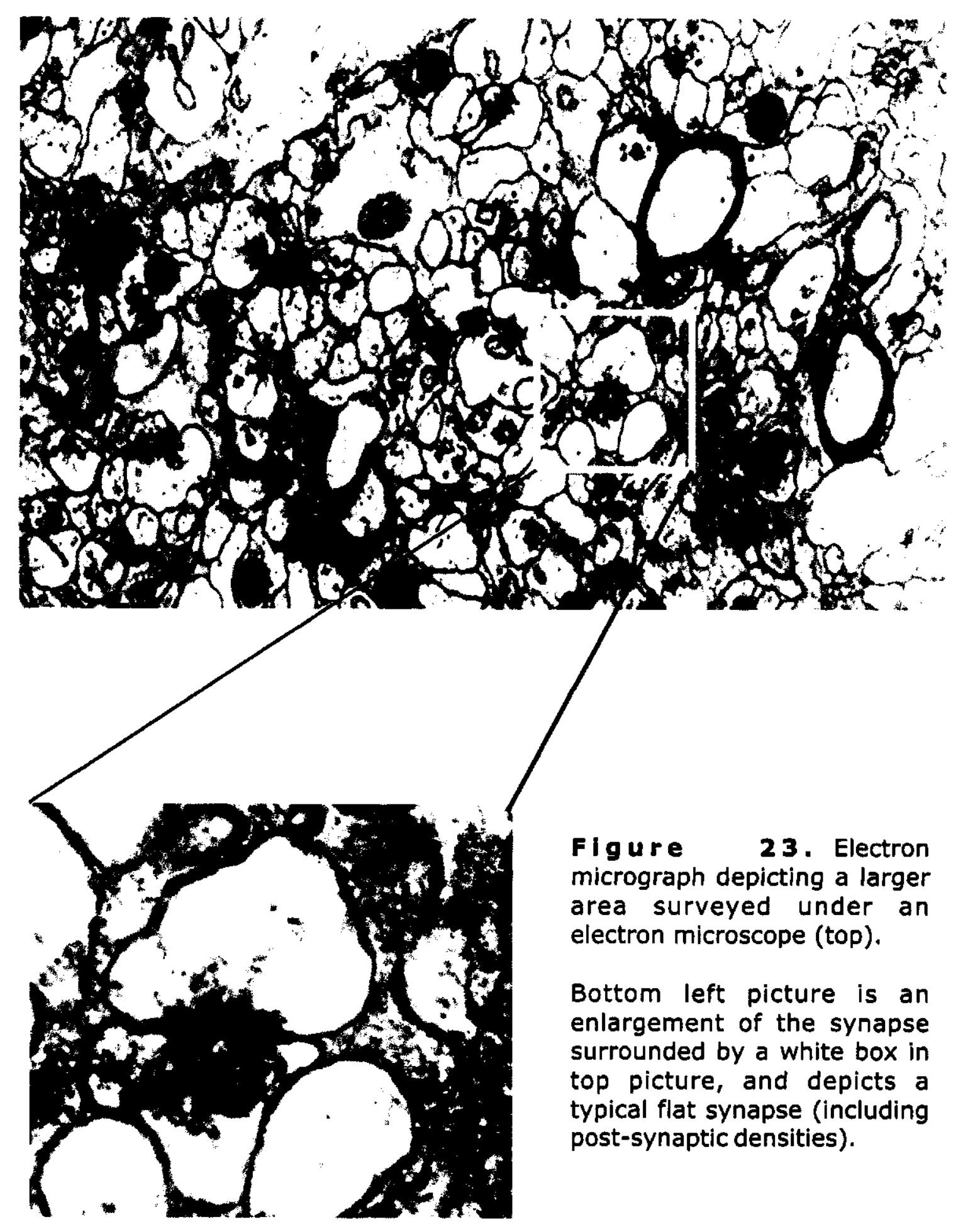




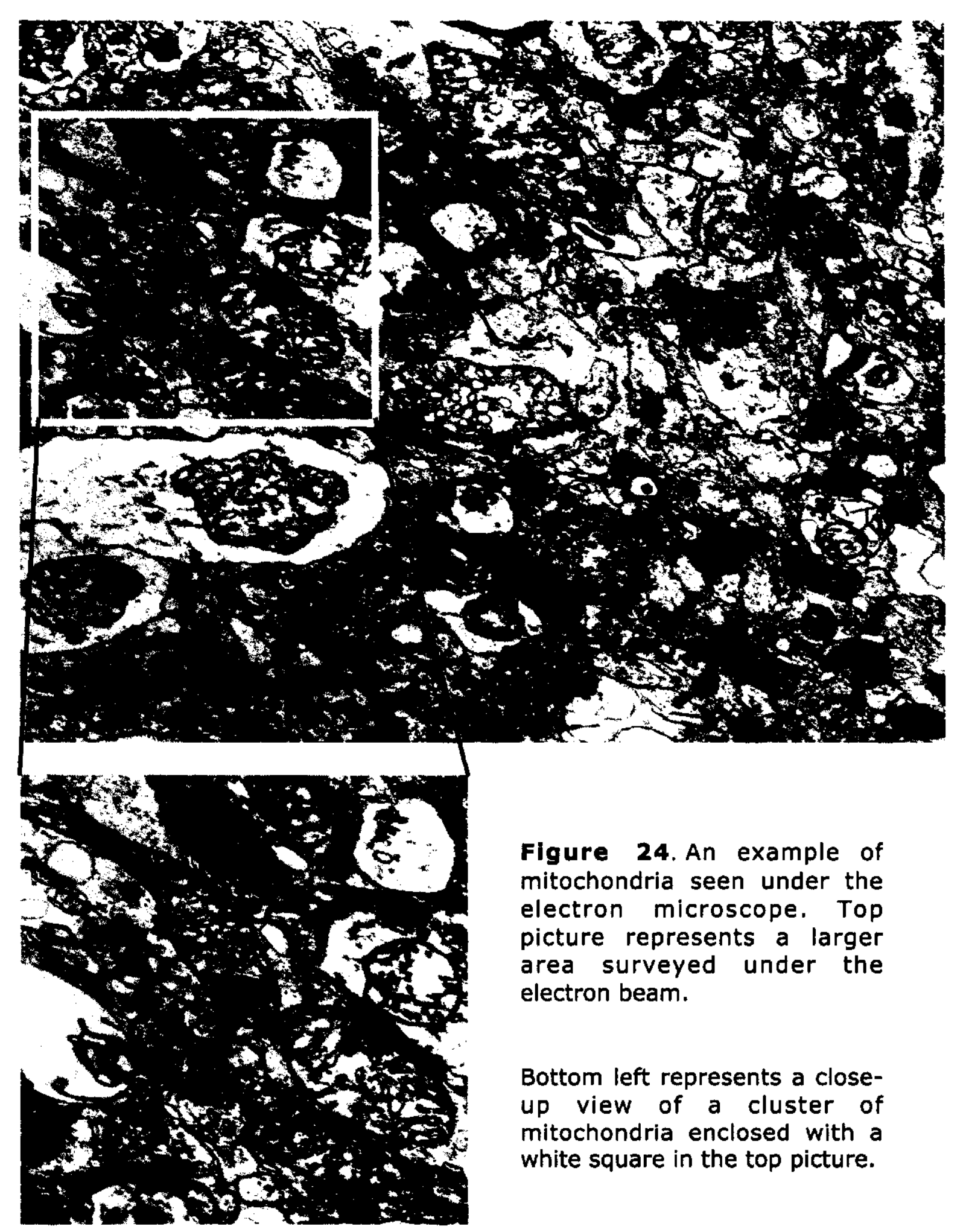




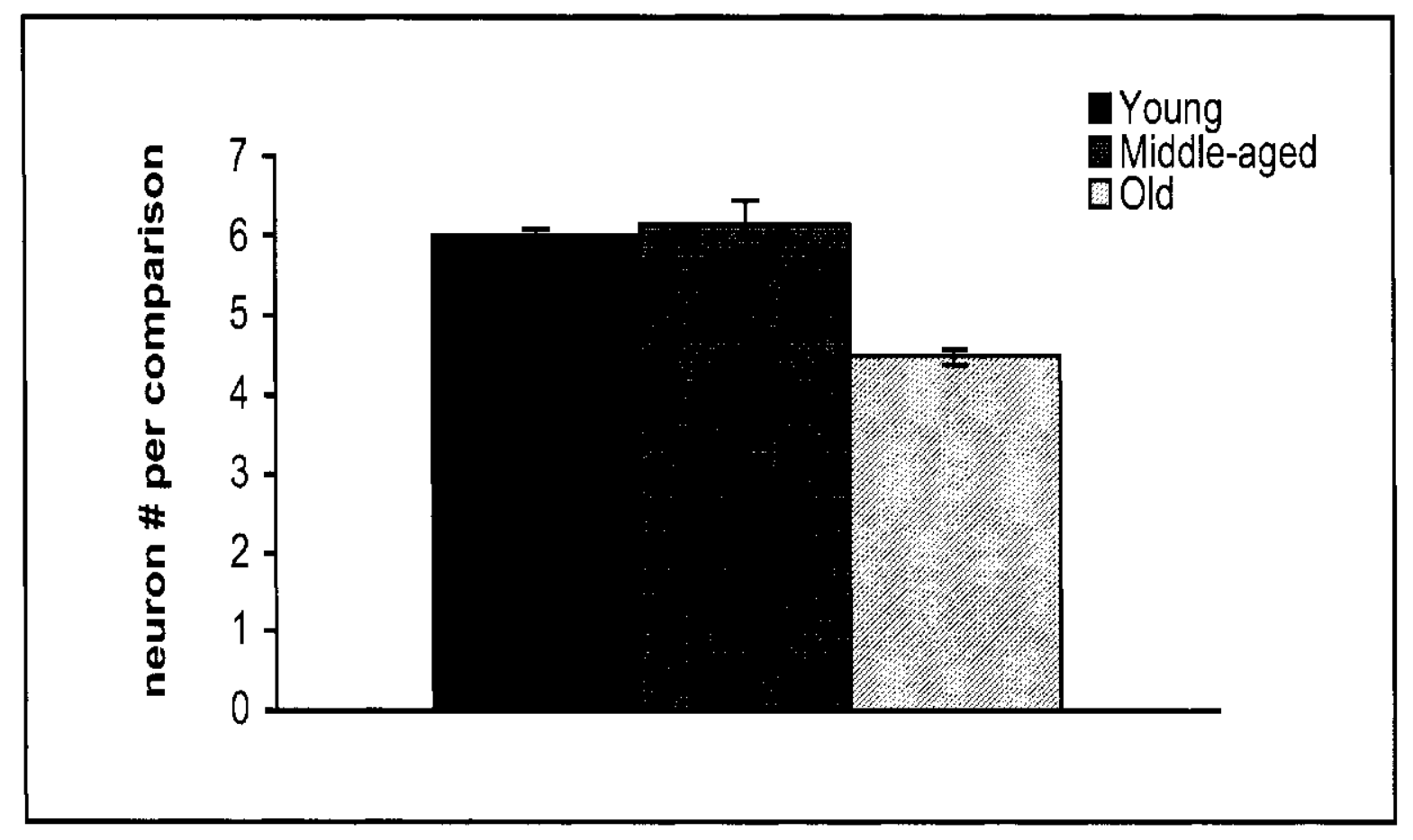

Figure 25. Changes in neuronal density in the inner molecular layer (IML) of the hippocampus. Mean and SEM for neuronal density within the IML. Neuronal density was similar in the young and middle-aged rats, but was significantly lower in the old rats $(p=.019)$. 


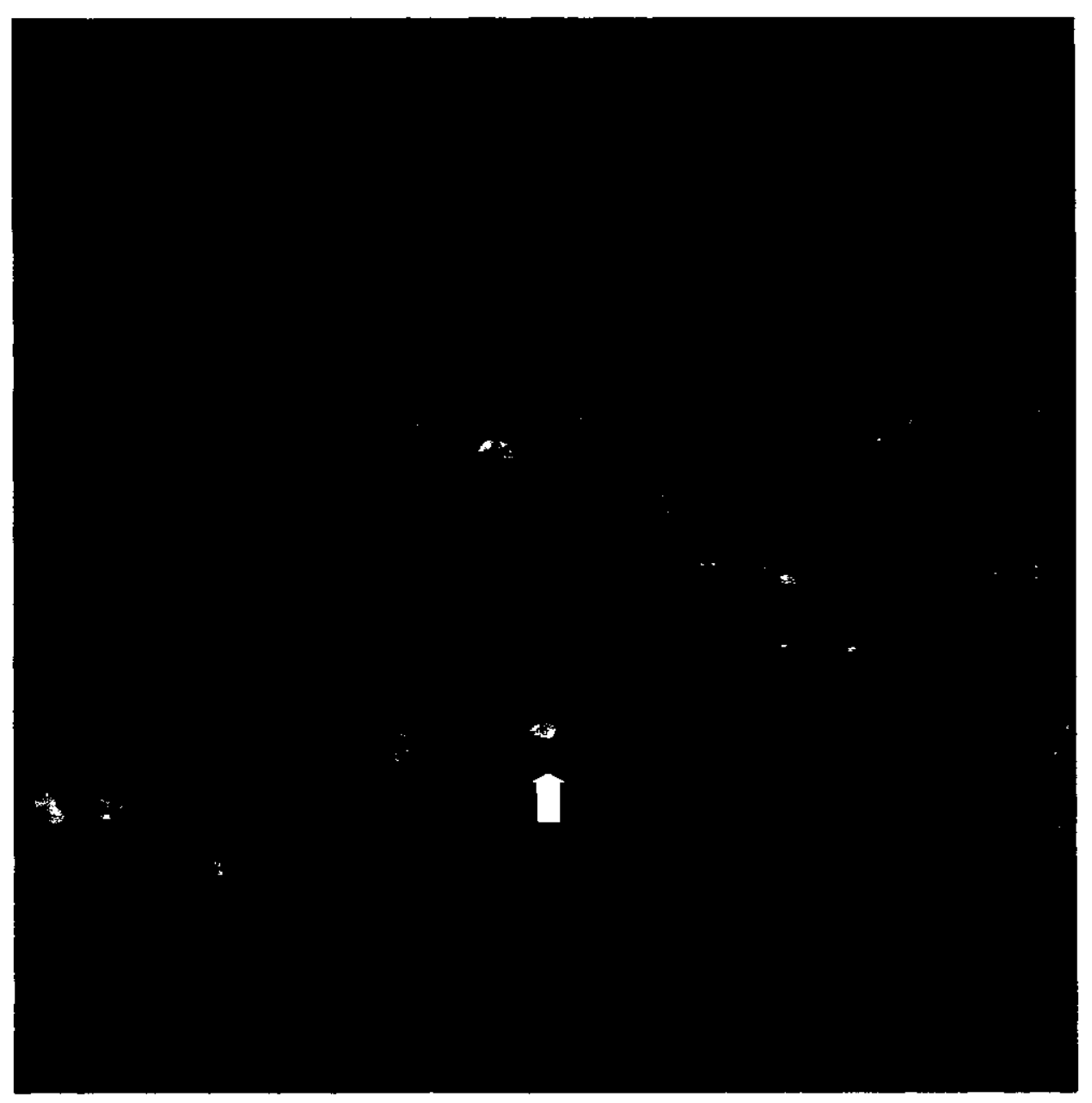

Figure 26. Newly born neurons in the dentate gyrus. A confocal image of neurons in the granule cell layer double-labeled against antibodies for BrdU (red) and NeuN (green). Five weeks after the last injection of BrdU, cells double-labeled for BrdU and NeuN can be detected. This image shows (consistent with previous findings) that many BrdU/NeuN-labeled cells, indistinguishable from the surrounding granule cells can be found 5 weeks after BrdU administration. White arrow is pointing to a 'gold' cell, a cell that is showing an immunoreaction for NeuN in a BrdU-labeled cell. 
Figure 27. Doublecortin labeling in the dentate gyrus of the hippocampus (young animal shown). DCX-labeled cells are restricted to the subgranular zone (SGZ). DCX-expressing cells often have long processes that extend into the molecular layer. The black arrow is pointing to three DCX-labeled cells situated in the SGZ along the ventral horn of the dentate gyrus with long processes extending into the molecular layer. 


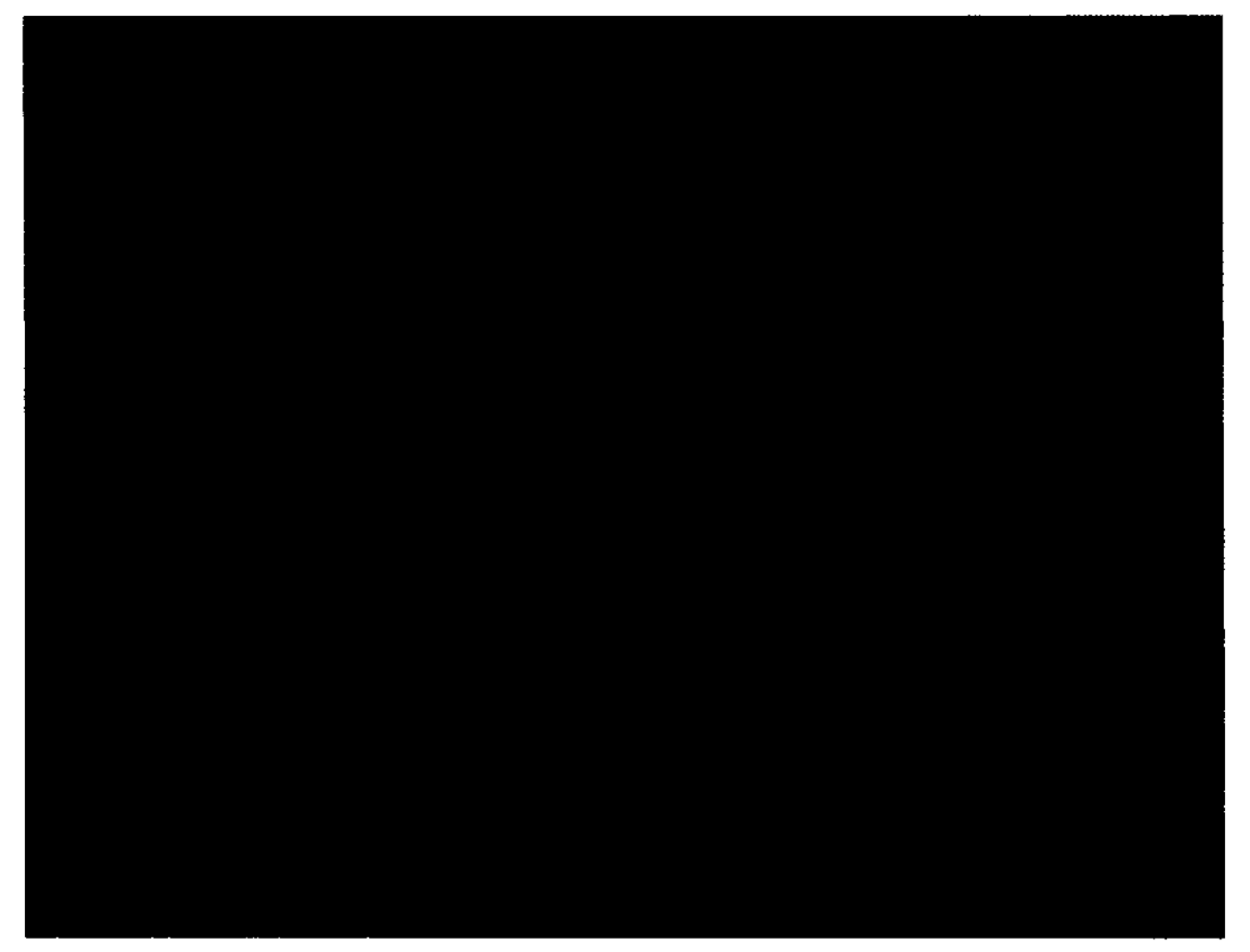

Figure 28. Newly born neurons expressing Ki67 along the subgranular zone in the dentate gyrus of a representative young rat. Ki67 is expressed in cycling cells and here is fluorescing in red along the dentate gyrus. 


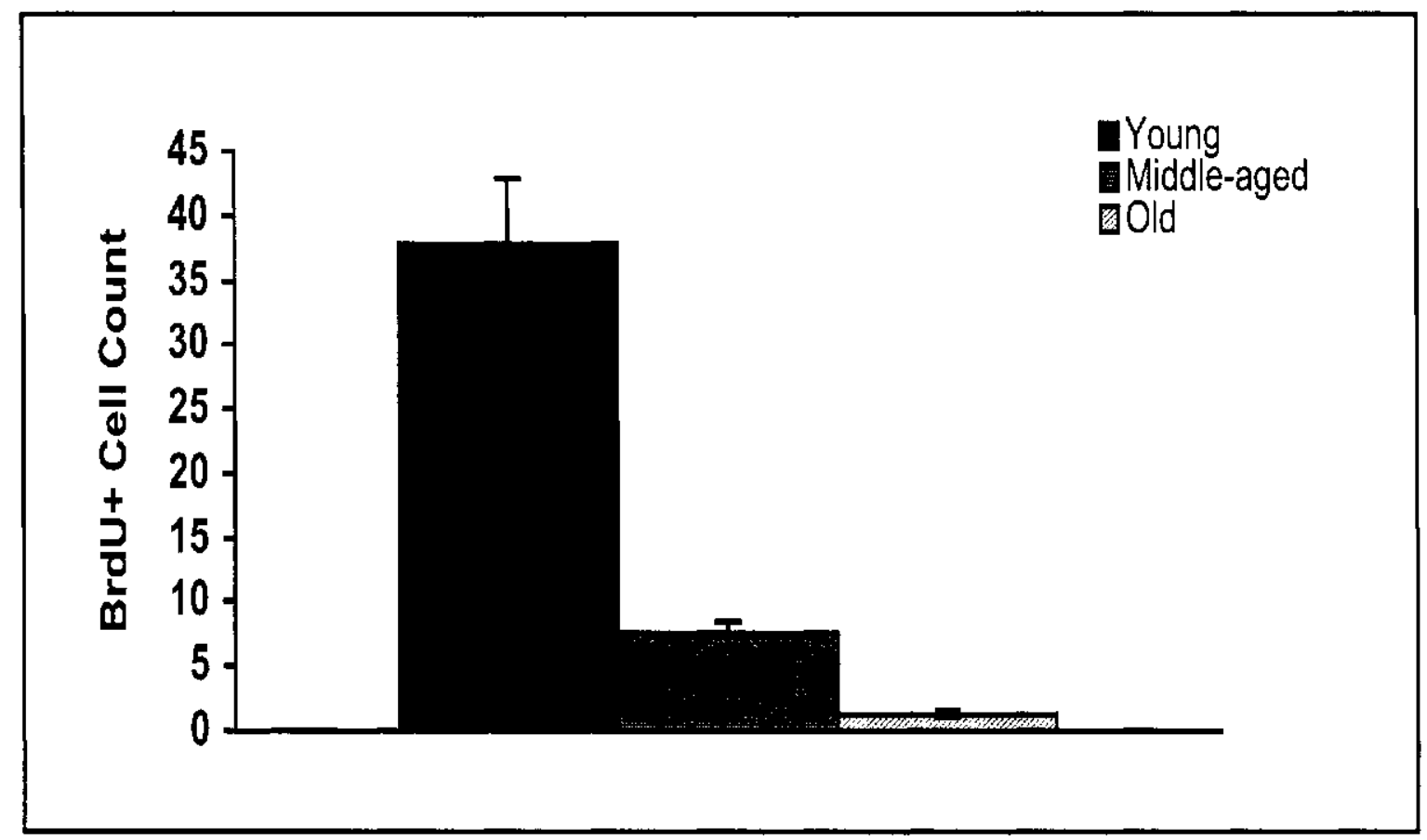

Figure 29. The number of BrdU labeled cells. Mean and SEM by age group for the total number of BrdU-labeled cells present 5 weeks after the BrdU administration. The number of BrdU labeled cells significantly differed between each age group $(p=.002)$. 


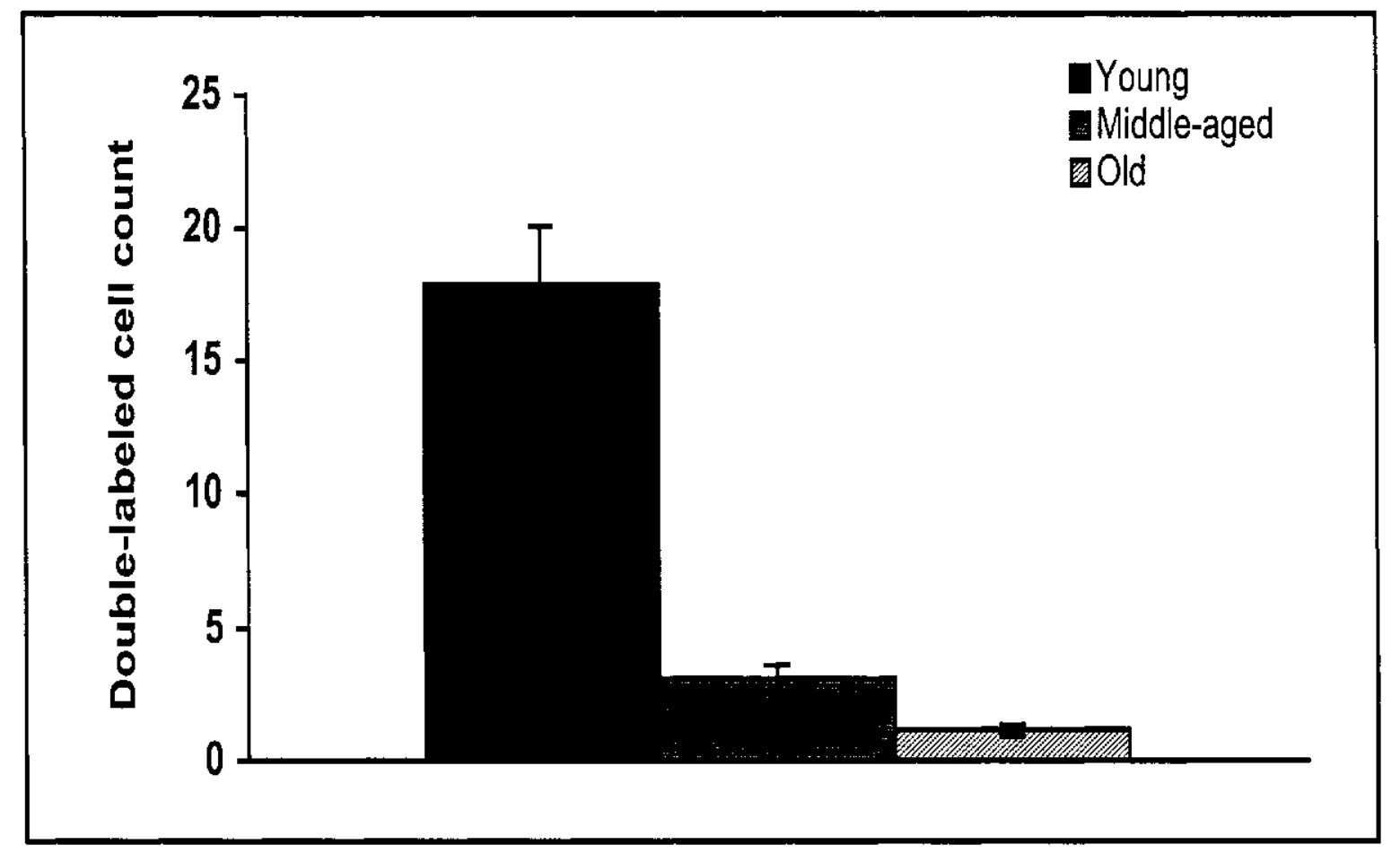

Figure 30. The number of BrdU labeled cells that are also co-labeled with NeuN (a mature neuronal marker). Mean and SEM by age group for the total number of BrdU/NeuN double-labeled cells present 5 weeks after the BrdU administration. The number of BrdU/NeuN double-labeled cells significantly differed between each age group and decreased with age $(p=.001)$. 


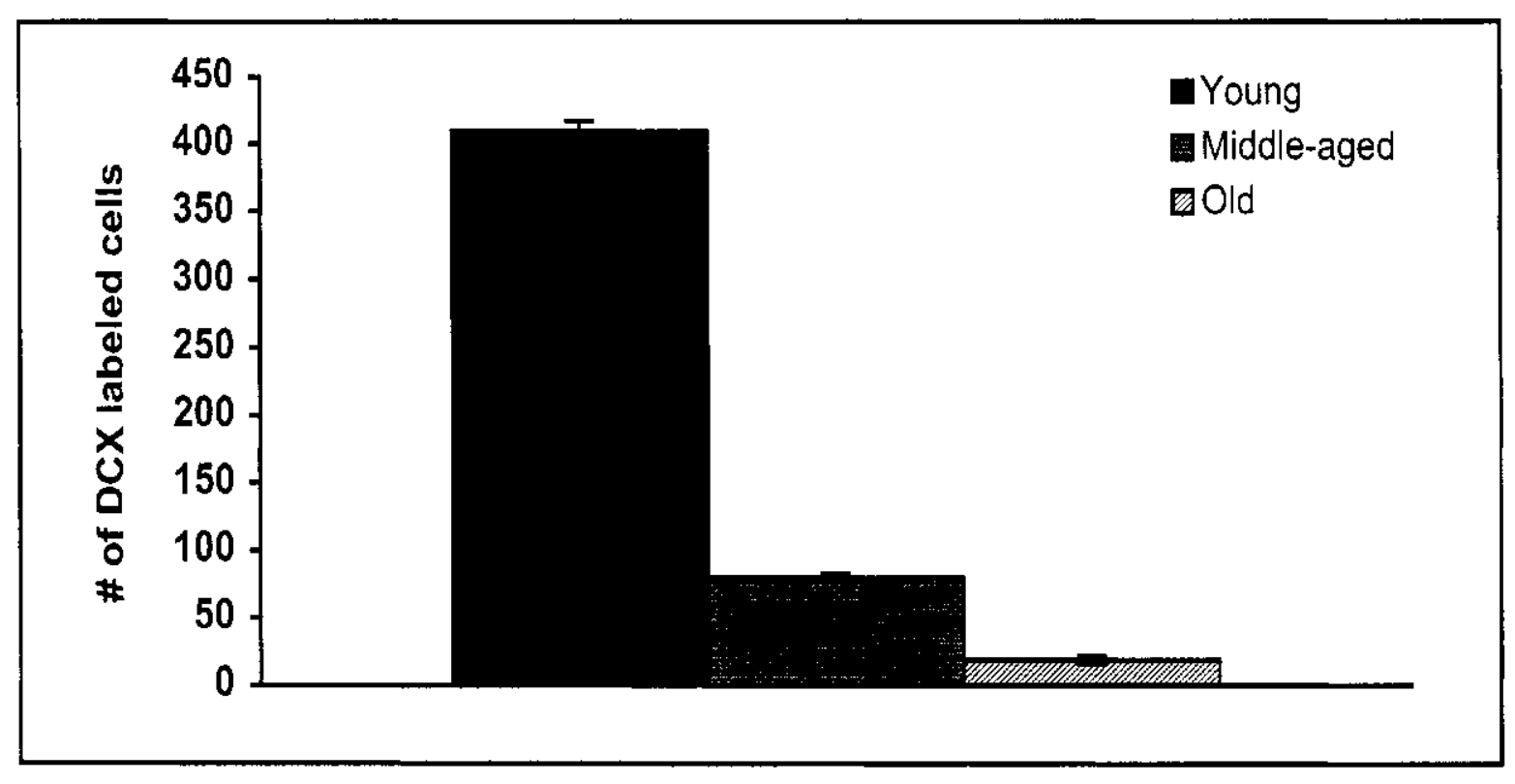

Figure 31. The number of cells labeled with $D C X$, a marker of migrating neuroblasts (immature neurons). Mean and SEM by age group for the total number of DCX-labeled cells. The number of DCX-labeled cells significantly differed between each age group and decreased with age $(p<.001)$. 


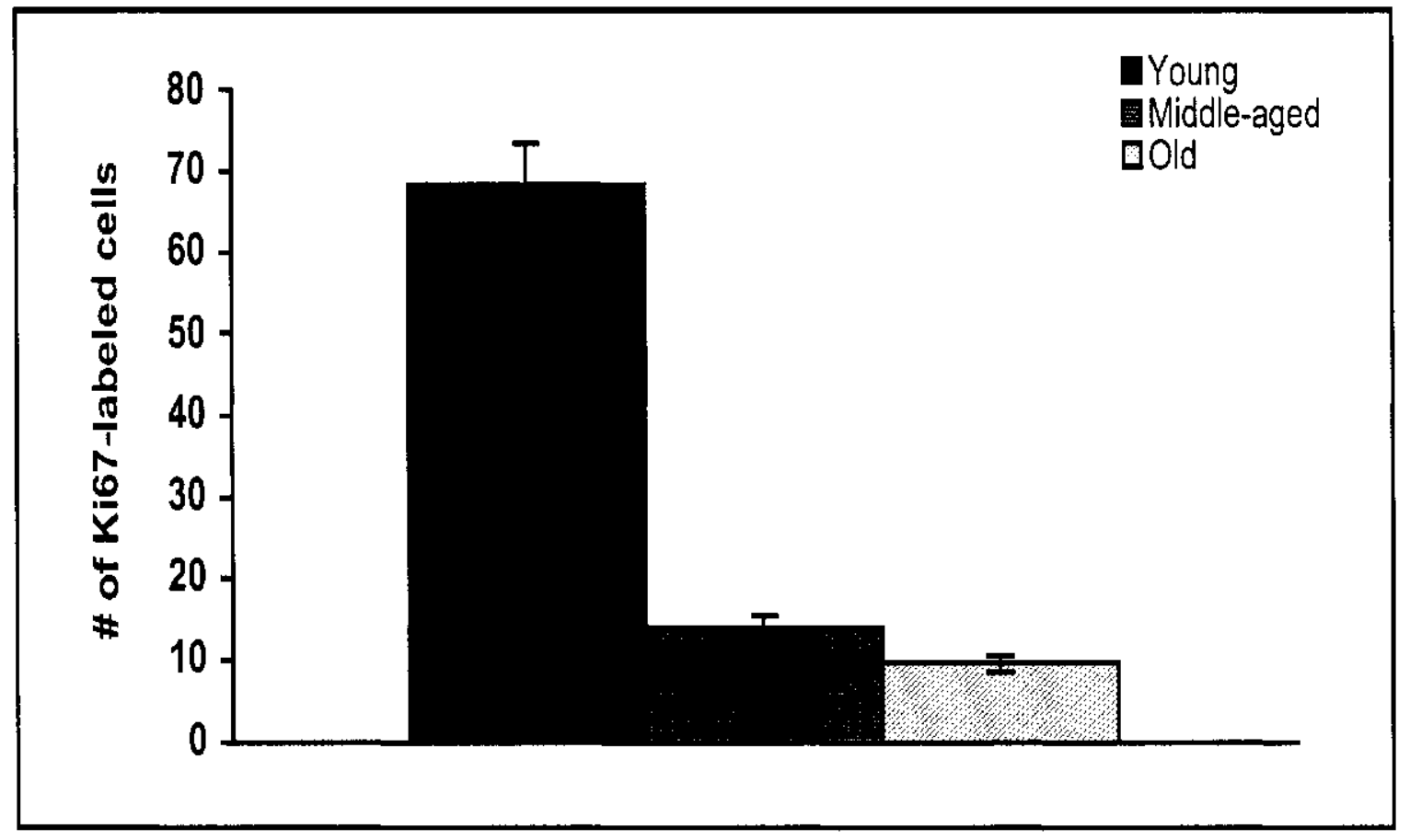

Figure 32. The number of cells labeled with Ki67, a marker for cycling cells. Mean and SEM by age group for the total number of Ki67-labeled cells. The number of Ki67-labeled cells decreased with age, such that both middle-aged and old rats had a significantly lower number of Ki67+ cells, but the two groups did not differ from one another $(p<.001)$. 


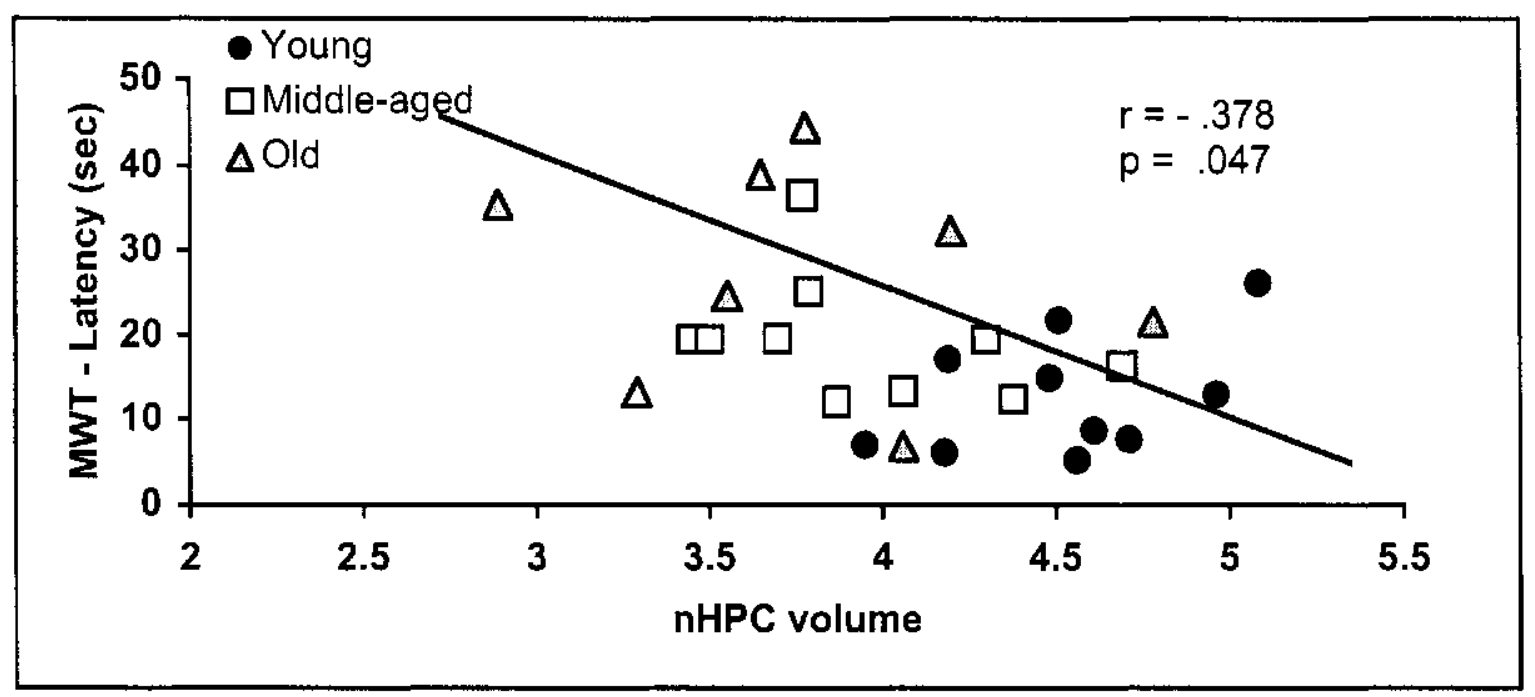

Figure 33. Correlations between performance of young (black circles), middleaged (open squares), and elderly (gray triangles) rats on the MWT with normalized hippocampal (nHPC) volume. Larger hippocampal volume was correlated with shorter approach latencies on the first trial across the days the platform remained in the same location as the previous day $(p=.047)$. 


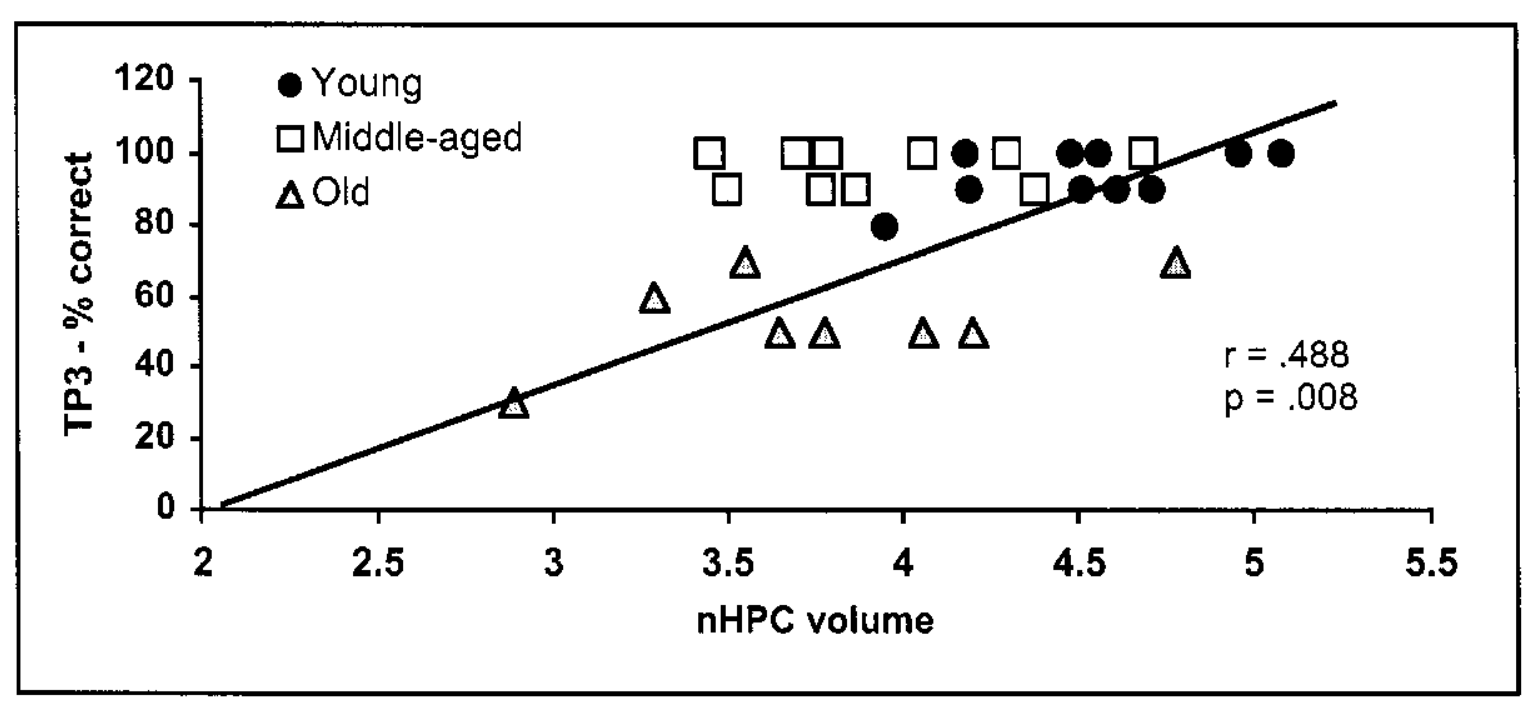

Figure 34. Correlations between performance of young (black circles), middleaged (open squares), and elderly (gray triangles) rats on the transverse patterning task with normalized hippocampal (nHPC) volume. Larger hippocampal volume was correlated with better performance on Problem 3 of the transverse patterning task. 


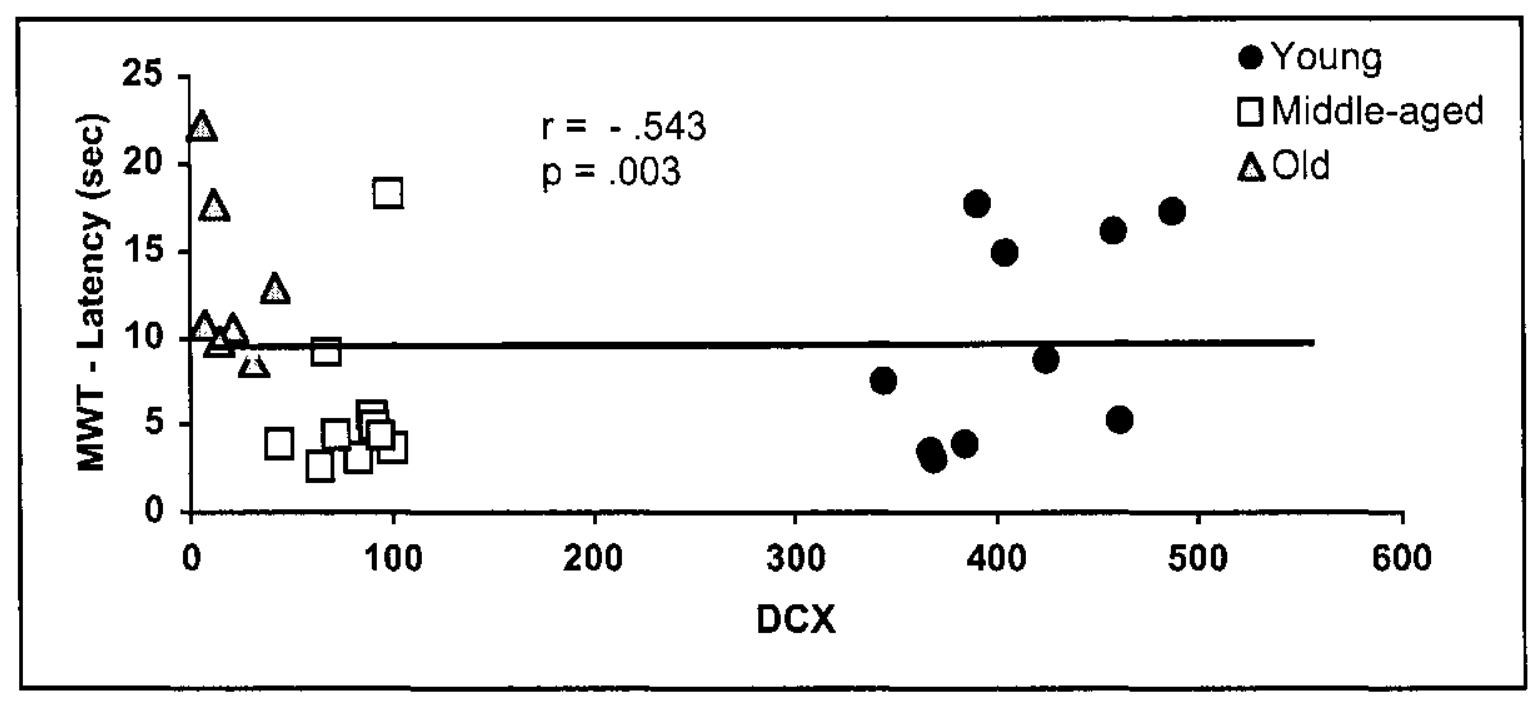

Figure 35. Correlations between performance of young (black circles), middleaged (open squares), and elderly (gray triangles) rats on the MWT with the number of DCX-labeled cells. Shorter latencies in locating the platform the last trial across the days the platform was in a new location significantly correlated with a larger number of DCX-labeled cells. However, this relationship was significant in the senescent rats (middle-aged and old combined; $r=-.534, p=$ .027 ), but in the young group (and it is in the opposite direction; $r=.506, p=$ $.136)$. 


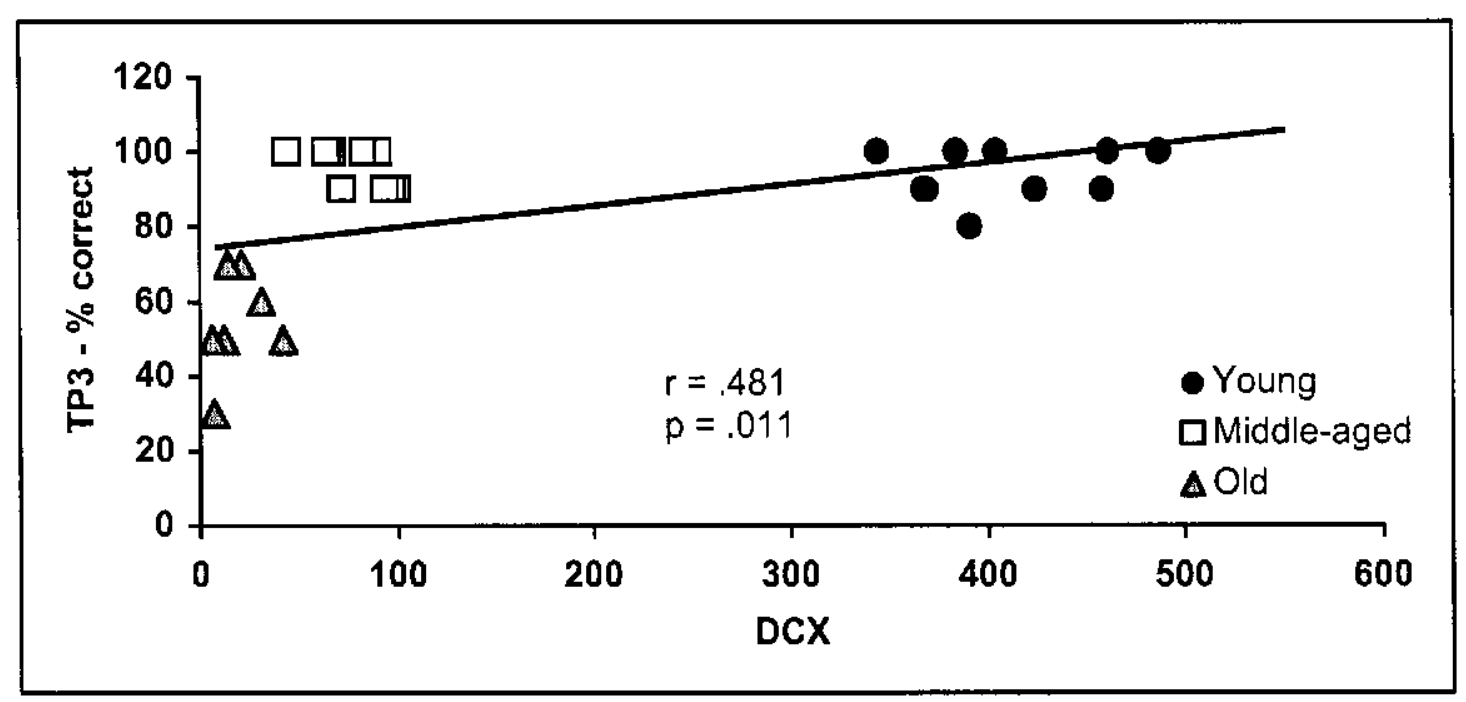

Figure 36. Correlations between performance of young (black circles), middleaged (open squares), and elderly (gray triangles) rats on the transverse patterning task with the number of DCX-labeled cells. Better performance on Problem 3 of the transverse patterning task significantly correlated with a larger number of DCX-labeled cells. However, the relationship between DCX and performance on Problem 3 of the transverse patterning task was significant in the elderly (middle-aged and old together; see Figure $36 ; r=.805, p<.001$ ), but not in the young rats $(r=.180, p=.619)$. 


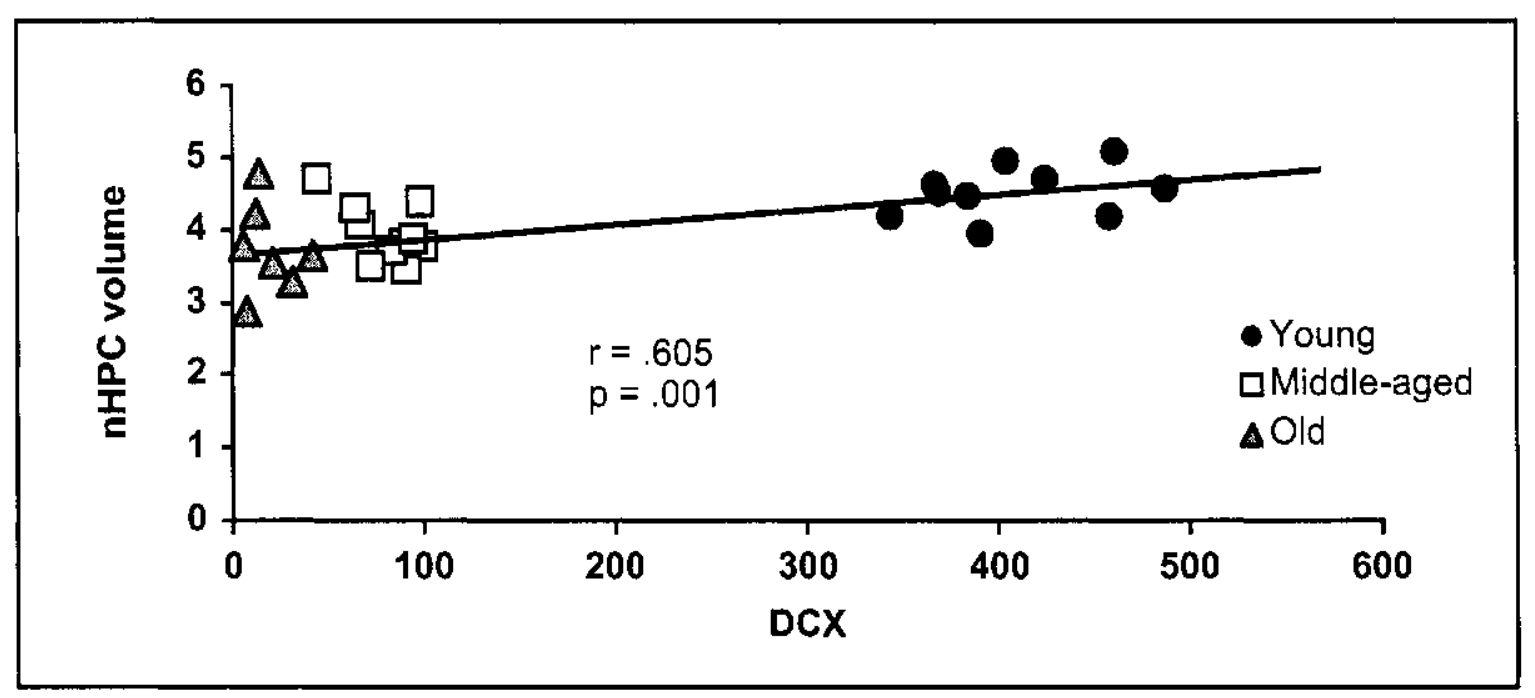

Figure 37. Correlations between the normalized hippocampal (nHPC) volume of young (black circles), middle-aged (open squares), and elderly (gray triangles) rats with the number of DCX-labeled cells. Higher number of DCX-labeled cells was correlated with larger hippocampal volume $(p=.001)$. 


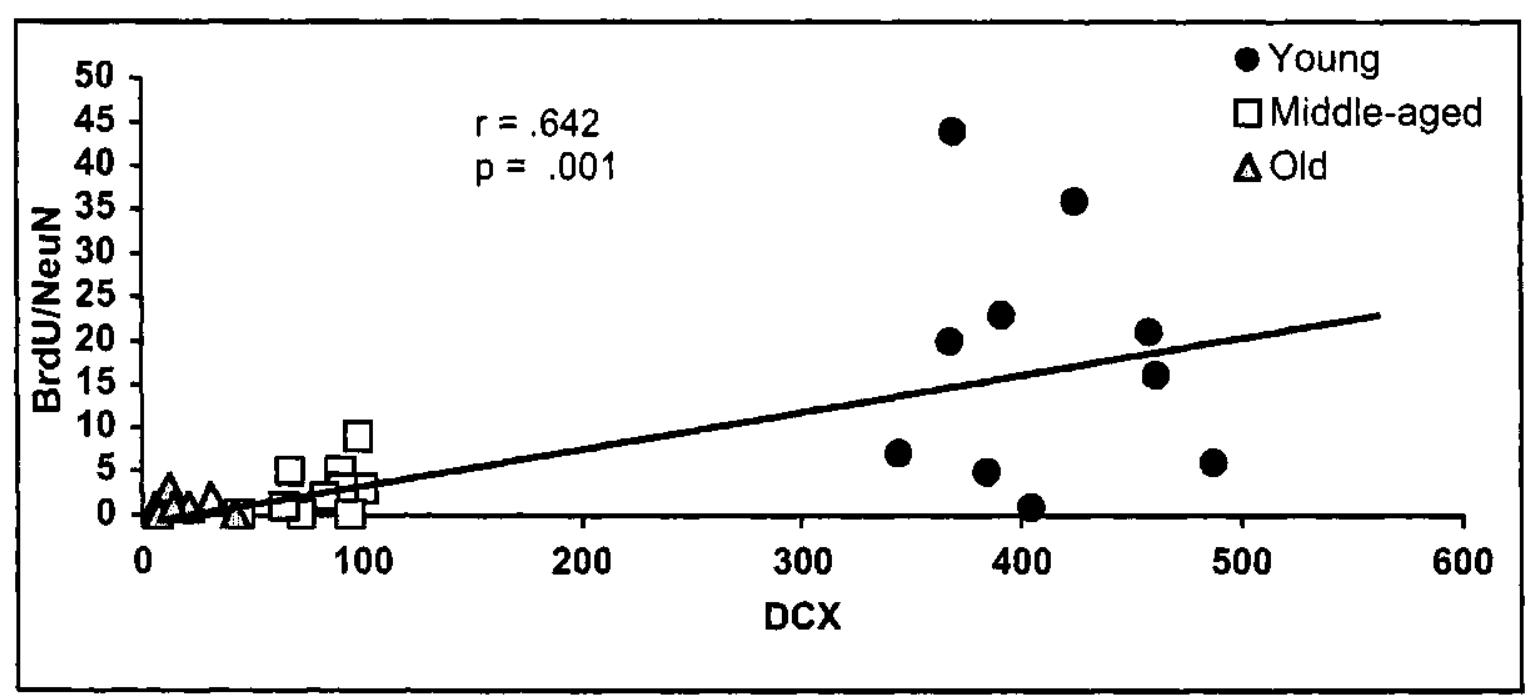

Figure 38. Correlations between the number of DCX-labeled cells in young (black circles), middle-aged (open squares), and elderly (gray triangles) rats and the number of BrdU/NeuN double-labeled cells. Higher number of DCXlabeled cells was correlated with a higher number of BrdU/NeuN double-labeled cells $(p=.001)$. 


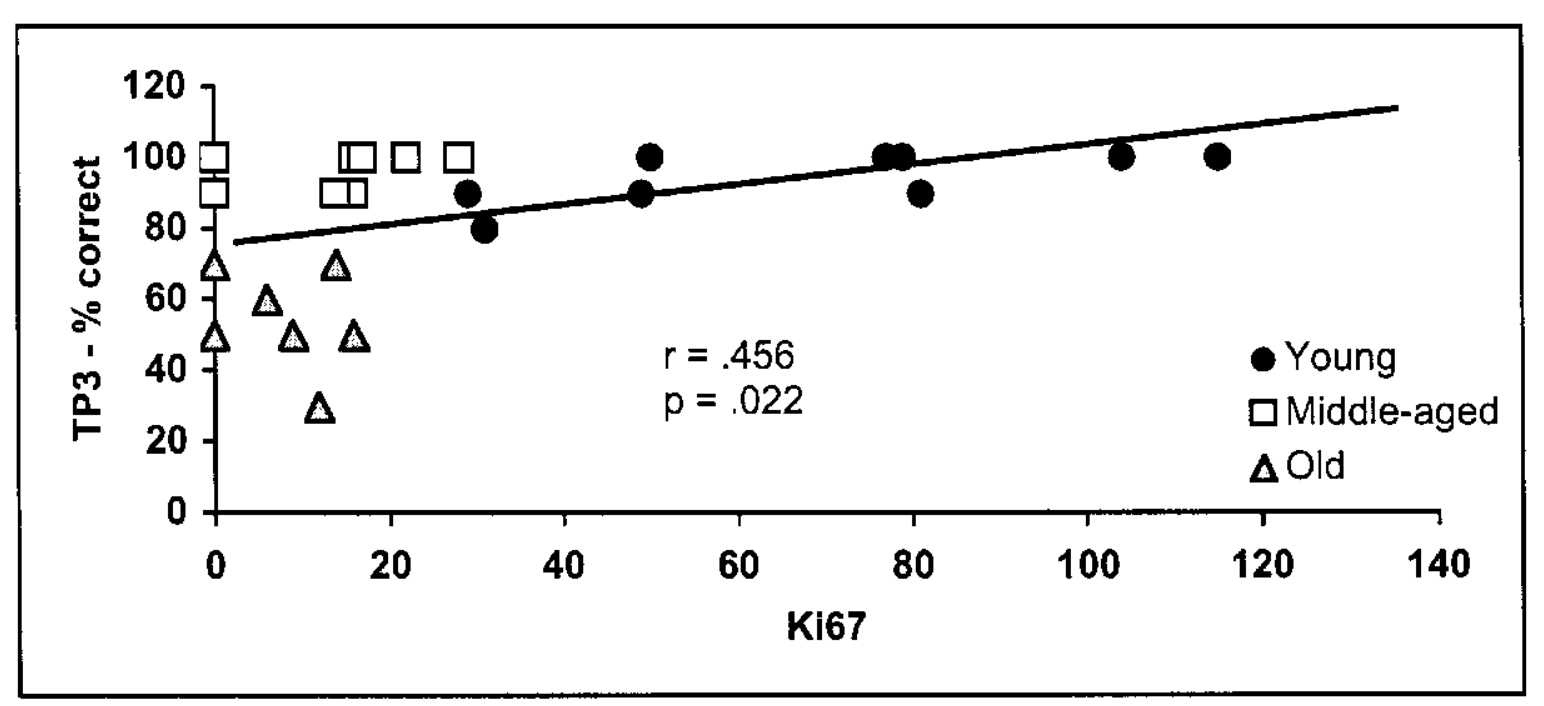

Figure 39. Correlations between performance of young (black circles), middleaged (open squares), and elderly (gray triangles) rats on the transverse patterning task with the number of Ki67-labeled cells. Better performance on Problem 3 of the transverse patterning task significantly correlated with a higher number of Ki67-labeled cells $(p=.022)$. 


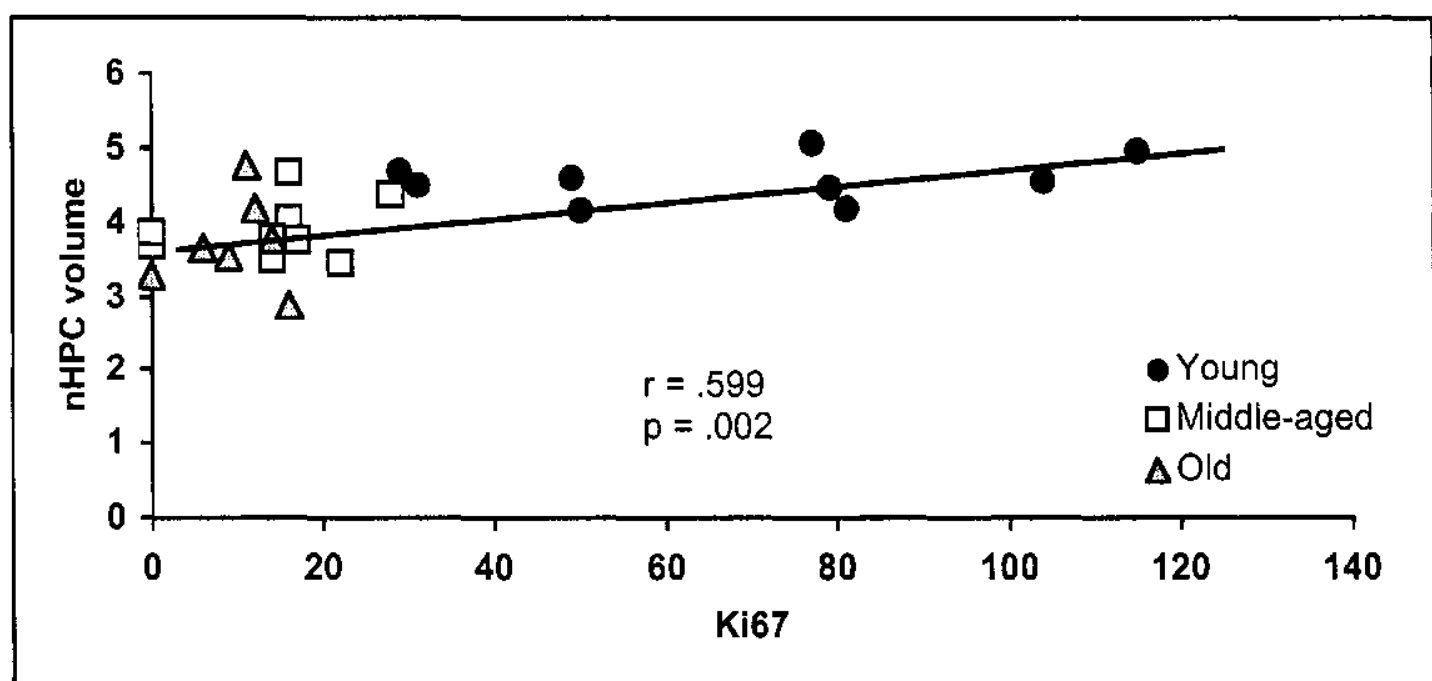

Figure 40. Correlations between the normalized hippocampal (nHPC) volume of young (black circles), middle-aged (open squares), and elderly (gray triangles) rats with the number of Ki67-labeled cells. Higher number of Ki67labeled cells was correlated with larger hippocampal volume $(p=.002)$. 


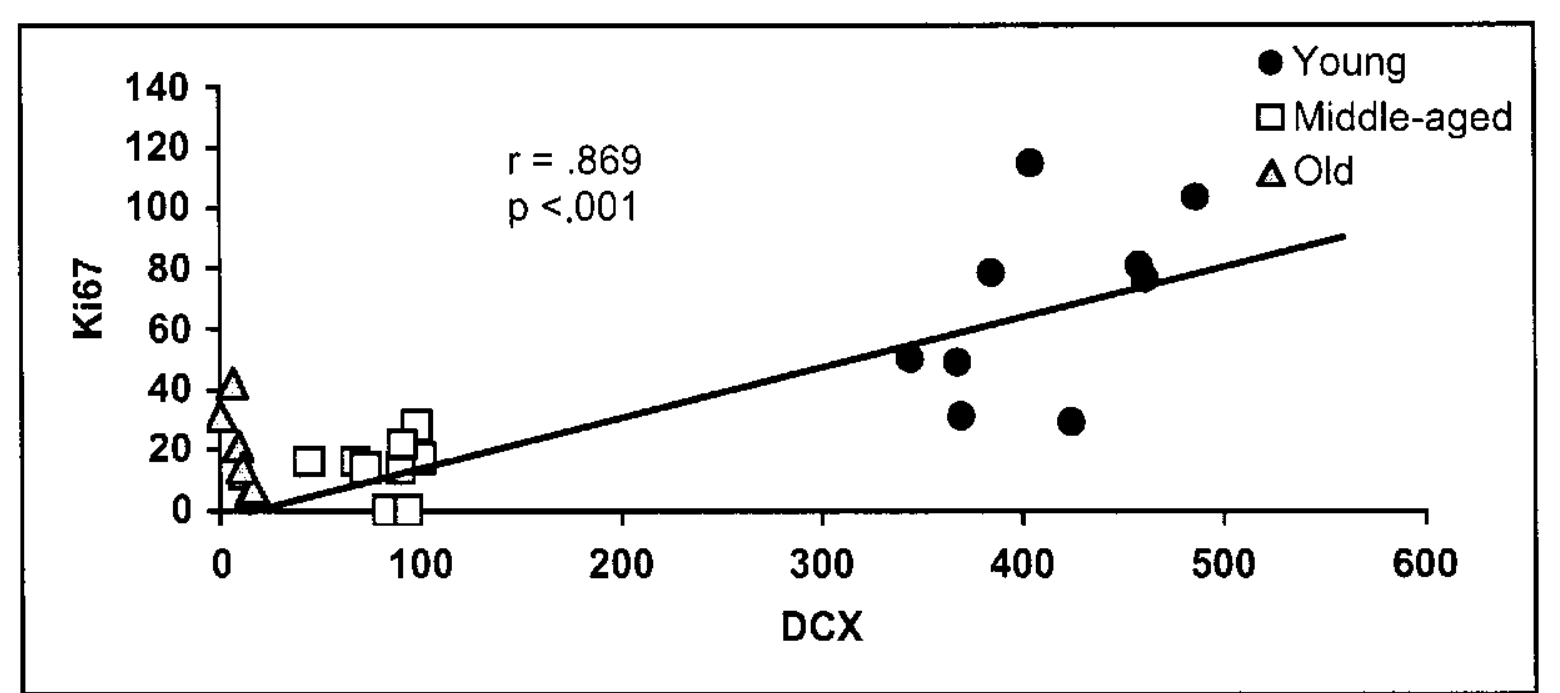

Figure 41. Correlations between the number of DCX-labeled cells in young (black circles), middle-aged (open squares), and elderly (gray triangles) rats and the number of Ki67-labeled cells. Rats with a higher number of DCX-labeled cells also had a higher number of Ki67-labeled cells $(p<.001)$. 